Dolabellane Diterpenes and Elemane Alkaloids from the Soft Coral Clavularia inflata

\title{
Collected in the South China Sea
}

Xiao Han, ${ }^{\dagger, \downarrow, \perp}$ Xiangchao Luo, ${ }^{\dagger, \downarrow, \perp}$ Lei Xue,,$^{\dagger,+, \perp}$ Leen van Ofwegen, ${ }^{\S}$ Wenjie

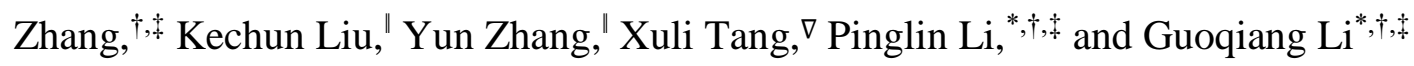

${ }^{t}$ Key Laboratory of Marine Drugs, Chinese Ministry of Education, School of Medicine and Pharmacy, Ocean University of China, Qingdao 266003, People's Republic of China

${ }^{\ddagger}$ Laboratory of Marine Drugs and Biological Products, Pilot National Laboratory for Marine

Science and Technology, Qingdao 266235, People's Republic of China

${ }^{\S}$ National Natuurhistorisch Museum, 2300 RA Leiden, The Netherlands

"Biology Institute, Qilu University of Technology (Shandong Academy of Sciences), Jinan 28789,

People's Republic of China

${ }^{\nabla}$ College of Chemistry and Chemical Engineering, Ocean University of China, Qingdao 266100, People's Republic of China

\section{Corresponding Author}

*E-mail: liguoqiang@ouc.edu.cn. Tel: +86-532-82032323. Fax: +86-551-8203-3663.

*E-mail: lipinglin@ouc.edu.cn. Tel: +86-532-82031535. Fax: +86-551-8203-3663. 


\section{Contents}

The detailed photographs of the soft coral Clavularia inflata

Figure S1. Detailed DP4+ probability (calculated at PCM/b3lyp/6-311+G(d,p) level) for compound 1. Isomer 1 is $(1 R, 8 R, 10 R, 11 S)-1 \mathbf{a}$, isomer 2 is $(1 R, 8 S, 10 R, 11 S)-\mathbf{1 b}$.

Table S1. Stable conformers of compound $\mathbf{1}$ with $(1 R, 8 R, 10 R, 11 S)-\mathbf{1 a}$ and $(1 R, 8 R, 10 R, 11 S)-\mathbf{1 b}$ configurations, respectively......

Table S2 . Important thermodynamic parameters (a.u.) of the optimized compound $\mathbf{1}$ with simplified structures at B3LYP/6-31+G(d,p) level in the gas phase

Table S3. Optimized Z-Matrixes of compound 1 with simplified structures in the Gas Phase ( $\AA$ ) at B3LYP/6-31+G(d,p) level.

Figure S2. Detailed DP4+ probability (calculated at PCM/b3lyp/6-311+G(d,p) level) for compound

2. Isomer 1 is $(1 R, 8 R, 10 R, 11 R)-\mathbf{2 a}$, isomer 2 is $(1 R, 8 R, 10 R, 11 S)-\mathbf{2 b}$, isomer 3 is $(1 R, 8 S, 10 R$, $11 R)-\mathbf{2 c}$, and isomer 4 is $(1 R, 8 S, 10 R, 11 S)$-2d.

Figure S3. Experimental and calculated ECD spectra of 2

Table S4. Stable conformers of compound $\mathbf{2}$ with $(1 R, 8 R, 10 R, 11 R)-\mathbf{2 a},(1 R, 8 R, 10 R, 11 S)-\mathbf{2 b}$, $(1 R, 8 S, 10 R, 11 R)-\mathbf{2 c}$ and $(1 R, 8 S, 10 R, 11 S)$-2d configurations, respectively.

Table S5. Important thermodynamic parameters (a.u.) of the optimized compound 2 with simplified structures at $\mathrm{B} 3 \mathrm{LYP} / 6-31+\mathrm{G}(\mathrm{d}, \mathrm{p})$ level in the gas phase.

Table S6. Optimized Z-Matrixes of compound 2 with simplified structures in the Gas Phase $(\AA)$ at B3LYP/6-31+G(d,p) level.

Figure S4. Detailed DP4+ probability (calculated at PCM/b3lyp/6-311+G(d,p) level) for compound 3. Isomer 1 is $(1 S, 4 S, 6 S, 8 S, 10 R, 11 S)-\mathbf{3 a}$, isomer 2 is $(1 S, 4 S, 6 S, 8 R, 10 R, 11 S)-3 \mathbf{b}$, isomer 3 is $(1 S, 4 R, 6 R, 8 S, 10 R, 11 S)-\mathbf{3 c}$, and isomer 4 is $(1 S, 4 R, 6 R, 8 R, 10 R, 11 S)-\mathbf{3 d}$.

Table S7. Stable conformers of compound 3 with $(1 S, 4 S, 6 S, 8 S, 10 R, 11 S)-3 \mathbf{a},(1 S, 4 S, 6 S, 8 R$, $10 R, 11 S)-\mathbf{3 b},(1 S, 4 R, 6 R, 8 S, 10 R, 11 S)-\mathbf{3 c}$ and $(1 S, 4 R, 6 R, 8 R, 10 R, 11 S)-\mathbf{3 d}$ configurations, respectively.

Table S8. Important thermodynamic parameters (a.u.) of the optimized compound $\mathbf{3}$ with simplified structures at B3LYP/6-31+G(d,p) level in the gas phase.

Table S9. Optimized Z-Matrixes of compound 3 with simplified structures in the Gas Phase $(\AA)$ at B3LYP/6-31+G(d,p) level.

Figure S5. Detailed DP4+ probability (calculated at $\mathrm{PCM} / \mathrm{b} 31 \mathrm{yp} / 6-311+\mathrm{G}(\mathrm{d}, \mathrm{p})$ level) for compound 4. Isomer 1 is $(1 R, 8 R, 10 R, 11 S)-\mathbf{4 a}$, isomer 2 is $(1 R, 8 S, 10 R, 11 S)-\mathbf{4 b}$.

Figure S6. Experimental and calculated ECD spectra of 4

Table S10. Stable conformers of compound 4 with $(1 R, 8 R, 10 R, 11 S)$-4a and $(1 R, 8 S, 10 R, 11 S)$ 4b configurations, respectively.

Table S11. Important thermodynamic parameters (a.u.) of the optimized compound $\mathbf{4}$ with simplified structures at B3LYP/6-31+G(d,p) level in the gas phase.

Table S12. Optimized Z-Matrixes of compound 4 with simplified structures in the Gas Phase $(\AA)$ at B3LYP/6-31+G(d,p) level.

Figure S7. Detailed DP4+ probability (calculated at $\mathrm{PCM} / \mathrm{b} 31 \mathrm{yp} / 6-311+\mathrm{G}(\mathrm{d}, \mathrm{p})$ level) for compound 5. Isomer 1 is $(1 R, 10 R, 11 S)-\mathbf{5 a}$, isomer 2 is $(1 R, 10 S, 11 S)-\mathbf{5 b}$

Table S13. Stable conformers of compound $\mathbf{3}$ with $(1 R, 10 R, 11 S)-\mathbf{5 a}$ and $(1 R, 10 S, 11 S)-\mathbf{5 b}$ 
configurations, respectively.......

Table S14. Important thermodynamic parameters (a.u.) of the optimized compound $\mathbf{5}$ with simplified structures at B3LYP/6-31+G(d,p) level in the gas phase.

.26

Table S15. Optimized Z-Matrixes of compound 5 with simplified structures in the Gas Phase ( $\AA$ ) at

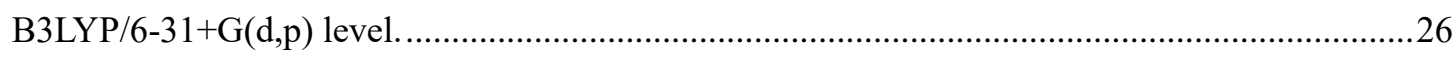

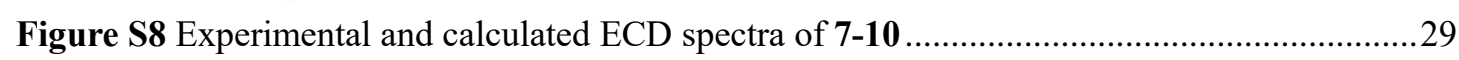

Table S16. Stable conformers of compound 7 with $(1 S, 6 R, 9 S)-7 \mathbf{a}$ and $(1 R, 6 S, 9 R)-7 \mathbf{b}$ configurations, respectively.

Table S17. Important thermodynamic parameters (a.u.) of the optimized compound 7 with structures at B3LYP/6-31+G(d,p) level in the gas phase.

Table S18. Optimized Z-Matrixes of compound 7 with structures in the Gas Phase $(\AA)$ at B3LYP/6$31+\mathrm{G}(\mathrm{d}, \mathrm{p})$ level.

Table S19. Stable conformers of compound $\mathbf{8}$ with $(1 R, 6 R)-\mathbf{8 a}$ and $(1 S, 6 S)-\mathbf{8 b}$ configurations, respectively.

Table S20. Important thermodynamic parameters (a.u.) of the optimized compound $\mathbf{8}$ with structures at B3LYP/6-31+G(d,p) level in the gas phase.

Table S21. Optimized Z-Matrixes of compound 8 with structures in the Gas Phase $(\AA)$ at B3LYP/6-

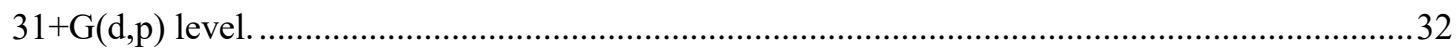

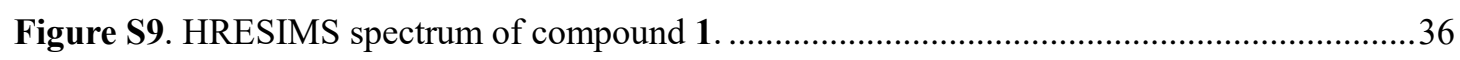

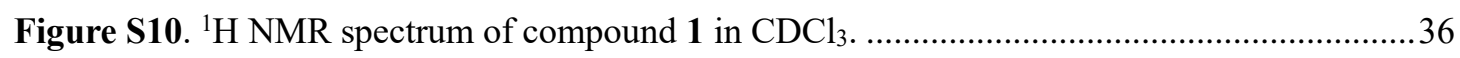

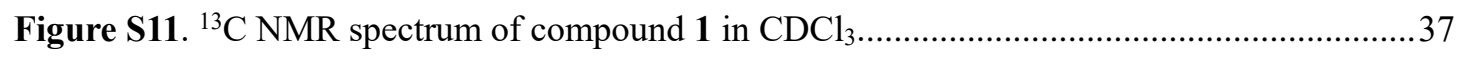

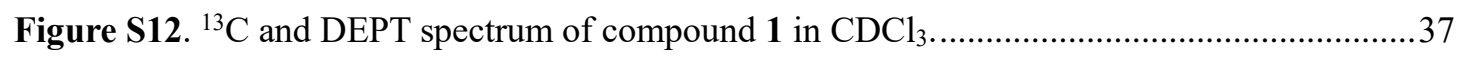

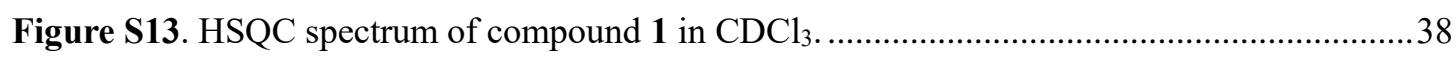

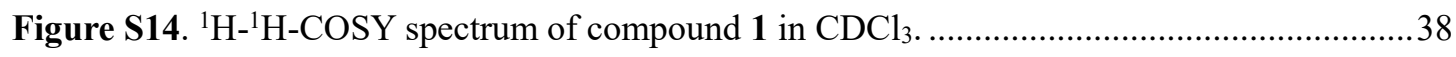

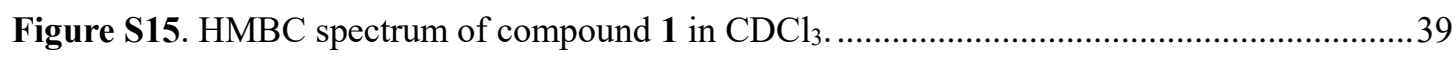

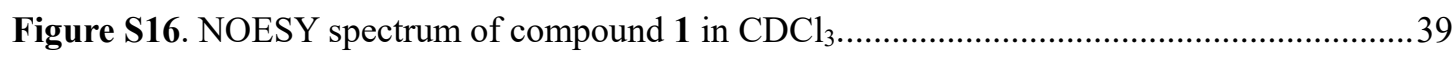

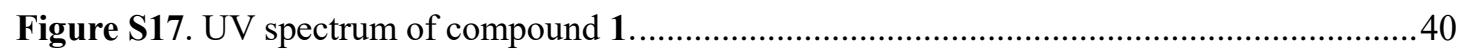

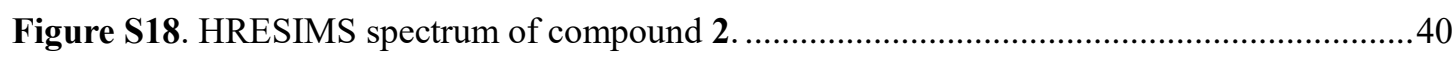

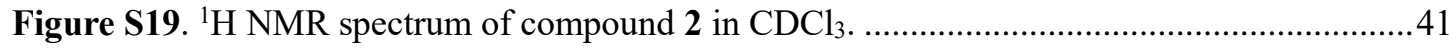

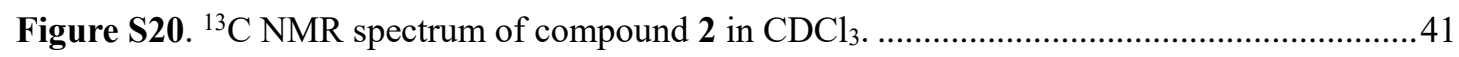

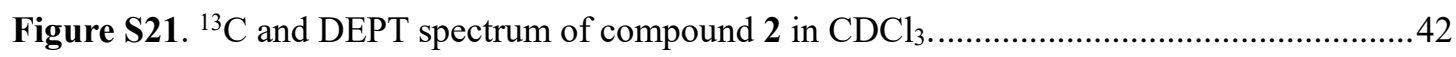

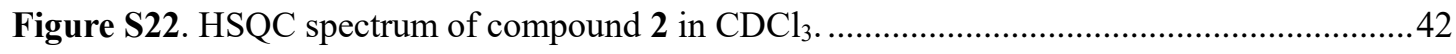

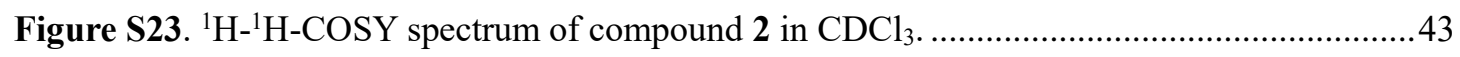

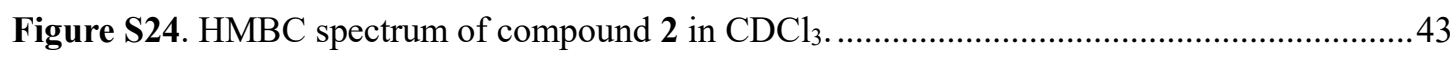

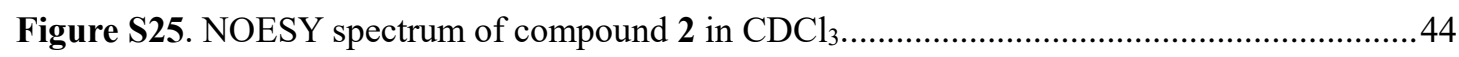

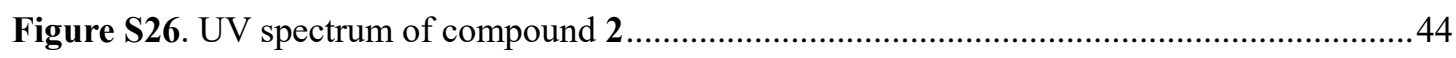

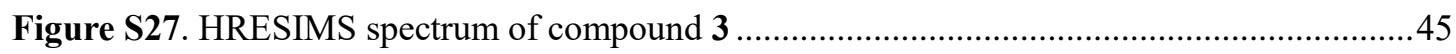

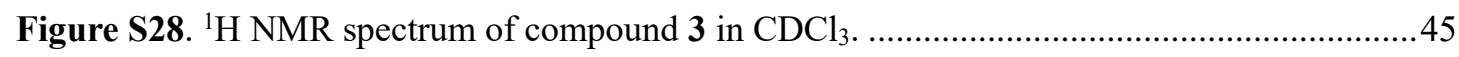

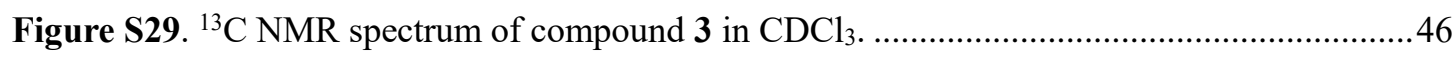

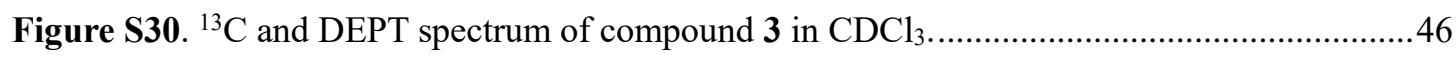

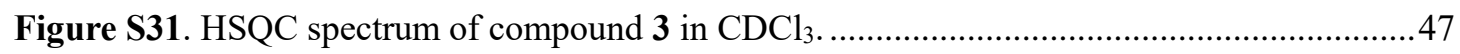

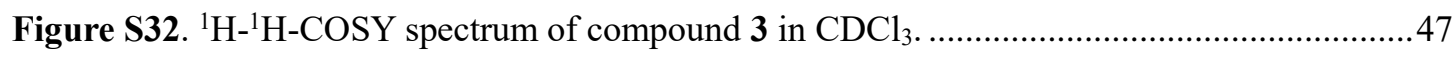

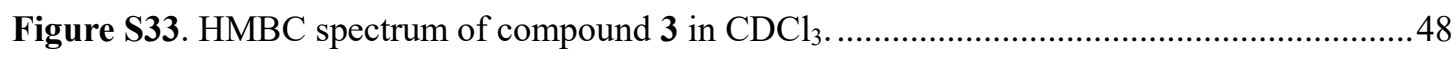

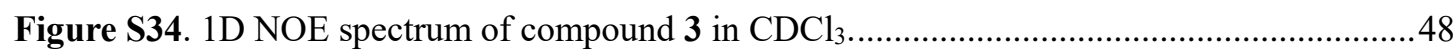


Figure S35. UV spectrum of compound 3.

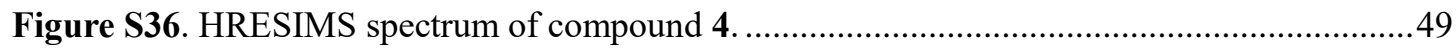

Figure S37. ${ }^{1} \mathrm{H}$ NMR spectrum of compound 4 in $\mathrm{CDCl}_{3}$. .................................................50

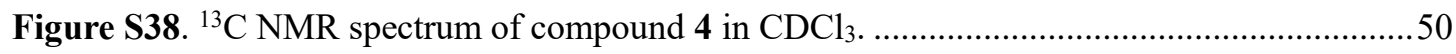

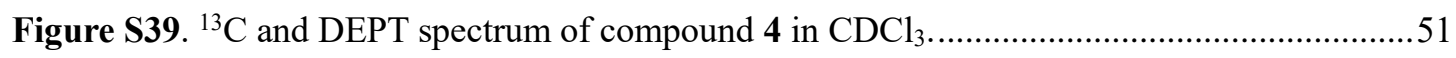

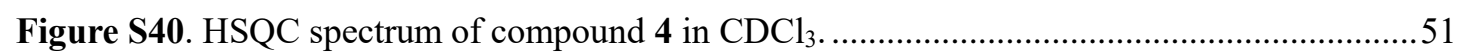

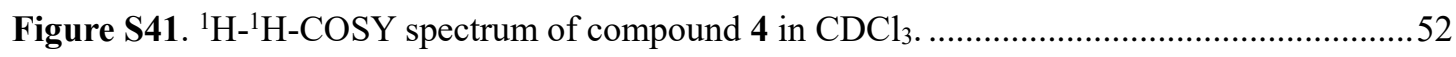

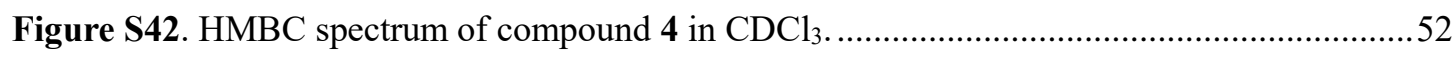

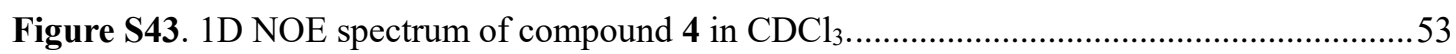

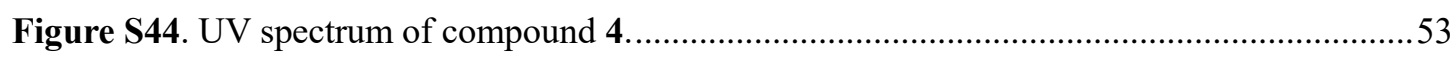

Figure S45. HRESIMS spectrum of compound 5 ..........................................................5

Figure S46. ${ }^{1} \mathrm{H}$ NMR spectrum of compound $\mathbf{5}$ in $\mathrm{CDCl}_{3}$.................................................5

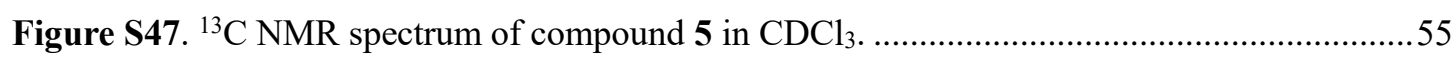

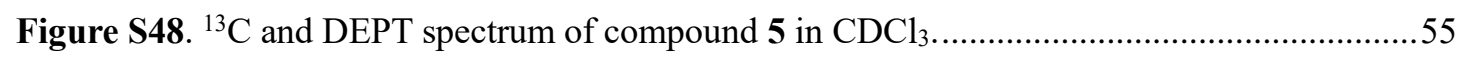

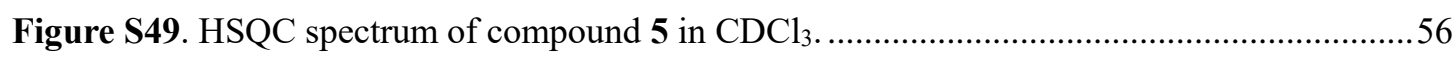

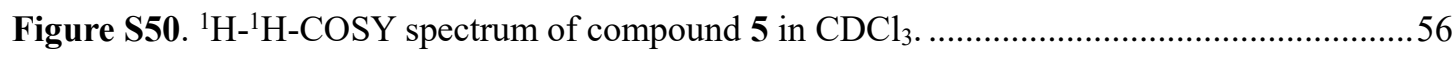

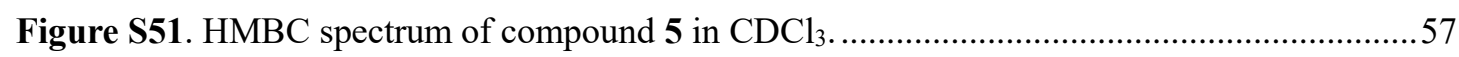

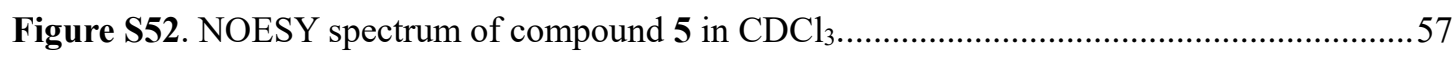

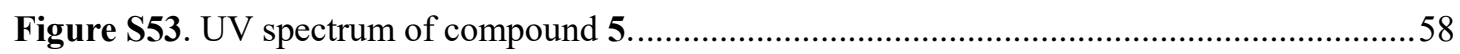

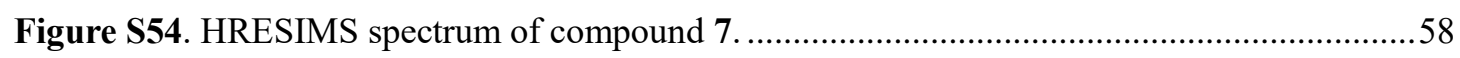

Figure S55. ${ }^{1} \mathrm{H}$ NMR spectrum of compound 7 in $\mathrm{CDCl}_{3}$..................................................59

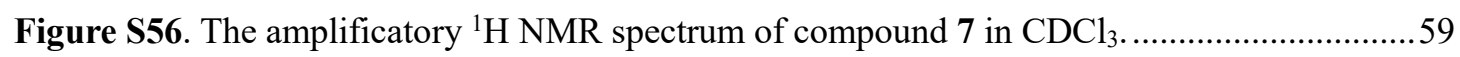

Figure S57. ${ }^{13} \mathrm{C}$ NMR spectrum of compound 7 in $\mathrm{CDCl}_{3}$...................................................60

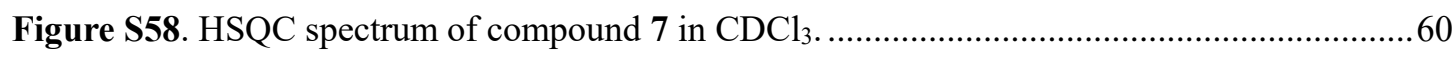

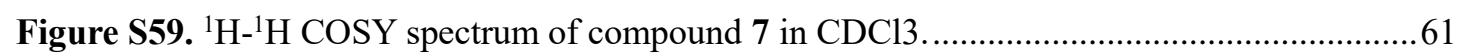

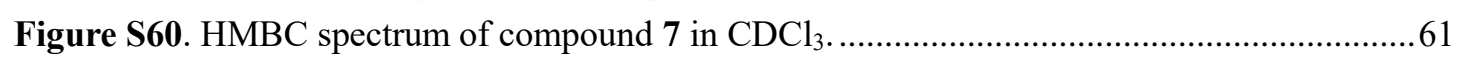

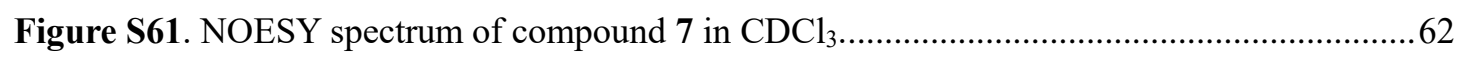

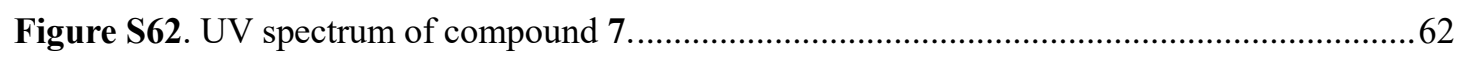

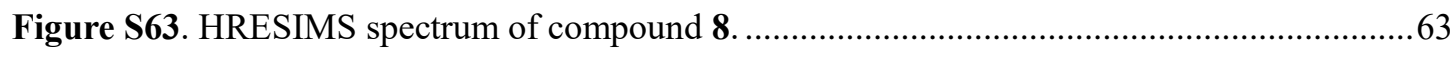

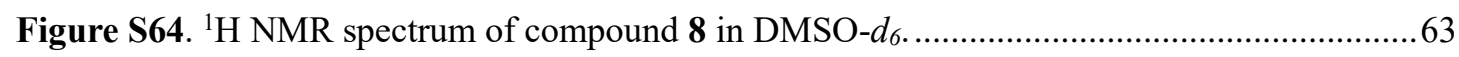

Figure S65. The amplificatory ${ }^{1} \mathrm{H}$ NMR spectrum of compound $\mathbf{8}$ in DMSO- $d_{6} . \ldots \ldots \ldots \ldots \ldots \ldots \ldots . . . . . . . . . . .64$

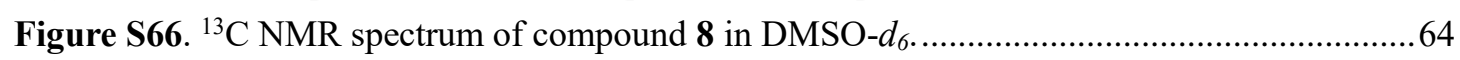

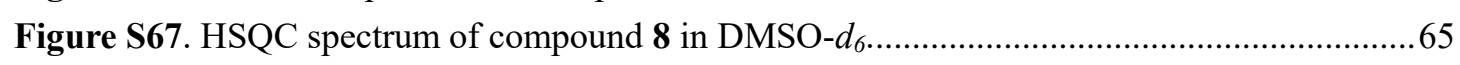

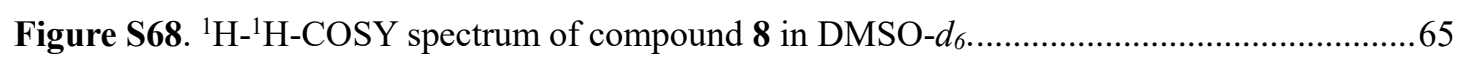

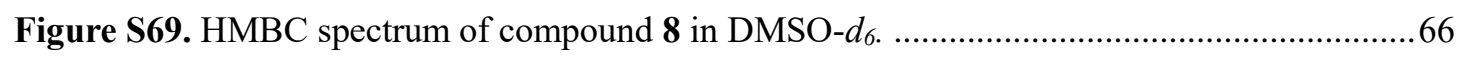

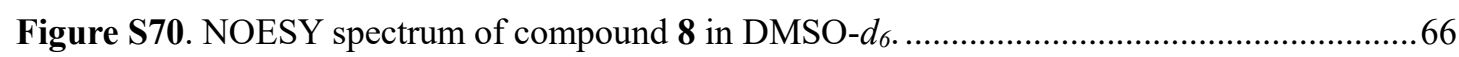

Figure S71. UV spectrum of compound 8....................................................................67

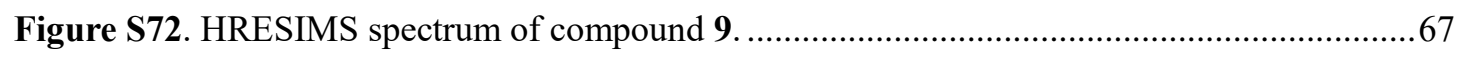

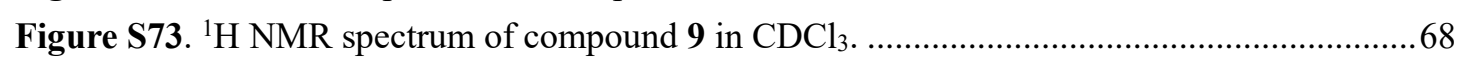

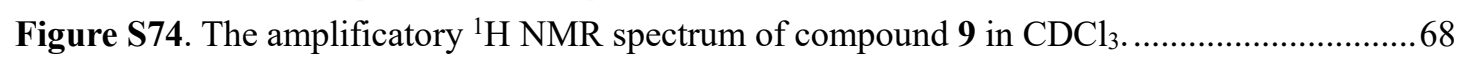

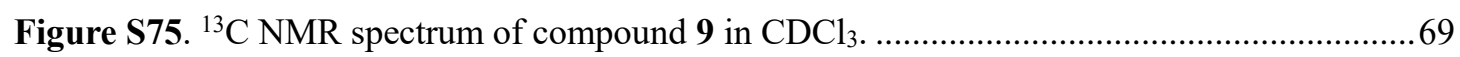

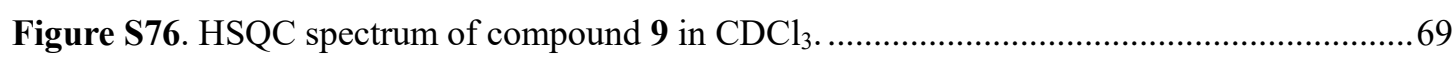

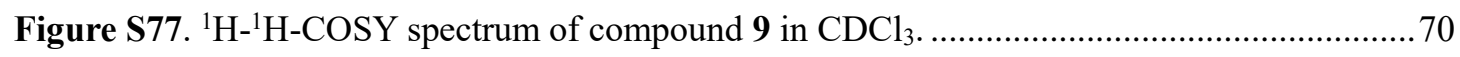

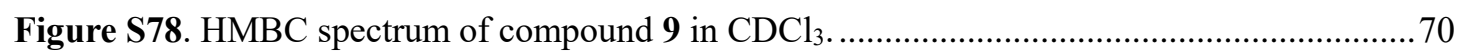




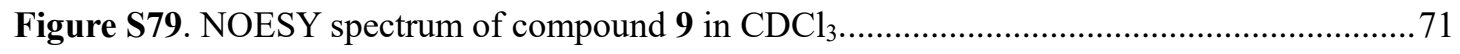

Figure S80. UV spectrum of compound 9..................................................................... 71

Figure S81. HRESIMS spectrum of compound 10 ......................................................... 72

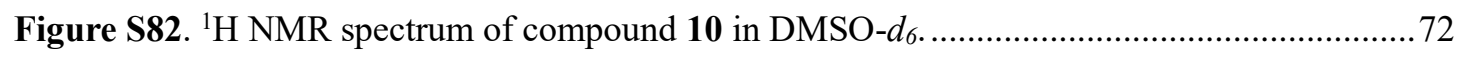

Figure S83. The amplificatory ${ }^{1} \mathrm{H}$ NMR spectrum of compound $\mathbf{1 0}$ in DMSO- $d_{6} . \ldots \ldots \ldots \ldots \ldots \ldots . . . . . . . . . . . .73$

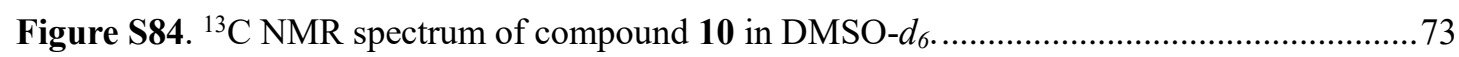

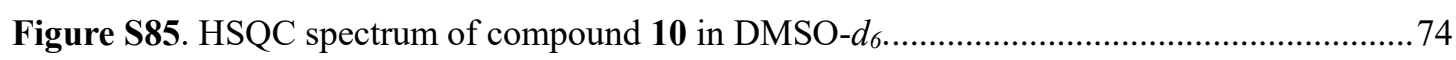

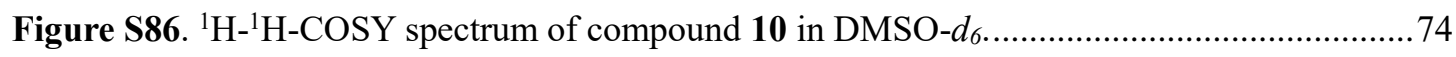

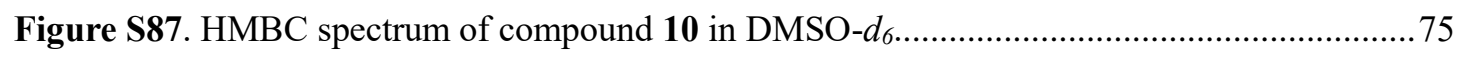

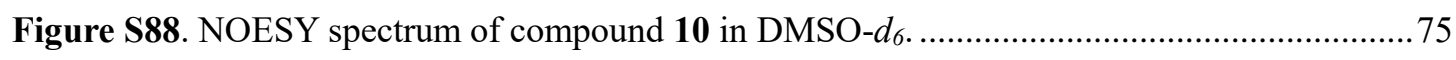

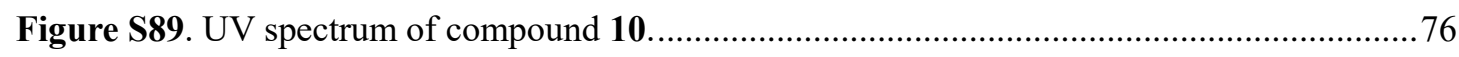

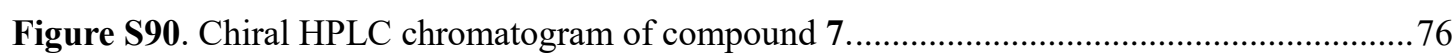


The detailed photographs of the soft coral Clavularia inflata
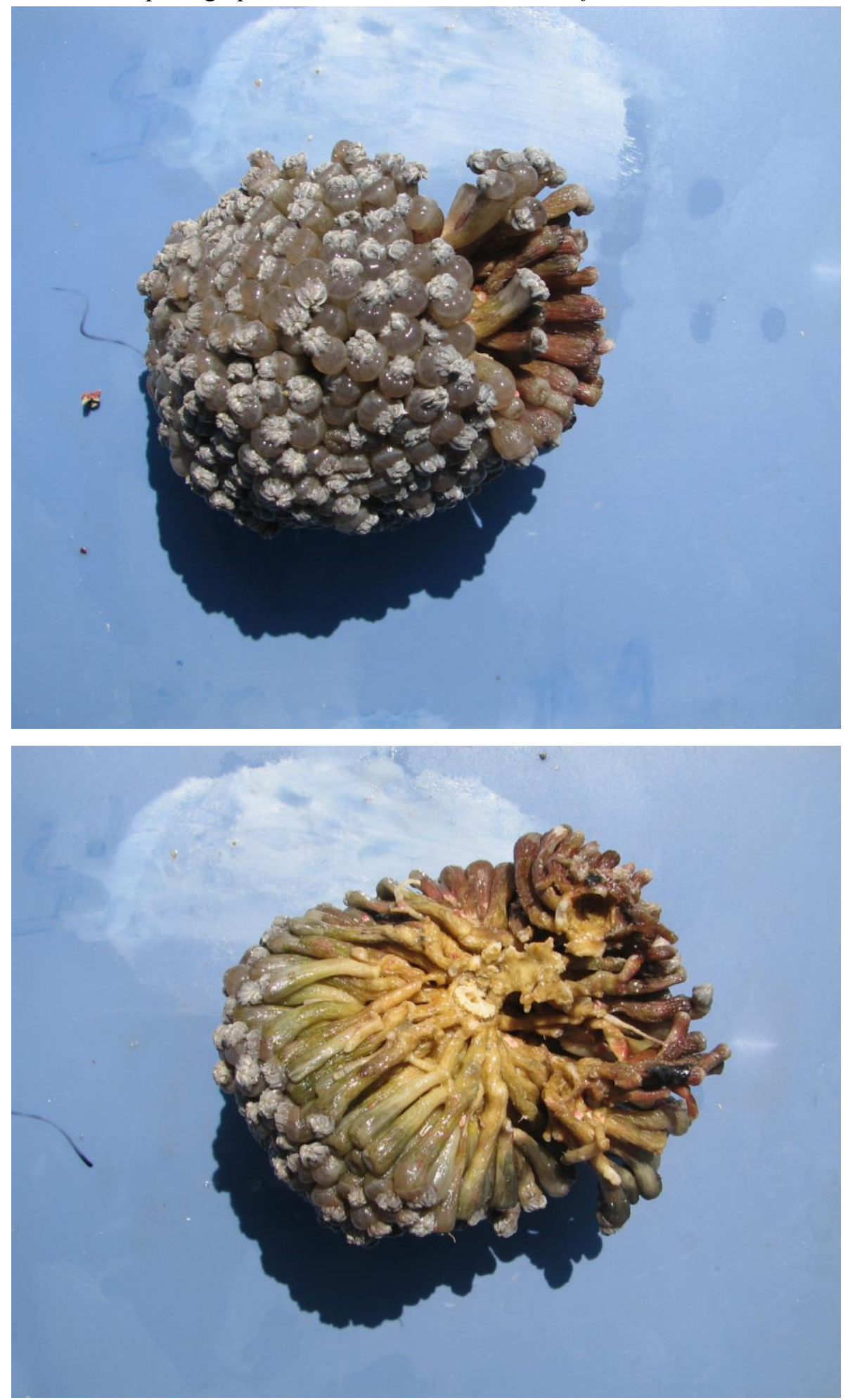


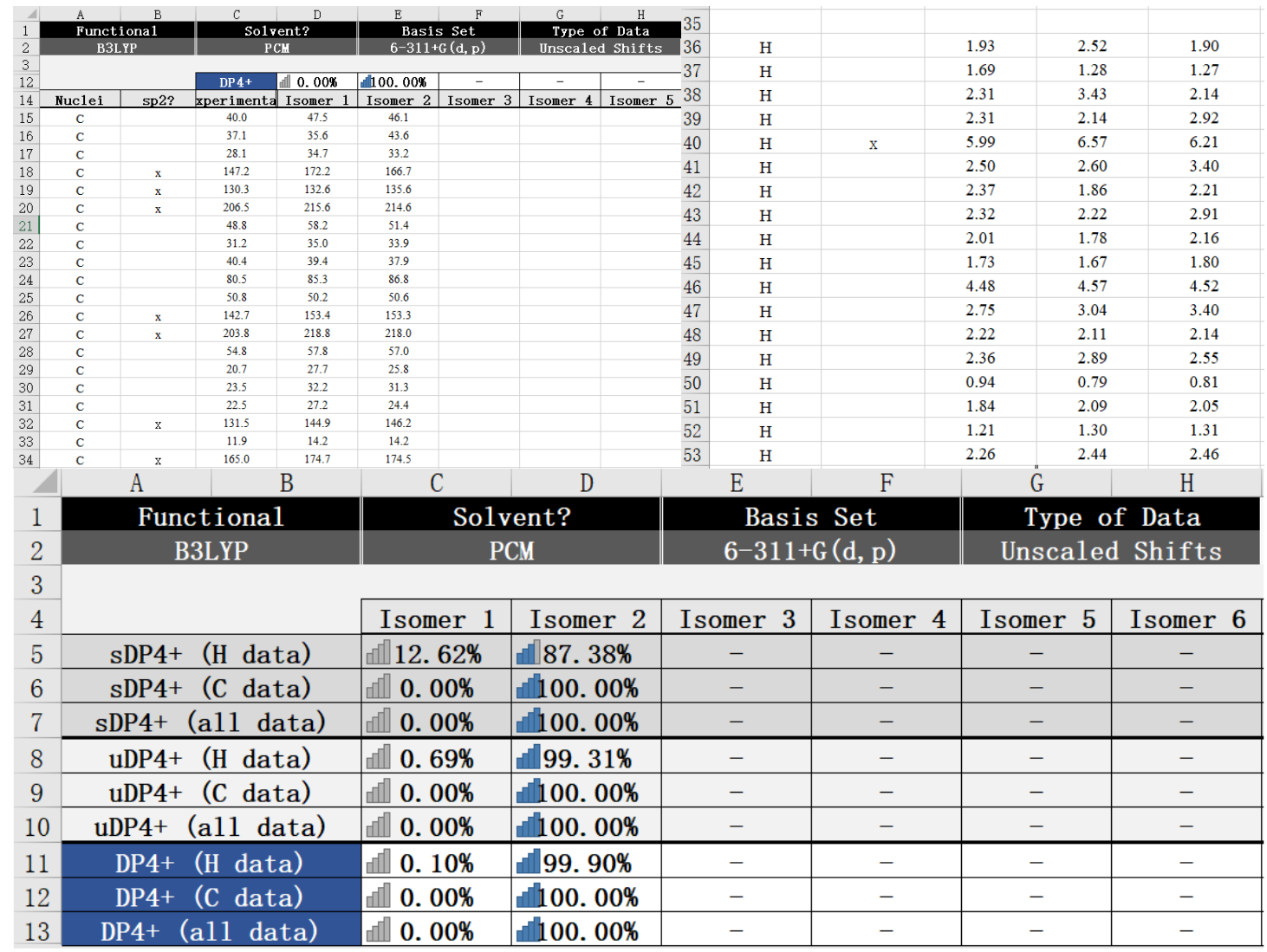

Figure S1. Detailed DP4+ probability (calculated at PCM/b3lyp/6-311+G(d,p) level) for compound 1. Isomer 1 is $(1 R, 8 R, 10 R, 11 S)$-1a, isomer 2 is $(1 R, 8 S, 10 R, 11 S)$-1 $\mathbf{b}$.

Table S1. Stable conformers of compound $\mathbf{1}$ with $(1 R, 8 R, 10 R, 11 S)-\mathbf{1 a}$ and $(1 R, 8 R, 10 R, 11 S)-\mathbf{1 b}$ configurations, respectively.

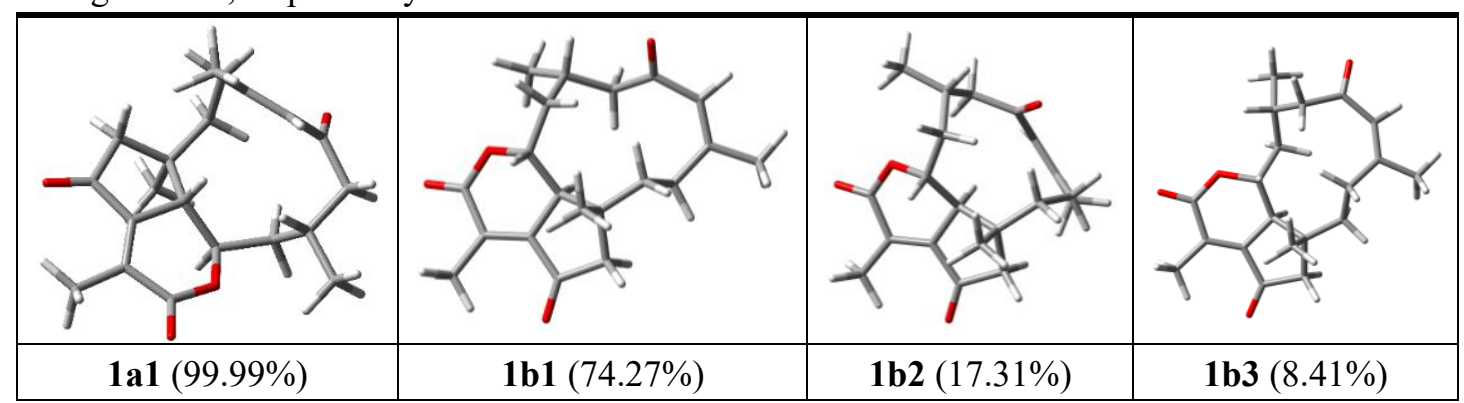

Table S2. Important thermodynamic parameters (a.u.) of the optimized compound 1 with simplified structures at B3LYP/6-31+G(d,p) level in the gas phase.

\begin{tabular}{|c|c|c|}
\hline NO. & E+ZPE & G \\
\hline $\mathbf{1 a 1}$ & -1078.276173 & -1078.326458 \\
\hline $\mathbf{1 b 1}$ & -1078.270576 & -1078.321246 \\
\hline $\mathbf{1 b 2}$ & -1078.269808 & -1078.319872 \\
\hline $\mathbf{1 b 3}$ & -1078.267860 & -1078.319191 \\
\hline
\end{tabular}

Table S3. Optimized Z-Matrixes of compound 1 with simplified structures in the Gas Phase $(\AA)$ at B3LYP/6-31+G(d,p) level. 


\begin{tabular}{|c|c|c|c|c|c|c|c|c|c|c|c|}
\hline \multicolumn{4}{|c|}{$1 \mathrm{a} 1$} & \multicolumn{4}{|c|}{$1 b 1$} & \multicolumn{4}{|c|}{$1 b 2$} \\
\hline $\mathrm{C}$ & 2.434044 & -1.83061 & 0.10961 & $\mathrm{C}$ & -2.15631 & -2.30945 & -0.25484 & $\mathrm{C}$ & -2.10503 & -2.05268 & 0.064144 \\
\hline $\mathrm{C}$ & 2.07821 & -0.37734 & 0.122149 & $\mathrm{C}$ & -2.20155 & -0.81505 & -0.20521 & $\mathrm{C}$ & -1.89362 & -0.58054 & -0.08936 \\
\hline $\mathrm{C}$ & 0.613897 & -0.22006 & -0.25622 & $\mathrm{C}$ & -0.80645 & -0.2873 & 0.085965 & $\mathrm{C}$ & -0.47784 & -0.2286 & 0.337879 \\
\hline $\mathrm{C}$ & 0.295325 & -1.55295 & -1.05791 & $\mathrm{C}$ & -0.08747 & -1.51135 & 0.781951 & $\mathrm{C}$ & -0.03176 & -1.45672 & 1.236554 \\
\hline $\mathrm{C}$ & 1.18758 & -2.58592 & -0.32507 & $\mathrm{C}$ & -0.7171 & -2.71553 & 0.036741 & $\mathrm{C}$ & -0.79641 & -2.63366 & 0.579105 \\
\hline $\mathrm{C}$ & 0.432288 & 1.136086 & -0.96083 & $\mathrm{C}$ & -0.93709 & 1.036485 & 0.855387 & $\mathrm{C}$ & -0.52762 & 1.187397 & 0.943923 \\
\hline $\mathrm{C}$ & -0.99496 & 1.648815 & -1.21083 & $\mathrm{C}$ & 0.291855 & 1.904725 & 1.180445 & $\mathrm{C}$ & 0.76071 & 1.878099 & 1.436287 \\
\hline $\mathrm{C}$ & -1.18953 & -1.99485 & -1.12148 & $\mathrm{C}$ & 1.454086 & -1.48184 & 0.689791 & $\mathrm{C}$ & 1.498316 & -1.71129 & 1.326164 \\
\hline $\mathrm{C}$ & -1.74233 & 2.193146 & 0.041481 & $\mathrm{C}$ & 1.141161 & 2.610123 & 0.0866 & $\mathrm{C}$ & 1.674992 & 2.681853 & 0.466216 \\
\hline $\mathrm{C}$ & -3.22679 & 1.741884 & 0.071766 & $\mathrm{C}$ & 2.080942 & 1.688763 & -0.71192 & $\mathrm{C}$ & 2.057023 & 1.945983 & -0.83086 \\
\hline $\mathrm{C}$ & -3.34281 & 0.227669 & 0.119632 & $\mathrm{C}$ & 3.298654 & 1.185045 & 0.062599 & $\mathrm{C}$ & 2.753114 & 0.613275 & -0.57706 \\
\hline $\mathrm{C}$ & -2.74622 & -0.41947 & 1.31396 & $\mathrm{C}$ & 4.03807 & -0.02881 & -0.39456 & $\mathrm{C}$ & 2.478651 & -0.46881 & -1.55225 \\
\hline $\mathrm{C}$ & 2.902315 & 0.662969 & 0.36409 & $\mathrm{C}$ & -3.28214 & -0.01839 & -0.33384 & $\mathrm{C}$ & -2.79113 & 0.342064 & -0.49526 \\
\hline $\mathrm{O}$ & 1.069998 & 2.187859 & -0.17629 & $\mathrm{O}$ & -1.85993 & 1.913444 & 0.144448 & $\mathrm{O}$ & -1.13566 & 2.080533 & -0.03557 \\
\hline $\mathrm{C}$ & 2.308957 & 2.036931 & 0.352469 & $\mathrm{C}$ & -3.06025 & 1.463253 & -0.29647 & $\mathrm{C}$ & -2.31149 & 1.754846 & -0.63106 \\
\hline $\mathrm{C}$ & 0.795952 & -1.43127 & -2.51545 & $\mathrm{C}$ & -0.47528 & -1.60405 & 2.274033 & $\mathrm{C}$ & -0.56183 & -1.28306 & 2.678385 \\
\hline $\mathrm{O}$ & 2.871274 & 2.998183 & 0.829618 & $\mathrm{O}$ & -3.88872 & 2.256248 & -0.68663 & $\mathrm{O}$ & -2.89987 & 2.589638 & -1.28257 \\
\hline $\mathrm{O}$ & -3.8321 & -0.40609 & -0.80916 & $\mathrm{O}$ & 3.756503 & 1.838993 & 0.990294 & $\mathrm{O}$ & 3.471207 & 0.450633 & 0.402594 \\
\hline $\mathrm{C}$ & -1.64928 & 3.724003 & 0.130428 & $\mathrm{C}$ & 0.337517 & 3.515133 & -0.86155 & $\mathrm{C}$ & 1.134604 & 4.086533 & 0.152099 \\
\hline $\mathrm{C}$ & 4.358682 & 0.591048 & 0.714053 & $\mathrm{C}$ & -4.69287 & -0.45762 & -0.58669 & $\mathrm{C}$ & -4.21047 & 0.092832 & -0.90901 \\
\hline $\mathrm{C}$ & -1.92559 & -2.52242 & 0.136864 & $\mathrm{C}$ & 2.088579 & -1.62997 & -0.71161 & $\mathrm{C}$ & 2.269621 & -2.36778 & 0.150624 \\
\hline $\mathrm{C}$ & -2.11389 & -1.61106 & 1.340821 & $\mathrm{C}$ & 3.561481 & -1.24323 & -0.73112 & $\mathrm{C}$ & 2.259546 & -1.76382 & -1.24945 \\
\hline $\mathrm{C}$ & -1.5586 & -2.1322 & 2.642351 & $\mathrm{C}$ & 4.512838 & -2.35832 & -1.08383 & $\mathrm{C}$ & 1.998306 & -2.75315 & -2.35843 \\
\hline $\mathrm{O}$ & 3.505648 & -2.33624 & 0.391554 & $\mathrm{H}$ & -0.30625 & -0.09258 & -0.87287 & $\mathrm{H}$ & 0.158934 & -0.21497 & -0.55304 \\
\hline $\mathrm{H}$ & 0.00908 & -0.22272 & 0.660397 & $\mathrm{O}$ & -3.07511 & -3.07463 & -0.48415 & $\mathrm{O}$ & -3.11671 & -2.68757 & -0.17505 \\
\hline $\mathrm{H}$ & 1.443293 & -3.46277 & -0.92643 & $\mathrm{H}$ & -0.23174 & -2.88333 & -0.93406 & $\mathrm{H}$ & -0.97157 & -3.48297 & 1.245259 \\
\hline $\mathrm{H}$ & 0.702377 & -2.95414 & 0.588323 & $\mathrm{H}$ & -0.67264 & -3.65829 & 0.588674 & $\mathrm{H}$ & -0.25673 & -3.01664 & -0.29644 \\
\hline $\mathrm{H}$ & 0.96248 & 1.096474 & -1.91962 & $\mathrm{H}$ & -1.41382 & 0.804508 & 1.816724 & $\mathrm{H}$ & -1.21719 & 1.143415 & 1.796219 \\
\hline $\mathrm{H}$ & -0.92783 & 2.452857 & -1.95276 & $\mathrm{H}$ & 0.960075 & 1.314913 & 1.818242 & $\mathrm{H}$ & 0.460567 & 2.579942 & 2.222981 \\
\hline $\mathrm{H}$ & -1.5639 & 0.848959 & -1.6931 & $\mathrm{H}$ & -0.09738 & 2.700029 & 1.827412 & $\mathrm{H}$ & 1.368162 & 1.11867 & 1.934504 \\
\hline $\mathrm{H}$ & -1.78726 & -1.20456 & -1.57572 & $\mathrm{H}$ & 1.848456 & -2.28652 & 1.32352 & $\mathrm{H}$ & 2.002874 & -0.78915 & 1.611787 \\
\hline $\mathrm{H}$ & -1.22864 & -2.82437 & -1.83966 & $\mathrm{H}$ & 1.808961 & -0.55774 & 1.15227 & $\mathrm{H}$ & 1.649548 & -2.39546 & 2.171038 \\
\hline $\mathrm{H}$ & -1.25827 & 1.784708 & 0.936529 & $\mathrm{H}$ & 1.809868 & 3.258853 & 0.664411 & $\mathrm{H}$ & 2.60935 & 2.806959 & 1.026521 \\
\hline $\mathrm{H}$ & -3.70509 & 2.176335 & 0.959748 & $\mathrm{H}$ & 2.520361 & 2.274284 & -1.53465 & $\mathrm{H}$ & 1.182716 & 1.803383 & -1.4751 \\
\hline $\mathrm{H}$ & -3.76386 & 2.098504 & -0.81269 & $\mathrm{H}$ & 1.549889 & 0.876337 & -1.20785 & $\mathrm{H}$ & 2.753868 & 2.576998 & -1.4027 \\
\hline $\mathrm{H}$ & -2.77406 & 0.168223 & 2.230439 & $\mathrm{H}$ & 5.116789 & 0.09743 & -0.32134 & $\mathrm{H}$ & 2.370238 & -0.15182 & -2.58907 \\
\hline $\mathrm{H}$ & 0.179398 & -0.74108 & -3.09975 & $\mathrm{H}$ & -0.04229 & -0.78767 & 2.860299 & $\mathrm{H}$ & -0.04244 & -0.47885 & 3.208839 \\
\hline $\mathrm{H}$ & 1.833209 & -1.08333 & -2.56696 & $\mathrm{H}$ & -0.10529 & -2.54314 & 2.697579 & $\mathrm{H}$ & -1.63433 & -1.06029 & 2.697526 \\
\hline $\mathrm{H}$ & 0.751738 & -2.40648 & -3.01043 & $\mathrm{H}$ & -1.56069 & -1.58351 & 2.41799 & $\mathrm{H}$ & -0.40832 & -2.20455 & 3.248888 \\
\hline $\mathrm{H}$ & -2.15077 & 4.194319 & -0.72389 & $\mathrm{H}$ & -0.26681 & 2.944695 & -1.57168 & $\mathrm{H}$ & 1.879072 & 4.665493 & -0.40613 \\
\hline $\mathrm{H}$ & -2.12846 & 4.094675 & 1.0431 & $\mathrm{H}$ & 1.021327 & 4.151753 & -1.43433 & $\mathrm{H}$ & 0.215052 & 4.047978 & -0.43458 \\
\hline $\mathrm{H}$ & -0.60542 & 4.044214 & 0.13533 & $\mathrm{H}$ & -0.34777 & 4.162922 & -0.30889 & $\mathrm{H}$ & 0.918423 & 4.633057 & 1.076579 \\
\hline
\end{tabular}




\begin{tabular}{|c|c|c|c|c|c|c|c|c|c|c|c|}
\hline $\mathrm{H}$ & 4.702002 & -0.439 & 0.773853 & $\mathrm{H}$ & -5.34333 & -0.1105 & 0.224396 & $\mathrm{H}$ & -4.36791 & 0.478428 & -1.92118 \\
\hline $\mathrm{H}$ & 4.949662 & 1.136193 & -0.03097 & $\mathrm{H}$ & -5.06542 & 0.023229 & -1.49656 & $\mathrm{H}$ & -4.4554 & -0.96588 & -0.86391 \\
\hline $\mathrm{H}$ & 4.529452 & 1.103947 & 1.665802 & $\mathrm{H}$ & -4.76181 & -1.53936 & -0.67584 & $\mathrm{H}$ & -4.89345 & 0.65624 & -0.26251 \\
\hline $\mathrm{H}$ & -2.92256 & -2.81419 & -0.21013 & $\mathrm{H}$ & 1.99899 & -2.66966 & -1.04268 & $\mathrm{H}$ & 3.317074 & -2.41729 & 0.473568 \\
\hline $\mathrm{H}$ & -1.43895 & -3.44278 & 0.478078 & $\mathrm{H}$ & 1.538538 & -1.03977 & -1.45273 & $\mathrm{H}$ & 1.942366 & -3.40958 & 0.0596 \\
\hline $\mathrm{H}$ & -0.47432 & -2.29021 & 2.57937 & $\mathrm{H}$ & 4.395753 & -3.20477 & -0.39468 & $\mathrm{H}$ & 1.014237 & -3.22713 & -2.25113 \\
\hline $\mathrm{H}$ & -1.99739 & -3.11105 & 2.874602 & $\mathrm{H}$ & 4.296019 & -2.74489 & -2.08815 & $\mathrm{H}$ & 2.734927 & -3.56597 & -2.31697 \\
\hline $\mathrm{H}$ & -1.75798 & -1.45891 & 3.479461 & $\mathrm{H}$ & 5.556084 & -2.03531 & -1.0582 & $\mathrm{H}$ & 2.052018 & -2.29258 & -3.34764 \\
\hline
\end{tabular}

$1 b 3$

\begin{tabular}{|l|l|l|l|}
\hline $\mathrm{C}$ & -3.15541 & -1.67838 & -0.49002 \\
\hline $\mathrm{C}$ & -2.65181 & -0.27718 & -0.30757 \\
\hline $\mathrm{C}$ & -1.14797 & -0.32471 & -0.4245 \\
\hline $\mathrm{C}$ & -0.80379 & -1.75309 & 0.159469 \\
\hline
\end{tabular}

\begin{tabular}{|c|r|r|r|}
\hline $\mathrm{C}$ & -1.92133 & -2.58278 & -0.52503 \\
\hline $\mathrm{C}$ & -0.4346 & 0.904872 & 0.146992 \\
\hline
\end{tabular}

\begin{tabular}{|r|r|r|r|}
\hline $\mathrm{C}$ & -0.4346 & 0.904872 & 0.146992 \\
\hline $\mathrm{C}$ & 0.82293 & 1.28317 & -0.65108 \\
\hline $\mathrm{C}$ & 0.603653 & -2.26183 & -0.22734 \\
\hline
\end{tabular}

\begin{tabular}{|r|r|r|r|}
\hline $\mathrm{C}$ & 1.8025 & 2.22486 & 0.082716 \\
\hline $\mathrm{C}$ & 2.816333 & 1.458958 & 0.985966 \\
\hline
\end{tabular}

\begin{tabular}{|l|r|r|r|}
\hline $\mathrm{C}$ & 2.816333 & 1.458958 & 0.985966 \\
\hline $\mathrm{C}$ & 3.91934 & 0.724182 & 0.21927 \\
\hline
\end{tabular}

\begin{tabular}{|c|c|c|c|}
\hline $\mathrm{C}$ & 3.948972 & -0.72478 & -0.13392 \\
\hline $\mathrm{C}$ & -3.35323 & 0.832968 & -0.01275 \\
\hline
\end{tabular}

\begin{tabular}{|l|l|l|l|}
\hline $\mathrm{C}$ & -3.35323 & 0.832968 & -0.01275 \\
\hline $\mathrm{O}$ & -1.25381 & 2.110615 & 0.114012 \\
\hline $\mathrm{C}$ & -2.60884 & 2.123476 & 0.146892 \\
\hline
\end{tabular}

\begin{tabular}{|r|r|r|r|}
\hline $\mathrm{C}$ & -1.02628 & -1.8336 & 1.685038 \\
\hline $\mathrm{O}$ & -3.18179 & 3.184059 & 0.26891 \\
\hline
\end{tabular}

\begin{tabular}{|r|r|r|r|}
\hline $\mathrm{O}$ & -3.18179 & 3.184059 & 0.26891 \\
\hline $\mathrm{O}$ & 4.891636 & 1.3838 & -0.13884 \\
\hline $\mathrm{C}$ & 2.505126 & 3.169369 & -0.90379 \\
\hline $\mathrm{C}$ & -4.84152 & 0.922526 & 0.147597 \\
\hline $\mathrm{C}$ & 1.751451 & -1.74885 & 0.683838 \\
\hline $\mathrm{C}$ & 3.113296 & -1.77511 & 0.033412 \\
\hline $\mathrm{C}$ & 3.55546 & -3.13754 & -0.44746 \\
\hline $\mathrm{H}$ & -0.90804 & -0.37704 & -1.49968 \\
\hline $\mathrm{O}$ & -4.31009 & -2.04881 & -0.58478 \\
\hline $\mathrm{H}$ & -1.67056 & -2.77868 & -1.57777 \\
\hline $\mathrm{H}$ & -2.12309 & -3.54651 & -0.04996 \\
\hline $\mathrm{H}$ & -0.19791 & 0.746029 & 1.206102 \\
\hline $\mathrm{H}$ & 0.458671 & 1.769809 & -1.56307 \\
\hline $\mathrm{H}$ & 1.343146 & 0.37846 & -0.98084 \\
\hline $\mathrm{H}$ & 0.594641 & -3.35808 & -0.2036 \\
\hline $\mathrm{H}$ & 0.800302 & -1.98409 & -1.27051 \\
\hline $\mathrm{H}$ & 1.207472 & 2.849158 & 0.761147 \\
\hline $\mathrm{H}$ & 2.285129 & 0.803656 & 1.68142 \\
\hline
\end{tabular}




\begin{tabular}{|c|r|r|r|}
\hline $\mathrm{H}$ & 3.347236 & 2.200198 & 1.59092 \\
\hline $\mathrm{H}$ & 4.895436 & -0.93328 & -0.62961 \\
\hline $\mathrm{H}$ & -2.0475 & -1.55286 & 1.959299 \\
\hline $\mathrm{H}$ & -0.86495 & -2.86095 & 2.028692 \\
\hline $\mathrm{H}$ & -0.34967 & -1.18924 & 2.250989 \\
\hline $\mathrm{H}$ & 3.225499 & 3.81363 & -0.39243 \\
\hline $\mathrm{H}$ & 3.056436 & 2.619225 & -1.67215 \\
\hline $\mathrm{H}$ & 1.765673 & 3.804609 & -1.40199 \\
\hline $\mathrm{H}$ & -5.24498 & 1.64219 & -0.57212 \\
\hline $\mathrm{H}$ & -5.31305 & -0.04713 & 0.002354 \\
\hline $\mathrm{H}$ & -5.08905 & 1.318553 & 1.138258 \\
\hline $\mathrm{H}$ & 1.790989 & -2.38102 & 1.581467 \\
\hline $\mathrm{H}$ & 1.522708 & -0.74805 & 1.03395 \\
\hline $\mathrm{H}$ & 3.533064 & -3.86204 & 0.377114 \\
\hline $\mathrm{H}$ & 2.882285 & -3.52689 & -1.22062 \\
\hline $\mathrm{H}$ & 4.5668 & -3.11612 & -0.8582 \\
\hline
\end{tabular}

\begin{tabular}{|c|c|c|c|c|c|c|c|c|c|c|c|c|c|c|c|c|}
\hline & & ional & & & Bas & & $\frac{G}{\text { Type }}$ & $c$ of $\mathrm{Da}$ & 35 & & & & & & & \\
\hline & B31 & Lin & & & 6-311. & (d,p) & Unsea & led Shifts & 36 & $\mathrm{H}$ & & 1.57 & 1.47 & 2.61 & 1.68 & 1.21 \\
\hline 3 & & & & & & & & & 37 & $\mathrm{H}$ & & 1.79 & 1.91 & 1.12 & 1.68 & 1.80 \\
\hline 12 & Nuclei & sp2? & $\frac{\text { DPpit }}{\text { xpenta }}$ & $\begin{array}{l}0.00 \mathrm{~W} \\
\text { Isomer 1 }\end{array}$ & $\begin{array}{l}0.00 \% \\
\text { Isomer } 2 \\
\end{array}$ & $\begin{array}{l}100.00 \% \\
\text { Isomer } 3 \\
\end{array}$ & $\begin{array}{l}0.00 \% \\
\text { Isomer }\end{array}$ & 4 Isomer 5 & 38 & $\mathrm{H}$ & & 2.04 & 1.85 & 2.99 & 1.92 & 1.84 \\
\hline 15 & c & & & 59.3 & & 583 & 58.8 & & 39 & $\mathrm{H}$ & & 2.35 & 2.89 & 2.24 & 2.95 & 3.31 \\
\hline 16 & c & & 27.7 & 37.1 & 37.4 & 32.8 & 46.0 & & 40 & $\mathrm{H}$ & & 5.99 & 6.30 & 6.56 & 6.51 & 6.46 \\
\hline $\begin{array}{l}17 \\
18\end{array}$ & c & & $\begin{array}{l}28.4 \\
1474\end{array}$ & $\begin{array}{l}31.9 \\
1666\end{array}$ & $\begin{array}{l}35.5 \\
171.6\end{array}$ & $\begin{array}{l}30.8 \\
1064\end{array}$ & $\begin{array}{l}322 \\
1256\end{array}$ & & 41 & $\mathrm{H}$ & & 2.37 & 1.92 & 2.72 & 2.13 & 2.47 \\
\hline $\begin{array}{l}10 \\
19\end{array}$ & c & $x_{x}$ & 131.2 & 135.1 & 134.9 & $\begin{array}{l}10.4 \\
138.8\end{array}$ & $\begin{array}{l}132.0 \\
137.1\end{array}$ & & 42 & $\mathrm{H}$ & & 2.50 & 2.76 & 2.51 & 2.54 & 2.55 \\
\hline 20 & c & $\mathrm{x}$ & 208.0 & 221.0 & 218.6 & 220.3 & 221.9 & & 43 & $\mathrm{H}$ & & 2.17 & 2.84 & 2.59 & 2.27 & 2.77 \\
\hline 21 & c & & 49.6 & 56.6 & 52.6 & 53.0 & 55.1 & & 44 & $\mathrm{H}$ & & 1.94 & 1.96 & 1.47 & 1.48 & 1.17 \\
\hline $\begin{array}{l}22 \\
23\end{array}$ & $\begin{array}{l}\mathrm{c} \\
\mathrm{c}\end{array}$ & & $\begin{array}{l}34.1 \\
35.4\end{array}$ & $\begin{array}{l}34.3 \\
40.4\end{array}$ & $\begin{array}{l}379 \\
401\end{array}$ & 44.7 & $\begin{array}{l}34.0 \\
453\end{array}$ & & 45 & $\mathrm{H}$ & & 1.86 & 1.66 & 1.95 & 1.48 & 1.56 \\
\hline 24 & c & & $\begin{array}{l}32.4 \\
82.1\end{array}$ & 86.4 & $\begin{array}{l}+0.1 \\
832\end{array}$ & $\begin{array}{l}39.4 \\
89.4\end{array}$ & $\begin{array}{l}73.3 \\
83.9\end{array}$ & & 46 & $\mathrm{H}$ & $\mathrm{x}$ & 4.53 & 4.49 & 5.42 & 4.43 & 5.50 \\
\hline 25 & c & & 86.7 & 97.7 & 98.5 & 95.5 & 98.1 & & 47 & $\mathrm{H}$ & & 2.50 & 2.73 & 2.54 & 2.83 & 2.69 \\
\hline${ }^{26}$ & c & $\mathrm{x}$ & 153.7 & $\begin{array}{l}168.4 \\
307\end{array}$ & $\begin{array}{l}166.8 \\
3.6\end{array}$ & $\begin{array}{l}167.5 \\
308\end{array}$ & $\begin{array}{l}165.0 \\
311\end{array}$ & & 48 & $\mathrm{H}$ & & 2.50 & 2.65 & 2.70 & 2.62 & 2.45 \\
\hline $\begin{array}{l}27 \\
28 \\
28\end{array}$ & $\begin{array}{c}c \\
c\end{array}$ & & $\begin{array}{l}25.5 \\
385\end{array}$ & $\begin{array}{l}30.7 \\
443\end{array}$ & $\begin{array}{l}31.6 \\
473\end{array}$ & $\begin{array}{l}30.8 \\
444\end{array}$ & $\begin{array}{l}31.1 \\
462\end{array}$ & & 49 & $\mathrm{H}$ & & 1.46 & 1.76 & 1.55 & 1.59 & 1.38 \\
\hline 29 & c & & 21.5 & 22.5 & 24,3 & 22.6 & 23.9 & & 50 & $\mathrm{H}$ & & 2.06 & 1.92 & 1.61 & 2.18 & 1.78 \\
\hline 30 & c & & 22.1 & 25.4 & 31.5 & 25.2 & 27.6 & & 51 & $\mathrm{H}$ & & 0.89 & 0.87 & 1.24 & 0.84 & 1.20 \\
\hline $\begin{array}{l}31 \\
32\end{array}$ & c & & $\begin{array}{l}232 . \\
1223\end{array}$ & $\begin{array}{l}26.1 \\
1706\end{array}$ & 24.7 & 24.5 & $\begin{array}{l}25.5 \\
1353\end{array}$ & & 52 & $\mathrm{H}$ & & 1.77 & 1.98 & 2.08 & 1.93 & 1.98 \\
\hline $\begin{array}{l}32 \\
33 \\
33\end{array}$ & $\begin{array}{l}c \\
c \\
c\end{array}$ & $\mathrm{x}$ & $\begin{array}{l}126.3 \\
12.8\end{array}$ & 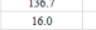 & $\begin{array}{l}134.6 \\
10.5\end{array}$ & $\begin{array}{l}136.7 \\
15.7\end{array}$ & $\begin{array}{l}133.3 \\
16.5\end{array}$ & & 53 & $\mathrm{H}$ & & 1.20 & 1.31 & 1.16 & 1.31 & 0.96 \\
\hline 34 & c & $x$ & 165.2 & 174,2 & 172.1 & 1728 & 171.8 & & 54 & H & & 1.81 & 1.88 & 1.82 & 1.91 & 1.84 \\
\hline . & & A & & B & & $\mathrm{C}$ & & D & & & E & $\mathrm{F}$ & & G & & $\mathrm{H}$ \\
\hline 1 & & & nctio & nal & & & Solv & ent? & & & Basis & s Set & & Type o & f $D$ & ta \\
\hline 2 & & & B3LYF & & & & $\mathrm{PC}$ & $\mathrm{CM}$ & & & $6-311+$ & $-G(d, p)$ & & Unscaled & $\mathrm{d} \mathrm{Sh}$ & ifts \\
\hline 3 & & & & & & & & & & & & & & & & \\
\hline 4 & & & & & & Isome & r 1 & Isome & $\mathrm{r} 2$ & & mer 3 & Isom & 4 & Isomer 5 & Is & mer 6 \\
\hline 5 & & $\mathrm{sDP}$ & $+(\mathrm{H}$ & data) & & 20.5 & $5 \%$ & ती 0.00 & & -17 & $.21 \%$ & 네 0.2 & & - & & - \\
\hline 6 & & $\mathrm{sDP}$ & $+\quad(C$ & data) & & 0.46 & & 0.00 & & - & $.54 \%$ & ती 0.0 & & - & & - \\
\hline 7 & & SDP4- & (a11 & data) & & 0.12 & & 페 0.00 & & & 7. $88 \%$ & 政 0.0 & & - & & - \\
\hline 8 & & $\mathrm{uDP}$ & $+\quad(\mathrm{H}$ & data) & & $\rrbracket 12.6$ & $0 \%$ & 0.00 & & -1 & $7.40 \%$ & 페 0.0 & & - & & - \\
\hline 9 & & $\mathrm{uDP}<$ & $+\quad(C$ & data) & & 1.39 & & - 0.00 & & -llg & $60 \%$ & 제 0.0 & & - & & - \\
\hline 10 & & 1DP4- & (a11 & data) & - & 0.20 & & - 0.00 & & - 9 & 9. $80 \%$ & 政 0.0 & & - & & - \\
\hline 11 & & DP4 & $+\quad\left(\mathrm{H} \mathrm{c}^{\circ}\right.$ & lata) & & 3.60 & & 페 0.00 & & -19 & $40 \%$ & 제 0.0 & & - & & - \\
\hline 12 & & DP4 & $+\quad(C$ d & lata) & & 0.01 & & 페 0.00 & & & $9.99 \%$ & 메 0.0 & & - & & - \\
\hline 13 & & DP4+ & (a11 & data) & ш & 0.00 & & - 0.00 & & -1 & $0.00 \%$ & 政 0.0 & & - & & - \\
\hline
\end{tabular}

Figure S2. Detailed DP4+ probability (calculated at PCM/b3lyp/6-311+G(d,p) level) for compound 2. Isomer 1 is $(1 R, 8 R, 10 R, 11 R)-\mathbf{2 a}$, isomer 2 is $(1 R, 8 R, 10 R, 11 S)-\mathbf{2 b}$, isomer 3 is $(1 R, 8 S, 10 R$, $11 R)-\mathbf{2 c}$, and isomer 4 is $(1 R, 8 S, 10 R, 11 S)-\mathbf{2 d}$. 


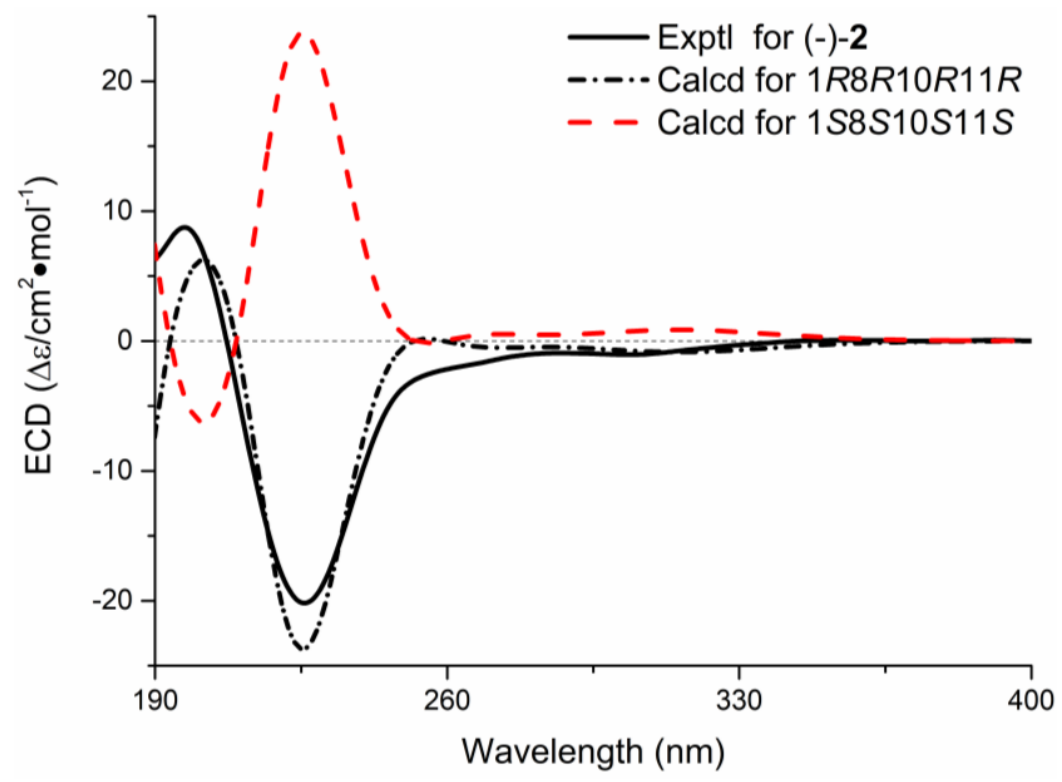

Figure S3. Experimental and calculated ECD spectra of 2

Table S4. Stable conformers of compound $\mathbf{2}$ with $(1 R, 8 R, 10 R, 11 R)-\mathbf{2 a},(1 R, 8 R, 10 R, 11 S)-\mathbf{2 b}$, $(1 R, 8 S, 10 R, 11 R)-\mathbf{2} \mathbf{c}$ and $(1 R, 8 S, 10 R, 11 S)-\mathbf{2 d}$ configurations, respectively.

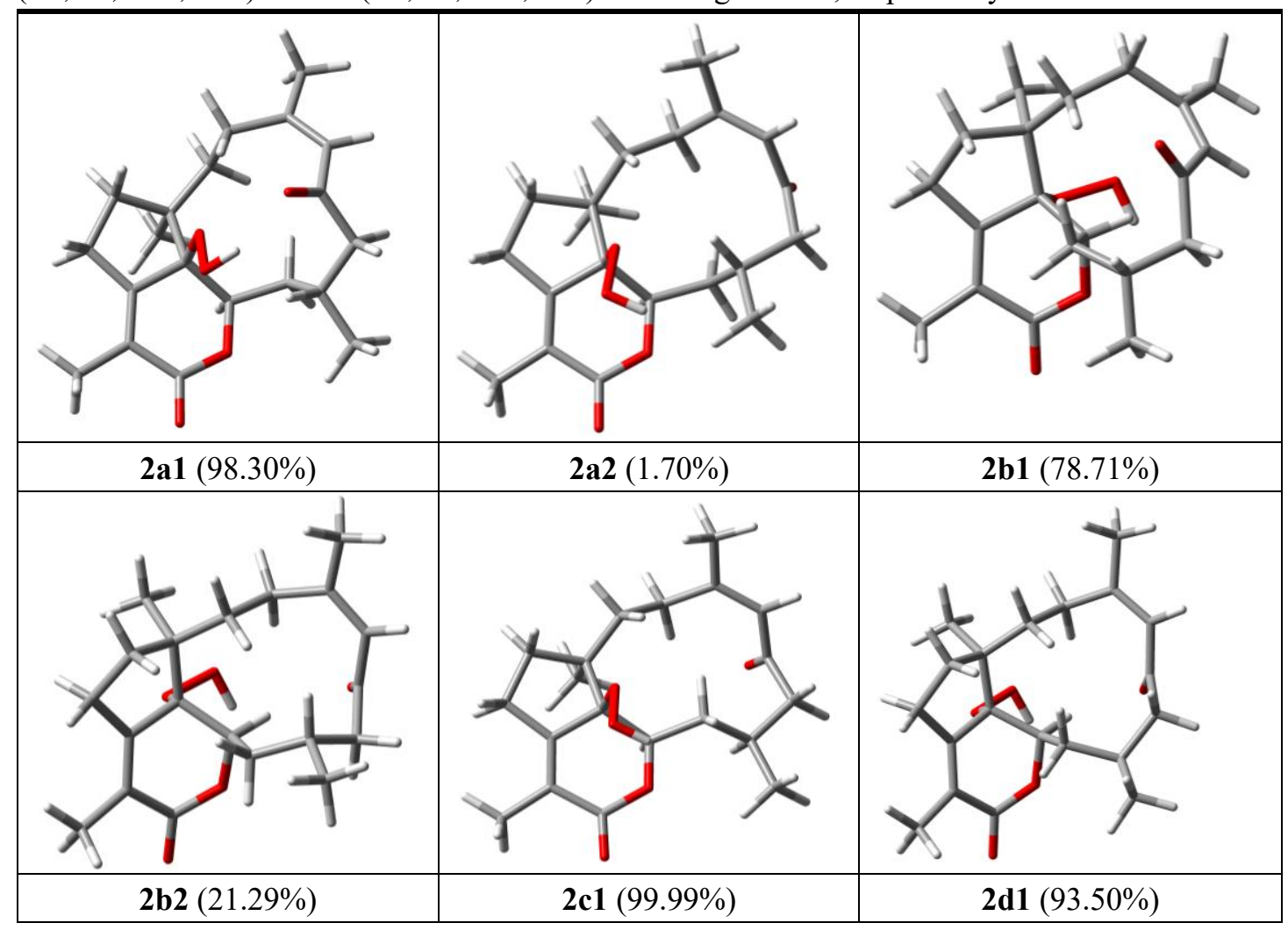




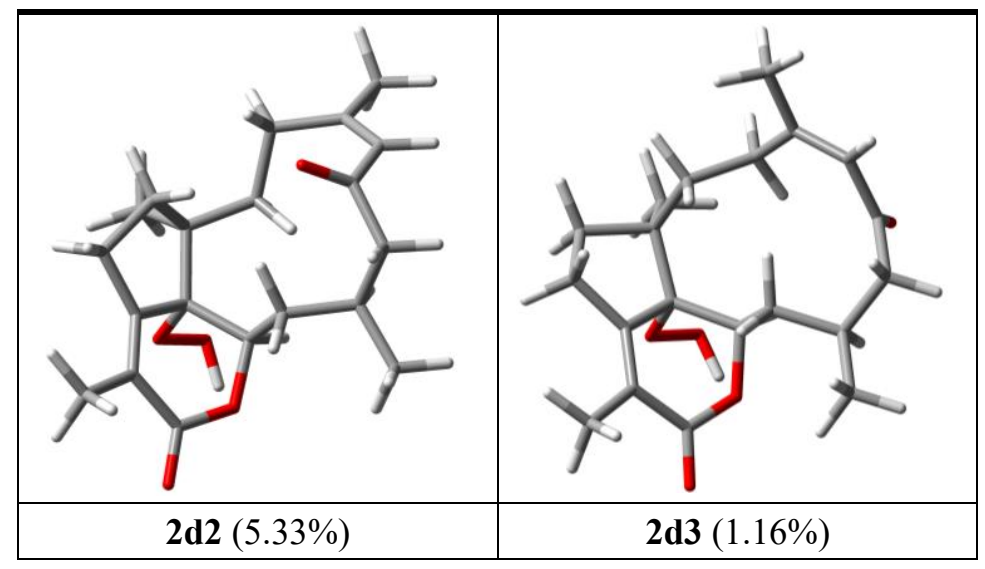

Table S5. Important thermodynamic parameters (a.u.) of the optimized compound $\mathbf{2}$ with simplified structures at B3LYP/6-31+G(d,p) level in the gas phase.

\begin{tabular}{|c|c|c|}
\hline NO. & E + ZPE & G \\
\hline $\mathbf{2 a 1}$ & -1154.576945 & -1154.627777 \\
\hline $\mathbf{2 a 2}$ & -1154.571802 & -1154.623950 \\
\hline $\mathbf{2 b 1}$ & -1154.568116 & -1154.618831 \\
\hline $\mathbf{2 b 2}$ & -1154.566128 & -1154.617597 \\
\hline $\mathbf{2 c 1}$ & -1154.575948 & -1154.628798 \\
\hline $\mathbf{2 d} \mathbf{2 d 2}$ & -1154.573106 & -1154.624731 \\
\hline $\mathbf{2 d 3}$ & -1154.570710 & -1154.622029 \\
\hline & -1154.569014 & -1154.620590 \\
\hline
\end{tabular}

Table S6. Optimized Z-Matrixes of compound 2 with simplified structures in the Gas Phase $(\AA)$ at B3LYP/6-31+G(d,p) level.

\begin{tabular}{|l|c|c|c|c|c|c|c|c|c|c|c|}
\hline \multicolumn{9}{|c|}{$\mathbf{2 a 1}$} & \multicolumn{7}{|c|}{$\mathbf{2 a 2}$} & \multicolumn{4}{|c|}{$\mathbf{2 b 1}$} \\
\hline C & -2.07772 & -2.54047 & -0.72657 & C & -2.52097 & -2.46998 & -0.13694 & C & 2.136987 & -2.09436 & 1.493834 \\
\hline C & -2.15565 & -1.0975 & -0.2713 & C & -2.42111 & -0.97132 & 0.018542 & C & 2.090258 & -1.04623 & 0.418355 \\
\hline C & -0.76558 & -0.61618 & 0.093428 & C & -0.9647 & -0.52042 & -0.05724 & C & 0.671958 & -0.9091 & -0.13041 \\
\hline C & -0.07516 & -1.95948 & 0.600302 & C & -0.16639 & -1.84282 & 0.343335 & C & -0.22924 & -1.87236 & 0.769089 \\
\hline C & -0.61533 & -2.96824 & -0.45136 & C & -1.06708 & -2.92337 & -0.32823 & C & 0.688931 & -2.09365 & 2.013964 \\
\hline C & -0.83299 & 0.567419 & 1.078277 & C & -0.83241 & 0.757851 & 0.801348 & C & 0.374238 & 0.602402 & -0.24623 \\
\hline C & 0.454176 & 1.385904 & 1.305452 & C & 0.490132 & 1.524694 & 0.92833 & C & 0.334981 & 1.420684 & 1.053175 \\
\hline C & 1.474339 & -1.98153 & 0.739119 & C & 1.264196 & -2.00324 & -0.26241 & C & -1.61736 & -1.40289 & 1.302557 \\
\hline C & 0.735243 & 2.543454 & 0.304165 & C & 1.224996 & 2.049814 & -0.32841 & C & -0.31104 & 2.831668 & 0.966422 \\
\hline C & 2.227153 & 2.59418 & -0.11912 & C & 2.689687 & 2.448974 & 0.032096 & C & -1.65374 & 2.867398 & 0.186777 \\
\hline C & 2.638828 & 1.343939 & -0.89372 & C & 3.454353 & 1.274898 & 0.618295 & C & -2.56984 & 1.688252 & 0.519102 \\
\hline C & 3.655709 & 0.464696 & -0.25974 & C & 3.971634 & 0.249949 & -0.33741 & C & -3.02014 & 0.87366 & -0.63404 \\
\hline C & -3.23243 & -0.30133 & -0.18619 & C & -3.42746 & -0.10273 & 0.21046 & C & 3.045188 & -0.21456 & -0.03042 \\
\hline O & -1.86786 & 1.516168 & 0.731453 & O & -1.8263 & 1.729998 & 0.372932 & O & 1.358857 & 1.171779 & -1.15719 \\
\hline C & -3.0703 & 1.116159 & 0.237663 & C & -3.13159 & 1.351845 & 0.239106 & C & 2.675967 & 0.826412 & -1.03855 \\
\hline C & -0.63756 & -2.34512 & 1.990118 & C & -0.17673 & -2.05198 & 1.86941 & C & -0.39239 & -3.22876 & 0.048061 \\
\hline O & -3.96068 & 1.934169 & 0.141385 & O & -3.97853 & 2.208806 & 0.11101 & O & 3.491473 & 1.393251 & -1.73212 \\
\hline
\end{tabular}




\begin{tabular}{|c|c|c|c|c|c|c|c|c|c|c|c|}
\hline $\mathrm{O}$ & 2.119058 & 1.09168 & -1.97797 & $\mathrm{O}$ & 3.561257 & 1.139158 & 1.829918 & $\mathrm{O}$ & -2.85256 & 1.428142 & 1.683592 \\
\hline $\mathrm{C}$ & 0.294968 & 3.894926 & 0.882855 & $\mathrm{C}$ & 0.533331 & 3.268621 & -0.96287 & $\mathrm{C}$ & 0.644107 & 3.89636 & 0.40726 \\
\hline $\mathrm{C}$ & -4.62953 & -0.69698 & -0.57742 & $\mathrm{C}$ & -4.88379 & -0.46917 & 0.279318 & $\mathrm{C}$ & 4.493751 & -0.23655 & 0.368713 \\
\hline $\mathrm{C}$ & 2.392547 & -1.71496 & -0.5014 & $\mathrm{C}$ & 2.509664 & -1.57745 & 0.567067 & $\mathrm{C}$ & -2.89964 & -1.45501 & 0.435263 \\
\hline $\mathrm{C}$ & 3.590322 & -0.87507 & -0.10375 & $\mathrm{C}$ & 3.603684 & -1.04351 & -0.33649 & $\mathrm{C}$ & -3.14718 & -0.4695 & -0.69669 \\
\hline $\mathrm{C}$ & 4.694058 & -1.607 & 0.616727 & $\mathrm{C}$ & 4.201582 & -2.02057 & -1.31703 & $\mathrm{C}$ & -3.61009 & -1.10644 & -1.98215 \\
\hline $\mathrm{O}$ & -0.04187 & -0.36113 & -1.11267 & $\mathrm{O}$ & -0.56762 & -0.31255 & -1.44216 & $\mathrm{O}$ & 0.833151 & -1.42394 & -1.47884 \\
\hline $\mathrm{O}$ & -0.68496 & 0.666351 & -1.92684 & $\mathrm{O}$ & -1.53939 & 0.468727 & -2.17941 & $\mathrm{O}$ & -0.37087 & -1.17455 & -2.26089 \\
\hline $\mathrm{H}$ & -2.34097 & -2.64112 & -1.78495 & $\mathrm{H}$ & -2.97292 & -2.91653 & 0.757647 & $\mathrm{H}$ & 2.873947 & -1.87601 & 2.271709 \\
\hline $\mathrm{H}$ & -2.7925 & -3.15677 & -0.16907 & $\mathrm{H}$ & -3.1607 & -2.75184 & -0.98012 & $\mathrm{H}$ & 2.398019 & -3.07326 & 1.070198 \\
\hline $\mathrm{H}$ & -0.02653 & -2.90064 & -1.36823 & $\mathrm{H}$ & -0.86688 & -3.91304 & 0.093861 & $\mathrm{H}$ & 0.424906 & -3.01588 & 2.541211 \\
\hline $\mathrm{H}$ & -0.54316 & -3.99677 & -0.08366 & $\mathrm{H}$ & -0.82977 & -2.9637 & -1.39505 & $\mathrm{H}$ & 0.559045 & -1.26786 & 2.724126 \\
\hline $\mathrm{H}$ & -1.12849 & 0.134377 & 2.040561 & $\mathrm{H}$ & -1.10609 & 0.457717 & 1.820752 & $\mathrm{H}$ & -0.56259 & 0.708996 & -0.78822 \\
\hline $\mathrm{H}$ & 1.290873 & 0.684735 & 1.323353 & $\mathrm{H}$ & 1.16313 & 0.889244 & 1.506909 & $\mathrm{H}$ & 1.355404 & 1.523619 & 1.436691 \\
\hline $\mathrm{H}$ & 0.397879 & 1.808818 & 2.315257 & $\mathrm{H}$ & 0.272334 & 2.377307 & 1.584852 & $\mathrm{H}$ & -0.21185 & 0.853932 & 1.807085 \\
\hline $\mathrm{H}$ & 1.744193 & -1.27986 & 1.535959 & $\mathrm{H}$ & 1.373606 & -3.06777 & -0.49908 & $\mathrm{H}$ & -1.54773 & -0.42576 & 1.772903 \\
\hline $\mathrm{H}$ & 1.719573 & -2.97032 & 1.146674 & $\mathrm{H}$ & 1.273858 & -1.49071 & -1.22656 & $\mathrm{H}$ & -1.8293 & -2.08983 & 2.132954 \\
\hline $\mathrm{H}$ & 0.146979 & 2.367448 & -0.59665 & $\mathrm{H}$ & 1.272812 & 1.24553 & -1.06975 & $\mathrm{H}$ & -0.54684 & 3.099167 & 2.003254 \\
\hline $\mathrm{H}$ & 2.372001 & 3.458444 & -0.77911 & $\mathrm{H}$ & 2.6802 & 3.254545 & 0.77418 & $\mathrm{H}$ & -1.46061 & 2.904324 & -0.89109 \\
\hline $\mathrm{H}$ & 2.866514 & 2.736223 & 0.760179 & $\mathrm{H}$ & 3.181974 & 2.814859 & -0.87705 & $\mathrm{H}$ & -2.17545 & 3.797065 & 0.447418 \\
\hline $\mathrm{H}$ & 4.486806 & 0.995626 & 0.20453 & $\mathrm{H}$ & 4.659159 & 0.611291 & -1.10209 & $\mathrm{H}$ & -3.18174 & 1.437022 & -1.55266 \\
\hline $\mathrm{H}$ & -0.34495 & -3.37288 & 2.227092 & $\mathrm{H}$ & -1.19235 & -2.03176 & 2.276658 & $\mathrm{H}$ & -0.93515 & -3.91837 & 0.704114 \\
\hline $\mathrm{H}$ & -0.24439 & -1.70478 & 2.785901 & $\mathrm{H}$ & 0.253816 & -3.03029 & 2.107505 & $\mathrm{H}$ & -0.94515 & -3.13297 & -0.88687 \\
\hline $\mathrm{H}$ & -1.72928 & -2.29382 & 2.028959 & $\mathrm{H}$ & 0.408219 & -1.30034 & 2.405136 & $\mathrm{H}$ & 0.570191 & -3.68227 & -0.198 \\
\hline $\mathrm{H}$ & -0.76507 & 3.86296 & 1.146159 & $\mathrm{H}$ & -0.52023 & 3.082402 & -1.1856 & $\mathrm{H}$ & 0.173724 & 4.88582 & 0.416314 \\
\hline $\mathrm{H}$ & 0.441591 & 4.702855 & 0.15788 & $\mathrm{H}$ & 0.551836 & 4.122542 & -0.27599 & $\mathrm{H}$ & 1.555925 & 3.957332 & 1.011167 \\
\hline $\mathrm{H}$ & 0.866593 & 4.14698 & 1.785135 & $\mathrm{H}$ & 1.040519 & 3.569831 & -1.88548 & $\mathrm{H}$ & 0.941938 & 3.667155 & -0.6197 \\
\hline $\mathrm{H}$ & -5.33704 & -0.46801 & 0.225462 & $\mathrm{H}$ & -5.02751 & -1.55042 & 0.320297 & $\mathrm{H}$ & 5.119986 & -0.37416 & -0.51803 \\
\hline $\mathrm{H}$ & -4.95648 & -0.1207 & -1.44903 & $\mathrm{H}$ & -5.4153 & -0.07721 & -0.59408 & $\mathrm{H}$ & 4.79514 & 0.7185 & 0.812909 \\
\hline $\mathrm{H}$ & -4.6958 & -1.76014 & -0.81721 & $\mathrm{H}$ & -5.35644 & -0.01604 & 1.156391 & $\mathrm{H}$ & 4.706677 & -1.0376 & 1.07876 \\
\hline $\mathrm{H}$ & 1.845006 & -1.21703 & -1.29341 & $\mathrm{H}$ & 2.265424 & -0.83015 & 1.318103 & $\mathrm{H}$ & -3.73172 & -1.32675 & 1.139904 \\
\hline $\mathrm{H}$ & 2.746955 & -2.67355 & -0.89847 & $\mathrm{H}$ & 2.88806 & -2.44446 & 1.12356 & $\mathrm{H}$ & -3.01016 & -2.46501 & 0.028262 \\
\hline $\mathrm{H}$ & 5.498336 & -0.93974 & 0.936289 & $\mathrm{H}$ & 3.43904 & -2.45067 & -1.97693 & $\mathrm{H}$ & -2.82726 & -1.75882 & -2.38455 \\
\hline $\mathrm{H}$ & 4.310229 & -2.13226 & 1.500446 & $\mathrm{H}$ & 4.660606 & -2.8612 & -0.78083 & $\mathrm{H}$ & -3.8736 & -0.36636 & -2.74216 \\
\hline $\mathrm{H}$ & 5.123312 & -2.37667 & -0.03732 & $\mathrm{H}$ & 4.968258 & -1.55409 & -1.94053 & $\mathrm{H}$ & -4.48961 & -1.73644 & -1.79676 \\
\hline $\mathrm{H}$ & 0.147752 & 1.087604 & -2.23079 & $\mathrm{H}$ & -1.20619 & 1.371325 & -2.03681 & $\mathrm{H}$ & -0.06083 & -0.42769 & -2.80187 \\
\hline \multicolumn{4}{|c|}{$2 \mathrm{~b} 2$} & \multicolumn{4}{|c|}{$2 \mathrm{c1}$} & \multicolumn{4}{|c|}{$2 d 1$} \\
\hline $\mathrm{C}$ & 2.404723 & -1.88207 & 1.488562 & $\mathrm{C}$ & 2.668693 & -2.38911 & -0.24569 & $\mathrm{C}$ & 2.184139 & -2.03902 & 1.570743 \\
\hline $\mathrm{C}$ & 2.340803 & -0.75214 & 0.498714 & $\mathrm{C}$ & 2.459145 & -0.89472 & -0.14298 & $\mathrm{C}$ & 2.227701 & -0.94895 & 0.536241 \\
\hline $\mathrm{C}$ & 1.009933 & -0.77681 & -0.24765 & $\mathrm{C}$ & 1.017634 & -0.61325 & 0.226162 & $\mathrm{C}$ & 0.91727 & -0.90425 & -0.24173 \\
\hline $\mathrm{C}$ & 0.154514 & -1.92101 & 0.455672 & $\mathrm{C}$ & 0.256055 & -1.84567 & -0.43022 & $\mathrm{C}$ & -0.03795 & -1.95739 & 0.480279 \\
\hline $\mathrm{C}$ & 0.918225 & -2.13759 & 1.798158 & $\mathrm{C}$ & 1.256215 & -2.98708 & -0.07467 & $\mathrm{C}$ & 0.675925 & -2.16638 & 1.851543 \\
\hline $\mathrm{C}$ & 0.496254 & 0.68225 & -0.32386 & $\mathrm{C}$ & 0.610669 & 0.811762 & -0.17879 & $\mathrm{C}$ & 0.513661 & 0.578397 & -0.39801 \\
\hline
\end{tabular}




\begin{tabular}{|c|c|c|c|c|c|c|c|c|c|c|c|}
\hline $\mathrm{C}$ & 0.176397 & 1.444878 & 0.990669 & $\mathrm{C}$ & -0.67957 & 1.30772 & 0.475148 & $\mathrm{C}$ & 0.192302 & 1.423413 & 0.850265 \\
\hline $\mathrm{C}$ & -1.32649 & -1.59986 & 0.760895 & $\mathrm{C}$ & -1.14948 & -2.18188 & 0.171518 & $\mathrm{C}$ & -1.50719 & -1.54936 & 0.743005 \\
\hline $\mathrm{C}$ & -1.2547 & 2.013373 & 1.217957 & $\mathrm{C}$ & -1.36955 & 2.507416 & -0.22193 & $\mathrm{C}$ & -0.53594 & 2.755255 & 0.509038 \\
\hline $\mathrm{C}$ & -1.96608 & 2.550658 & -0.05493 & $\mathrm{C}$ & -2.90923 & 2.430995 & -0.03076 & $\mathrm{C}$ & -2.07968 & 2.629104 & 0.549728 \\
\hline $\mathrm{C}$ & -2.61531 & 1.463754 & -0.91218 & $\mathrm{C}$ & -3.41727 & 1.134036 & -0.65939 & $\mathrm{C}$ & -2.65805 & 1.633529 & -0.45826 \\
\hline $\mathrm{C}$ & -3.65173 & 0.614075 & -0.24488 & $\mathrm{C}$ & -3.9405 & 0.083045 & 0.257741 & $\mathrm{C}$ & -3.7485 & 0.734137 & 0.015625 \\
\hline $\mathrm{C}$ & 3.205566 & 0.246721 & 0.259206 & $\mathrm{C}$ & 3.350129 & 0.08772 & -0.35458 & $\mathrm{C}$ & 3.169848 & -0.02559 & 0.282631 \\
\hline $\mathrm{O}$ & 1.50913 & 1.440259 & -1.05451 & $\mathrm{O}$ & 1.647909 & 1.766166 & 0.181035 & $\mathrm{O}$ & 1.597142 & 1.248818 & -1.10877 \\
\hline $\mathrm{C}$ & 2.829119 & 1.291503 & -0.74272 & $\mathrm{C}$ & 2.962109 & 1.497127 & -0.08773 & $\mathrm{C}$ & 2.893896 & 1.015736 & -0.75519 \\
\hline $\mathrm{C}$ & 0.239169 & -3.22406 & -0.36924 & $\mathrm{C}$ & 0.216405 & -1.70301 & -1.96648 & $\mathrm{C}$ & -0.01684 & -3.29434 & -0.29415 \\
\hline $\mathrm{O}$ & 3.641248 & 2.019759 & -1.26986 & $\mathrm{O}$ & 3.76132 & 2.406317 & -0.03651 & $\mathrm{O}$ & 3.77033 & 1.671081 & -1.27563 \\
\hline $\mathrm{O}$ & -2.28007 & 1.282982 & -2.07412 & $\mathrm{O}$ & -3.29105 & 0.958262 & -1.86485 & $\mathrm{O}$ & -2.21603 & 1.576209 & -1.59827 \\
\hline $\mathrm{C}$ & -1.1736 & 3.112304 & 2.290775 & $\mathrm{C}$ & -0.81476 & 3.859422 & 0.241758 & $\mathrm{C}$ & -0.08853 & 3.874513 & 1.460092 \\
\hline $\mathrm{C}$ & 4.565986 & 0.406055 & 0.876409 & $\mathrm{C}$ & 4.789725 & -0.1204 & -0.73572 & $\mathrm{C}$ & 4.525708 & 0.052187 & 0.925879 \\
\hline $\mathrm{C}$ & -2.29885 & -1.49032 & -0.44664 & $\mathrm{C}$ & -2.41857 & -1.71927 & -0.61472 & $\mathrm{C}$ & -2.45719 & -1.3644 & -0.47151 \\
\hline $\mathrm{C}$ & -3.54653 & -0.71521 & -0.0655 & $\mathrm{C}$ & -3.52201 & -1.19946 & 0.284066 & $\mathrm{C}$ & -3.70029 & -0.61089 & -0.03028 \\
\hline $\mathrm{C}$ & -4.62952 & -1.4912 & 0.639454 & $\mathrm{C}$ & -4.09555 & -2.17473 & 1.280108 & $\mathrm{C}$ & -4.8361 & -1.44605 & 0.500766 \\
\hline $\mathrm{O}$ & 1.418219 & -1.15519 & -1.5874 & $\mathrm{O}$ & 0.817584 & -0.81546 & 1.653192 & $\mathrm{O}$ & 1.329561 & -1.36802 & -1.55551 \\
\hline $\mathrm{O}$ & 0.292789 & -1.04182 & -2.50486 & $\mathrm{O}$ & 1.809698 & -0.09486 & 2.425925 & $\mathrm{O}$ & 0.236601 & -1.21425 & -2.50738 \\
\hline $\mathrm{H}$ & 2.861095 & -2.76889 & 1.029203 & $\mathrm{H}$ & 3.113442 & -2.65258 & -1.21216 & $\mathrm{H}$ & 2.581367 & -2.97629 & 1.159271 \\
\hline $\mathrm{H}$ & 2.99547 & -1.64219 & 2.376983 & $\mathrm{H}$ & 3.363028 & -2.74612 & 0.522772 & $\mathrm{H}$ & 2.770622 & -1.80712 & 2.463958 \\
\hline $\mathrm{H}$ & 0.573939 & -1.41484 & 2.548616 & $\mathrm{H}$ & 1.100546 & -3.29115 & 0.963371 & $\mathrm{H}$ & 0.372913 & -1.38098 & 2.555543 \\
\hline $\mathrm{H}$ & 0.733283 & -3.13639 & 2.20587 & $\mathrm{H}$ & 1.086716 & -3.86299 & -0.70846 & $\mathrm{H}$ & 0.404269 & -3.12653 & 2.30129 \\
\hline $\mathrm{H}$ & -0.34596 & 0.71564 & -1.00542 & $\mathrm{H}$ & 0.511801 & 0.845506 & -1.26985 & $\mathrm{H}$ & -0.31111 & 0.641955 & -1.1009 \\
\hline $\mathrm{H}$ & 0.444167 & 0.847155 & 1.868322 & $\mathrm{H}$ & -1.36118 & 0.459089 & 0.511746 & $\mathrm{H}$ & -0.3996 & 0.857697 & 1.57537 \\
\hline $\mathrm{H}$ & 0.861884 & 2.298596 & 0.998958 & $\mathrm{H}$ & -0.47732 & 1.556385 & 1.524241 & $\mathrm{H}$ & 1.139567 & 1.644435 & 1.35273 \\
\hline $\mathrm{H}$ & -1.68909 & -2.38325 & 1.439437 & $\mathrm{H}$ & -1.19333 & -3.27173 & 0.274 & $\mathrm{H}$ & -1.52789 & -0.63218 & 1.335477 \\
\hline $\mathrm{H}$ & -1.37304 & -0.67593 & 1.341227 & $\mathrm{H}$ & -1.17639 & -1.79729 & 1.194339 & $\mathrm{H}$ & -1.92982 & -2.31937 & 1.402488 \\
\hline $\mathrm{H}$ & -1.89347 & 1.220114 & 1.62232 & $\mathrm{H}$ & -1.20413 & 2.413167 & -1.30269 & $\mathrm{H}$ & -0.25457 & 3.039048 & -0.51065 \\
\hline $\mathrm{H}$ & -1.28252 & 3.123619 & -0.68958 & $\mathrm{H}$ & -3.37427 & 3.27985 & -0.54486 & $\mathrm{H}$ & -2.41232 & 2.370607 & 1.562763 \\
\hline $\mathrm{H}$ & -2.76655 & 3.22937 & 0.271388 & $\mathrm{H}$ & -3.16907 & 2.491238 & 1.032767 & $\mathrm{H}$ & -2.51809 & 3.611024 & 0.319777 \\
\hline $\mathrm{H}$ & -4.50502 & 1.14613 & 0.176174 & $\mathrm{H}$ & -4.64501 & 0.423501 & 1.016294 & $\mathrm{H}$ & -4.59147 & 1.22361 & 0.503864 \\
\hline $\mathrm{H}$ & -0.20417 & -3.11204 & -1.35891 & $\mathrm{H}$ & -0.21422 & -2.6092 & -2.40472 & $\mathrm{H}$ & -0.43428 & -3.19093 & -1.29617 \\
\hline $\mathrm{H}$ & 1.270856 & -3.55168 & -0.516 & $\mathrm{H}$ & -0.38561 & -0.85886 & -2.30925 & $\mathrm{H}$ & 0.995045 & -3.68928 & -0.40831 \\
\hline $\mathrm{H}$ & -0.29711 & -4.0159 & 0.165939 & $\mathrm{H}$ & 1.218793 & -1.57764 & -2.38535 & $\mathrm{H}$ & -0.61103 & -4.03184 & 0.257166 \\
\hline $\mathrm{H}$ & -0.65686 & 2.752565 & 3.187221 & $\mathrm{H}$ & -1.27933 & 4.684328 & -0.30872 & $\mathrm{H}$ & 0.990879 & 4.039471 & 1.384862 \\
\hline $\mathrm{H}$ & -0.62468 & 3.984939 & 1.918622 & $\mathrm{H}$ & 0.266544 & 3.908665 & 0.092654 & $\mathrm{H}$ & -0.58891 & 4.820163 & 1.226247 \\
\hline $\mathrm{H}$ & -2.17184 & 3.44591 & 2.592193 & $\mathrm{H}$ & -1.01265 & 4.017642 & 1.309058 & $\mathrm{H}$ & -0.31751 & 3.627306 & 2.504162 \\
\hline $\mathrm{H}$ & 4.64897 & 1.362226 & 1.404621 & $\mathrm{H}$ & 5.060919 & 0.515291 & -1.58404 & $\mathrm{H}$ & 4.673472 & 1.019899 & 1.417434 \\
\hline $\mathrm{H}$ & 4.793051 & -0.40238 & 1.573763 & $\mathrm{H}$ & 4.992421 & -1.16175 & -0.99346 & $\mathrm{H}$ & 4.673803 & -0.74222 & 1.659689 \\
\hline $\mathrm{H}$ & 5.328874 & 0.42121 & 0.092089 & $\mathrm{H}$ & 5.449001 & 0.168839 & 0.089415 & $\mathrm{H}$ & 5.303648 & -0.02458 & 0.160086 \\
\hline $\mathrm{H}$ & -1.802 & -1.01725 & -1.2931 & $\mathrm{H}$ & -2.80908 & -2.56908 & -1.1894 & $\mathrm{H}$ & -1.95437 & -0.83243 & -1.27816 \\
\hline $\mathrm{H}$ & -2.57896 & -2.4961 & -0.77958 & $\mathrm{H}$ & -2.1828 & -0.95646 & -1.35124 & $\mathrm{H}$ & -2.73839 & -2.34402 & -0.87266 \\
\hline
\end{tabular}




\begin{tabular}{|c|c|c|c|c|c|c|c|c|c|c|c|}
\hline $\mathrm{H}$ & -5.02135 & -2.27755 & -0.01816 & $\mathrm{H}$ & -4.53062 & -3.03602 & 0.756961 & $\mathrm{H}$ & -5.23524 & -2.08895 & -0.29411 \\
\hline $\mathrm{H}$ & -4.2405 & -1.99859 & 1.531174 & $\mathrm{H}$ & -3.32056 & -2.57436 & 1.94471 & $\mathrm{H}$ & -4.49741 & -2.117 & 1.300296 \\
\hline $\mathrm{H}$ & -5.46343 & -0.85387 & 0.944762 & $\mathrm{H}$ & -4.87405 & -1.71975 & 1.89728 & $\mathrm{H}$ & -5.65464 & -0.83449 & 0.889016 \\
\hline $\mathrm{H}$ & 0.48716 & -0.18035 & -2.91548 & $\mathrm{H}$ & 1.460975 & 0.814111 & 2.407034 & $\mathrm{H}$ & 0.525042 & -0.40567 & -2.96631 \\
\hline
\end{tabular}

\begin{tabular}{|l|r|r|r|r|r|r|r|}
\hline $\mathrm{C}$ & -1.5125 & -1.73828 & -1.89919 & $\mathrm{C}$ & 1.84326 & -2.0705 & 1.60581 \\
\hline $\mathrm{C}$ & -2.03732 & -0.82768 & -0.82384 & $\mathrm{C}$ & 1.97797 & -0.7974 & 0.812267 \\
\hline $\mathrm{C}$ & -1.11418 & -0.86485 & 0.387039 & $\mathrm{C}$ & 1.084761 & -0.84744 & -0.42091 \\
\hline
\end{tabular}

\begin{tabular}{|l|l|l|l|l|l|l|l|}
\hline $\mathrm{C}$ & 0.122659 & -1.76613 & -0.04761 & $\mathrm{C}$ & 0.187816 & -2.14705 & -0.24608 \\
\hline $\mathrm{C}$ & 0.002803 & -1.77485 & -1.60313 & $\mathrm{C}$ & 1.156658 & -3.0227 & 0.610446 \\
\hline
\end{tabular}

\begin{tabular}{|l|l|l|l|l|l|r|r|}
\hline $\mathrm{C}$ & 0.002803 & -1.77485 & -1.60313 & $\mathrm{C}$ & 1.156658 & -3.0227 & 0.610446 \\
\hline $\mathrm{C}$ & -0.88348 & 0.606027 & 0.815912 & $\mathrm{C}$ & 0.533901 & 0.576237 & -0.66571 \\
\hline
\end{tabular}

\begin{tabular}{|l|l|l|l|l|l|l|l|}
\hline $\mathrm{C}$ & -0.16389 & 1.526514 & -0.20407 & $\mathrm{C}$ & -0.32099 & 1.260495 & 0.410246 \\
\hline $\mathrm{C}$ & 1.467602 & -1.22014 & 0.498965 & $\mathrm{C}$ & -1.13682 & -1.95002 & 0.548642 \\
\hline
\end{tabular}

\begin{tabular}{|c|r|r|r|r|r|r|r|}
\hline C & 0.965508 & 2.39593 & 0.412136 & $\mathrm{C}$ & -0.93308 & 2.610746 & -0.07803 \\
\hline $\mathrm{C}$ & 2.107997 & 2.680282 & -0.61034 & $\mathrm{C}$ & -2.45223 & 2.679796 & 0.230637 \\
\hline
\end{tabular}

\begin{tabular}{|c|c|c|c|c|c|c|c|}
\hline $\mathrm{C}$ & 2.740941 & 1.377614 & -1.08787 & $\mathrm{C}$ & -3.13834 & 1.467326 & -0.39763 \\
\hline $\mathrm{C}$ & 3.796656 & 0.76624 & -0.23443 & $\mathrm{C}$ & -3.75597 & 0.477796 & 0.532023 \\
\hline
\end{tabular}

\begin{tabular}{|l|l|l|l|l|l|l|l|}
\hline $\mathrm{C}$ & -3.06715 & 0.034884 & -0.82877 & $\mathrm{C}$ & 2.772098 & 0.271189 & 0.995049 \\
\hline & -2.18103 & 1.159805 & 1.168005 & $\mathrm{O}$ & 1.697554 & 1.425867 & -0.90202 \\
\hline
\end{tabular}

\begin{tabular}{|l|l|l|l|l|l|l|l|}
$\mathrm{O}$ & -2.18103 & 1.159805 & 1.168005 & $\mathrm{O}$ & 1.697554 & 1.425867 & -0.90202 \\
\hline $\mathrm{C}$ & -3.25704 & 0.928466 & 0.356272 & $\mathrm{C}$ & 2.745776 & 1.369426 & -0.02268 \\
\hline $\mathrm{C}$ & -0.07263 & -3.21276 & 0.458017 & $\mathrm{C}$ & -0.11486 & -2.86307 & -1.57405 \\
\hline
\end{tabular}

\begin{tabular}{|l|l|l|l|l|l|l|l|}
\hline $\mathrm{C}$ & -0.07263 & -3.21276 & 0.458017 & $\mathrm{C}$ & -0.11486 & -2.86307 & -1.57405 \\
\hline $\mathrm{O}$ & -4.3062 & 1.477969 & 0.609719 & $\mathrm{O}$ & 3.615098 & 2.209513 & -0.10267 \\
\hline
\end{tabular}

\begin{tabular}{|l|l|l|l|l|l|l|l|}
\hline $\mathrm{O}$ & 2.314296 & 0.825729 & -2.09824 & $\mathrm{O}$ & -3.08012 & 1.291111 & -1.60686 \\
\hline $\mathrm{C}$ & 0.411427 & 3.704136 & 0.993692 & $\mathrm{C}$ & -0.19428 & 3.827091 & 0.492076 \\
\hline
\end{tabular}

\begin{tabular}{|l|l|l|l|l|l|l|l|}
\hline C & -4.08826 & 0.185951 & -1.92042 & $\mathrm{C}$ & 3.755347 & 0.453065 & 2.116218 \\
\hline $\mathrm{C}$ & 2.764566 & -1.53088 & -0.29705 & $\mathrm{C}$ & -2.35475 & -1.44583 & -0.27701 \\
\hline
\end{tabular}

\begin{tabular}{|l|l|l|l|l|l|l|l|}
\hline $\mathrm{C}$ & 2.764566 & -1.53088 & -0.29705 & $\mathrm{C}$ & -2.35475 & -1.44583 & -0.27701 \\
\hline $\mathrm{C}$ & 3.852923 & -0.54182 & 0.099131 & $\mathrm{C}$ & -3.43357 & -0.82931 & 0.589194 \\
\hline
\end{tabular}

\begin{tabular}{|c|c|c|c|c|c|c|}
\hline 3.852923 & 0.07102 & ט. & 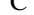 & & -0.0297 & 0. \\
\hline 4.943769 & -1.06627 & 0.992364 & $\mathrm{C}$ & -4.08689 & -1.74048 & 1.597488 \\
\hline
\end{tabular}

\begin{tabular}{|l|l|l|l|l|l|l|l|}
$\mathrm{O}$ & -1.92355 & -1.50027 & 1.402021 & $\mathrm{O}$ & 2.078232 & -1.11617 & -1.46086 \\
\hline $\mathrm{O}$ & -1.22537 & -1.43643 & 2.684771 & $\mathrm{O}$ & 1.541145 & -0.82337 & -2.78434 \\
\hline
\end{tabular}

\begin{tabular}{|l|l|l|l|l|l|l|l|}
\hline $\mathrm{O}$ & -1.22537 & -1.43643 & 2.684771 & $\mathrm{O}$ & 1.541145 & -0.82337 & -2.78434 \\
\hline $\mathrm{H}$ & -1.95041 & -2.74082 & -1.80311 & $\mathrm{H}$ & 2.803844 & -2.44851 & 1.969245 \\
\hline
\end{tabular}

\begin{tabular}{|c|c|c|c|c|c|c|}
\hline-1.95041 & -2.74082 & -1.80311 & $\mathrm{H}$ & 2.803844 & -2.44851 & 1.969245 \\
\hline-1.74203 & -1.38354 & -2.90771 & $\mathrm{H}$ & 1.215326 & -1.90294 & 2.492068 \\
\hline 0.48511 & -0.89764 & -2.04477 & $\mathrm{H}$ & 1.915797 & -3.45165 & -0.05121 \\
\hline
\end{tabular}

\begin{tabular}{|c|c|c|c|c|c|c|c|}
$\mathrm{H}$ & 0.48511 & -0.89764 & -2.04477 & $\mathrm{H}$ & 1.915797 & -3.45165 & -0.05121 \\
\hline $\mathrm{H}$ & 0.485948 & -2.65629 & -2.03575 & $\mathrm{H}$ & 0.635461 & -3.85197 & 1.097674 \\
\hline
\end{tabular}

\begin{tabular}{|c|c|c|c|c|c|c|c|}
\hline $\mathrm{H}$ & 0.485948 & -2.65629 & -2.03575 & $\mathrm{H}$ & 0.635461 & -3.85197 & 1.097674 \\
\hline $\mathrm{H}$ & -0.34832 & 0.611666 & 1.76712 & $\mathrm{H}$ & -0.00154 & 0.592573 & -1.61608 \\
\hline $\mathrm{H}$ & 0.235031 & 0.940553 & -1.03115 & $\mathrm{H}$ & -1.11504 & 0.587604 & 0.724363 \\
\hline $\mathrm{H}$ & -0.91452 & 2.186143 & -0.65124 & $\mathrm{H}$ & 0.291131 & 1.426324 & 1.302535 \\
\hline $\mathrm{H}$ & 1.585266 & -1.5641 & 1.533506 & $\mathrm{H}$ & -1.39543 & -2.92579 & 0.976745 \\
\hline $\mathrm{H}$ & 1.412779 & -0.13931 & 0.564719 & $\mathrm{H}$ & -0.9676 & -1.29383 & 1.409734 \\
\hline $\mathrm{H}$ & 1.421156 & 1.835549 & 1.240049 & $\mathrm{H}$ & -0.85198 & 2.643029 & -1.17011 \\
\hline $\mathrm{H}$ & 1.70349 & 3.203386 & -1.48323 & $\mathrm{H}$ & -2.86626 & 3.595032 & -0.20869 \\
\hline $\mathrm{H}$ & 2.854214 & 3.324752 & -0.13104 & $\mathrm{H}$ & -2.62539 & 2.714707 & 1.312795 \\
\hline $\mathrm{H}$ & 4.522258 & 1.453161 & 0.198581 & $\mathrm{H}$ & -4.4465 & 0.887067 & 1.269517 \\
\hline
\end{tabular}




\begin{tabular}{|c|c|c|c|c|c|c|c|}
\hline $\mathrm{H}$ & -0.09775 & -3.25703 & 1.547995 & $\mathrm{H}$ & -0.65978 & -3.79167 & -1.36694 \\
\hline $\mathrm{H}$ & -0.99901 & -3.66132 & 0.09073 & $\mathrm{H}$ & -0.72266 & -2.25824 & -2.2496 \\
\hline $\mathrm{H}$ & 0.762371 & -3.82782 & 0.104357 & $\mathrm{H}$ & 0.805847 & -3.11668 & -2.09935 \\
\hline $\mathrm{H}$ & 0.007354 & 4.341824 & 0.199065 & $\mathrm{H}$ & -0.61377 & 4.761193 & 0.103309 \\
\hline $\mathrm{H}$ & 1.187487 & 4.27062 & 1.519118 & $\mathrm{H}$ & 0.865851 & 3.797281 & 0.225414 \\
\hline $\mathrm{H}$ & -0.40155 & 3.502315 & 1.698367 & $\mathrm{H}$ & -0.26955 & 3.858705 & 1.585948 \\
\hline $\mathrm{H}$ & -5.09136 & 0.025339 & -1.51376 & $\mathrm{H}$ & 4.753837 & 0.646509 & 1.713785 \\
\hline $\mathrm{H}$ & -3.91849 & -0.52263 & -2.73301 & $\mathrm{H}$ & 3.792085 & -0.42428 & 2.764942 \\
\hline $\mathrm{H}$ & -4.08026 & 1.201763 & -2.33096 & $\mathrm{H}$ & 3.49481 & 1.324444 & 2.727715 \\
\hline $\mathrm{H}$ & 2.578424 & -1.4366 & -1.3668 & $\mathrm{H}$ & -2.78131 & -2.2961 & -0.82305 \\
\hline $\mathrm{H}$ & 3.102977 & -2.55434 & -0.10412 & $\mathrm{H}$ & -2.04638 & -0.73187 & -1.03952 \\
\hline $\mathrm{H}$ & 4.520788 & -1.52658 & 1.894534 & $\mathrm{H}$ & -4.55234 & -2.59576 & 1.091339 \\
\hline $\mathrm{H}$ & 5.646284 & -0.28623 & 1.296593 & $\mathrm{H}$ & -3.35438 & -2.15631 & 2.299518 \\
\hline $\mathrm{H}$ & 5.506757 & -1.85637 & 0.479089 & $\mathrm{H}$ & -4.8575 & -1.22448 & 2.175543 \\
\hline $\mathrm{H}$ & -1.78153 & -0.77531 & 3.131759 & $\mathrm{H}$ & 1.923196 & 0.063891 & -2.91177 \\
\hline
\end{tabular}

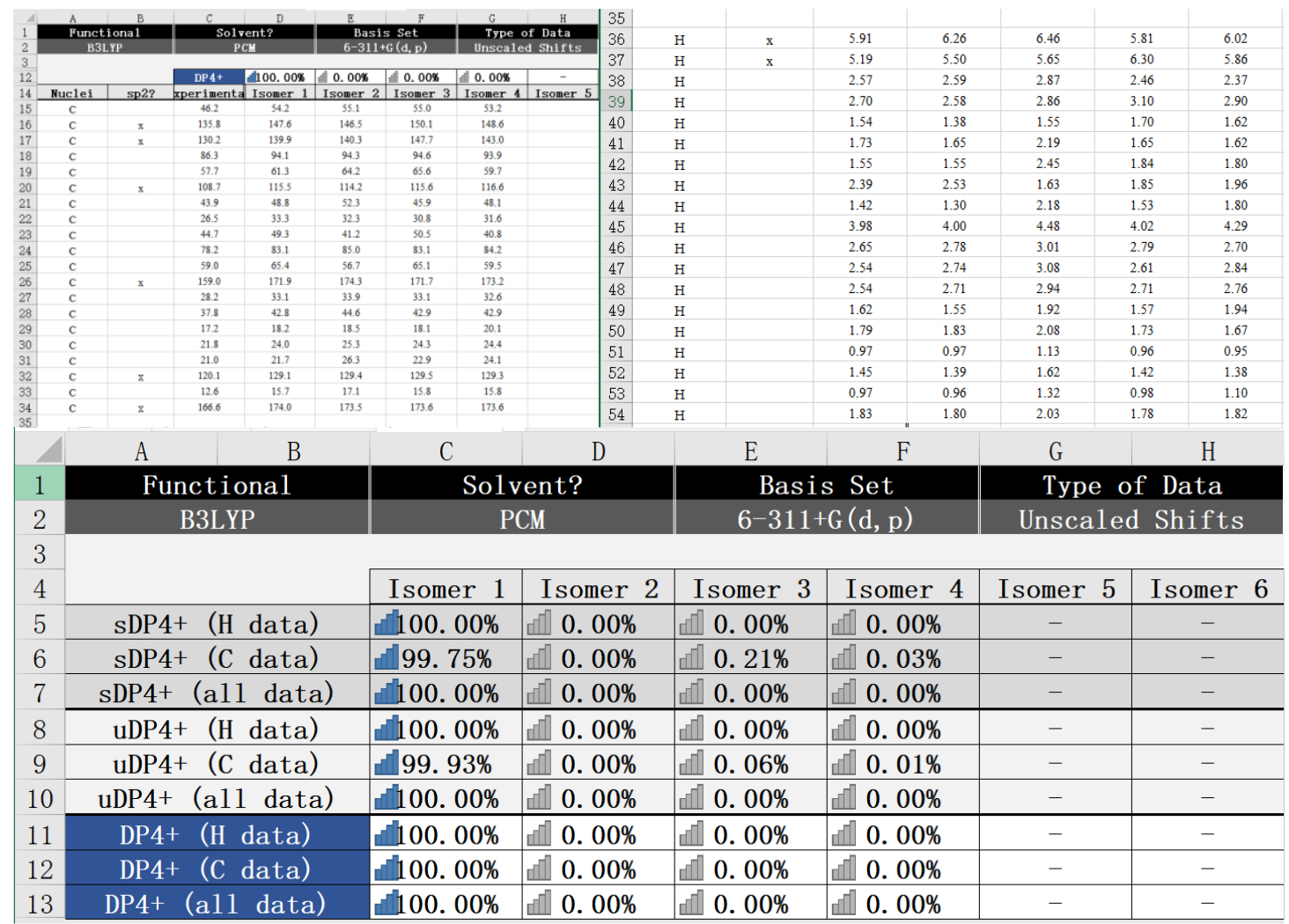

Figure S4. Detailed DP4+ probability (calculated at PCM/b3lyp/6-311+G(d,p) level) for compound 3. Isomer 1 is $(1 S, 4 S, 6 S, 8 S, 10 R, 11 S)-\mathbf{3 a}$, isomer 2 is $(1 S, 4 S, 6 S, 8 R, 10 R, 11 S)-\mathbf{3 b}$, isomer 3 is $(1 S, 4 R, 6 R, 8 S, 10 R, 11 S)-\mathbf{3 c}$, and isomer 4 is $(1 S, 4 R, 6 R, 8 R, 10 R, 11 S)-\mathbf{3 d}$. 
Table S7. Stable conformers of compound 3 with $(1 S, 4 S, 6 S, 8 S, 10 R, 11 S)-3 a,(1 S, 4 S, 6 S, 8 R$, $10 R, 11 S)-\mathbf{3 b},(1 S, 4 R, 6 R, 8 S, 10 R, 11 S)-\mathbf{3 c}$ and $(1 S, 4 R, 6 R, 8 R, 10 R, 11 S)-\mathbf{3 d}$ configurations, respectively.

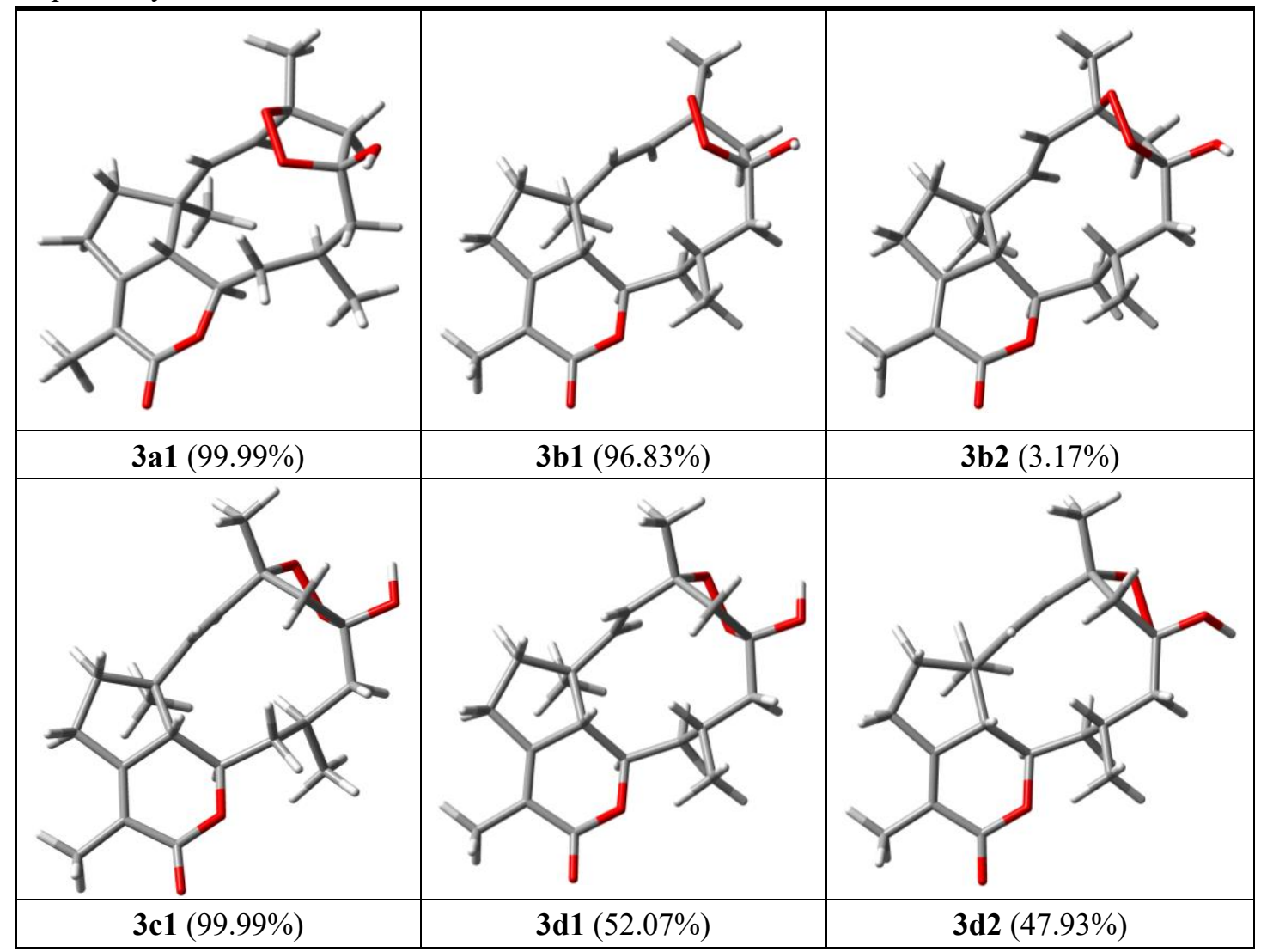

Table S8. Important thermodynamic parameters (a.u.) of the optimized compound $\mathbf{3}$ with simplified structures at B3LYP/6-31+G(d,p) level in the gas phase.

\begin{tabular}{|c|c|c|}
\hline NO. & E+ZPE & G \\
\hline 3a1 & -1154.593906 & -1154.643993 \\
\hline 3b1 & -1154.591713 & -1154.641713 \\
\hline 3b2 & -1154.587403 & -1154.638487 \\
\hline 3c1 & -1154.588354 & -1154.639008 \\
\hline 3d1 & -1154.592947 & -1154.643333 \\
\hline 3d2 & -1154.593467 & -1154.643255 \\
\hline
\end{tabular}

Table S9. Optimized Z-Matrixes of compound 3 with simplified structures in the Gas Phase $(\AA)$ at B3LYP/6-31+G(d,p) level.

\begin{tabular}{|l|r|r|r|l|r|r|r|l|r|r|r|}
\hline \multicolumn{9}{|c|}{ 3a1 } & \multicolumn{9}{|c|}{ 3b1 } & \multicolumn{4}{|c|}{ 3b2 } \\
\hline C & 3.071785 & 2.422797 & -0.23723 & C & -2.92982 & -2.29694 & -0.92344 & C & -2.81627 & -2.30853 & -0.83336 \\
\hline C & 3.011339 & 0.907812 & -0.27442 & C & -2.89093 & -0.86724 & -0.42099 & C & -2.81316 & -0.84754 & -0.4231 \\
\hline C & 1.568622 & 0.476303 & -0.3915 & C & -1.45201 & -0.44934 & -0.21003 & C & -1.41644 & -0.46077 & -0.00196 \\
\hline C & 0.785774 & 1.658899 & 0.293651 & C & -0.70512 & -1.81589 & 0.038874 & C & -0.8088 & -1.82154 & 0.50666 \\
\hline C & 1.58944 & 2.86492 & -0.27993 & C & -1.44452 & -2.71851 & -0.99244 & C & -1.36407 & -2.78202 & -0.58175 \\
\hline C & 1.346923 & -0.95354 & 0.105986 & C & -1.36268 & 0.650914 & 0.849675 & C & -1.42255 & 0.740405 & 0.941809 \\
\hline
\end{tabular}




\begin{tabular}{|c|c|c|c|c|c|c|c|c|c|c|c|}
\hline $\mathrm{C}$ & 0.029888 & -1.60702 & -0.36893 & $\mathrm{C}$ & -0.00352 & 1.34634 & 1.040608 & $\mathrm{C}$ & -0.06571 & 1.420775 & 1.202887 \\
\hline $\mathrm{C}$ & -1.7386 & 1.595423 & 0.608807 & $\mathrm{C}$ & 1.778987 & -1.70012 & 0.561612 & $\mathrm{C}$ & 1.57422 & -1.595 & -0.39526 \\
\hline $\mathrm{C}$ & -0.65157 & 1.64633 & -0.16091 & $\mathrm{C}$ & 0.757417 & -1.65216 & -0.29233 & $\mathrm{C}$ & 0.70229 & -1.74006 & 0.603192 \\
\hline $\mathrm{C}$ & -1.02609 & -1.8696 & 0.728354 & $\mathrm{C}$ & 0.663363 & 2.011739 & -0.18777 & $\mathrm{C}$ & 0.741643 & 1.94494 & -0.01193 \\
\hline $\mathrm{C}$ & -2.41167 & -2.20844 & 0.12545 & $\mathrm{C}$ & 2.174995 & 2.297144 & 0.022826 & $\mathrm{C}$ & 2.237579 & 2.194039 & 0.336772 \\
\hline $\mathrm{C}$ & -3.33185 & -1.05513 & -0.32728 & $\mathrm{C}$ & 3.217073 & 1.16398 & -0.16147 & $\mathrm{C}$ & 3.287506 & 1.10126 & 0.02323 \\
\hline $\mathrm{C}$ & -3.68796 & 0.020015 & 0.721033 & $\mathrm{C}$ & 3.428164 & 0.128095 & 0.964376 & $\mathrm{C}$ & 3.308824 & -0.20466 & 0.850698 \\
\hline $\mathrm{C}$ & -3.14172 & 1.32829 & 0.097452 & $\mathrm{C}$ & 3.188891 & -1.23414 & 0.258947 & $\mathrm{C}$ & 3.055457 & -1.2999 & -0.20825 \\
\hline $\mathrm{C}$ & 4.019818 & 0.024583 & -0.19757 & $\mathrm{C}$ & -3.92088 & -0.04768 & -0.15058 & $\mathrm{C}$ & -3.84123 & 0.01582 & -0.38106 \\
\hline $\mathrm{O}$ & 2.411089 & -1.81562 & -0.39184 & $\mathrm{O}$ & -2.34214 & 1.684893 & 0.55867 & $\mathrm{O}$ & -2.32916 & 1.761426 & 0.444934 \\
\hline $\mathrm{C}$ & 3.716405 & -1.42334 & -0.35575 & $\mathrm{C}$ & -3.63205 & 1.352938 & 0.256916 & $\mathrm{C}$ & -3.57075 & 1.433275 & -0.02017 \\
\hline $\mathrm{C}$ & 5.477002 & 0.373878 & -0.06727 & $\mathrm{C}$ & -5.37564 & -0.38776 & -0.32516 & $\mathrm{C}$ & -5.2593 & -0.29636 & -0.77347 \\
\hline $\mathrm{O}$ & 4.580965 & -2.26058 & -0.51176 & $\mathrm{O}$ & -4.47893 & 2.220868 & 0.283812 & $\mathrm{O}$ & -4.38676 & 2.317787 & -0.17109 \\
\hline $\mathrm{C}$ & 0.930117 & 1.637245 & 1.825783 & $\mathrm{C}$ & -0.948 & -2.35351 & 1.46041 & $\mathrm{C}$ & -1.37453 & -2.22976 & 1.884012 \\
\hline $\mathrm{C}$ & -4.06915 & 2.533228 & 0.265151 & $\mathrm{C}$ & 4.240407 & -2.29664 & 0.583245 & $\mathrm{C}$ & 3.890691 & -2.56902 & -0.02546 \\
\hline $\mathrm{C}$ & -0.58019 & -3.00957 & 1.660326 & $\mathrm{C}$ & -0.02909 & 3.334731 & -0.56641 & $\mathrm{C}$ & 0.138902 & 3.244925 & -0.57761 \\
\hline $\mathrm{O}$ & -4.55951 & -1.5703 & -0.79592 & $\mathrm{O}$ & 4.495202 & 1.724717 & -0.3846 & $\mathrm{O}$ & 4.584819 & 1.643915 & 0.146721 \\
\hline $\mathrm{H}$ & 1.297113 & 0.503046 & -1.45998 & $\mathrm{H}$ & -1.07325 & -0.0513 & -1.16423 & $\mathrm{H}$ & -0.85262 & -0.19752 & -0.90858 \\
\hline $\mathrm{O}$ & -3.15116 & 1.024971 & -1.31155 & $\mathrm{O}$ & 3.382756 & -0.9196 & -1.13398 & $\mathrm{O}$ & 3.564076 & -0.67943 & -1.40899 \\
\hline $\mathrm{O}$ & -2.64308 & -0.34903 & -1.36466 & $\mathrm{O}$ & 2.780643 & 0.405215 & -1.29078 & $\mathrm{O}$ & 3.025283 & 0.677888 & -1.32147 \\
\hline $\mathrm{H}$ & 3.650958 & 2.828713 & -1.07416 & $\mathrm{H}$ & -3.43133 & -2.37718 & -1.89427 & $\mathrm{H}$ & -3.12066 & -2.43943 & -1.87777 \\
\hline $\mathrm{H}$ & 3.570292 & 2.768169 & 0.676227 & $\mathrm{H}$ & -3.49815 & -2.93103 & -0.2322 & $\mathrm{H}$ & -3.53605 & -2.87561 & -0.23135 \\
\hline $\mathrm{H}$ & 1.398185 & 3.785293 & 0.279348 & $\mathrm{H}$ & -1.28976 & -3.78224 & -0.78981 & $\mathrm{H}$ & -0.77507 & -2.67937 & -1.49875 \\
\hline $\mathrm{H}$ & 1.280184 & 3.045053 & -1.31591 & $\mathrm{H}$ & -1.04653 & -2.51883 & -1.99393 & $\mathrm{H}$ & -1.30189 & -3.82945 & -0.27265 \\
\hline $\mathrm{H}$ & 1.419286 & -0.97354 & 1.201039 & $\mathrm{H}$ & -1.6489 & 0.219632 & 1.818431 & $\mathrm{H}$ & -1.82358 & 0.419941 & 1.912935 \\
\hline $\mathrm{H}$ & -0.41373 & -0.99729 & -1.15809 & $\mathrm{H}$ & -0.13853 & 2.10192 & 1.82562 & $\mathrm{H}$ & 0.536483 & 0.696668 & 1.761606 \\
\hline $\mathrm{H}$ & 0.299572 & -2.56743 & -0.82328 & $\mathrm{H}$ & 0.663637 & 0.584961 & 1.451034 & $\mathrm{H}$ & -0.25291 & 2.255044 & 1.89143 \\
\hline $\mathrm{H}$ & -1.66035 & 1.654884 & 1.692997 & $\mathrm{H}$ & 1.620865 & -1.9698 & 1.604917 & $\mathrm{H}$ & 1.261627 & -1.63384 & -1.43742 \\
\hline $\mathrm{H}$ & -0.79603 & 1.579172 & -1.23839 & $\mathrm{H}$ & 0.969747 & -1.35858 & -1.31873 & $\mathrm{H}$ & 1.083958 & -1.6988 & 1.623585 \\
\hline $\mathrm{H}$ & -1.13064 & -0.96017 & 1.332455 & $\mathrm{H}$ & 0.588433 & 1.330432 & -1.04082 & $\mathrm{H}$ & 0.722403 & 1.193624 & -0.80648 \\
\hline $\mathrm{H}$ & -2.27463 & -2.88235 & -0.73241 & $\mathrm{H}$ & 2.456087 & 3.067433 & -0.70626 & $\mathrm{H}$ & 2.566573 & 3.073902 & -0.23068 \\
\hline $\mathrm{H}$ & -3.00195 & -2.77065 & 0.857371 & $\mathrm{H}$ & 2.353489 & 2.750018 & 1.005834 & $\mathrm{H}$ & 2.359898 & 2.466255 & 1.391872 \\
\hline $\mathrm{H}$ & -3.2583 & -0.18726 & 1.703219 & $\mathrm{H}$ & 2.758167 & 0.278369 & 1.812069 & $\mathrm{H}$ & 2.576527 & -0.21041 & 1.656247 \\
\hline $\mathrm{H}$ & -4.77527 & 0.05077 & 0.813798 & $\mathrm{H}$ & 4.45745 & 0.220866 & 1.316126 & $\mathrm{H}$ & 4.305717 & -0.30525 & 1.283842 \\
\hline $\mathrm{H}$ & 6.031635 & 0.053256 & -0.95511 & $\mathrm{H}$ & -5.93212 & -0.19852 & 0.598529 & $\mathrm{H}$ & -5.5524 & 0.281905 & -1.65582 \\
\hline $\mathrm{H}$ & 5.92711 & -0.15211 & 0.78078 & $\mathrm{H}$ & -5.83017 & 0.247663 & -1.09212 & $\mathrm{H}$ & -5.95329 & -0.01154 & 0.023714 \\
\hline $\mathrm{H}$ & 5.623689 & 1.447698 & 0.068539 & $\mathrm{H}$ & -5.51429 & -1.43332 & -0.60849 & $\mathrm{H}$ & -5.39249 & -1.35828 & -0.99138 \\
\hline $\mathrm{H}$ & 0.504151 & 2.548175 & 2.258168 & $\mathrm{H}$ & -2.01666 & -2.43113 & 1.68055 & $\mathrm{H}$ & -1.04642 & -3.24419 & 2.133869 \\
\hline $\mathrm{H}$ & 1.979808 & 1.585197 & 2.127884 & $\mathrm{H}$ & -0.5049 & -1.71544 & 2.230165 & $\mathrm{H}$ & -2.46782 & -2.21532 & 1.904625 \\
\hline $\mathrm{H}$ & 0.416936 & 0.784662 & 2.278376 & $\mathrm{H}$ & -0.51047 & -3.35121 & 1.56534 & $\mathrm{H}$ & -1.02078 & -1.56761 & 2.68084 \\
\hline $\mathrm{H}$ & -3.67744 & 3.391561 & -0.28691 & $\mathrm{H}$ & 4.071987 & -3.19322 & -0.01941 & $\mathrm{H}$ & 4.954222 & -2.31942 & 0.016459 \\
\hline $\mathrm{H}$ & -4.13643 & 2.810295 & 1.32136 & $\mathrm{H}$ & 5.242725 & -1.91258 & 0.37785 & $\mathrm{H}$ & 3.722636 & -3.26078 & -0.85547 \\
\hline $\mathrm{H}$ & -5.07116 & 2.298176 & -0.10324 & $\mathrm{H}$ & 4.178521 & -2.57891 & 1.638491 & $\mathrm{H}$ & 3.60323 & -3.07342 & 0.90091 \\
\hline
\end{tabular}




\begin{tabular}{|c|c|c|c|c|c|c|c|c|c|c|c|}
\hline $\mathrm{H}$ & -0.54556 & -3.96266 & 1.119797 & $\mathrm{H}$ & 0.081034 & 4.074937 & 0.235693 & $\mathrm{H}$ & 0.204075 & 4.055506 & 0.158662 \\
\hline $\mathrm{H}$ & -1.26886 & -3.12761 & 2.503905 & $\mathrm{H}$ & 0.415816 & 3.758711 & -1.47346 & $\mathrm{H}$ & 0.685633 & 3.561487 & -1.47238 \\
\hline $\mathrm{H}$ & 0.419674 & -2.83319 & 2.069244 & $\mathrm{H}$ & -1.09697 & 3.196237 & -0.73542 & $\mathrm{H}$ & -0.91298 & 3.124258 & -0.83666 \\
\hline $\mathrm{H}$ & -4.36456 & -2.13402 & -1.55843 & $\mathrm{H}$ & 4.44355 & 2.247545 & -1.19774 & $\mathrm{H}$ & 4.654053 & 2.377189 & -0.48147 \\
\hline \multicolumn{4}{|c|}{$3 \mathrm{c} 1$} & \multicolumn{4}{|c|}{$3 d 1$} & \multicolumn{4}{|c|}{$3 \mathrm{~d} 2$} \\
\hline $\mathrm{C}$ & 3.157227 & 2.405441 & -0.00552 & $\mathrm{C}$ & -2.85026 & -2.3764 & -0.61413 & $\mathrm{C}$ & -3.04492 & -2.31501 & -0.7497 \\
\hline $\mathrm{C}$ & 3.086458 & 0.900265 & -0.17668 & $\mathrm{C}$ & -2.82353 & -0.88695 & -0.32746 & $\mathrm{C}$ & -2.96702 & -0.86069 & -0.328 \\
\hline $\mathrm{C}$ & 1.648738 & 0.495362 & -0.40859 & $\mathrm{C}$ & -1.4029 & -0.46518 & -0.03293 & $\mathrm{C}$ & -1.51613 & -0.44091 & -0.24769 \\
\hline $\mathrm{C}$ & 0.831443 & 1.64058 & 0.297565 & $\mathrm{C}$ & -0.74136 & -1.78476 & 0.515444 & $\mathrm{C}$ & -0.74919 & -1.7944 & -0.00389 \\
\hline $\mathrm{C}$ & 1.687503 & 2.871643 & -0.1262 & $\mathrm{C}$ & -1.37952 & -2.82546 & -0.4481 & $\mathrm{C}$ & -1.56929 & -2.73907 & -0.93151 \\
\hline $\mathrm{C}$ & 1.395499 & -0.96153 & -0.01626 & $\mathrm{C}$ & -1.36721 & 0.794625 & 0.831937 & $\mathrm{C}$ & -1.34224 & 0.706356 & 0.747847 \\
\hline $\mathrm{C}$ & 0.086696 & -1.57765 & -0.56087 & $\mathrm{C}$ & -0.00688 & 1.491047 & 0.999583 & $\mathrm{C}$ & 0.033424 & 1.386982 & 0.824406 \\
\hline $\mathrm{C}$ & -1.71489 & 1.574885 & 0.404829 & $\mathrm{C}$ & 1.53483 & -1.57697 & -0.61393 & $\mathrm{C}$ & 1.772299 & -1.69027 & 0.307178 \\
\hline $\mathrm{C}$ & -0.56679 & 1.675543 & -0.26608 & $\mathrm{C}$ & 0.772088 & -1.70551 & 0.471039 & $\mathrm{C}$ & 0.68177 & -1.63115 & -0.45657 \\
\hline $\mathrm{C}$ & -1.00924 & -1.86731 & 0.491089 & $\mathrm{C}$ & 0.743682 & 1.970391 & -0.26955 & $\mathrm{C}$ & 0.686839 & 1.88001 & -0.48816 \\
\hline $\mathrm{C}$ & -2.38557 & -2.18266 & -0.15432 & $\mathrm{C}$ & 2.235188 & 2.26779 & 0.026154 & $\mathrm{C}$ & 2.182064 & 2.242552 & -0.30837 \\
\hline $\mathrm{C}$ & -3.40814 & -1.03989 & -0.28842 & $\mathrm{C}$ & 3.245706 & 1.111439 & 0.046338 & $\mathrm{C}$ & 3.259556 & 1.152613 & -0.12424 \\
\hline $\mathrm{C}$ & -3.07731 & 0.171031 & -1.20923 & $\mathrm{C}$ & 3.263809 & 0.158592 & -1.18297 & $\mathrm{C}$ & 3.246665 & -0.0834 & -1.05442 \\
\hline $\mathrm{C}$ & -3.07625 & 1.370853 & -0.24123 & $\mathrm{C}$ & 3.013426 & -1.23977 & -0.57193 & $\mathrm{C}$ & 3.182647 & -1.26658 & -0.062 \\
\hline $\mathrm{C}$ & 4.082082 & 0.000744 & -0.12557 & $\mathrm{C}$ & -3.85089 & -0.02241 & -0.28912 & $\mathrm{C}$ & -3.96824 & -0.01542 & -0.03127 \\
\hline $\mathrm{O}$ & 2.466036 & -1.79378 & -0.54901 & $\mathrm{O}$ & -2.29942 & 1.779099 & 0.303725 & $\mathrm{O}$ & -2.32767 & 1.738423 & 0.456221 \\
\hline $\mathrm{C}$ & 3.773244 & -1.42393 & -0.4258 & $\mathrm{C}$ & -3.56289 & 1.419862 & -0.06545 & $\mathrm{C}$ & -3.63801 & 1.405046 & 0.267948 \\
\hline $\mathrm{C}$ & 5.533242 & 0.317248 & 0.110024 & $\mathrm{C}$ & -5.29024 & -0.36452 & -0.56025 & $\mathrm{C}$ & -5.43426 & -0.34794 & -0.07853 \\
\hline $\mathrm{O}$ & 4.635416 & -2.25431 & -0.62274 & $\mathrm{O}$ & -4.3869 & 2.291262 & -0.2488 & $\mathrm{O}$ & -4.47515 & 2.282376 & 0.298235 \\
\hline $\mathrm{C}$ & 0.850758 & 1.506868 & 1.830749 & $\mathrm{C}$ & -1.17984 & -2.09058 & 1.963631 & $\mathrm{C}$ & -0.86772 & -2.27049 & 1.454317 \\
\hline $\mathrm{C}$ & -3.67617 & 2.658727 & -0.80598 & $\mathrm{C}$ & 3.884503 & -2.35093 & -1.15977 & $\mathrm{C}$ & 4.081399 & -2.45044 & -0.42113 \\
\hline $\mathrm{C}$ & -0.58877 & -3.04064 & 1.394126 & $\mathrm{C}$ & 0.108543 & 3.23306 & -0.88204 & $\mathrm{C}$ & -0.03675 & 3.104641 & -1.07921 \\
\hline $\mathrm{O}$ & -4.61042 & -1.66155 & -0.68206 & $\mathrm{O}$ & 4.506239 & 1.713776 & 0.246402 & $\mathrm{O}$ & 4.541522 & 1.730029 & -0.26397 \\
\hline $\mathrm{H}$ & 1.446171 & 0.586969 & -1.4894 & $\mathrm{H}$ & -0.9093 & -0.24952 & -0.9924 & $\mathrm{H}$ & -1.2184 & -0.08391 & -1.24627 \\
\hline $\mathrm{O}$ & -4.00614 & 0.915391 & 0.769483 & $\mathrm{O}$ & 3.477964 & -1.05494 & 0.783641 & $\mathrm{O}$ & 3.766108 & -0.68545 & 1.131738 \\
\hline $\mathrm{O}$ & -3.54528 & -0.45358 & 1.019322 & $\mathrm{O}$ & 2.925573 & 0.254744 & 1.151563 & $\mathrm{O}$ & 3.095824 & 0.615038 & 1.19374 \\
\hline $\mathrm{H}$ & 3.805829 & 2.873268 & -0.75438 & $\mathrm{H}$ & -3.24303 & -2.59533 & -1.61337 & $\mathrm{H}$ & -3.54172 & -2.91277 & 0.024116 \\
\hline $\mathrm{H}$ & 3.58143 & 2.663471 & 0.972123 & $\mathrm{H}$ & -3.51082 & -2.89072 & 0.09413 & $\mathrm{H}$ & -3.63337 & -2.44793 & -1.66417 \\
\hline $\mathrm{H}$ & 1.462831 & 3.752851 & 0.481411 & $\mathrm{H}$ & -0.86827 & -2.79217 & -1.41599 & $\mathrm{H}$ & -1.26203 & -2.57976 & -1.9721 \\
\hline $\mathrm{H}$ & 1.460915 & 3.129694 & -1.16754 & $\mathrm{H}$ & -1.28274 & -3.84646 & -0.0676 & $\mathrm{H}$ & -1.39302 & -3.79367 & -0.70072 \\
\hline $\mathrm{H}$ & 1.439756 & -1.05527 & 1.075831 & $\mathrm{H}$ & -1.72987 & 0.535153 & 1.835509 & $\mathrm{H}$ & -1.57913 & 0.331433 & 1.752373 \\
\hline $\mathrm{H}$ & -0.30341 & -0.93356 & -1.35471 & $\mathrm{H}$ & -0.16901 & 2.356311 & 1.655315 & $\mathrm{H}$ & 0.713056 & 0.684982 & 1.312555 \\
\hline $\mathrm{H}$ & 0.360556 & -2.52241 & -1.04401 & $\mathrm{H}$ & 0.637223 & 0.80059 & 1.55112 & $\mathrm{H}$ & -0.075 & 2.239406 & 1.50754 \\
\hline $\mathrm{H}$ & -1.73595 & 1.559436 & 1.490592 & $\mathrm{H}$ & 1.106732 & -1.61341 & -1.61651 & $\mathrm{H}$ & 1.698049 & -1.99462 & 1.348439 \\
\hline $\mathrm{H}$ & -0.59953 & 1.692992 & -1.35821 & $\mathrm{H}$ & 1.272931 & -1.64505 & 1.433876 & $\mathrm{H}$ & 0.778053 & -1.33072 & -1.50101 \\
\hline $\mathrm{H}$ & -1.13801 & -0.98411 & 1.123666 & $\mathrm{H}$ & 0.695092 & 1.173683 & -1.02214 & $\mathrm{H}$ & 0.631052 & 1.074935 & -1.23084 \\
\hline $\mathrm{H}$ & -2.26122 & -2.6292 & -1.14781 & $\mathrm{H}$ & 2.332196 & 2.789931 & 0.985244 & $\mathrm{H}$ & 2.281762 & 2.93665 & 0.539215 \\
\hline $\mathrm{H}$ & -2.90015 & -2.93701 & 0.448853 & $\mathrm{H}$ & 2.619798 & 2.961094 & -0.72969 & $\mathrm{H}$ & 2.50534 & 2.80065 & -1.19355 \\
\hline
\end{tabular}




\begin{tabular}{|c|c|c|c|c|c|c|c|c|c|c|c|}
\hline $\mathrm{H}$ & -3.87333 & 0.265893 & -1.95333 & $\mathrm{H}$ & 4.249205 & 0.218795 & -1.65247 & $\mathrm{H}$ & 4.183884 & -0.09456 & -1.61322 \\
\hline $\mathrm{H}$ & -2.13526 & 0.05264 & -1.74228 & $\mathrm{H}$ & 2.51906 & 0.426916 & -1.93475 & $\mathrm{H}$ & 2.420286 & -0.07516 & -1.76469 \\
\hline $\mathrm{H}$ & 6.134386 & 0.036907 & -0.76076 & $\mathrm{H}$ & -5.64874 & 0.157152 & -1.45348 & $\mathrm{H}$ & -5.94387 & 0.266494 & -0.82751 \\
\hline $\mathrm{H}$ & 5.924772 & -0.26321 & 0.951834 & $\mathrm{H}$ & -5.92929 & -0.03334 & 0.264739 & $\mathrm{H}$ & -5.91451 & -0.12357 & 0.879631 \\
\hline $\mathrm{H}$ & 5.686673 & 1.378738 & 0.316485 & $\mathrm{H}$ & -5.43004 & -1.43855 & -0.70123 & $\mathrm{H}$ & -5.60151 & -1.40128 & -0.314 \\
\hline $\mathrm{H}$ & 0.399756 & 2.39001 & 2.293852 & $\mathrm{H}$ & -0.82002 & -3.08186 & 2.258357 & $\mathrm{H}$ & -0.41813 & -3.26207 & 1.565951 \\
\hline $\mathrm{H}$ & 1.872825 & 1.417766 & 2.2092 & $\mathrm{H}$ & -2.26737 & -2.07878 & 2.080359 & $\mathrm{H}$ & -1.91388 & -2.34138 & 1.76557 \\
\hline $\mathrm{H}$ & 0.295629 & 0.631419 & 2.177321 & $\mathrm{H}$ & -0.76137 & -1.3692 & 2.672101 & $\mathrm{H}$ & -0.36387 & -1.59743 & 2.152946 \\
\hline $\mathrm{H}$ & -3.04601 & 3.038038 & -1.61474 & $\mathrm{H}$ & 4.942053 & -2.0747 & -1.12273 & $\mathrm{H}$ & 3.737227 & -2.90834 & -1.35245 \\
\hline $\mathrm{H}$ & -3.73251 & 3.42659 & -0.02986 & $\mathrm{H}$ & 3.607048 & -2.53146 & -2.2024 & $\mathrm{H}$ & 4.048128 & -3.21028 & 0.364662 \\
\hline $\mathrm{H}$ & -4.68168 & 2.476634 & -1.19596 & $\mathrm{H}$ & 3.739864 & -3.28051 & -0.60316 & $\mathrm{H}$ & 5.115398 & -2.11769 & -0.54572 \\
\hline $\mathrm{H}$ & -0.54134 & -3.97498 & 0.822555 & $\mathrm{H}$ & 0.625454 & 3.516436 & -1.80567 & $\mathrm{H}$ & 0.417323 & 3.403426 & -2.03052 \\
\hline $\mathrm{H}$ & -1.30477 & -3.18101 & 2.210035 & $\mathrm{H}$ & -0.94906 & 3.08892 & -1.1024 & $\mathrm{H}$ & 0.027959 & 3.959154 & -0.39544 \\
\hline $\mathrm{H}$ & 0.398025 & -2.883 & 1.84114 & $\mathrm{H}$ & 0.18642 & 4.077421 & -0.18677 & $\mathrm{H}$ & -1.09603 & 2.906261 & -1.24554 \\
\hline $\mathrm{H}$ & -5.31189 & -1.00055 & -0.58767 & $\mathrm{H}$ & 5.120698 & 1.002891 & 0.480809 & $\mathrm{H}$ & 4.634546 & 2.393709 & 0.434831 \\
\hline
\end{tabular}

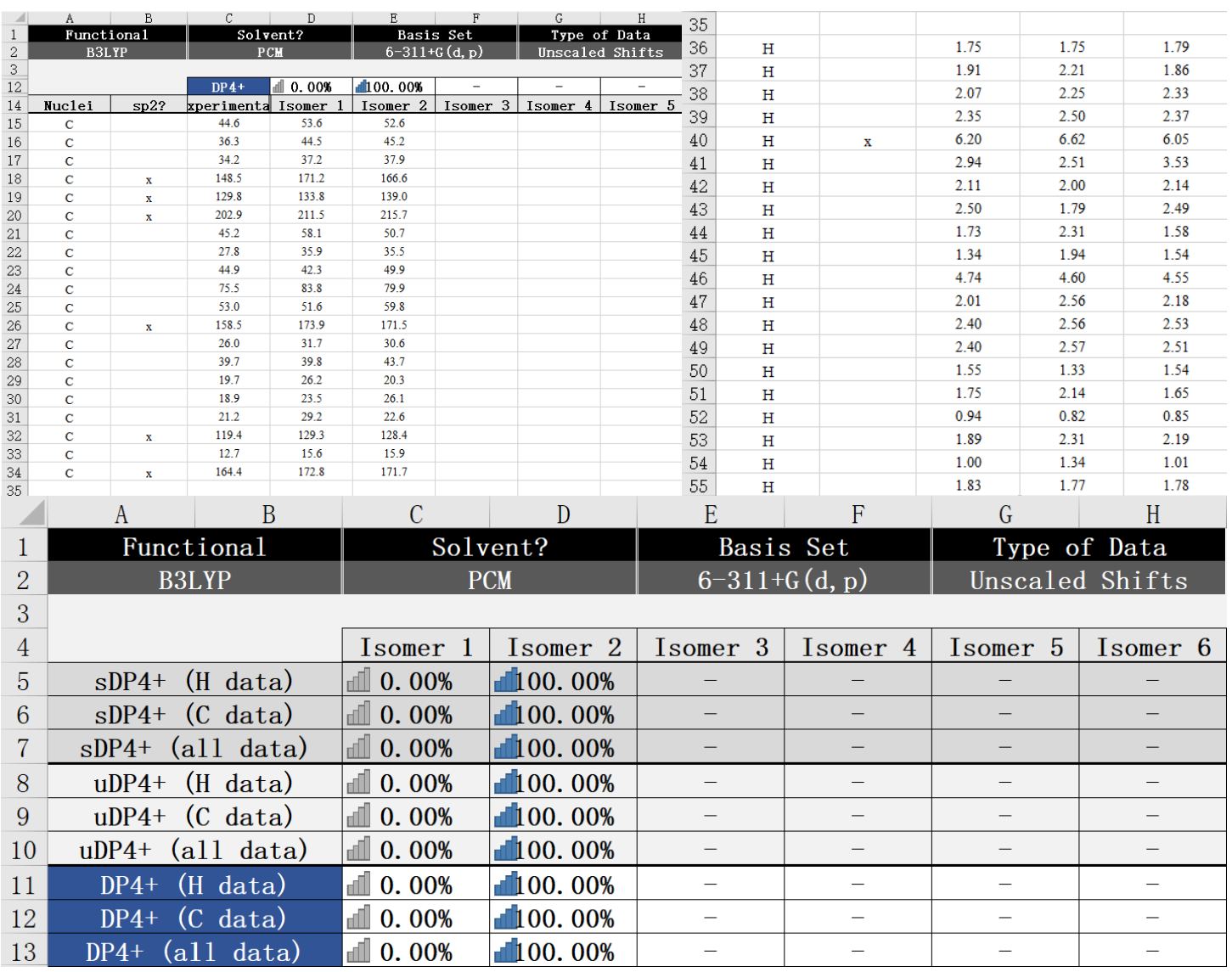

Figure S5. Detailed DP4+ probability (calculated at PCM/b3lyp/6-311+G(d,p) level) for compound 4. Isomer 1 is $(1 R, 8 R, 10 R, 11 S)-\mathbf{4 a}$, isomer 2 is $(1 R, 8 S, 10 R, 11 S)-\mathbf{4 b}$. 


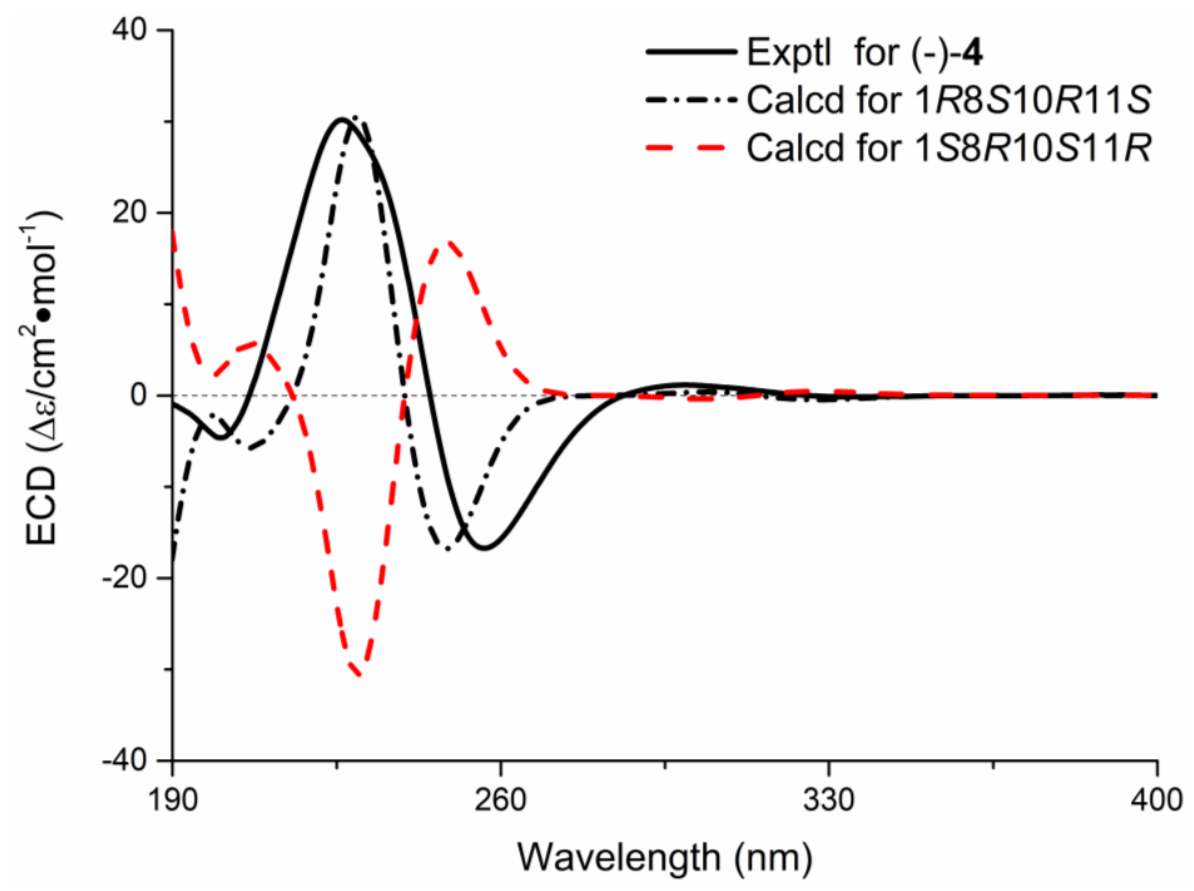

Figure S6. Experimental and calculated ECD spectra of 4

Table S10. Stable conformers of compound 4 with $(1 R, 8 R, 10 R, 11 S)$-4a and $(1 R, 8 S, 10 R, 11 S)$ 4b configurations, respectively.

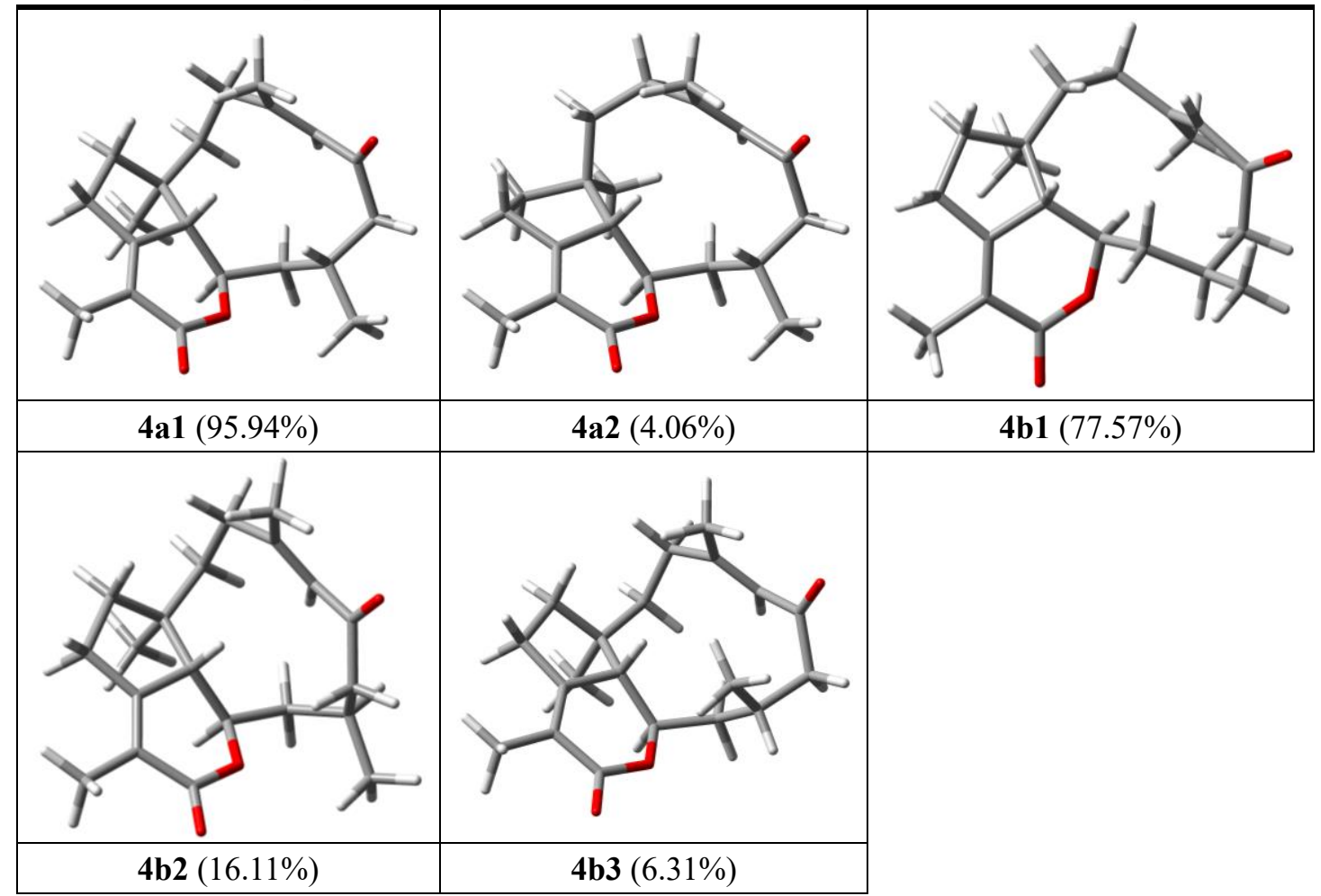

Table S11. Important thermodynamic parameters (a.u.) of the optimized compound 4 with simplified structures at $\mathrm{B} 3 \mathrm{LYP} / 6-31+\mathrm{G}(\mathrm{d}, \mathrm{p})$ level in the gas phase.

\begin{tabular}{|c|c|c|}
\hline NO. & E+ZPE & G \\
\hline 4a1 & -1004.239105 & -1004.289356 \\
\hline
\end{tabular}




\begin{tabular}{|l|l|l|}
\hline $\mathbf{4 a 2}$ & -1004.236304 & -1004.286373 \\
\hline $\mathbf{4 b 1}$ & -1154.591713 & -1154.641713 \\
\hline $\mathbf{4 b 2}$ & -1154.587403 & -1154.638487 \\
\hline $\mathbf{4 b 3}$ & -1154.588354 & -1154.639008 \\
\hline
\end{tabular}

Table S12. Optimized Z-Matrixes of compound 4 with simplified structures in the Gas Phase $(\AA)$ at B3LYP/6-31+G(d,p) level.

\begin{tabular}{|c|c|c|c|c|c|c|c|c|c|c|c|}
\hline \multicolumn{4}{|c|}{$4 a 1$} & \multicolumn{4}{|c|}{$4 a 2$} & \multicolumn{4}{|c|}{$4 \mathrm{~b} 1$} \\
\hline $\mathrm{C}$ & -2.5744 & -1.97493 & -0.69636 & $\mathrm{C}$ & -2.79349 & -1.91749 & -0.35855 & $\mathrm{C}$ & 3.297695 & 1.2098 & -1.17157 \\
\hline $\mathrm{C}$ & -2.1798 & -0.5333 & -0.45388 & $\mathrm{C}$ & -2.26526 & -0.50906 & -0.391 & $\mathrm{C}$ & 2.524844 & 0.048961 & -0.58132 \\
\hline $\mathrm{C}$ & -0.79101 & -0.46522 & 0.171637 & $\mathrm{C}$ & -0.83782 & -0.47546 & 0.168341 & $\mathrm{C}$ & 1.06334 & 0.41224 & -0.59717 \\
\hline $\mathrm{C}$ & -0.67643 & -1.88048 & 0.879143 & $\mathrm{C}$ & -0.65727 & -1.90844 & 0.862509 & $\mathrm{C}$ & 1.08465 & 1.946583 & -0.25195 \\
\hline $\mathrm{C}$ & -1.38189 & -2.78767 & -0.16599 & $\mathrm{C}$ & -2.12048 & -2.44605 & 0.914092 & $\mathrm{C}$ & 2.285635 & 2.385998 & -1.15126 \\
\hline $\mathrm{C}$ & -0.67691 & 0.818629 & 1.01878 & $\mathrm{C}$ & -0.69966 & 0.803886 & 1.024467 & $\mathrm{C}$ & 0.154541 & -0.58073 & 0.159174 \\
\hline $\mathrm{C}$ & 0.731929 & 1.271435 & 1.459731 & $\mathrm{C}$ & 0.715566 & 1.221406 & 1.489824 & $\mathrm{C}$ & -0.70779 & -1.41122 & -0.80568 \\
\hline $\mathrm{C}$ & 1.736275 & -2.81928 & 0.127206 & $\mathrm{C}$ & 1.687536 & -2.86473 & -0.0902 & $\mathrm{C}$ & -1.3505 & 2.982541 & 0.279107 \\
\hline $\mathrm{C}$ & 0.731088 & -2.43278 & 1.239701 & $\mathrm{C}$ & 0.138315 & -2.89905 & -0.05003 & $\mathrm{C}$ & -0.16457 & 2.767791 & -0.69229 \\
\hline $\mathrm{C}$ & 1.524739 & 2.133275 & 0.430759 & $\mathrm{C}$ & 1.531517 & 2.11051 & 0.505306 & $\mathrm{C}$ & -1.83002 & -2.25105 & -0.16218 \\
\hline $\mathrm{C}$ & 3.054469 & 1.821476 & 0.405188 & $\mathrm{C}$ & 3.053808 & 1.764112 & 0.497558 & $\mathrm{C}$ & -2.88561 & -1.3878 & 0.603712 \\
\hline $\mathrm{C}$ & 3.359424 & 0.643322 & -0.51361 & $\mathrm{C}$ & 3.344539 & 0.620272 & -0.4666 & $\mathrm{C}$ & -3.46531 & -0.2836 & -0.27773 \\
\hline $\mathrm{C}$ & 2.962931 & -0.67781 & 0.02635 & $\mathrm{C}$ & 2.907204 & -0.71977 & -0.00765 & $\mathrm{C}$ & -2.7468 & 1.024059 & -0.36307 \\
\hline $\mathrm{C}$ & 2.348231 & -1.66637 & -0.65309 & $\mathrm{C}$ & 2.30442 & -1.66018 & -0.76362 & $\mathrm{C}$ & -2.21068 & 1.772567 & 0.619018 \\
\hline $\mathrm{C}$ & -2.94641 & 0.547804 & -0.67489 & $\mathrm{C}$ & -2.96109 & 0.603104 & -0.68363 & $\mathrm{C}$ & 3.014919 & -1.06472 & -0.01273 \\
\hline $\mathrm{O}$ & -1.22553 & 1.959757 & 0.306236 & $\mathrm{O}$ & -1.19068 & 1.955342 & 0.28687 & $\mathrm{O}$ & 0.850842 & -1.50231 & 1.035069 \\
\hline $\mathrm{C}$ & -2.39861 & 1.891867 & -0.37922 & $\mathrm{C}$ & -2.31959 & 1.921997 & -0.47067 & $\mathrm{C}$ & 2.120279 & -1.93327 & 0.793557 \\
\hline $\mathrm{C}$ & -4.32514 & 0.528724 & -1.2775 & $\mathrm{C}$ & -4.35902 & 0.659867 & -1.23965 & $\mathrm{C}$ & 4.463874 & -1.47277 & -0.01934 \\
\hline $\mathrm{O}$ & -2.92265 & 2.923189 & -0.74426 & $\mathrm{O}$ & -2.7471 & 2.962169 & -0.92447 & $\mathrm{O}$ & 2.507085 & -2.94313 & 1.344435 \\
\hline $\mathrm{C}$ & -1.49786 & -1.88666 & 2.189631 & $\mathrm{C}$ & -0.08303 & -1.91768 & 2.288238 & $\mathrm{C}$ & 1.43371 & 2.183555 & 1.227244 \\
\hline $\mathrm{C}$ & 1.312554 & 3.631975 & 0.703869 & $\mathrm{C}$ & 1.341004 & 3.601301 & 0.828881 & $\mathrm{C}$ & -2.49837 & -3.1437 & -1.21685 \\
\hline $\mathrm{O}$ & 3.787668 & 0.826902 & -1.64623 & $\mathrm{O}$ & 3.803392 & 0.842849 & -1.58031 & $\mathrm{O}$ & -4.39863 & -0.51512 & -1.03491 \\
\hline $\mathrm{H}$ & -0.04651 & -0.42557 & -0.63628 & $\mathrm{H}$ & -0.12644 & -0.38986 & -0.66257 & $\mathrm{H}$ & 0.760146 & 0.372285 & -1.65596 \\
\hline $\mathrm{C}$ & 2.201137 & -1.70644 & -2.1488 & $\mathrm{C}$ & 2.17608 & -1.5914 & -2.26181 & $\mathrm{C}$ & -2.46695 & 1.565145 & 2.091641 \\
\hline $\mathrm{H}$ & -3.49857 & -2.20945 & -0.15485 & $\mathrm{H}$ & -3.88373 & -1.96737 & -0.32392 & $\mathrm{H}$ & 3.642739 & 0.995746 & -2.19045 \\
\hline $\mathrm{H}$ & -2.78004 & -2.17453 & -1.75399 & $\mathrm{H}$ & -2.4689 & -2.49008 & -1.23784 & $\mathrm{H}$ & 4.193331 & 1.421023 & -0.57924 \\
\hline $\mathrm{H}$ & -1.67399 & -3.75107 & 0.264449 & $\mathrm{H}$ & -2.63465 & -2.0246 & 1.787001 & $\mathrm{H}$ & 2.73171 & 3.317899 & -0.79001 \\
\hline $\mathrm{H}$ & -0.69679 & -3.00097 & -0.99197 & $\mathrm{H}$ & -2.15637 & -3.53571 & 1.011294 & $\mathrm{H}$ & 1.918705 & 2.582215 & -2.16519 \\
\hline $\mathrm{H}$ & -1.29638 & 0.68369 & 1.913509 & $\mathrm{H}$ & -1.34541 & 0.691447 & 1.905979 & $\mathrm{H}$ & -0.49036 & -0.0337 & 0.84373 \\
\hline $\mathrm{H}$ & 1.291731 & 0.379446 & 1.745024 & $\mathrm{H}$ & 0.60852 & 1.773571 & 2.431309 & $\mathrm{H}$ & -1.14098 & -0.73578 & -1.5531 \\
\hline $\mathrm{H}$ & 0.620337 & 1.862208 & 2.376808 & $\mathrm{H}$ & 1.267726 & 0.316225 & 1.735669 & $\mathrm{H}$ & -0.03432 & -2.08009 & -1.35809 \\
\hline $\mathrm{H}$ & 1.277828 & -3.53134 & -0.56604 & $\mathrm{H}$ & 1.999013 & -3.76679 & -0.63508 & $\mathrm{H}$ & -0.97884 & 3.428905 & 1.207256 \\
\hline $\mathrm{H}$ & 2.548854 & -3.372 & 0.617828 & $\mathrm{H}$ & 2.084755 & -2.96581 & 0.924644 & $\mathrm{H}$ & -1.99458 & 3.75005 & -0.17153 \\
\hline $\mathrm{H}$ & 0.551272 & -3.34762 & 1.819916 & $\mathrm{H}$ & -0.12569 & -3.90982 & 0.284865 & $\mathrm{H}$ & 0.198126 & 3.776186 & -0.93262 \\
\hline $\mathrm{H}$ & 1.219646 & -1.74654 & 1.93985 & $\mathrm{H}$ & -0.24161 & -2.82199 & -1.07517 & $\mathrm{H}$ & -0.53562 & 2.35976 & -1.64112 \\
\hline
\end{tabular}




\begin{tabular}{|c|c|c|c|c|c|c|c|c|c|c|c|}
\hline $\mathrm{H}$ & 1.134789 & 1.928166 & -0.57326 & $\mathrm{H}$ & 1.155772 & 1.94915 & -0.5116 & $\mathrm{H}$ & -1.36517 & -2.8961 & 0.592796 \\
\hline $\mathrm{H}$ & 3.417644 & 1.637212 & 1.424808 & $\mathrm{H}$ & 3.39078 & 1.526298 & 1.515108 & $\mathrm{H}$ & -2.41861 & -0.97853 & 1.501383 \\
\hline $\mathrm{H}$ & 3.591899 & 2.684015 & 0.002646 & $\mathrm{H}$ & 3.621934 & 2.628039 & 0.143132 & $\mathrm{H}$ & -3.7075 & -2.04014 & 0.914533 \\
\hline $\mathrm{H}$ & 3.013792 & -0.76099 & 1.109851 & $\mathrm{H}$ & 2.946978 & -0.87957 & 1.067847 & $\mathrm{H}$ & -2.644 & 1.367534 & -1.39228 \\
\hline $\mathrm{H}$ & -4.68146 & -0.48887 & -1.44876 & $\mathrm{H}$ & -4.75818 & -0.33173 & -1.45762 & $\mathrm{H}$ & 4.570389 & -2.48242 & -0.42845 \\
\hline $\mathrm{H}$ & -5.03913 & 1.045576 & -0.62806 & $\mathrm{H}$ & -5.03822 & 1.166515 & -0.54494 & $\mathrm{H}$ & 5.073829 & -0.7915 & -0.61602 \\
\hline $\mathrm{H}$ & -4.33303 & 1.067946 & -2.2303 & $\mathrm{H}$ & -4.3665 & 1.250844 & -2.16027 & $\mathrm{H}$ & 4.866094 & -1.5123 & 0.9983 \\
\hline $\mathrm{H}$ & -1.62098 & -2.91216 & 2.553014 & $\mathrm{H}$ & -0.14807 & -2.93233 & 2.698573 & $\mathrm{H}$ & 0.68089 & 1.766328 & 1.902631 \\
\hline $\mathrm{H}$ & -2.49608 & -1.45904 & 2.056169 & $\mathrm{H}$ & -0.65061 & -1.26333 & 2.956757 & $\mathrm{H}$ & 2.390209 & 1.72131 & 1.487888 \\
\hline $\mathrm{H}$ & -0.99911 & -1.31907 & 2.982274 & $\mathrm{H}$ & 0.964935 & -1.61796 & 2.338389 & $\mathrm{H}$ & 1.513143 & 3.255492 & 1.439157 \\
\hline $\mathrm{H}$ & 0.248592 & 3.875011 & 0.717488 & $\mathrm{H}$ & 0.281328 & 3.86347 & 0.824827 & $\mathrm{H}$ & -3.01947 & -2.54834 & -1.97277 \\
\hline $\mathrm{H}$ & 1.74669 & 3.912717 & 1.671732 & $\mathrm{H}$ & 1.85287 & 4.228997 & 0.092451 & $\mathrm{H}$ & -1.75476 & -3.7688 & -1.72277 \\
\hline $\mathrm{H}$ & 1.795208 & 4.240664 & -0.06744 & $\mathrm{H}$ & 1.755548 & 3.838457 & 1.8168 & $\mathrm{H}$ & -3.23741 & -3.80664 & -0.75617 \\
\hline $\mathrm{H}$ & 1.152481 & -1.85893 & -2.43464 & $\mathrm{H}$ & 2.850178 & -2.3317 & -2.71285 & $\mathrm{H}$ & -1.54756 & 1.313939 & 2.636367 \\
\hline $\mathrm{H}$ & 2.753258 & -2.57118 & -2.54109 & $\mathrm{H}$ & 2.455929 & -0.61574 & -2.65596 & $\mathrm{H}$ & -3.21622 & 0.801317 & 2.299626 \\
\hline $\mathrm{H}$ & 2.596239 & -0.81137 & -2.62656 & $\mathrm{H}$ & 1.163514 & -1.85431 & -2.5884 & $\mathrm{H}$ & -2.82955 & 2.506476 & 2.523448 \\
\hline
\end{tabular}

\begin{tabular}{|c|c|c|c|c|c|c|c|}
\hline \multicolumn{4}{|c|}{$4 b 2$} & \multicolumn{4}{|c|}{$4 b 3$} \\
\hline $\mathrm{C}$ & -2.21532 & -2.31742 & -0.62689 & $\mathrm{C}$ & 2.448976 & 1.955455 & -0.97052 \\
\hline $\mathrm{C}$ & -1.98802 & -0.82975 & -0.46926 & $\mathrm{C}$ & 2.202113 & 0.532198 & -0.51305 \\
\hline $\mathrm{C}$ & -0.65458 & -0.56889 & 0.223148 & $\mathrm{C}$ & 0.828432 & 0.431272 & 0.129982 \\
\hline $\mathrm{C}$ & -0.3633 & -1.93819 & 0.963161 & $\mathrm{C}$ & 0.628473 & 1.900394 & 0.696074 \\
\hline $\mathrm{C}$ & -0.93143 & -2.95292 & -0.06863 & $\mathrm{C}$ & 1.211229 & 2.730841 & -0.48053 \\
\hline $\mathrm{C}$ & -0.80558 & 0.73727 & 1.028842 & $\mathrm{C}$ & 0.78024 & -0.75928 & 1.108715 \\
\hline $\mathrm{C}$ & 0.402505 & 1.338613 & 1.781209 & $\mathrm{C}$ & -0.61081 & -1.26996 & 1.513824 \\
\hline $\mathrm{C}$ & 2.163848 & -2.55835 & 0.250847 & $\mathrm{C}$ & -1.90189 & 2.595631 & 0.025067 \\
\hline $\mathrm{C}$ & 1.105785 & -2.29402 & 1.346046 & $\mathrm{C}$ & -0.79716 & 2.384477 & 1.089417 \\
\hline $\mathrm{C}$ & 1.34605 & 2.395809 & 1.110988 & $\mathrm{C}$ & -1.40303 & -2.23906 & 0.572513 \\
\hline $\mathrm{C}$ & 1.748596 & 2.226976 & -0.37735 & $\mathrm{C}$ & -2.94527 & -2.07024 & 0.778159 \\
\hline $\mathrm{C}$ & 2.726145 & 1.132587 & -0.81614 & $\mathrm{C}$ & -3.51798 & -0.94259 & -0.08268 \\
\hline $\mathrm{C}$ & 2.631184 & -0.14533 & -0.07679 & $\mathrm{C}$ & -3.07364 & 0.423872 & 0.297795 \\
\hline $\mathrm{C}$ & 2.609806 & -1.38357 & -0.60513 & $\mathrm{C}$ & -2.57872 & 1.354816 & -0.54126 \\
\hline $\mathrm{C}$ & -2.82949 & 0.15761 & -0.82401 & $\mathrm{C}$ & 3.073345 & -0.48897 & -0.56916 \\
\hline $\mathrm{O}$ & -1.30431 & 1.784621 & 0.154482 & $\mathrm{O}$ & 1.498081 & -1.9241 & 0.620099 \\
\hline $\mathrm{C}$ & -2.40739 & 1.564683 & -0.62025 & $\mathrm{C}$ & 2.672245 & -1.8225 & -0.05862 \\
\hline $\mathrm{C}$ & -4.15714 & -0.03 & -1.50656 & $\mathrm{C}$ & 4.446831 & -0.42269 & -1.18104 \\
\hline $\mathrm{O}$ & -2.96062 & 2.514122 & -1.13239 & $\mathrm{O}$ & 3.324837 & -2.82771 & -0.24898 \\
\hline $\mathrm{C}$ & -1.19126 & -2.02493 & 2.266468 & $\mathrm{C}$ & 1.511679 & 2.113022 & 1.949148 \\
\hline $\mathrm{C}$ & 0.759233 & 3.811675 & 1.289154 & $\mathrm{C}$ & -1.06719 & -2.23334 & -0.92962 \\
\hline $\mathrm{O}$ & 3.440227 & 1.313928 & -1.794 & $\mathrm{O}$ & -4.19824 & -1.18905 & -1.06917 \\
\hline $\mathrm{H}$ & 0.111437 & -0.4299 & -0.54834 & $\mathrm{H}$ & 0.086251 & 0.284017 & -0.66563 \\
\hline $\mathrm{C}$ & 2.972207 & -1.70469 & -2.02855 & $\mathrm{C}$ & -2.64304 & 1.263895 & -2.04158 \\
\hline $\mathrm{H}$ & -2.40652 & -2.60158 & -1.6674 & $\mathrm{H}$ & 3.373529 & 2.34239 & -0.52688 \\
\hline $\mathrm{H}$ & -3.10034 & -2.62617 & -0.05672 & $\mathrm{H}$ & 2.578839 & 2.021975 & -2.05675 \\
\hline
\end{tabular}




\begin{tabular}{|c|c|c|c|c|c|c|c|}
\hline $\mathrm{H}$ & -1.09714 & -3.93866 & 0.378313 & $\mathrm{H}$ & 1.439647 & 3.758518 & -0.18 \\
\hline $\mathrm{H}$ & -0.21709 & -3.08657 & -0.88718 & $\mathrm{H}$ & 0.478214 & 2.789424 & -1.2913 \\
\hline $\mathrm{H}$ & -1.58915 & 0.552908 & 1.774822 & $\mathrm{H}$ & 1.299788 & -0.45059 & 2.02422 \\
\hline $\mathrm{H}$ & -0.00461 & 1.839566 & 2.666623 & $\mathrm{H}$ & -1.20656 & -0.38373 & 1.731085 \\
\hline $\mathrm{H}$ & 0.98287 & 0.503968 & 2.181692 & $\mathrm{H}$ & -0.49747 & -1.79181 & 2.4709 \\
\hline $\mathrm{H}$ & 1.819332 & -3.36047 & -0.41133 & $\mathrm{H}$ & -1.51676 & 3.205652 & -0.79806 \\
\hline $\mathrm{H}$ & 3.05162 & -2.97622 & 0.749051 & $\mathrm{H}$ & -2.6772 & 3.212419 & 0.500234 \\
\hline $\mathrm{H}$ & 1.486576 & -1.55241 & 2.056431 & $\mathrm{H}$ & -0.6472 & 3.363926 & 1.562442 \\
\hline $\mathrm{H}$ & 1.040533 & -3.22179 & 1.929821 & $\mathrm{H}$ & -1.18483 & 1.746124 & 1.890764 \\
\hline $\mathrm{H}$ & 2.270798 & 2.362783 & 1.705524 & $\mathrm{H}$ & -1.16669 & -3.24939 & 0.923432 \\
\hline $\mathrm{H}$ & 0.840605 & 2.082319 & -0.97717 & $\mathrm{H}$ & -3.16105 & -1.89253 & 1.839993 \\
\hline $\mathrm{H}$ & 2.186298 & 3.165526 & -0.7321 & $\mathrm{H}$ & -3.45674 & -2.98756 & 0.474159 \\
\hline $\mathrm{H}$ & 2.34753 & -0.04125 & 0.960749 & $\mathrm{H}$ & -2.97894 & 0.594149 & 1.368096 \\
\hline $\mathrm{H}$ & -4.39885 & -1.08605 & -1.64175 & $\mathrm{H}$ & 4.704399 & 0.590836 & -1.49461 \\
\hline $\mathrm{H}$ & -4.15597 & 0.459968 & -2.48536 & $\mathrm{H}$ & 5.20368 & -0.77566 & -0.47346 \\
\hline $\mathrm{H}$ & -4.96085 & 0.439777 & -0.92963 & $\mathrm{H}$ & 4.510777 & -1.08394 & -2.05143 \\
\hline $\mathrm{H}$ & -0.79536 & -1.36044 & 3.041749 & $\mathrm{H}$ & 1.584774 & 3.180454 & 2.181993 \\
\hline $\mathrm{H}$ & -1.16474 & -3.044 & 2.666521 & $\mathrm{H}$ & 1.089526 & 1.618506 & 2.830213 \\
\hline $\mathrm{H}$ & -2.24102 & -1.75814 & 2.110039 & $\mathrm{H}$ & 2.527533 & 1.730919 & 1.812661 \\
\hline $\mathrm{H}$ & 0.528482 & 4.010164 & 2.341436 & $\mathrm{H}$ & -1.22384 & -1.25809 & -1.39796 \\
\hline $\mathrm{H}$ & -0.16157 & 3.927591 & 0.712655 & $\mathrm{H}$ & -0.03527 & -2.53735 & -1.10168 \\
\hline $\mathrm{H}$ & 1.473721 & 4.571978 & 0.957252 & $\mathrm{H}$ & -1.72283 & -2.93945 & -1.44949 \\
\hline $\mathrm{H}$ & 3.26605 & -0.81609 & -2.58514 & $\mathrm{H}$ & -1.64304 & 1.371561 & -2.48174 \\
\hline $\mathrm{H}$ & 2.140444 & -2.21033 & -2.53618 & $\mathrm{H}$ & -3.23825 & 2.102152 & -2.42785 \\
\hline $\mathrm{H}$ & 3.81338 & -2.41079 & -2.04104 & $\mathrm{H}$ & -3.10673 & 0.340462 & -2.38477 \\
\hline
\end{tabular}




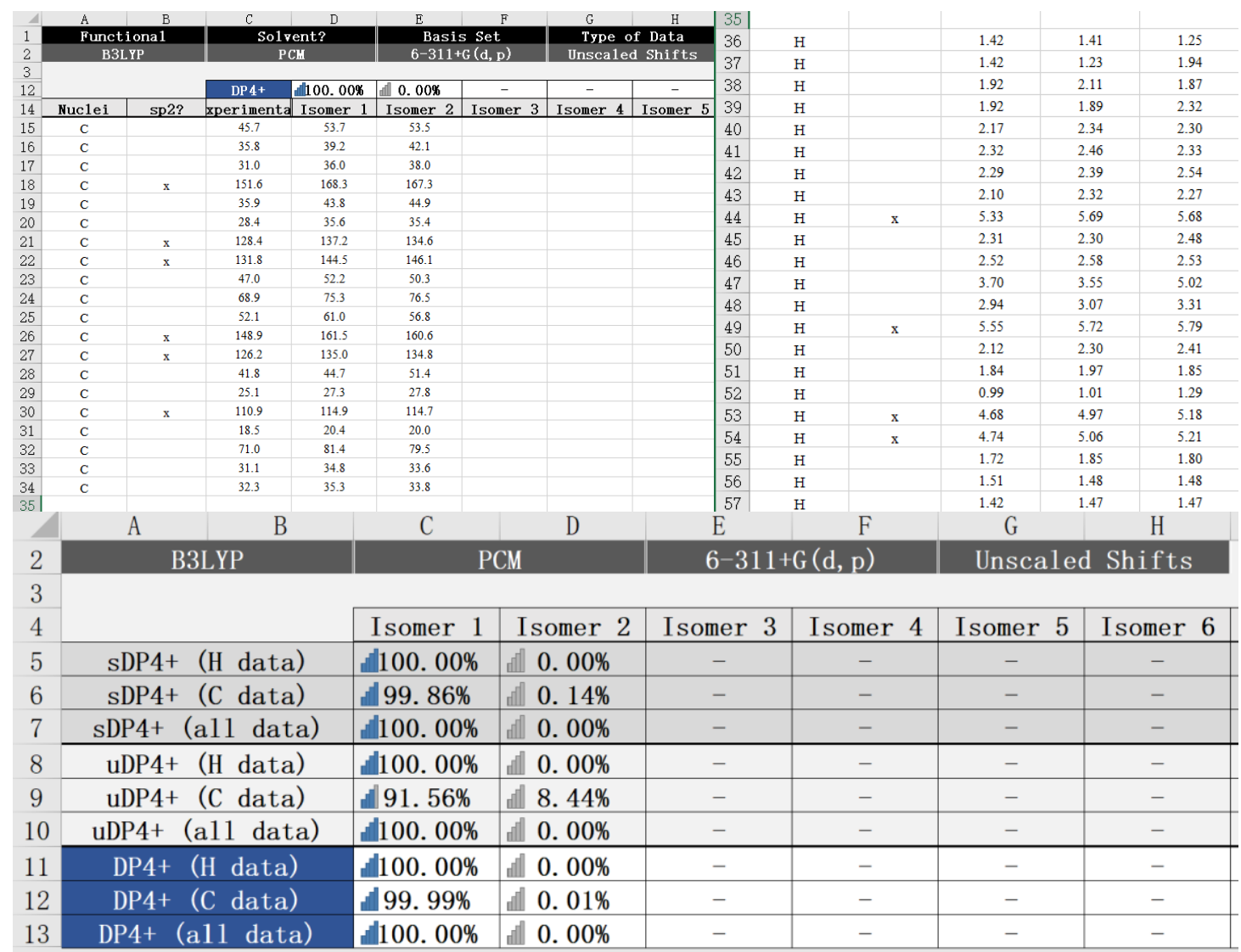

Figure S7. Detailed DP4+ probability (calculated at $\mathrm{PCM} / \mathrm{b} 31 \mathrm{yp} / 6-311+\mathrm{G}(\mathrm{d}, \mathrm{p})$ level) for compound 5. Isomer 1 is $(1 R, 10 R, 11 S)-\mathbf{5 a}$, isomer 2 is $(1 R, 10 S, 11 S)-\mathbf{5 b}$.

Table S13. Stable conformers of compound $\mathbf{3}$ with $(1 R, 10 R, 11 S)-\mathbf{5 a}$ and $(1 R, 10 S, 11 S)-\mathbf{5 b}$ configurations, respectively.

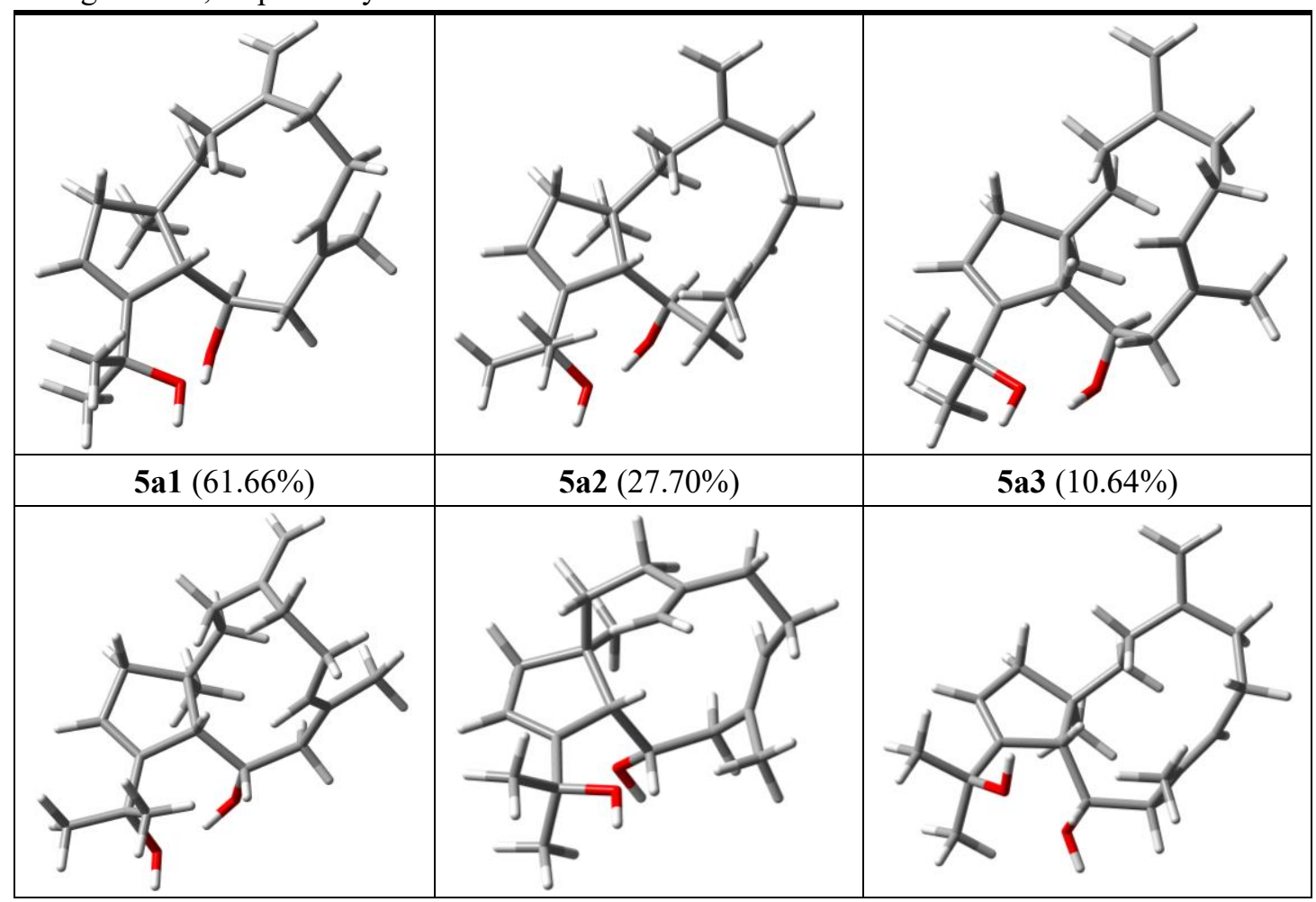


$\mathbf{5 b 1}(49.94 \%)$

$\mathbf{5 b 2}(40.40 \%)$

$\mathbf{5 b 3}(9.66 \%)$

Table S14. Important thermodynamic parameters (a.u.) of the optimized compound $\mathbf{5}$ with simplified structures at B3LYP/6-31+G(d,p) level in the gas phase.

\begin{tabular}{|c|c|c|}
\hline NO. & E + ZPE & G \\
\hline $\mathbf{5 a 1}$ & -931.344939 & -931.395493 \\
\hline $\mathbf{5 a 2}$ & -931.344904 & -931.394738 \\
\hline $\mathbf{5 a 3}$ & -931.344248 & -931.393835 \\
\hline $\mathbf{5 b 1}$ & -931.338141 & -931.388014 \\
\hline $\mathbf{5 b 2}$ & -931.337956 & -931.387814 \\
\hline $\mathbf{5 b 3}$ & -931.337217 & -931.386464 \\
\hline
\end{tabular}

Table S15. Optimized Z-Matrixes of compound 5 with simplified structures in the Gas Phase $(\AA)$ at B3LYP/6-31+G(d,p) level.

\begin{tabular}{|c|c|c|c|c|c|c|c|c|c|c|c|}
\hline \multicolumn{4}{|c|}{$5 a 1$} & \multicolumn{4}{|c|}{$5 \mathbf{a} 2$} & \multicolumn{4}{|c|}{$5 \mathbf{a} 3$} \\
\hline $\mathrm{C}$ & -2.15145 & -2.01162 & -0.32517 & $\mathrm{C}$ & -2.02001 & -2.00543 & -0.67231 & $\mathrm{C}$ & -2.10946 & -2.06919 & -0.08214 \\
\hline $\mathrm{C}$ & -2.13575 & -0.67237 & -0.29563 & $\mathrm{C}$ & -2.0717 & -0.71218 & -0.32059 & $\mathrm{C}$ & -2.12225 & -0.73656 & -0.21648 \\
\hline $\mathrm{C}$ & -0.76689 & -0.17457 & 0.203556 & $\mathrm{C}$ & -0.70544 & -0.28116 & 0.252513 & $\mathrm{C}$ & -0.75907 & -0.15342 & 0.200844 \\
\hline $\mathrm{C}$ & -0.16485 & -1.46 & 0.899848 & $\mathrm{C}$ & -0.03527 & -1.66124 & 0.621485 & $\mathrm{C}$ & -0.12043 & -1.33355 & 1.037315 \\
\hline $\mathrm{C}$ & -0.86541 & -2.62823 & 0.148118 & $\mathrm{C}$ & -0.68722 & -2.64123 & -0.39072 & $\mathrm{C}$ & -0.80544 & -2.59787 & 0.443437 \\
\hline $\mathrm{C}$ & -0.6626 & 1.130953 & 1.058611 & $\mathrm{C}$ & -0.58641 & 0.778207 & 1.39047 & $\mathrm{C}$ & -0.68423 & 1.248179 & 0.891289 \\
\hline $\mathrm{C}$ & 0.042699 & 2.287608 & 0.292716 & $\mathrm{C}$ & -0.25182 & 2.20468 & 0.876358 & $\mathrm{C}$ & -0.06686 & 2.337524 & -0.03718 \\
\hline $\mathrm{C}$ & 2.016517 & -1.61382 & -0.56496 & $\mathrm{C}$ & 2.170727 & -1.17788 & -0.7606 & $\mathrm{C}$ & 2.05562 & -1.61086 & -0.42687 \\
\hline $\mathrm{C}$ & 1.381152 & -1.49153 & 0.837008 & $\mathrm{C}$ & 1.513165 & -1.61793 & 0.564606 & $\mathrm{C}$ & 1.426386 & -1.34799 & 0.958528 \\
\hline $\mathrm{C}$ & 1.544842 & 2.103269 & 0.190049 & $\mathrm{C}$ & 1.048219 & 2.254732 & 0.077056 & $\mathrm{C}$ & 1.442814 & 2.232265 & -0.11565 \\
\hline $\mathrm{C}$ & 2.112336 & 1.652718 & -0.94201 & $\mathrm{C}$ & 2.196016 & 1.827913 & 0.628943 & $\mathrm{C}$ & 2.046365 & 1.558496 & -1.10934 \\
\hline $\mathrm{C}$ & 3.561088 & 1.339308 & -1.20502 & $\mathrm{C}$ & 3.554777 & 1.639429 & 0.00694 & $\mathrm{C}$ & 3.517945 & 1.224305 & -1.22045 \\
\hline $\mathrm{C}$ & 3.838693 & -0.1217 & -1.65727 & $\mathrm{C}$ & 4.151335 & 0.221736 & 0.248955 & $\mathrm{C}$ & 4.026107 & 0.11255 & -0.25486 \\
\hline $\mathrm{C}$ & 3.478829 & -1.18774 & -0.63869 & $\mathrm{C}$ & 3.659184 & -0.89398 & -0.66024 & $\mathrm{C}$ & 3.539367 & -1.30053 & -0.52122 \\
\hline $\mathrm{C}$ & -0.59535 & -1.54404 & 2.381379 & $\mathrm{C}$ & -0.44722 & -2.11973 & 2.038061 & $\mathrm{C}$ & -0.53052 & -1.24648 & 2.524314 \\
\hline $\mathrm{H}$ & -0.17463 & 0.009868 & -0.70208 & $\mathrm{H}$ & -0.1462 & 0.127219 & -0.59907 & $\mathrm{H}$ & -0.17804 & -0.06671 & -0.72629 \\
\hline $\mathrm{O}$ & -1.89832 & 1.558489 & 1.616892 & $\mathrm{O}$ & -1.66614 & 0.773424 & 2.30502 & $\mathrm{O}$ & -1.91684 & 1.677171 & 1.454446 \\
\hline $\mathrm{C}$ & -3.29932 & 0.163992 & -0.82617 & $\mathrm{C}$ & -3.22761 & 0.197246 & -0.72718 & $\mathrm{C}$ & -3.31097 & 0.001221 & -0.83131 \\
\hline $\mathrm{C}$ & -4.49946 & 0.092316 & 0.134936 & $\mathrm{C}$ & -4.58196 & -0.52552 & -0.66448 & $\mathrm{C}$ & -4.51861 & -0.03143 & 0.122175 \\
\hline $\mathrm{C}$ & -3.7017 & -0.2763 & -2.24341 & $\mathrm{C}$ & -2.98241 & 0.76845 & -2.13755 & $\mathrm{C}$ & -3.67683 & -0.58327 & -2.20614 \\
\hline $\mathrm{O}$ & -2.84597 & 1.540209 & -0.91435 & $\mathrm{O}$ & -3.27274 & 1.305878 & 0.202792 & $\mathrm{O}$ & -2.91454 & 1.3808 & -1.04082 \\
\hline $\mathrm{C}$ & 4.416691 & -1.73513 & 0.142199 & $\mathrm{C}$ & 4.537028 & -1.61005 & -1.37303 & $\mathrm{C}$ & 4.414124 & -2.2624 & -0.83981 \\
\hline $\mathrm{C}$ & 2.309978 & 2.453235 & 1.445635 & $\mathrm{C}$ & 0.928051 & 2.754716 & -1.34173 & $\mathrm{C}$ & 2.189024 & 2.879524 & 1.027584 \\
\hline $\mathrm{H}$ & -2.97107 & -2.6125 & -0.70998 & $\mathrm{H}$ & -2.81323 & -2.54156 & -1.18434 & $\mathrm{H}$ & -2.92107 & -2.72868 & -0.3774 \\
\hline $\mathrm{H}$ & -0.27881 & -2.97344 & -0.71543 & $\mathrm{H}$ & -0.76269 & -3.66183 & 0.004717 & $\mathrm{H}$ & -0.22334 & -3.03475 & -0.38054 \\
\hline $\mathrm{H}$ & -1.01173 & -3.50672 & 0.789135 & $\mathrm{H}$ & -0.10815 & -2.71292 & -1.32384 & $\mathrm{H}$ & -0.92529 & -3.39423 & 1.188766 \\
\hline $\mathrm{H}$ & -0.04173 & 0.907467 & 1.929448 & $\mathrm{H}$ & 0.276581 & 0.476956 & 1.991829 & $\mathrm{H}$ & -0.01402 & 1.156958 & 1.750334 \\
\hline $\mathrm{H}$ & -0.17836 & 3.205476 & 0.852106 & $\mathrm{H}$ & -0.19288 & 2.851949 & 1.759662 & $\mathrm{H}$ & -0.52216 & 2.267322 & -1.02975 \\
\hline $\mathrm{H}$ & -0.40873 & 2.389587 & -0.69946 & $\mathrm{H}$ & -1.0843 & 2.575693 & 0.27022 & $\mathrm{H}$ & -0.35132 & 3.306577 & 0.390632 \\
\hline
\end{tabular}




\begin{tabular}{|c|c|c|c|c|c|c|c|c|c|c|c|}
\hline $\mathrm{H}$ & 1.935858 & -2.65193 & -0.91355 & $\mathrm{H}$ & 2.014453 & -1.94493 & -1.52727 & $\mathrm{H}$ & 1.527876 & -1.01838 & -1.18255 \\
\hline $\mathrm{H}$ & 1.446245 & -1.01829 & -1.28245 & $\mathrm{H}$ & 1.677778 & -0.271 & -1.12626 & $\mathrm{H}$ & 1.906384 & -2.65953 & -0.70549 \\
\hline $\mathrm{H}$ & 1.740798 & -2.31718 & 1.464586 & $\mathrm{H}$ & 1.89645 & -2.6125 & 0.829253 & $\mathrm{H}$ & 1.808344 & -2.10135 & 1.660259 \\
\hline $\mathrm{H}$ & 1.759386 & -0.578 & 1.30944 & $\mathrm{H}$ & 1.853505 & -0.9524 & 1.365803 & $\mathrm{H}$ & 1.781007 & -0.38326 & 1.338875 \\
\hline $\mathrm{H}$ & 1.451825 & 1.482914 & -1.79471 & $\mathrm{H}$ & 2.160908 & 1.534905 & 1.679962 & $\mathrm{H}$ & 1.410638 & 1.172769 & -1.90547 \\
\hline $\mathrm{H}$ & 3.929874 & 1.999085 & -2.00461 & $\mathrm{H}$ & 3.54794 & 1.852788 & -1.0665 & $\mathrm{H}$ & 4.123082 & 2.118353 & -1.02381 \\
\hline $\mathrm{H}$ & 4.176446 & 1.548242 & -0.32551 & $\mathrm{H}$ & 4.254669 & 2.357905 & 0.458199 & $\mathrm{H}$ & 3.747692 & 0.92502 & -2.24923 \\
\hline $\mathrm{H}$ & 4.904766 & -0.19619 & -1.90027 & $\mathrm{H}$ & 3.96772 & -0.04724 & 1.299233 & $\mathrm{H}$ & 5.121284 & 0.107323 & -0.29604 \\
\hline $\mathrm{H}$ & 3.291099 & -0.31235 & -2.59107 & $\mathrm{H}$ & 5.239708 & 0.282823 & 0.143117 & $\mathrm{H}$ & 3.760965 & 0.406787 & 0.768653 \\
\hline $\mathrm{H}$ & -0.11174 & -0.78561 & 3.003125 & $\mathrm{H}$ & -0.03053 & -3.11353 & 2.23803 & $\mathrm{H}$ & -1.61348 & -1.13893 & 2.629513 \\
\hline $\mathrm{H}$ & -0.31858 & -2.52129 & 2.791847 & $\mathrm{H}$ & -1.53406 & -2.17495 & 2.134774 & $\mathrm{H}$ & -0.22123 & -2.1577 & 3.047928 \\
\hline $\mathrm{H}$ & -1.67669 & -1.4204 & 2.486623 & $\mathrm{H}$ & -0.08624 & -1.44406 & 2.817007 & $\mathrm{H}$ & -0.06249 & -0.40361 & 3.040182 \\
\hline $\mathrm{H}$ & -2.42437 & 1.853894 & 0.853475 & $\mathrm{H}$ & -2.45468 & 1.013512 & 1.789276 & $\mathrm{H}$ & -2.48377 & 1.86779 & 0.687024 \\
\hline $\mathrm{H}$ & -4.87838 & -0.93117 & 0.202406 & $\mathrm{H}$ & -4.75721 & -0.91921 & 0.339367 & $\mathrm{H}$ & -4.85534 & -1.05899 & 0.284378 \\
\hline $\mathrm{H}$ & -5.3173 & 0.733617 & -0.21819 & $\mathrm{H}$ & -4.639 & -1.35064 & -1.37839 & $\mathrm{H}$ & -5.35849 & 0.537867 & -0.29639 \\
\hline $\mathrm{H}$ & -4.19737 & 0.41425 & 1.133694 & $\mathrm{H}$ & -5.38952 & 0.173903 & -0.91311 & $\mathrm{H}$ & -4.24325 & 0.396378 & 1.088624 \\
\hline $\mathrm{H}$ & -4.5178 & 0.353432 & -2.62062 & $\mathrm{H}$ & -2.95806 & -0.03203 & -2.88175 & $\mathrm{H}$ & -3.98438 & -1.62875 & -2.12826 \\
\hline $\mathrm{H}$ & -2.85113 & -0.18832 & -2.92402 & $\mathrm{H}$ & -2.03266 & 1.307621 & -2.17678 & $\mathrm{H}$ & -4.51155 & -0.02447 & -2.64889 \\
\hline $\mathrm{H}$ & -4.05162 & -1.31114 & -2.25756 & $\mathrm{H}$ & -3.78224 & 1.467139 & -2.41388 & $\mathrm{H}$ & -2.82095 & -0.52347 & -2.88324 \\
\hline $\mathrm{H}$ & -3.56572 & 2.05618 & -1.30299 & $\mathrm{H}$ & -4.02568 & 1.864914 & -0.03052 & $\mathrm{H}$ & -3.65187 & 1.83029 & -1.47611 \\
\hline $\mathrm{H}$ & 5.459786 & -1.43789 & 0.071947 & $\mathrm{H}$ & 4.218284 & -2.42232 & -2.02101 & $\mathrm{H}$ & 4.096262 & -3.28495 & -1.02596 \\
\hline $\mathrm{H}$ & 4.181581 & -2.4991 & 0.878342 & $\mathrm{H}$ & 5.605074 & -1.41282 & -1.33325 & $\mathrm{H}$ & 5.478987 & -2.06286 & -0.92588 \\
\hline $\mathrm{H}$ & 1.860788 & 1.986341 & 2.330024 & $\mathrm{H}$ & 1.878324 & 2.766339 & -1.87903 & $\mathrm{H}$ & 1.875809 & 2.470827 & 1.996731 \\
\hline $\mathrm{H}$ & 2.271915 & 3.53688 & 1.619252 & $\mathrm{H}$ & 0.220945 & 2.14443 & -1.9198 & $\mathrm{H}$ & 1.964045 & 3.953336 & 1.063175 \\
\hline $\mathrm{H}$ & 3.359335 & 2.156598 & 1.412265 & $\mathrm{H}$ & 0.527427 & 3.776722 & -1.34979 & $\mathrm{H}$ & 3.272073 & 2.766104 & 0.952844 \\
\hline \multicolumn{4}{|c|}{$5 \mathrm{~b} 1$} & \multicolumn{4}{|c|}{$5 \mathrm{~b} 2$} & \multicolumn{4}{|c|}{$5 b 3$} \\
\hline $\mathrm{C}$ & 2.300235 & -1.88364 & -0.29829 & $\mathrm{C}$ & 2.518144 & 1.55261 & -0.25313 & $\mathrm{C}$ & -2.03207 & -2.09874 & 0.083926 \\
\hline $\mathrm{C}$ & 2.206972 & -0.61259 & 0.110833 & $\mathrm{C}$ & 1.971656 & 0.336101 & -0.33811 & $\mathrm{C}$ & -2.10169 & -0.78842 & -0.17666 \\
\hline $\mathrm{C}$ & 0.805905 & -0.04824 & -0.20961 & $\mathrm{C}$ & 0.61002 & 0.280331 & 0.361421 & $\mathrm{C}$ & -0.81436 & -0.07498 & 0.279744 \\
\hline $\mathrm{C}$ & 0.223495 & -1.09084 & -1.24643 & $\mathrm{C}$ & 0.259311 & 1.815442 & 0.562397 & $\mathrm{C}$ & -0.06548 & -1.16316 & 1.161493 \\
\hline $\mathrm{C}$ & 1.045074 & -2.3847 & -0.9483 & $\mathrm{C}$ & 1.654514 & 2.516277 & 0.50464 & $\mathrm{C}$ & -0.75246 & -2.50547 & 0.747231 \\
\hline $\mathrm{C}$ & 0.753019 & 1.478591 & -0.52071 & $\mathrm{C}$ & 0.654965 & -0.66683 & 1.587153 & $\mathrm{C}$ & -1.01416 & 1.378019 & 0.778672 \\
\hline $\mathrm{C}$ & -0.58966 & 2.090912 & -0.967 & $\mathrm{C}$ & -0.71698 & -1.06434 & 2.199748 & $\mathrm{C}$ & 0.264336 & 2.220153 & 1.04066 \\
\hline $\mathrm{C}$ & -1.7789 & -1.85169 & 0.291189 & $\mathrm{C}$ & -2.04228 & 1.903118 & -0.79355 & $\mathrm{C}$ & 1.97286 & -1.29359 & -0.52422 \\
\hline $\mathrm{C}$ & -1.30378 & -1.31531 & -1.08598 & $\mathrm{C}$ & -0.56395 & 2.332382 & -0.66106 & $\mathrm{C}$ & 1.472866 & -1.24591 & 0.935218 \\
\hline $\mathrm{C}$ & -1.87936 & 1.876522 & -0.1989 & $\mathrm{C}$ & -1.69081 & -1.60711 & 1.174978 & $\mathrm{C}$ & 1.320727 & 2.164194 & -0.04464 \\
\hline $\mathrm{C}$ & -1.95282 & 1.384934 & 1.048086 & $\mathrm{C}$ & -2.80872 & -0.92214 & 0.884013 & $\mathrm{C}$ & 2.553563 & 1.745196 & 0.280148 \\
\hline $\mathrm{C}$ & -3.19129 & 0.963717 & 1.791658 & $\mathrm{C}$ & -3.74633 & -1.14299 & -0.2699 & $\mathrm{C}$ & 3.719955 & 1.364083 & -0.5889 \\
\hline $\mathrm{C}$ & -3.28216 & -0.57706 & 1.999256 & $\mathrm{C}$ & -3.80114 & 0.089737 & -1.22203 & $\mathrm{C}$ & 4.26705 & -0.04262 & -0.21078 \\
\hline $\mathrm{C}$ & -3.17296 & -1.40717 & 0.727579 & $\mathrm{C}$ & -2.43275 & 0.65813 & -1.57967 & $\mathrm{C}$ & 3.480308 & -1.24903 & -0.70389 \\
\hline $\mathrm{C}$ & 0.521247 & -0.69792 & -2.7094 & $\mathrm{C}$ & -0.43775 & 2.212431 & 1.874234 & $\mathrm{C}$ & -0.30785 & -0.97144 & 2.672557 \\
\hline $\mathrm{H}$ & 0.227383 & -0.16247 & 0.715108 & $\mathrm{H}$ & -0.12176 & -0.1723 & -0.31179 & $\mathrm{H}$ & -0.22544 & 0.067585 & -0.63445 \\
\hline $\mathrm{O}$ & 1.663664 & 1.862613 & -1.54359 & $\mathrm{O}$ & 1.513716 & -0.09855 & 2.585629 & $\mathrm{O}$ & -1.82929 & 1.384496 & 1.959902 \\
\hline
\end{tabular}




\begin{tabular}{|c|c|c|c|c|c|c|c|c|c|c|c|}
\hline $\mathrm{C}$ & 3.258324 & 0.084061 & 0.965048 & $\mathrm{C}$ & 2.641581 & -0.86485 & -0.99432 & $\mathrm{C}$ & -3.21425 & -0.11282 & -0.97956 \\
\hline $\mathrm{C}$ & 4.543012 & -0.7418 & 1.124127 & $\mathrm{C}$ & 3.181158 & -0.512 & -2.39084 & $\mathrm{C}$ & -4.2116 & 0.640875 & -0.0875 \\
\hline $\mathrm{C}$ & 2.684578 & 0.428831 & 2.353031 & $\mathrm{C}$ & 3.774576 & -1.40482 & -0.09922 & $\mathrm{C}$ & -3.98312 & -1.11195 & -1.85993 \\
\hline $\mathrm{O}$ & 3.615668 & 1.313532 & 0.27963 & $\mathrm{O}$ & 1.626697 & -1.87899 & -1.14682 & $\mathrm{O}$ & -2.6357 & 0.891092 & -1.85235 \\
\hline $\mathrm{C}$ & -4.26587 & -1.75389 & 0.038882 & $\mathrm{C}$ & -1.70061 & 0.119124 & -2.55895 & $\mathrm{C}$ & 4.120426 & -2.255 & -1.31181 \\
\hline $\mathrm{C}$ & -3.09041 & 2.261457 & -1.02175 & $\mathrm{C}$ & -1.26994 & -2.88631 & 0.489408 & $\mathrm{C}$ & 0.895401 & 2.579582 & -1.43269 \\
\hline $\mathrm{H}$ & 3.154739 & -2.53048 & -0.12894 & $\mathrm{H}$ & 3.490173 & 1.830382 & -0.65196 & $\mathrm{H}$ & -2.78109 & -2.82885 & -0.2059 \\
\hline $\mathrm{H}$ & 0.518345 & -3.05967 & -0.25835 & $\mathrm{H}$ & 2.056144 & 2.679831 & 1.514398 & $\mathrm{H}$ & -0.13412 & -3.08171 & 0.04305 \\
\hline $\mathrm{H}$ & 1.232489 & -2.96829 & -1.8585 & $\mathrm{H}$ & 1.5933 & 3.504553 & 0.029555 & $\mathrm{H}$ & -0.90319 & -3.16093 & 1.615124 \\
\hline $\mathrm{H}$ & 1.038548 & 1.97785 & 0.419174 & $\mathrm{H}$ & 1.107296 & -1.5943 & 1.214304 & $\mathrm{H}$ & -1.56279 & 1.868654 & -0.03343 \\
\hline $\mathrm{H}$ & -0.40192 & 3.170398 & -1.05997 & $\mathrm{H}$ & -0.50528 & -1.8315 & 2.963279 & $\mathrm{H}$ & 0.701348 & 1.907624 & 1.993636 \\
\hline $\mathrm{H}$ & -0.7695 & 1.768289 & -1.99892 & $\mathrm{H}$ & -1.15025 & -0.20976 & 2.722992 & $\mathrm{H}$ & -0.0818 & 3.258294 & 1.179543 \\
\hline $\mathrm{H}$ & -1.06783 & -1.56062 & 1.069199 & $\mathrm{H}$ & -2.58969 & 2.732669 & -1.26852 & $\mathrm{H}$ & 1.552496 & -0.44594 & -1.07602 \\
\hline $\mathrm{H}$ & -1.75427 & -2.94869 & 0.275795 & $\mathrm{H}$ & -2.48796 & 1.832836 & 0.203221 & $\mathrm{H}$ & 1.594294 & -2.1962 & -1.01692 \\
\hline $\mathrm{H}$ & -1.81799 & -0.37567 & -1.29263 & $\mathrm{H}$ & -0.56044 & 3.427586 & -0.59002 & $\mathrm{H}$ & 1.829353 & -2.14861 & 1.450276 \\
\hline $\mathrm{H}$ & -1.62899 & -2.01229 & -1.86916 & $\mathrm{H}$ & -0.01362 & 2.097622 & -1.57941 & $\mathrm{H}$ & 1.944728 & -0.40764 & 1.451334 \\
\hline $\mathrm{H}$ & -1.02285 & 1.178972 & 1.575834 & $\mathrm{H}$ & -3.02314 & -0.03732 & 1.484686 & $\mathrm{H}$ & 2.739154 & 1.562939 & 1.339498 \\
\hline $\mathrm{H}$ & -3.21786 & 1.433602 & 2.785175 & $\mathrm{H}$ & -4.76847 & -1.34432 & 0.08199 & $\mathrm{H}$ & 4.542012 & 2.079397 & -0.44227 \\
\hline $\mathrm{H}$ & -4.09356 & 1.289 & 1.267622 & $\mathrm{H}$ & -3.4367 & -2.0142 & -0.85328 & $\mathrm{H}$ & 3.469155 & 1.395087 & -1.65439 \\
\hline $\mathrm{H}$ & -4.23689 & -0.78898 & 2.494786 & $\mathrm{H}$ & -4.40355 & 0.87617 & -0.74985 & $\mathrm{H}$ & 4.34376 & -0.08282 & 0.885982 \\
\hline $\mathrm{H}$ & -2.49397 & -0.88603 & 2.698541 & $\mathrm{H}$ & -4.33686 & -0.20663 & -2.13112 & $\mathrm{H}$ & 5.292589 & -0.13411 & -0.58424 \\
\hline $\mathrm{H}$ & 0.208748 & -1.51165 & -3.37466 & $\mathrm{H}$ & 0.145983 & 1.880179 & 2.734322 & $\mathrm{H}$ & 0.097788 & -0.02802 & 3.044 \\
\hline $\mathrm{H}$ & 1.587861 & -0.51695 & -2.85864 & $\mathrm{H}$ & -0.52789 & 3.304991 & 1.920365 & $\mathrm{H}$ & 0.17442 & -1.78809 & 3.223215 \\
\hline $\mathrm{H}$ & -0.00316 & 0.206312 & -3.02121 & $\mathrm{H}$ & -1.44638 & 1.804004 & 1.970693 & $\mathrm{H}$ & -1.37494 & -0.97902 & 2.904941 \\
\hline $\mathrm{H}$ & 2.547233 & 1.774716 & -1.14821 & $\mathrm{H}$ & 1.565545 & -0.72816 & 3.317035 & $\mathrm{H}$ & -2.00566 & 2.308623 & 2.182468 \\
\hline $\mathrm{H}$ & 4.970759 & -0.97722 & 0.146976 & $\mathrm{H}$ & 2.366286 & -0.16882 & -3.03292 & $\mathrm{H}$ & -4.90299 & 1.210382 & -0.71629 \\
\hline $\mathrm{H}$ & 5.281846 & -0.16263 & 1.689444 & $\mathrm{H}$ & 3.941662 & 0.272229 & -2.34577 & $\mathrm{H}$ & -3.70549 & 1.313483 & 0.603377 \\
\hline $\mathrm{H}$ & 4.365433 & -1.67279 & 1.66921 & $\mathrm{H}$ & 3.641955 & -1.39382 & -2.85403 & $\mathrm{H}$ & -4.7837 & -0.0744 & 0.510168 \\
\hline $\mathrm{H}$ & 3.437546 & 0.948314 & 2.958985 & $\mathrm{H}$ & 4.203018 & -2.32044 & -0.52779 & $\mathrm{H}$ & -3.30393 & -1.65814 & -2.52373 \\
\hline $\mathrm{H}$ & 1.80804 & 1.075494 & 2.273636 & $\mathrm{H}$ & 3.404644 & -1.6244 & 0.904678 & $\mathrm{H}$ & -4.7061 & -0.5657 & -2.47223 \\
\hline $\mathrm{H}$ & 2.396762 & -0.48061 & 2.888209 & $\mathrm{H}$ & 4.576922 & -0.66746 & -0.00599 & $\mathrm{H}$ & -4.52859 & -1.84626 & -1.26088 \\
\hline $\mathrm{H}$ & 4.153647 & 1.851145 & 0.876101 & $\mathrm{H}$ & 2.052101 & -2.64866 & -1.54786 & $\mathrm{H}$ & -2.08807 & 0.422148 & -2.49715 \\
\hline $\mathrm{H}$ & -5.26068 & -1.45298 & 0.357371 & $\mathrm{H}$ & -2.06302 & -0.7332 & -3.12778 & $\mathrm{H}$ & 5.193452 & -2.23225 & -1.48321 \\
\hline $\mathrm{H}$ & -4.20904 & -2.34916 & -0.86877 & $\mathrm{H}$ & -0.71619 & 0.489078 & -2.82598 & $\mathrm{H}$ & 3.593804 & -3.14062 & -1.65733 \\
\hline $\mathrm{H}$ & -2.94431 & 3.256733 & -1.45989 & $\mathrm{H}$ & -2.06832 & -3.32533 & -0.11144 & $\mathrm{H}$ & 0.636294 & 3.64706 & -1.43898 \\
\hline $\mathrm{H}$ & -4.01834 & 2.282508 & -0.44942 & $\mathrm{H}$ & -0.97487 & -3.63178 & 1.239773 & $\mathrm{H}$ & -0.00246 & 2.04929 & -1.76977 \\
\hline $\mathrm{H}$ & -3.23077 & 1.569491 & -1.86226 & $\mathrm{H}$ & -0.40192 & -2.72522 & -0.15952 & $\mathrm{H}$ & 1.682923 & 2.430175 & -2.17435 \\
\hline
\end{tabular}



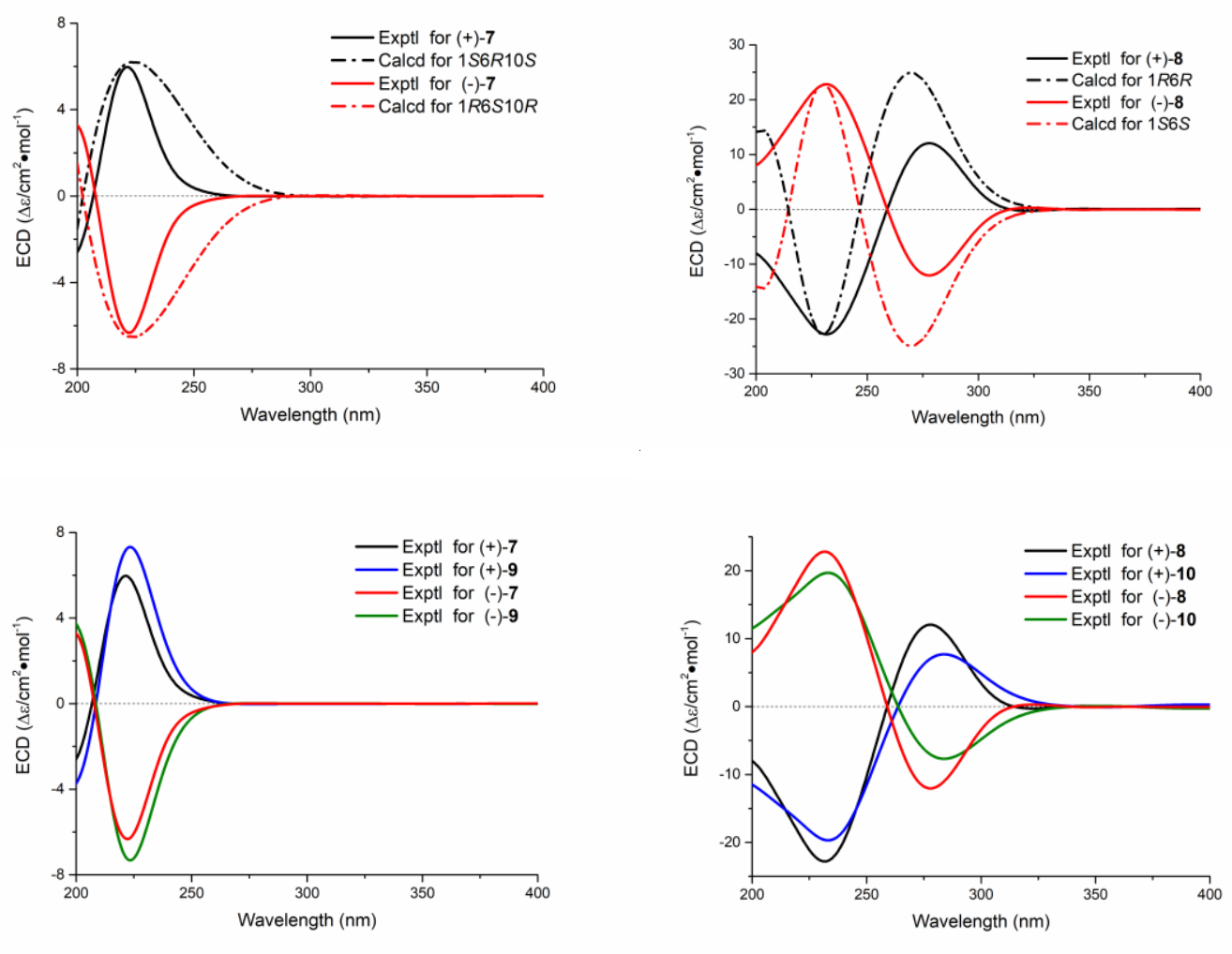

Figure S8 Experimental and calculated ECD spectra of 7-10

Table S16. Stable conformers of compound 7 with $(1 S, 6 R, 9 S)-7 \mathbf{a}$ and $(1 R, 6 S, 9 R)-7 \mathbf{b}$ configurations, respectively.

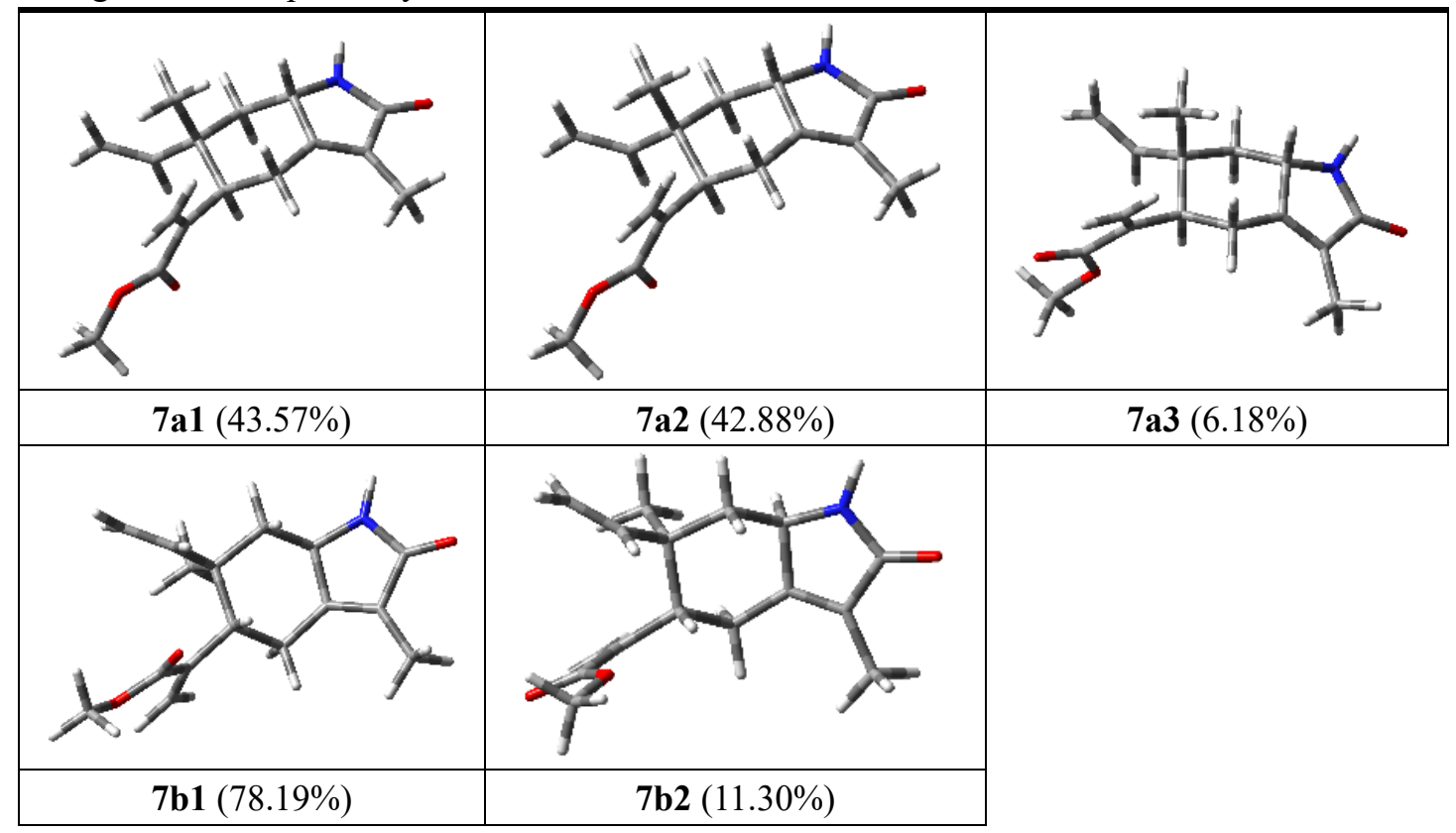

Table S17. Important thermodynamic parameters (a.u.) of the optimized compound 7 with structures at B3LYP/6-31+G(d,p) level in the gas phase.

\begin{tabular}{l|c|c|c|c|c}
\hline conformations & E+ZPE & G & conformations & E+ZPE & G \\
\hline
\end{tabular}




\begin{tabular}{l|l|l|l|l|l}
\hline $\mathbf{7 a 1}$ & -902.436537 & -902.485758 & $\mathbf{7 b 1}$ & -902.436533 & -902.485746 \\
\hline $\mathbf{7 a 2}$ & -902.436532 & -902.485743 & $\mathbf{7 b 2}$ & -902.434917 & -902.483921 \\
\hline $\mathbf{7 a 3}$ & -902.434916 & -902.483916 & & & \\
\hline
\end{tabular}

Table S18. Optimized Z-Matrixes of compound 7 with structures in the Gas Phase ( $\AA$ ) at B3LYP/6$31+\mathrm{G}(\mathrm{d}, \mathrm{p})$ level.

\begin{tabular}{|c|c|c|c|c|c|c|c|c|c|c|c|}
\hline \multicolumn{4}{|c|}{$7 \mathrm{a} 1$} & \multicolumn{4}{|c|}{$7 \mathrm{a} 2$} & \multicolumn{4}{|c|}{$7 \mathrm{a} 3$} \\
\hline $\mathrm{C}$ & 2.7283 & 2.0671 & -0.8795 & $\mathrm{C}$ & 2.5652 & 2.3675 & -0.3241 & $\mathrm{C}$ & 2.7734 & 2.1323 & -0.7263 \\
\hline $\mathrm{C}$ & 1.6933 & -2.5006 & -0.2018 & $\mathrm{C}$ & 1.8489 & -2.3397 & -0.4936 & $\mathrm{C}$ & 1.8185 & -2.4723 & -0.6575 \\
\hline $\mathrm{C}$ & 1.4361 & -1.3568 & 0.4625 & $\mathrm{C}$ & 1.5223 & -1.2979 & 0.2941 & $\mathrm{C}$ & 1.5895 & -1.3921 & 0.1135 \\
\hline $\mathrm{C}$ & 0.2327 & -0.4469 & 0.1967 & $\mathrm{C}$ & 0.2471 & -0.4589 & 0.1887 & $\mathrm{C}$ & 0.3494 & -0.4988 & 0.052 \\
\hline $\mathrm{C}$ & 0.5162 & 0.7298 & -0.8305 & $\mathrm{C}$ & 0.4482 & 0.9043 & -0.6014 & $\mathrm{C}$ & 0.5513 & 0.8096 & -0.825 \\
\hline $\mathrm{C}$ & 1.6052 & 1.6543 & -0.2756 & $\mathrm{C}$ & 1.4478 & 1.7956 & 0.1455 & $\mathrm{C}$ & 1.626 & 1.698 & -0.1869 \\
\hline $\mathrm{C}$ & -1.0462 & -1.2527 & -0.1659 & $\mathrm{C}$ & -0.9675 & -1.2739 & -0.3393 & $\mathrm{C}$ & -0.9297 & -1.2864 & -0.3502 \\
\hline $\mathrm{C}$ & -2.2001 & -0.328 & -0.3677 & $\mathrm{C}$ & -2.1843 & -0.4117 & -0.4161 & $\mathrm{C}$ & -2.1124 & -0.3764 & -0.3886 \\
\hline $\mathrm{C}$ & -2.0058 & 0.7973 & -1.333 & $\mathrm{C}$ & -2.0599 & 0.8751 & -1.1667 & $\mathrm{C}$ & -1.991 & 0.8553 & -1.227 \\
\hline $\mathrm{C}$ & -0.7685 & 1.6102 & -0.966 & $\mathrm{C}$ & -0.8986 & 1.696 & -0.6167 & $\mathrm{C}$ & -0.7625 & 1.6562 & -0.808 \\
\hline $\mathrm{C}$ & -3.3923 & -0.2971 & 0.2452 & $\mathrm{C}$ & -3.389 & -0.5725 & 0.1523 & $\mathrm{C}$ & -3.2765 & -0.4485 & 0.2744 \\
\hline $\mathrm{C}$ & -4.0857 & 0.8965 & -0.2796 & $\mathrm{C}$ & -4.1632 & 0.6396 & -0.1844 & $\mathrm{C}$ & -4.0229 & 0.7753 & -0.0823 \\
\hline $\mathrm{N}$ & -3.2304 & 1.5092 & -1.1614 & $\mathrm{~N}$ & -3.3396 & 1.456 & -0.9177 & $\mathrm{~N}$ & -3.226 & 1.5053 & -0.9276 \\
\hline $\mathrm{C}$ & 0.8917 & 0.1736 & -2.2266 & $\mathrm{C}$ & 0.8918 & 0.6376 & -2.0609 & $\mathrm{C}$ & 0.8921 & 0.4517 & -2.2927 \\
\hline $\mathrm{C}$ & -4.0074 & -1.1681 & 1.2714 & $\mathrm{C}$ & -3.9585 & -1.6457 & 0.9957 & $\mathrm{C}$ & -3.8271 & -1.4398 & 1.2242 \\
\hline $\mathrm{C}$ & 2.3118 & -0.9538 & 1.6123 & $\mathrm{C}$ & 2.4291 & -0.9497 & 1.4319 & $\mathrm{C}$ & 2.6645 & -1.0919 & 1.1086 \\
\hline $\mathrm{O}$ & 3.5115 & -1.5891 & 1.5699 & $\mathrm{O}$ & 3.7272 & -1.161 & 1.0935 & $\mathrm{O}$ & 2.1398 & -0.482 & 2.2008 \\
\hline $\mathrm{O}$ & 1.9716 & -0.1468 & 2.4652 & $\mathrm{O}$ & 2.0327 & -0.4942 & 2.4943 & $\mathrm{O}$ & 3.8404 & -1.3908 & 0.9586 \\
\hline $\mathrm{C}$ & 4.3897 & -1.2569 & 2.647 & $\mathrm{C}$ & 4.6584 & -0.7653 & 2.0999 & $\mathrm{C}$ & 3.1021 & -0.1751 & 3.2098 \\
\hline $\mathrm{O}$ & -5.2044 & 1.2659 & 0.0197 & $\mathrm{O}$ & -5.3132 & 0.8685 & 0.1354 & $\mathrm{O}$ & -5.1335 & 1.0771 & 0.3077 \\
\hline $\mathrm{H}$ & -1.966 & 0.4357 & -2.3652 & $\mathrm{H}$ & -1.969 & 0.7042 & -2.2441 & $\mathrm{H}$ & -1.9821 & 0.6156 & -2.2951 \\
\hline $\mathrm{H}$ & -0.0208 & 0.0345 & 1.1542 & $\mathrm{H}$ & -0.0391 & -0.1852 & 1.2168 & $\mathrm{H}$ & 0.1366 & -0.1578 & 1.0763 \\
\hline $\mathrm{H}$ & 3.4133 & 2.7328 & -0.361 & $\mathrm{H}$ & 3.1831 & 2.9767 & 0.3304 & $\mathrm{H}$ & 3.4481 & 2.7596 & -0.1496 \\
\hline $\mathrm{H}$ & 3.0037 & 1.7726 & -1.8852 & $\mathrm{H}$ & 2.904 & 2.2561 & -1.348 & $\mathrm{H}$ & 3.0868 & 1.8823 & -1.7341 \\
\hline $\mathrm{H}$ & 2.5359 & -3.1408 & 0.0458 & $\mathrm{H}$ & 2.7584 & -2.9147 & -0.3369 & $\mathrm{H}$ & 2.7279 & -3.0617 & -0.555 \\
\hline $\mathrm{H}$ & 1.0757 & -2.8445 & -1.0223 & $\mathrm{H}$ & 1.2324 & -2.6566 & -1.3267 & $\mathrm{H}$ & 1.1301 & -2.8012 & -1.4265 \\
\hline $\mathrm{H}$ & 1.43 & 2.0191 & 0.7399 & $\mathrm{H}$ & 1.2102 & 1.9783 & 1.1966 & $\mathrm{H}$ & 1.4208 & 2.009 & 0.8396 \\
\hline $\mathrm{H}$ & -0.9115 & -1.8298 & -1.0871 & $\mathrm{H}$ & -1.1387 & -2.1361 & 0.3157 & $\mathrm{H}$ & -0.8251 & -1.7429 & -1.3406 \\
\hline $\mathrm{H}$ & -1.259 & -1.9779 & 0.6272 & $\mathrm{H}$ & -0.7762 & -1.6748 & -1.3408 & $\mathrm{H}$ & -1.0898 & -2.1071 & 0.3581 \\
\hline $\mathrm{H}$ & -0.9525 & 2.1399 & -0.0191 & $\mathrm{H}$ & -1.1411 & 2.0261 & 0.4051 & $\mathrm{H}$ & -0.9254 & 2.0606 & 0.2027 \\
\hline $\mathrm{H}$ & -0.6187 & 2.3982 & -1.7176 & $\mathrm{H}$ & -0.7973 & 2.6183 & -1.205 & $\mathrm{H}$ & -0.6626 & 2.5343 & -1.4607 \\
\hline $\mathrm{H}$ & -3.5105 & 2.3405 & -1.6633 & $\mathrm{H}$ & -3.6728 & 2.3382 & -1.2819 & $\mathrm{H}$ & -3.5523 & 2.3753 & -1.3259 \\
\hline $\mathrm{H}$ & 0.9844 & 0.9807 & -2.9638 & $\mathrm{H}$ & 0.2119 & -0.0393 & -2.5861 & $\mathrm{H}$ & 0.9403 & 1.3523 & -2.9166 \\
\hline $\mathrm{H}$ & 0.1418 & -0.5215 & -2.6158 & $\mathrm{H}$ & 1.8897 & 0.1881 & -2.1095 & $\mathrm{H}$ & 0.1452 & -0.2073 & -2.7447 \\
\hline $\mathrm{H}$ & 1.8472 & -0.3623 & -2.2079 & $\mathrm{H}$ & 0.9235 & 1.5676 & -2.6413 & $\mathrm{H}$ & 1.8588 & -0.0561 & -2.3785 \\
\hline $\mathrm{H}$ & -4.2015 & -0.603 & 2.1888 & $\mathrm{H}$ & -4.2335 & -1.2541 & 1.9802 & $\mathrm{H}$ & -4.0518 & -0.9664 & 2.1853 \\
\hline
\end{tabular}




\begin{tabular}{|c|c|c|c|c|c|c|c|c|c|c|c|}
\hline $\mathrm{H}$ & -3.3655 & -2.0158 & 1.5297 & $\mathrm{H}$ & -4.8561 & -2.0624 & 0.5278 & $\mathrm{H}$ & -3.1285 & -2.2599 & 1.4122 \\
\hline $\mathrm{H}$ & -4.9576 & -1.5728 & 0.9079 & $\mathrm{H}$ & -3.2523 & -2.4668 & 1.1482 & $\mathrm{H}$ & -4.752 & -1.8731 & 0.8304 \\
\hline $\mathrm{H}$ & 4.7556 & -0.2324 & 2.5298 & $\mathrm{H}$ & 4.5539 & 0.3013 & 2.3232 & $\mathrm{H}$ & 3.5917 & -1.087 & 3.5661 \\
\hline $\mathrm{H}$ & 5.2444 & -1.9391 & 2.6124 & $\mathrm{H}$ & 4.5238 & -1.3669 & 3.004 & $\mathrm{H}$ & 2.5752 & 0.2849 & 4.0504 \\
\hline $\mathrm{H}$ & 3.8925 & -1.3862 & 3.6133 & $\mathrm{H}$ & 5.6672 & -0.9388 & 1.7151 & $\mathrm{H}$ & 3.8395 & 0.5389 & 2.8298 \\
\hline \multicolumn{4}{|c|}{$7 \mathrm{~b} 1$} & \multicolumn{4}{|c|}{$7 \mathrm{~b} 2$} & & & & \\
\hline $\mathrm{C}$ & 1.9937 & -2.3347 & 1.7293 & $\mathrm{C}$ & 2.0841 & -2.5174 & 1.5181 & & & & \\
\hline $\mathrm{C}$ & 2.058 & 0.1423 & -2.2351 & $\mathrm{C}$ & 2.0358 & -0.0426 & -2.3472 & & & & \\
\hline $\mathrm{C}$ & 1.7575 & 0.412 & -0.9501 & $\mathrm{C}$ & 1.7837 & 0.2043 & -1.0478 & & & & \\
\hline $\mathrm{C}$ & 0.3709 & 0.2521 & -0.3208 & $\mathrm{C}$ & 0.4153 & 0.1028 & -0.3746 & & & & \\
\hline $\mathrm{C}$ & 0.1345 & -1.1583 & 0.3666 & $\mathrm{C}$ & 0.1439 & -1.2931 & 0.326 & & & & \\
\hline $\mathrm{C}$ & 1.1247 & -1.3354 & 1.5238 & $\mathrm{C}$ & 1.183 & -1.5298 & 1.4286 & & & & \\
\hline $\mathrm{C}$ & -0.7722 & 0.6346 & -1.3008 & $\mathrm{C}$ & -0.7481 & 0.5217 & -1.3174 & & & & \\
\hline $\mathrm{C}$ & -2.0979 & 0.4914 & -0.6296 & $\mathrm{C}$ & -2.0444 & 0.4631 & -0.5795 & & & & \\
\hline $\mathrm{C}$ & -2.394 & -0.8225 & 0.0199 & $\mathrm{C}$ & -2.3777 & -0.816 & 0.1208 & & & & \\
\hline $\mathrm{C}$ & -1.29 & -1.1855 & 1.009 & $\mathrm{C}$ & -1.2409 & -1.2374 & 1.0483 & & & & \\
\hline $\mathrm{C}$ & -3.0679 & 1.4 & -0.4446 & $\mathrm{C}$ & -2.9514 & 1.4295 & -0.3699 & & & & \\
\hline $\mathrm{C}$ & -4.0993 & 0.7312 & 0.3746 & $\mathrm{C}$ & -3.9654 & 0.8461 & 0.5318 & & & & \\
\hline $\mathrm{N}$ & -3.6471 & -0.537 & 0.6414 & $\mathrm{~N}$ & -3.568 & -0.4377 & 0.8138 & & & & \\
\hline $\mathrm{C}$ & 0.2383 & -2.3095 & -0.664 & $\mathrm{C}$ & 0.1235 & -2.4498 & -0.7046 & & & & \\
\hline $\mathrm{C}$ & -3.2046 & 2.8141 & -0.8566 & $\mathrm{C}$ & -3.0415 & 2.8305 & -0.8368 & & & & \\
\hline $\mathrm{C}$ & 2.8115 & 0.9863 & -0.0528 & $\mathrm{C}$ & 2.9702 & 0.63 & -0.2399 & & & & \\
\hline $\mathrm{O}$ & 4.0564 & 0.6616 & -0.4881 & $\mathrm{O}$ & 2.5778 & 1.4215 & 0.7899 & & & & \\
\hline $\mathrm{O}$ & 2.5605 & 1.6404 & 0.9488 & $\mathrm{O}$ & 4.1249 & 0.3284 & -0.5035 & & & & \\
\hline $\mathrm{C}$ & 5.1076 & 1.162 & 0.3377 & $\mathrm{C}$ & 3.6445 & 1.8847 & 1.6177 & & & & \\
\hline $\mathrm{O}$ & -5.1491 & 1.219 & 0.745 & $\mathrm{O}$ & -4.9586 & 1.4059 & 0.9518 & & & & \\
\hline $\mathrm{H}$ & -2.5547 & -1.6109 & -0.7224 & $\mathrm{H}$ & -2.6362 & -1.6051 & -0.5927 & & & & \\
\hline $\mathrm{H}$ & 0.2937 & 1.0031 & 0.4812 & $\mathrm{H}$ & 0.3882 & 0.8578 & 0.4252 & & & & \\
\hline $\mathrm{H}$ & 2.643 & -2.3214 & 2.6007 & $\mathrm{H}$ & 2.7694 & -2.5601 & 2.3603 & & & & \\
\hline $\mathrm{H}$ & 2.0943 & -3.1813 & 1.0597 & $\mathrm{H}$ & 2.1855 & -3.2986 & 0.7731 & & & & \\
\hline $\mathrm{H}$ & 3.0517 & 0.3066 & -2.6437 & $\mathrm{H}$ & 3.0387 & 0.0538 & -2.7598 & & & & \\
\hline $\mathrm{H}$ & 1.3292 & -0.2627 & -2.9279 & $\mathrm{H}$ & 1.2685 & -0.3639 & -3.0415 & & & & \\
\hline $\mathrm{H}$ & 1.1166 & -0.5342 & 2.2671 & $\mathrm{H}$ & 1.1847 & -0.7913 & 2.2336 & & & & \\
\hline $\mathrm{H}$ & -0.6198 & 1.6611 & -1.6541 & $\mathrm{H}$ & -0.5607 & 1.5326 & -1.6977 & & & & \\
\hline $\mathrm{H}$ & -0.7716 & -0.0061 & -2.1894 & $\mathrm{H}$ & -0.8174 & -0.1351 & -2.1917 & & & & \\
\hline $\mathrm{H}$ & -1.3225 & -0.4871 & 1.8592 & $\mathrm{H}$ & -1.1864 & -0.5296 & 1.8898 & & & & \\
\hline $\mathrm{H}$ & -1.4997 & -2.1756 & 1.4368 & $\mathrm{H}$ & -1.4815 & -2.2103 & 1.5001 & & & & \\
\hline $\mathrm{H}$ & -4.2108 & -1.1876 & 1.1709 & $\mathrm{H}$ & -4.1316 & -1.0385 & 1.4001 & & & & \\
\hline $\mathrm{H}$ & -0.4355 & -2.1688 & -1.5142 & $\mathrm{H}$ & -0.6099 & -2.2891 & -1.4999 & & & & \\
\hline $\mathrm{H}$ & 1.251 & -2.4072 & -1.0701 & $\mathrm{H}$ & 1.0953 & -2.5847 & -1.1916 & & & & \\
\hline $\mathrm{H}$ & -0.0237 & -3.2721 & -0.2082 & $\mathrm{H}$ & -0.1356 & -3.4012 & -0.2232 & & & & \\
\hline $\mathrm{H}$ & -3.3072 & 3.4594 & 0.0216 & $\mathrm{H}$ & -2.8906 & 3.523 & -0.0027 & & & & \\
\hline $\mathrm{H}$ & -4.0908 & 2.9451 & -1.4859 & $\mathrm{H}$ & -4.0267 & 3.0265 & -1.2724 & & & & \\
\hline
\end{tabular}




\begin{tabular}{|c|c|c|c|c|c|c|c|}
\hline $\mathrm{H}$ & -2.338 & 3.1618 & -1.4256 & $\mathrm{H}$ & -2.2938 & 3.0555 & -1.6032 \\
\hline $\mathrm{H}$ & 5.0338 & 0.7478 & 1.3482 & $\mathrm{H}$ & 4.2397 & 2.632 & 1.0834 \\
\hline $\mathrm{H}$ & 5.086 & 2.256 & 0.3635 & $\mathrm{H}$ & 4.2737 & 1.0538 & 1.9542 \\
\hline $\mathrm{H}$ & 6.0601 & 0.8463 & -0.0973 & $\mathrm{H}$ & 3.2034 & 2.3587 & 2.5 \\
\hline
\end{tabular}

Table S19. Stable conformers of compound $\mathbf{8}$ with $(1 R, 6 R)-8 \mathbf{a}$ and $(1 S, 6 S)-8 \mathbf{b}$ configurations, respectively.

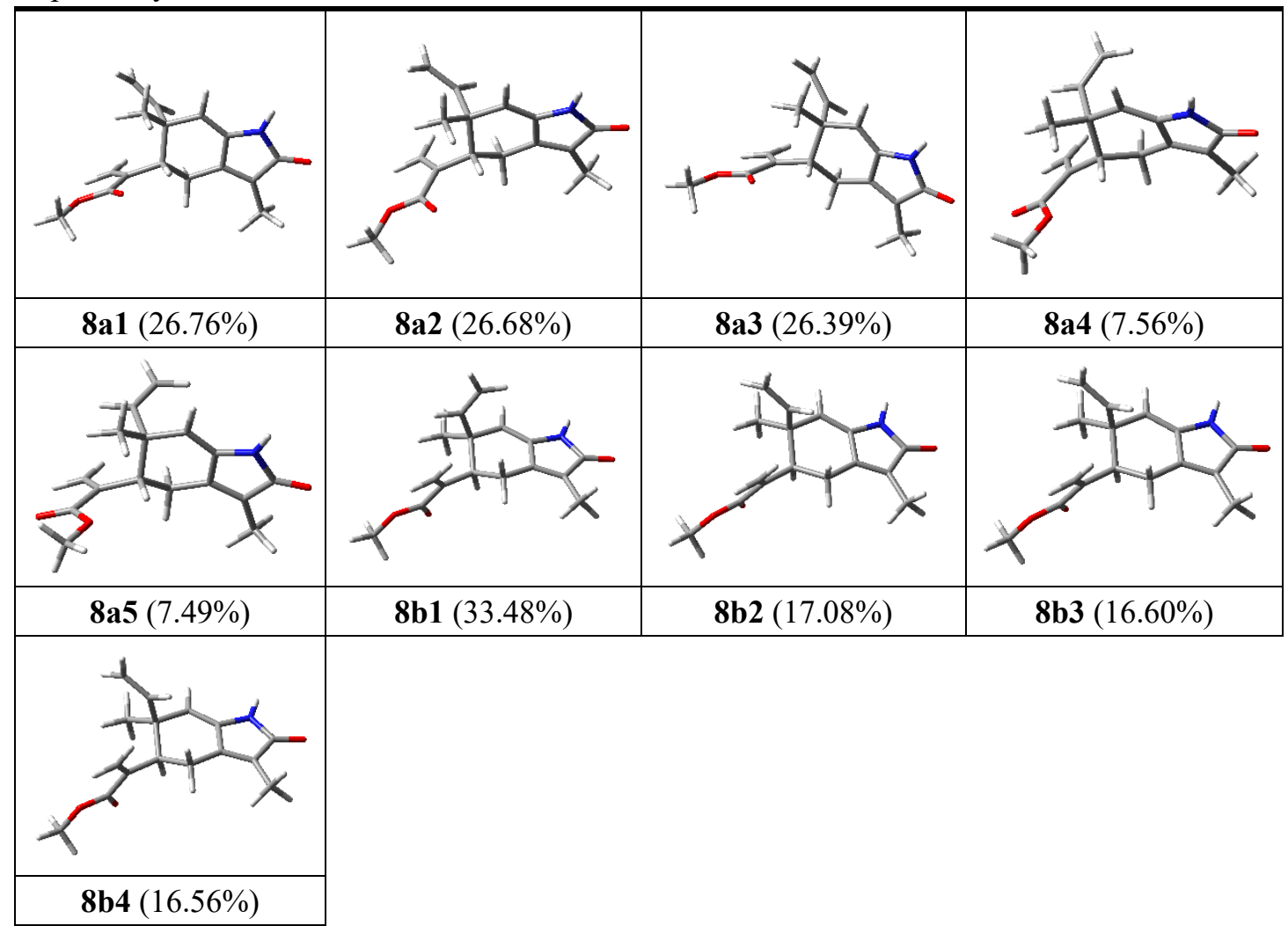

Table S20. Important thermodynamic parameters (a.u.) of the optimized compound 8 with structures at B3LYP/6-31+G(d,p) level in the gas phase.

\begin{tabular}{c|c|c|c|c|c}
\hline conformations & E + ZPE & G & conformations & E+ZPE & G \\
\hline $\mathbf{8 a 1}$ & -901.242612 & -901.292499 & $\mathbf{8 b 1}$ & -901.242614 & -901.292509 \\
\hline $\mathbf{8 a 2}$ & -901.242611 & -901.292496 & $\mathbf{8 b 2}$ & -901.243680 & -901.293144 \\
\hline $\mathbf{8 a 3}$ & -901.242611 & -901.292486 & $\mathbf{8 b 3}$ & -901.242609 & -901.292480 \\
\hline $\mathbf{8 a 4}$ & -901.242337 & -901.291306 & $\mathbf{8 b 4}$ & -901.242610 & -901.292482 \\
\hline $\mathbf{8 a 5}$ & -901.242335 & -901.291298 & & & \\
\hline
\end{tabular}

Table S21. Optimized Z-Matrixes of compound 8 with structures in the Gas Phase $(\AA)$ at B3LYP/6$31+\mathrm{G}(\mathrm{d}, \mathrm{p})$ level.

\begin{tabular}{|l|l|l|l|l|l|l|l|l|l|l|l|}
\hline \multicolumn{3}{|c|}{ 8a1 } & \multicolumn{6}{c|}{ 8a2 } & \multicolumn{4}{c|}{ 8a3 } \\
\hline C & -1.6474 & -2.9054 & -1.3553 & C & -1.6859 & -2.8537 & -1.5486 & C & -2.2948 & -3.0709 & -0.9015 \\
\hline C & 1.6746 & -1.2136 & -2.3573 & C & 1.6509 & -1.1495 & -2.3795 & C & 1.4003 & -2.6307 & -1.1391 \\
\hline C & 1.59 & -1.0146 & -1.0276 & C & 1.5995 & -0.9679 & -1.0464 & C & 1.5107 & -1.4178 & -0.5686 \\
\hline C & 0.5308 & -0.139 & -0.3489 & C & 0.5073 & -0.1681 & -0.3314 & C & 0.3713 & -0.533 & -0.048 \\
\hline C & -0.8011 & -0.9159 & 0.0573 & C & -0.8241 & -0.9793 & 0.0061 & C & -1.0401 & -1.1818 & 0.3342 \\
\hline
\end{tabular}




\begin{tabular}{|c|c|c|c|c|c|c|c|c|c|c|c|}
\hline $\mathrm{C}$ & -1.444 & -1.5955 & -1.1536 & $\mathrm{C}$ & -1.4674 & -1.5634 & -1.2545 & $\mathrm{C}$ & -1.749 & -1.8465 & -0.8488 \\
\hline C & 0.2331 & 1.1664 & -1.1418 & $\mathrm{C}$ & 0.2086 & 1.1806 & -1.0447 & $\mathrm{C}$ & 0.2003 & 0.6992 & -0.9844 \\
\hline $\mathrm{C}$ & -0.7603 & 2.0172 & -0.4196 & $\mathrm{C}$ & -0.7882 & 1.9775 & -0.2691 & $\mathrm{C}$ & -0.7845 & 1.6723 & -0.4215 \\
\hline C & -1.7503 & 1.3755 & 0.3971 & $\mathrm{C}$ & -1.7711 & 1.2818 & 0.5108 & $\mathrm{C}$ & -1.8187 & 1.1927 & 0.4502 \\
\hline C & -1.8312 & 0.0655 & 0.6235 & $\mathrm{C}$ & -1.8526 & -0.0411 & 0.6463 & $\mathrm{C}$ & -1.9858 & -0.0761 & 0.8195 \\
\hline $\mathrm{C}$ & -0.924 & 3.3504 & -0.3881 & $\mathrm{C}$ & -0.948 & 3.3057 & -0.1463 & $\mathrm{C}$ & -0.8804 & 3.0044 & -0.5667 \\
\hline $\mathrm{C}$ & -2.1192 & 3.595 & 0.4686 & $\mathrm{C}$ & -2.1314 & 3.4933 & 0.7408 & $\mathrm{C}$ & -2.0671 & 3.4193 & 0.2325 \\
\hline $\mathrm{N}$ & -2.5491 & 2.3528 & 0.8833 & $\mathrm{~N}$ & -2.5601 & 2.2257 & 1.0732 & $\mathrm{~N}$ & -2.5626 & 2.2663 & 0.8026 \\
\hline $\mathrm{C}$ & -0.4768 & -1.9131 & 1.1965 & $\mathrm{C}$ & -0.4995 & -2.0644 & 1.0601 & $\mathrm{C}$ & -0.819 & -2.1419 & 1.5282 \\
\hline $\mathrm{C}$ & -0.1871 & 4.4544 & -1.0368 & $\mathrm{C}$ & -0.2099 & 4.4459 & -0.726 & $\mathrm{C}$ & -0.0725 & 3.975 & -1.3335 \\
\hline $\mathrm{C}$ & 2.6101 & -1.6226 & -0.1134 & $\mathrm{C}$ & 2.6844 & -1.5145 & -0.1665 & $\mathrm{C}$ & 2.8834 & -0.8417 & -0.3542 \\
\hline $\mathrm{O}$ & 3.3046 & -2.6132 & -0.7291 & $\mathrm{O}$ & 3.6633 & -2.1109 & -0.8937 & $\mathrm{O}$ & 3.8048 & -1.4326 & -1.1573 \\
\hline $\mathrm{O}$ & 2.7592 & -1.2772 & 1.0495 & $\mathrm{O}$ & 2.6755 & -1.4166 & 1.0521 & $\mathrm{O}$ & 3.1108 & 0.0769 & 0.4195 \\
\hline $\mathrm{C}$ & 4.2664 & -3.256 & 0.1078 & $\mathrm{C}$ & 4.738 & -2.6325 & -0.1123 & $\mathrm{C}$ & 5.1229 & -0.8993 & -1.0238 \\
\hline $\mathrm{O}$ & -2.6049 & 4.6769 & 0.735 & $\mathrm{O}$ & -2.6077 & 4.5555 & 1.0899 & $\mathrm{O}$ & -2.499 & 4.5503 & 0.3456 \\
\hline $\mathrm{H}$ & 0.9734 & 0.2258 & 0.5919 & $\mathrm{H}$ & 0.9277 & 0.1421 & 0.6388 & $\mathrm{H}$ & 0.7407 & -0.1296 & 0.9104 \\
\hline $\mathrm{H}$ & -2.1199 & -3.2532 & -2.2697 & $\mathrm{H}$ & -2.1584 & -3.1319 & -2.4863 & $\mathrm{H}$ & -2.2758 & -3.7615 & -0.0656 \\
\hline $\mathrm{H}$ & -1.3559 & -3.6659 & -0.6389 & $\mathrm{H}$ & -1.4108 & -3.6649 & -0.8839 & $\mathrm{H}$ & -2.7915 & -3.4114 & -1.8064 \\
\hline $\mathrm{H}$ & 2.4531 & -1.8232 & -2.8077 & $\mathrm{H}$ & 2.449 & -1.7054 & -2.8639 & $\mathrm{H}$ & 0.4506 & -3.1164 & -1.314 \\
\hline $\mathrm{H}$ & 0.9565 & -0.7901 & -3.0506 & $\mathrm{H}$ & 0.8843 & -0.763 & -3.0417 & $\mathrm{H}$ & 2.2664 & -3.2042 & -1.459 \\
\hline $\mathrm{H}$ & -1.7778 & -0.9229 & -1.9447 & $\mathrm{H}$ & -1.7924 & -0.8321 & -1.9958 & $\mathrm{H}$ & -1.8483 & -1.2318 & -1.7444 \\
\hline $\mathrm{H}$ & -0.1772 & 0.9351 & -2.1319 & $\mathrm{H}$ & -0.1951 & 1.0111 & -2.0501 & $\mathrm{H}$ & 1.1633 & 1.2024 & -1.1328 \\
\hline $\mathrm{H}$ & 1.1659 & 1.7214 & -1.2996 & $\mathrm{H}$ & 1.1388 & 1.75 & -1.1619 & $\mathrm{H}$ & -0.1387 & 0.3724 & -1.9752 \\
\hline $\mathrm{H}$ & -2.6387 & -0.3347 & 1.2307 & $\mathrm{H}$ & -2.6533 & -0.4819 & 1.2329 & $\mathrm{H}$ & -2.822 & -0.3556 & 1.4544 \\
\hline $\mathrm{H}$ & -3.3446 & 2.2259 & 1.4898 & $\mathrm{H}$ & -3.3452 & 2.0587 & 1.6829 & $\mathrm{H}$ & -3.3704 & 2.2611 & 1.4057 \\
\hline $\mathrm{H}$ & -1.3802 & -2.4164 & 1.5635 & $\mathrm{H}$ & -0.0548 & -1.6242 & 1.9611 & $\mathrm{H}$ & -0.3214 & -1.6304 & 2.3616 \\
\hline $\mathrm{H}$ & -0.0268 & -1.4004 & 2.0554 & $\mathrm{H}$ & -1.4014 & -2.6016 & 1.3811 & $\mathrm{H}$ & -1.766 & -2.5341 & 1.9194 \\
\hline $\mathrm{H}$ & 0.2199 & -2.6948 & 0.8766 & $\mathrm{H}$ & 0.2038 & -2.8117 & 0.6763 & $\mathrm{H}$ & -0.1976 & -3.001 & 1.2544 \\
\hline $\mathrm{H}$ & 0.6411 & 4.0854 & -1.6493 & $\mathrm{H}$ & 0.2597 & 5.0387 & 0.0652 & $\mathrm{H}$ & 0.3952 & 4.6988 & -0.6588 \\
\hline $\mathrm{H}$ & -0.8527 & 5.0313 & -1.6864 & $\mathrm{H}$ & 0.5777 & 4.1141 & -1.4088 & $\mathrm{H}$ & 0.7215 & 3.4816 & -1.9021 \\
\hline $\mathrm{H}$ & 0.2294 & 5.13 & -0.283 & $\mathrm{H}$ & -0.8858 & 5.0956 & -1.2901 & $\mathrm{H}$ & -0.7005 & 4.5216 & -2.044 \\
\hline $\mathrm{H}$ & 3.7719 & -3.7411 & 0.9552 & $\mathrm{H}$ & 5.4619 & -3.0901 & -0.7925 & $\mathrm{H}$ & 5.4971 & -1.0537 & -0.0069 \\
\hline $\mathrm{H}$ & 5.0161 & -2.5378 & 0.4544 & $\mathrm{H}$ & 5.239 & -1.828 & 0.4352 & $\mathrm{H}$ & 5.136 & 0.1635 & -1.2854 \\
\hline $\mathrm{H}$ & 4.7708 & -4.0243 & -0.4841 & $\mathrm{H}$ & 4.3746 & -3.4037 & 0.574 & $\mathrm{H}$ & 5.7764 & -1.4341 & -1.7185 \\
\hline \multicolumn{4}{|c|}{$8 \mathrm{a} 4$} & \multicolumn{4}{|c|}{$8 a 5$} & \multicolumn{4}{|c|}{$8 b 1$} \\
\hline $\mathrm{C}$ & -2.7089 & -1.5467 & -1.3735 & $\mathrm{C}$ & -2.6848 & -1.8721 & -0.7693 & $\mathrm{C}$ & 1.0593 & 2.3858 & -2.0786 \\
\hline $\mathrm{C}$ & 1.4205 & -1.3732 & -2.6855 & $\mathrm{C}$ & 1.2667 & -1.7032 & -2.5185 & $\mathrm{C}$ & 1.996 & -1.7371 & -1.2111 \\
\hline $\mathrm{C}$ & 1.5125 & -1.2168 & -1.3505 & $\mathrm{C}$ & 1.476 & -1.2923 & -1.2536 & $\mathrm{C}$ & 1.5134 & -1.3153 & -0.0262 \\
\hline $\mathrm{C}$ & 0.5862 & -0.3348 & -0.5102 & $\mathrm{C}$ & 0.5687 & -0.3277 & -0.4891 & $\mathrm{C}$ & 0.3048 & -0.3844 & 0.1263 \\
\hline $\mathrm{C}$ & -0.6597 & -1.0916 & 0.1351 & $\mathrm{C}$ & -0.545 & -1.0275 & 0.4107 & $\mathrm{C}$ & 0.6355 & 1.1732 & 0.1663 \\
\hline $\mathrm{C}$ & -1.4775 & -1.8352 & -0.9256 & $\mathrm{C}$ & -1.4012 & -2.0027 & -0.4021 & $\mathrm{C}$ & 1.4484 & 1.6091 & -1.0563 \\
\hline $\mathrm{C}$ & 0.1311 & 0.9508 & -1.266 & $\mathrm{C}$ & -0.0528 & 0.7708 & -1.4047 & $\mathrm{C}$ & -0.8202 & -0.6939 & -0.9059 \\
\hline $\mathrm{C}$ & -0.7012 & 1.8253 & -0.3836 & $\mathrm{C}$ & -0.8431 & 1.7471 & -0.595 & $\mathrm{C}$ & -2.0255 & 0.1482 & -0.6428 \\
\hline
\end{tabular}




\begin{tabular}{|c|c|c|c|c|c|c|c|c|c|c|c|}
\hline $\mathrm{C}$ & -1.4928 & 1.2115 & 0.6426 & $\mathrm{C}$ & -1.4835 & 1.2832 & 0.6027 & $\mathrm{C}$ & -1.8398 & 1.4514 & -0.0718 \\
\hline $\mathrm{C}$ & -1.5292 & -0.0937 & 0.9069 & $\mathrm{C}$ & -1.4062 & 0.0443 & 1.0852 & $\mathrm{C}$ & -0.6673 & 1.9715 & 0.288 \\
\hline $\mathrm{C}$ & -0.8712 & 3.1574 & -0.3678 & $\mathrm{C}$ & -1.0938 & 3.0537 & -0.7809 & $\mathrm{C}$ & -3.3303 & -0.0884 & -0.8563 \\
\hline $\mathrm{C}$ & -1.8556 & 3.4314 & 0.717 & $\mathrm{C}$ & -1.9708 & 3.4659 & 0.3515 & $\mathrm{C}$ & -4.0432 & 1.1485 & -0.4273 \\
\hline $\mathrm{N}$ & -2.1748 & 2.2044 & 1.2577 & $\mathrm{~N}$ & -2.1568 & 2.3362 & 1.119 & $\mathrm{~N}$ & -3.0668 & 2.0143 & 0.0206 \\
\hline $\mathrm{C}$ & -0.1743 & -2.1401 & 1.1617 & $\mathrm{C}$ & 0.1198 & -1.8353 & 1.5483 & $\mathrm{C}$ & 1.4759 & 1.5044 & 1.4197 \\
\hline $\mathrm{C}$ & -0.3074 & 4.2343 & -1.2063 & $\mathrm{C}$ & -0.6967 & 3.9971 & -1.8467 & $\mathrm{C}$ & -4.0419 & -1.2539 & -1.421 \\
\hline $\mathrm{C}$ & 2.6361 & -1.9635 & -0.7 & $\mathrm{C}$ & 2.7077 & -1.8341 & -0.5996 & $\mathrm{C}$ & 2.1178 & -1.8083 & 1.2555 \\
\hline $\mathrm{O}$ & 2.8885 & -1.4671 & 0.5362 & $\mathrm{O}$ & 3.0713 & -1.0533 & 0.4464 & $\mathrm{O}$ & 3.2084 & -2.5842 & 1.0306 \\
\hline $\mathrm{O}$ & 3.2394 & -2.8885 & -1.2243 & $\mathrm{O}$ & 3.2944 & -2.8415 & -0.9673 & $\mathrm{O}$ & 1.6743 & -1.5257 & 2.3593 \\
\hline $\mathrm{C}$ & 3.9512 & -2.1271 & 1.2264 & $\mathrm{C}$ & 4.2299 & -1.5114 & 1.1451 & $\mathrm{C}$ & 3.8247 & -3.0806 & 2.2187 \\
\hline $\mathrm{O}$ & -2.2817 & 4.5218 & 1.0444 & $\mathrm{O}$ & -2.4209 & 4.5784 & 0.5441 & $\mathrm{O}$ & -5.2423 & 1.3413 & -0.4737 \\
\hline $\mathrm{H}$ & 1.1827 & 0.057 & 0.3277 & $\mathrm{H}$ & 1.2082 & 0.2426 & 0.2014 & $\mathrm{H}$ & -0.1544 & -0.6288 & 1.098 \\
\hline $\mathrm{H}$ & -3.2837 & -0.7019 & -1.0088 & $\mathrm{H}$ & -3.284 & -1.004 & -0.5159 & $\mathrm{H}$ & 1.7477 & 2.617 & -2.8862 \\
\hline $\mathrm{H}$ & -3.1752 & -2.161 & -2.138 & $\mathrm{H}$ & -3.1723 & -2.6479 & -1.353 & $\mathrm{H}$ & 0.0627 & 2.8083 & -2.1512 \\
\hline $\mathrm{H}$ & 0.6486 & -0.8958 & -3.2782 & $\mathrm{H}$ & 0.4035 & -1.3902 & -3.0946 & $\mathrm{H}$ & 2.8375 & -2.4197 & -1.2903 \\
\hline $\mathrm{H}$ & 2.1098 & -2.0093 & -3.2368 & $\mathrm{H}$ & 1.9477 & -2.3888 & -3.0179 & $\mathrm{H}$ & 1.5743 & -1.4061 & -2.1541 \\
\hline $\mathrm{H}$ & -0.9889 & -2.7038 & -1.369 & $\mathrm{H}$ & -0.8948 & -2.915 & -0.7221 & $\mathrm{H}$ & 2.4724 & 1.2343 & -1.0915 \\
\hline $\mathrm{H}$ & -0.4689 & 0.6923 & -2.1471 & $\mathrm{H}$ & 0.7438 & 1.2909 & -1.9516 & $\mathrm{H}$ & -0.4764 & -0.4866 & -1.9264 \\
\hline $\mathrm{H}$ & 1.012 & 1.5012 & -1.6197 & $\mathrm{H}$ & -0.7234 & 0.3223 & -2.1473 & $\mathrm{H}$ & -1.0832 & -1.758 & -0.8615 \\
\hline $\mathrm{H}$ & -2.192 & -0.4772 & 1.6776 & $\mathrm{H}$ & -1.9622 & -0.2297 & 1.9773 & $\mathrm{H}$ & -0.614 & 2.9833 & 0.6798 \\
\hline $\mathrm{H}$ & -2.8264 & 2.0986 & 2.0199 & $\mathrm{H}$ & -2.7107 & 2.3353 & 1.9608 & $\mathrm{H}$ & -3.2764 & 2.9325 & 0.3808 \\
\hline $\mathrm{H}$ & -1.0181 & -2.6334 & 1.6613 & $\mathrm{H}$ & -0.6264 & -2.2599 & 2.2318 & $\mathrm{H}$ & 0.9626 & 1.1909 & 2.3363 \\
\hline $\mathrm{H}$ & 0.417 & -2.9341 & 0.6936 & $\mathrm{H}$ & 0.7864 & -1.2038 & 2.1474 & $\mathrm{H}$ & 1.6648 & 2.5822 & 1.5046 \\
\hline $\mathrm{H}$ & 0.4404 & -1.6788 & 1.9437 & $\mathrm{H}$ & 0.709 & -2.6764 & 1.1681 & $\mathrm{H}$ & 2.4565 & 1.016 & 1.3982 \\
\hline $\mathrm{H}$ & 0.2626 & 4.9401 & -0.5939 & $\mathrm{H}$ & -0.1282 & 3.5001 & -2.6385 & $\mathrm{H}$ & -4.7693 & -1.6458 & -0.7035 \\
\hline $\mathrm{H}$ & -1.1072 & 4.7834 & -1.7132 & $\mathrm{H}$ & -0.0742 & 4.7984 & -1.4367 & $\mathrm{H}$ & -3.3538 & -2.0652 & -1.6761 \\
\hline $\mathrm{H}$ & 0.3634 & 3.8368 & -1.973 & $\mathrm{H}$ & -1.5807 & 4.4508 & -2.3074 & $\mathrm{H}$ & -4.576 & -0.9702 & -2.3327 \\
\hline $\mathrm{H}$ & 3.9702 & -1.7544 & 2.2534 & $\mathrm{H}$ & 4.4191 & -0.8223 & 1.9725 & $\mathrm{H}$ & 4.1673 & -2.2542 & 2.8495 \\
\hline $\mathrm{H}$ & 3.7891 & -3.209 & 1.2568 & $\mathrm{H}$ & 4.0569 & -2.5088 & 1.5601 & $\mathrm{H}$ & 4.6934 & -3.674 & 1.9221 \\
\hline $\mathrm{H}$ & 4.9083 & -1.8914 & 0.7529 & $\mathrm{H}$ & 5.1026 & -1.5091 & 0.4854 & $\mathrm{H}$ & 3.1327 & -3.7276 & 2.7672 \\
\hline \multicolumn{4}{|c|}{$8 \mathrm{~b} 2$} & \multicolumn{4}{|c|}{$8 b 3$} & \multicolumn{4}{|c|}{$8 b 4$} \\
\hline $\mathrm{C}$ & 2.4078 & 2.4257 & -1.0639 & $\mathrm{C}$ & 2.027 & 2.802 & -1.9298 & $\mathrm{C}$ & 2.4224 & 2.4259 & -1.5776 \\
\hline $\mathrm{C}$ & 2.1389 & -1.427 & -1.2613 & $\mathrm{C}$ & 2.9965 & -0.4626 & -0.4085 & $\mathrm{C}$ & 2.0821 & -1.4392 & -1.3428 \\
\hline $\mathrm{C}$ & 1.5749 & -1.1419 & -0.0715 & $\mathrm{C}$ & 1.8562 & -0.7493 & 0.245 & $\mathrm{C}$ & 1.5257 & -1.1011 & -0.1648 \\
\hline $\mathrm{C}$ & 0.2816 & -0.334 & 0.0945 & $\mathrm{C}$ & 0.5598 & 0.0714 & 0.2365 & $\mathrm{C}$ & 0.311 & -0.1877 & -0.0348 \\
\hline $\mathrm{C}$ & 0.4897 & 1.2432 & 0.1995 & $\mathrm{C}$ & 0.5989 & 1.6306 & -0.1242 & $\mathrm{C}$ & 0.5956 & 1.381 & -0.0808 \\
\hline $\mathrm{C}$ & 1.2252 & 1.795 & -1.0236 & $\mathrm{C}$ & 1.0926 & 1.9196 & -1.544 & $\mathrm{C}$ & 1.2336 & 1.8334 & -1.396 \\
\hline $\mathrm{C}$ & -0.7966 & -0.7167 & -0.9608 & $\mathrm{C}$ & -0.532 & -0.6767 & -0.5834 & $\mathrm{C}$ & -0.8463 & -0.6034 & -0.9843 \\
\hline $\mathrm{C}$ & -2.0622 & 0.0448 & -0.7372 & $\mathrm{C}$ & -1.8565 & 0.0063 & -0.4632 & $\mathrm{C}$ & -2.0673 & 0.1946 & -0.6584 \\
\hline $\mathrm{C}$ & -1.9868 & 1.3538 & -0.1545 & $\mathrm{C}$ & -1.892 & 1.4179 & -0.2063 & $\mathrm{C}$ & -1.9008 & 1.5414 & -0.1818 \\
\hline $\mathrm{C}$ & -0.8691 & 1.9483 & 0.2602 & $\mathrm{C}$ & -0.8236 & 2.1987 & -0.0529 & $\mathrm{C}$ & -0.732 & 2.1361 & 0.0624 \\
\hline $\mathrm{C}$ & -3.3399 & -0.2753 & -1.0007 & $\mathrm{C}$ & -3.1056 & -0.4839 & -0.5313 & $\mathrm{C}$ & -3.3691 & -0.1294 & -0.7169 \\
\hline
\end{tabular}




\begin{tabular}{|c|c|c|c|c|c|c|c|c|c|c|c|}
\hline $\mathrm{C}$ & -4.1485 & 0.9111 & -0.6011 & $\mathrm{C}$ & -4.0146 & 0.6811 & -0.3407 & $\mathrm{C}$ & -4.1075 & 1.0945 & -0.2988 \\
\hline $\mathrm{N}$ & -3.25 & 1.8364 & -0.1139 & $\mathrm{~N}$ & -3.1959 & 1.7743 & -0.1563 & $\mathrm{~N}$ & -3.1465 & 2.0425 & -0.0084 \\
\hline $\mathrm{C}$ & 1.2136 & 1.5693 & 1.5276 & $\mathrm{C}$ & 1.4268 & 2.3538 & 0.9656 & $\mathrm{C}$ & 1.4664 & 1.7599 & 1.1388 \\
\hline $\mathrm{C}$ & -3.9545 & -1.4803 & -1.5952 & $\mathrm{C}$ & -3.6192 & -1.8502 & -0.7598 & $\mathrm{C}$ & -4.0586 & -1.3683 & -1.1336 \\
\hline $\mathrm{C}$ & 2.177 & -1.6731 & 1.1956 & $\mathrm{C}$ & 1.8067 & -1.9685 & 1.1263 & $\mathrm{C}$ & 2.0352 & -1.6743 & 1.125 \\
\hline $\mathrm{O}$ & 3.3845 & -2.2528 & 0.9737 & $\mathrm{O}$ & 2.8597 & -2.7954 & 0.9024 & $\mathrm{O}$ & 2.8501 & -2.7402 & 0.922 \\
\hline $\mathrm{O}$ & 1.6377 & -1.5737 & 2.288 & $\mathrm{O}$ & 0.9007 & -2.185 & 1.9182 & $\mathrm{O}$ & 1.6988 & -1.2517 & 2.2215 \\
\hline $\mathrm{C}$ & 4.0103 & -2.7664 & 2.15 & $\mathrm{C}$ & 2.8495 & -3.9829 & 1.6955 & $\mathrm{C}$ & 3.2673 & -3.3886 & 2.1267 \\
\hline $\mathrm{O}$ & -5.355 & 1.0232 & -0.6971 & $\mathrm{O}$ & -5.2298 & 0.6561 & -0.3505 & $\mathrm{O}$ & -5.315 & 1.2122 & -0.2316 \\
\hline $\mathrm{H}$ & -0.1695 & -0.6485 & 1.0494 & $\mathrm{H}$ & 0.2097 & 0.0564 & 1.283 & $\mathrm{H}$ & -0.1026 & -0.3837 & 0.9667 \\
\hline $\mathrm{H}$ & 2.8137 & 2.7698 & -2.0113 & $\mathrm{H}$ & 2.5688 & 3.4308 & -1.2317 & $\mathrm{H}$ & 2.7451 & 2.7274 & -2.5695 \\
\hline $\mathrm{H}$ & 3.0052 & 2.6187 & -0.1795 & $\mathrm{H}$ & 2.2696 & 2.9193 & -2.9828 & $\mathrm{H}$ & 3.1114 & 2.6394 & -0.7657 \\
\hline $\mathrm{H}$ & 1.7272 & -1.0607 & -2.1953 & $\mathrm{H}$ & 3.1082 & 0.4007 & -1.0486 & $\mathrm{H}$ & 1.7008 & -1.0577 & -2.2837 \\
\hline $\mathrm{H}$ & 3.044 & -2.0206 & -1.3535 & $\mathrm{H}$ & 3.885 & -1.0836 & -0.3308 & $\mathrm{H}$ & 2.9384 & -2.1031 & -1.4196 \\
\hline $\mathrm{H}$ & 0.7124 & 1.6599 & -1.9766 & $\mathrm{H}$ & 0.5983 & 1.3584 & -2.3376 & $\mathrm{H}$ & 0.6267 & 1.6906 & -2.2892 \\
\hline $\mathrm{H}$ & -0.9891 & -1.7958 & -0.9129 & $\mathrm{H}$ & -0.6253 & -1.7138 & -0.2407 & $\mathrm{H}$ & -1.0554 & -1.6747 & -0.8804 \\
\hline $\mathrm{H}$ & -0.4454 & -0.4957 & -1.9755 & $\mathrm{H}$ & -0.2426 & -0.7205 & -1.6405 & $\mathrm{H}$ & -0.5786 & -0.4277 & -2.0331 \\
\hline $\mathrm{H}$ & -0.9024 & 2.9605 & 0.6541 & $\mathrm{H}$ & -0.944 & 3.2675 & 0.1006 & $\mathrm{H}$ & -0.6976 & 3.1717 & 0.3895 \\
\hline $\mathrm{H}$ & -3.5326 & 2.7385 & 0.2371 & $\mathrm{H}$ & -3.5549 & 2.7028 & 0.0031 & $\mathrm{H}$ & -3.3773 & 2.968 & 0.3161 \\
\hline $\mathrm{H}$ & 2.2224 & 1.1446 & 1.5626 & $\mathrm{H}$ & 1.3958 & 3.4438 & 0.8475 & $\mathrm{H}$ & 2.4662 & 1.3172 & 1.0778 \\
\hline $\mathrm{H}$ & 0.659 & 1.1756 & 2.3882 & $\mathrm{H}$ & 2.4793 & 2.052 & 0.9478 & $\mathrm{H}$ & 1.0074 & 1.419 & 2.0732 \\
\hline $\mathrm{H}$ & 1.3129 & 2.6515 & 1.6802 & $\mathrm{H}$ & 1.0394 & 2.1345 & 1.9683 & $\mathrm{H}$ & 1.5913 & 2.8464 & 1.2178 \\
\hline $\mathrm{H}$ & -3.2007 & -2.2156 & -1.8925 & $\mathrm{H}$ & -2.8076 & -2.5655 & -0.9228 & $\mathrm{H}$ & -3.3804 & -2.0462 & -1.6635 \\
\hline $\mathrm{H}$ & -4.5327 & -1.2167 & -2.4864 & $\mathrm{H}$ & -4.2668 & -1.8745 & -1.642 & $\mathrm{H}$ & -4.8901 & -1.1429 & -1.808 \\
\hline $\mathrm{H}$ & -4.6269 & -1.9595 & -0.8769 & $\mathrm{H}$ & -4.1998 & -2.1904 & 0.1034 & $\mathrm{H}$ & -4.4573 & -1.902 & -0.2644 \\
\hline $\mathrm{H}$ & 4.1921 & -1.9625 & 2.8703 & $\mathrm{H}$ & 3.7452 & -4.5605 & 1.4511 & $\mathrm{H}$ & 3.6186 & -2.666 & 2.8695 \\
\hline $\mathrm{H}$ & 4.9732 & -3.1969 & 1.8617 & $\mathrm{H}$ & 2.8783 & -3.7347 & 2.7611 & $\mathrm{H}$ & 4.0993 & -4.061 & 1.8913 \\
\hline $\mathrm{H}$ & 3.3975 & -3.5562 & 2.5956 & $\mathrm{H}$ & 1.9701 & -4.5912 & 1.4619 & $\mathrm{H}$ & 2.4415 & -3.9822 & 2.5324 \\
\hline
\end{tabular}




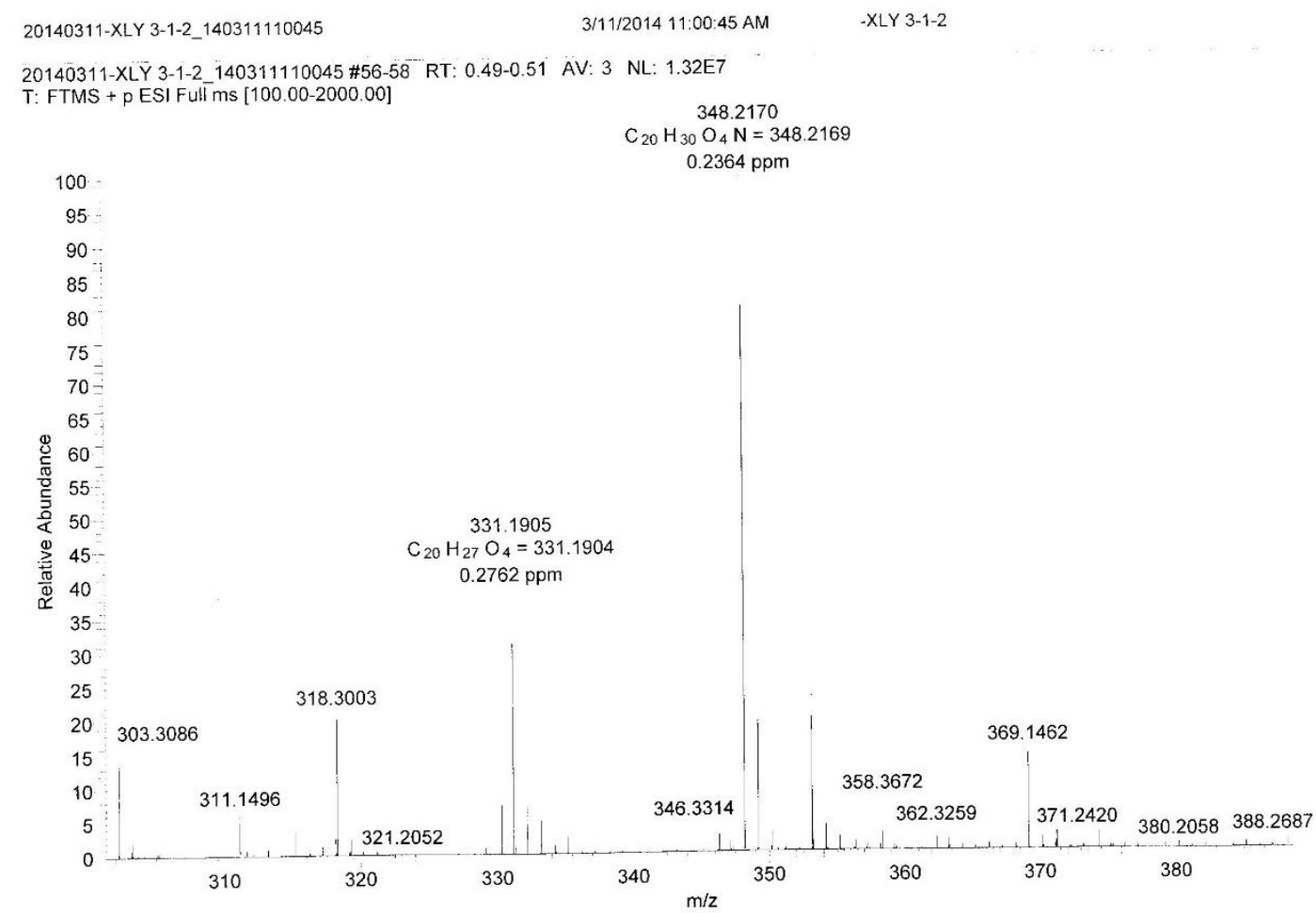

Figure S9. HRESIMS spectrum of compound 1.

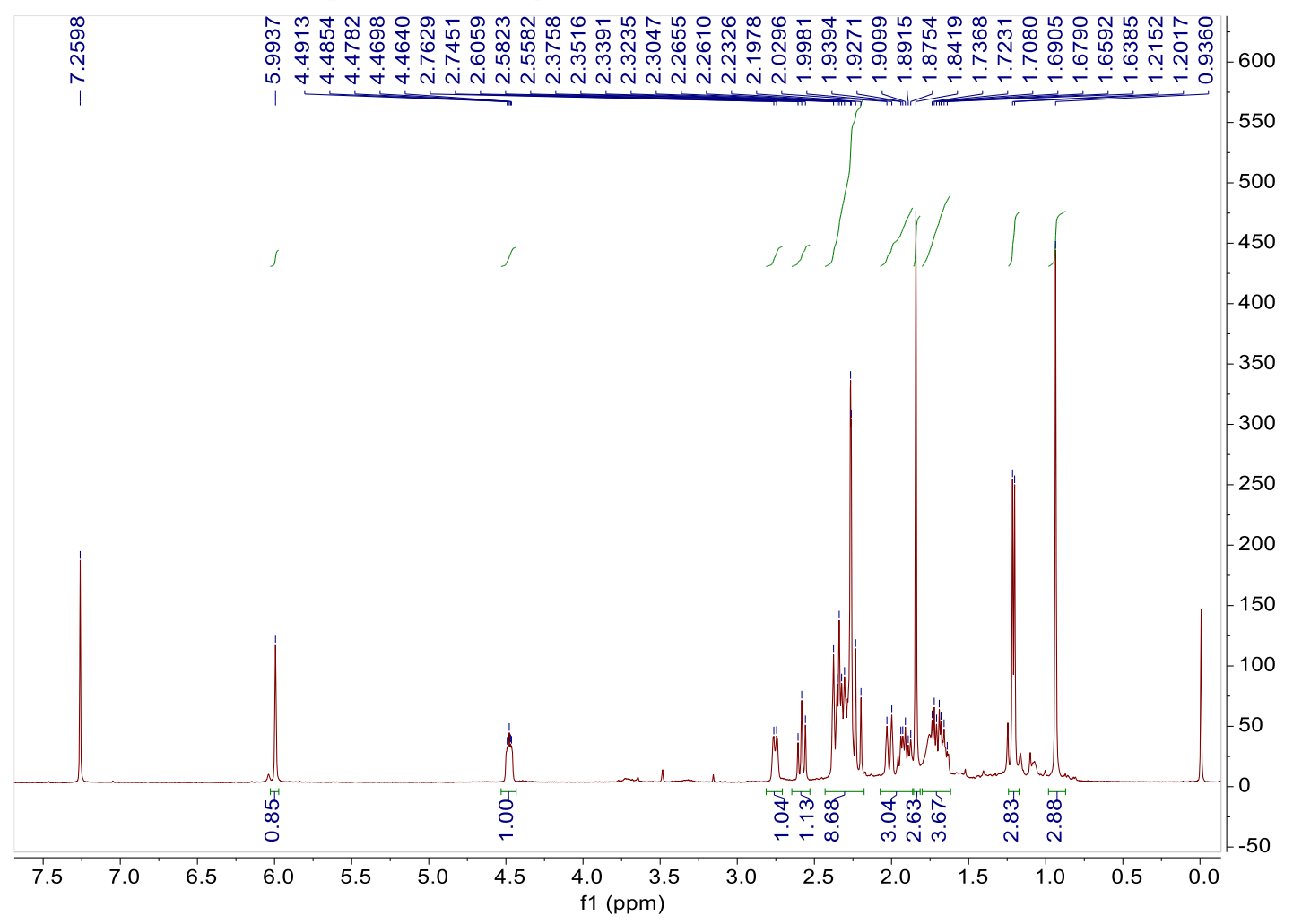

Figure S10. ${ }^{1} \mathrm{H}$ NMR spectrum of compound 1 in $\mathrm{CDCl}_{3}$. 


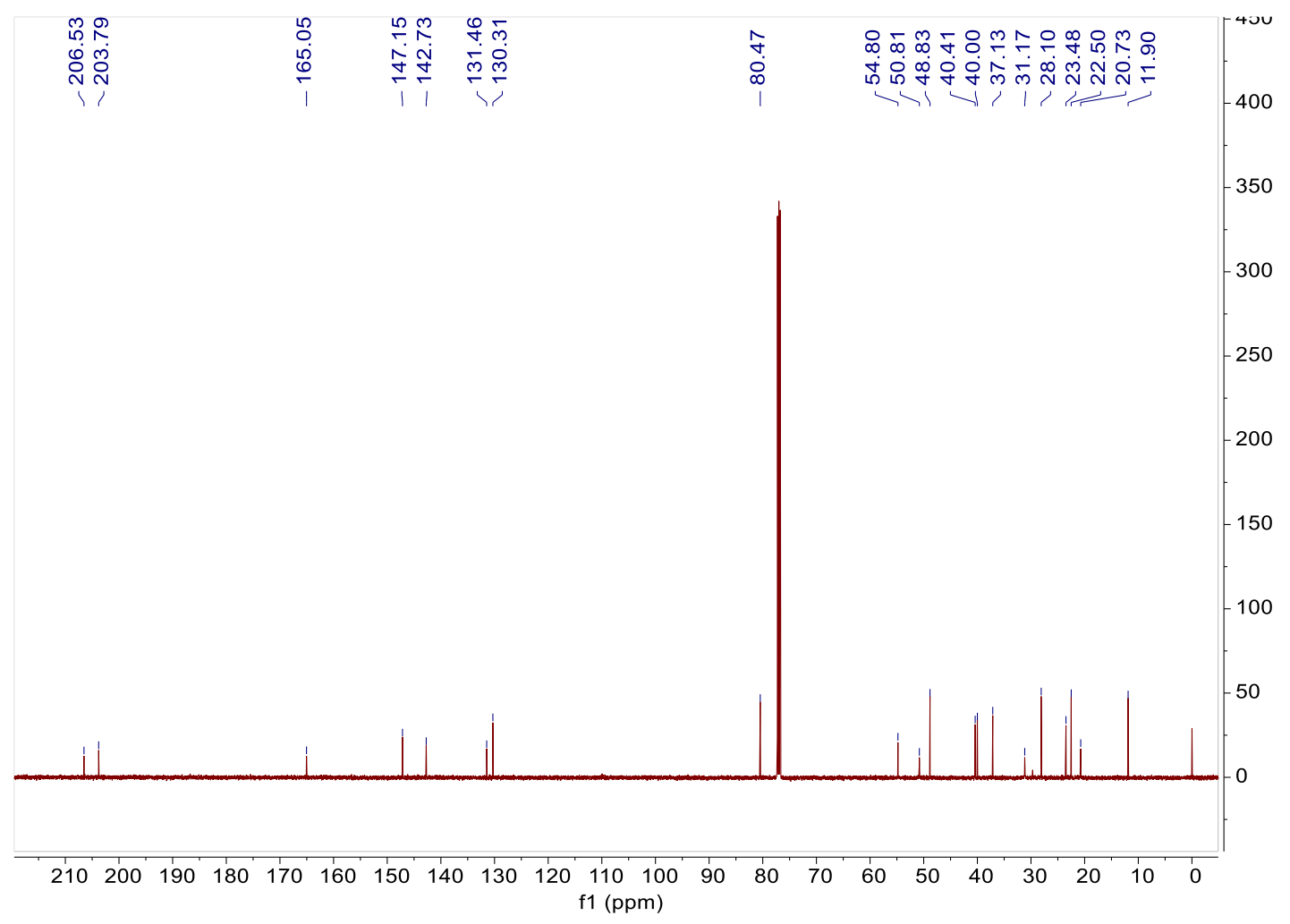

Figure S11. ${ }^{13} \mathrm{C}$ NMR spectrum of compound $\mathbf{1}$ in $\mathrm{CDCl}_{3}$.

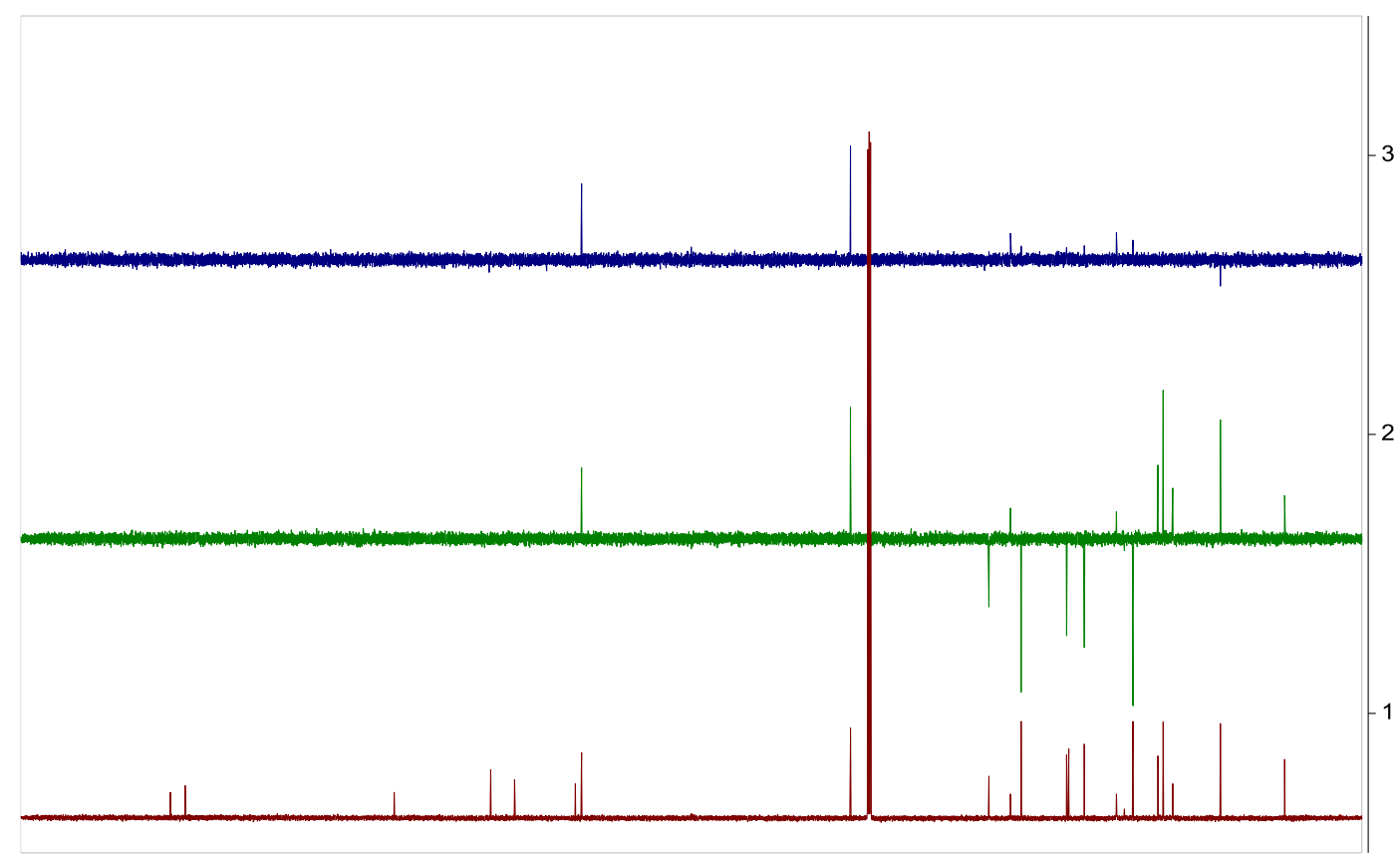

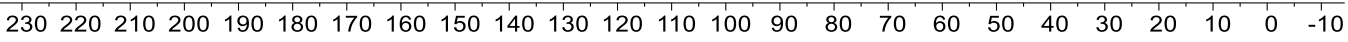
f1 (ppm)

Figure S12. ${ }^{13} \mathrm{C}$ and DEPT spectrum of compound 1 in $\mathrm{CDCl}_{3}$. 


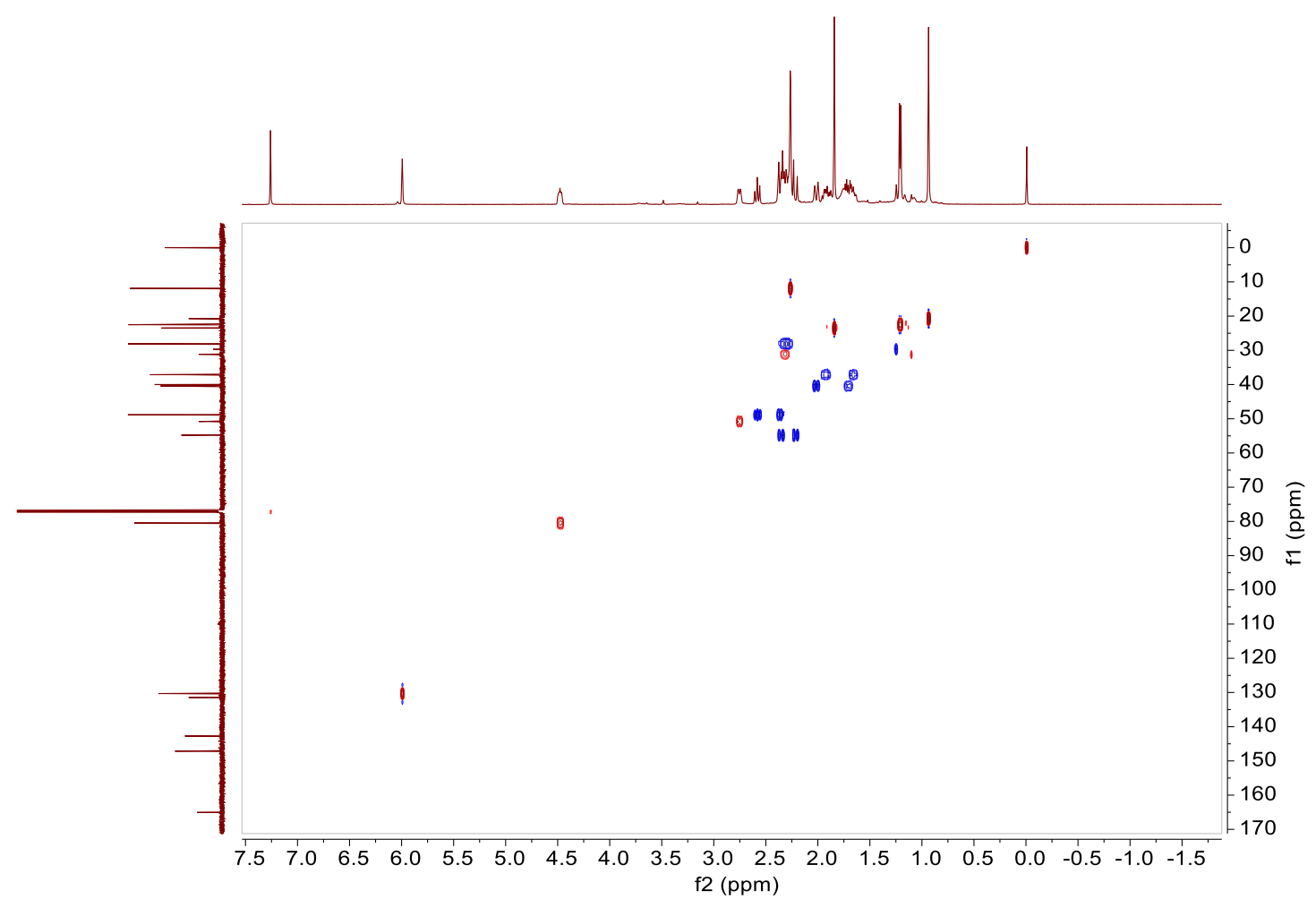

Figure S13. HSQC spectrum of compound $\mathbf{1}$ in $\mathrm{CDCl}_{3}$.

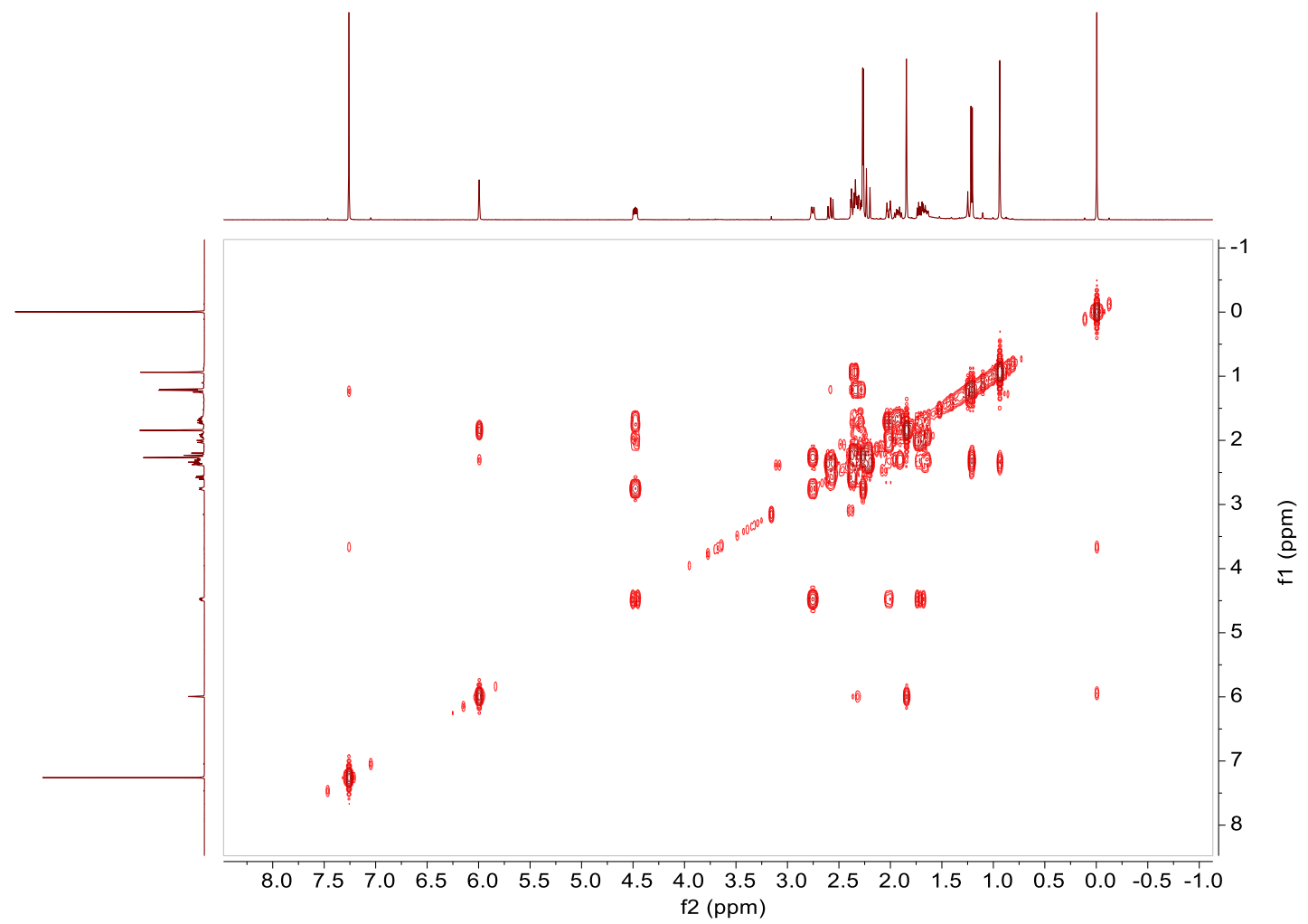

Figure S14. ${ }^{1} \mathrm{H}-{ }^{1} \mathrm{H}-\mathrm{COSY}$ spectrum of compound 1 in $\mathrm{CDCl}_{3}$. 


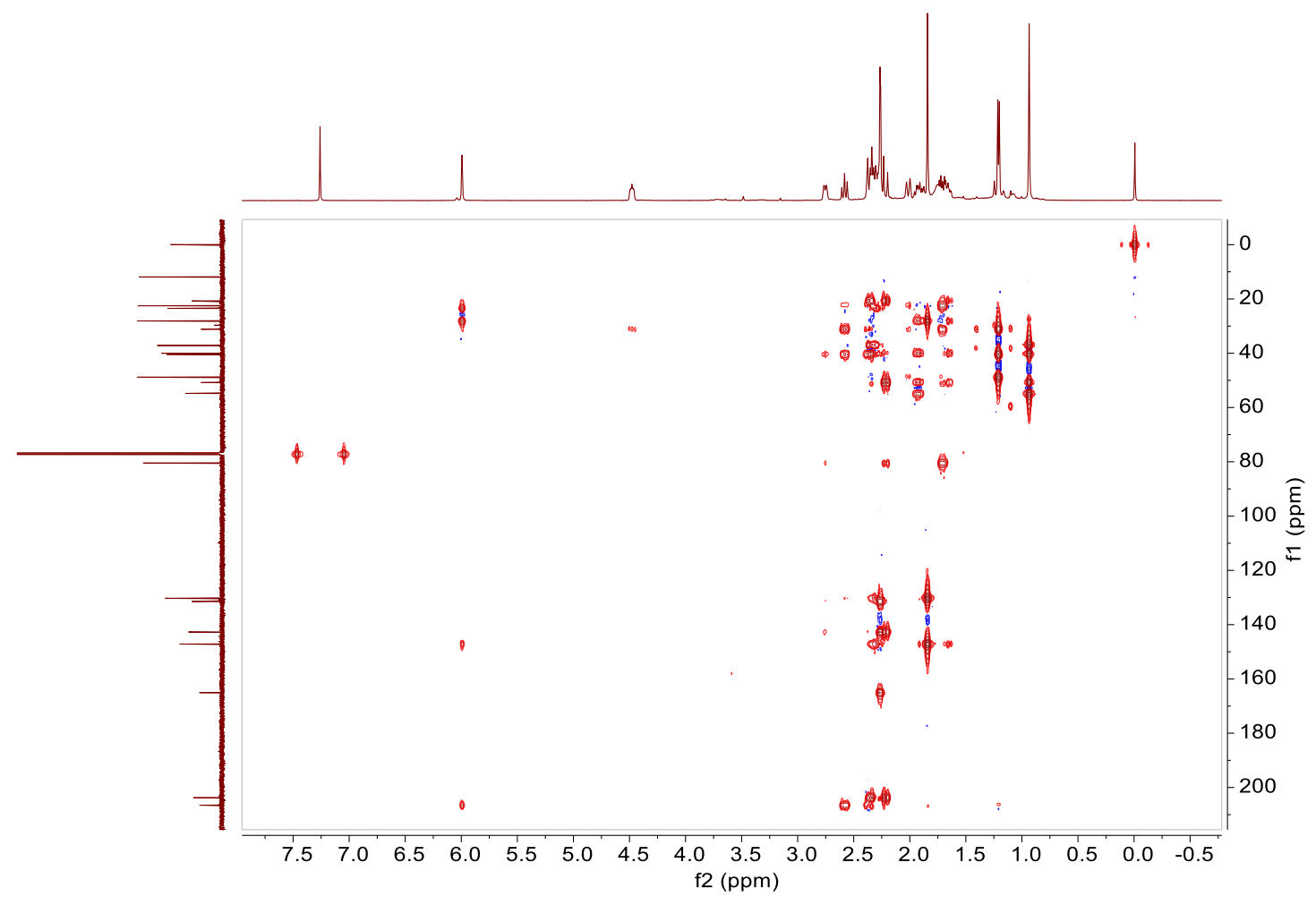

Figure S15. HMBC spectrum of compound 1 in $\mathrm{CDCl}_{3}$.

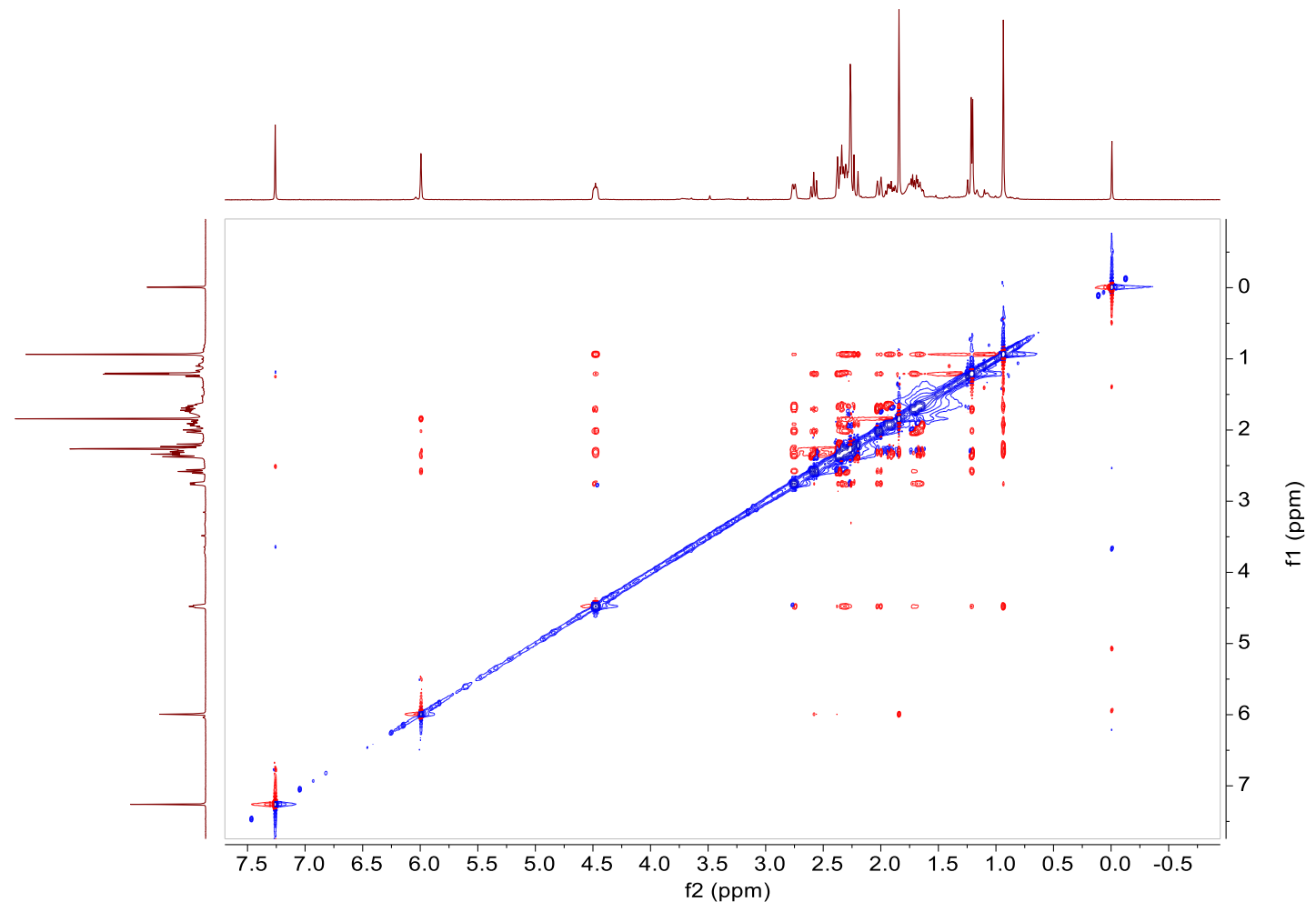

Figure S16. NOESY spectrum of compound 1 in $\mathrm{CDCl}_{3}$. 


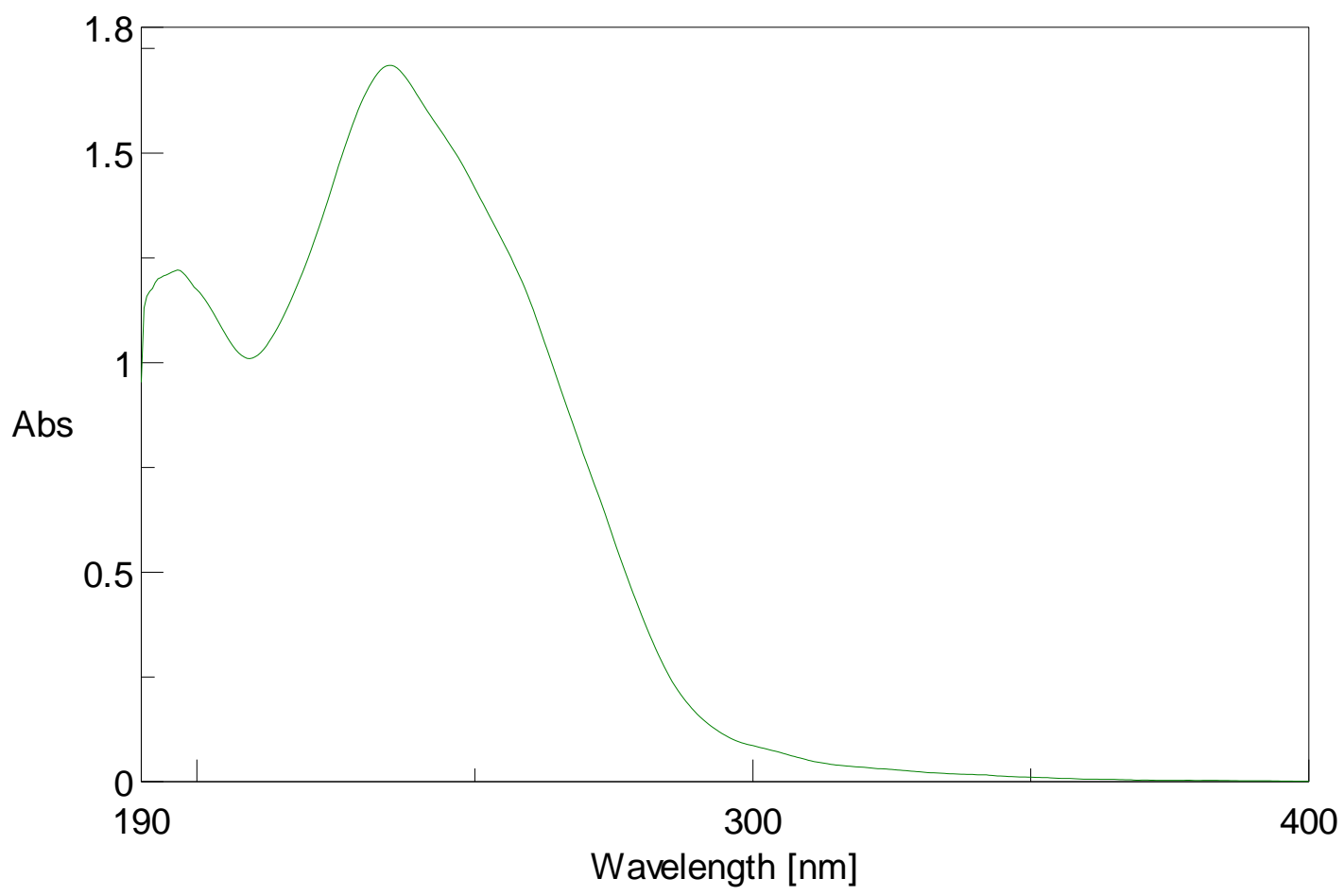

Figure S17. UV spectrum of compound 1.

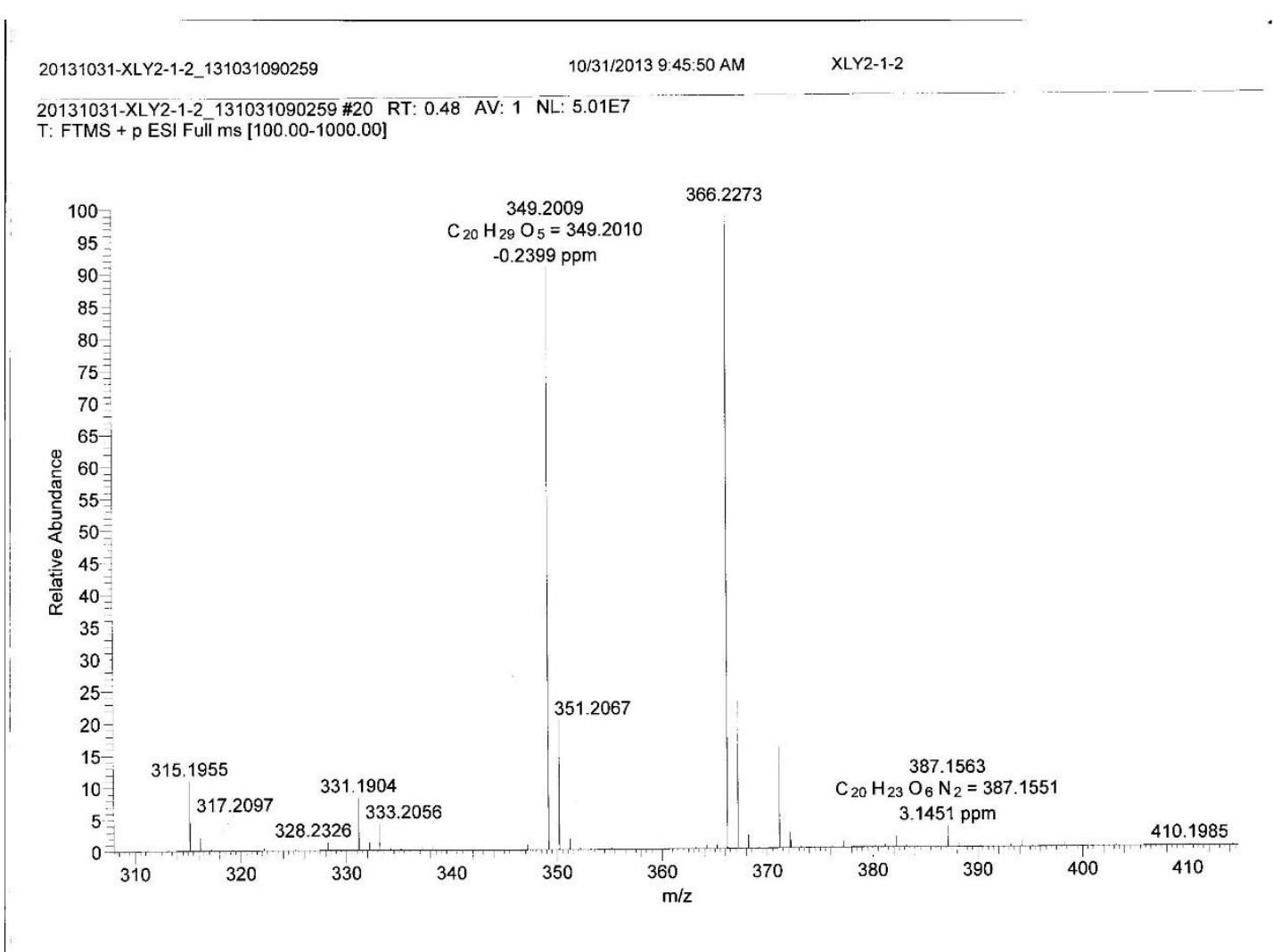

Figure S18. HRESIMS spectrum of compound 2. 


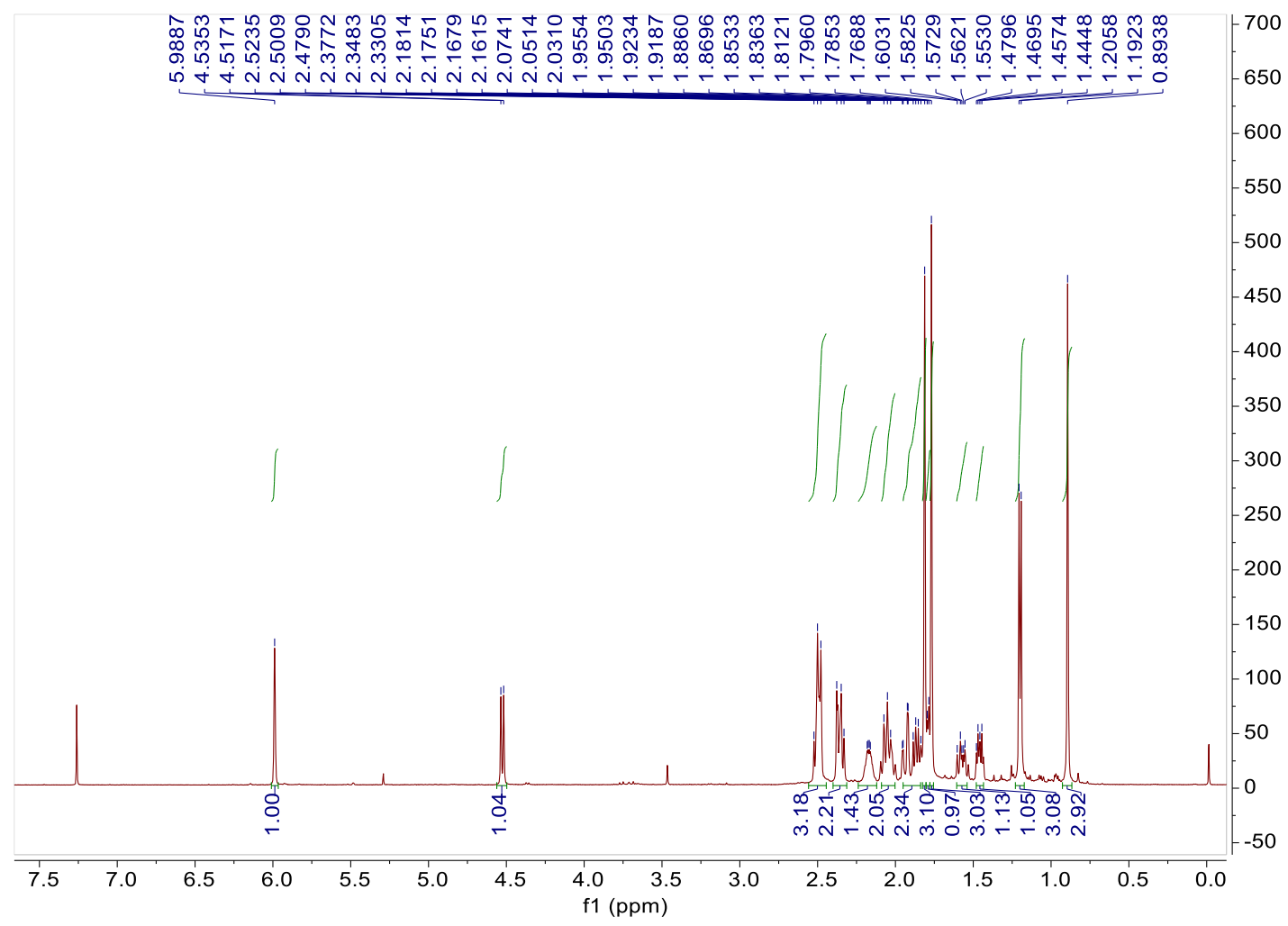

Figure S19. ${ }^{1} \mathrm{H}$ NMR spectrum of compound 2 in $\mathrm{CDCl}_{3}$.

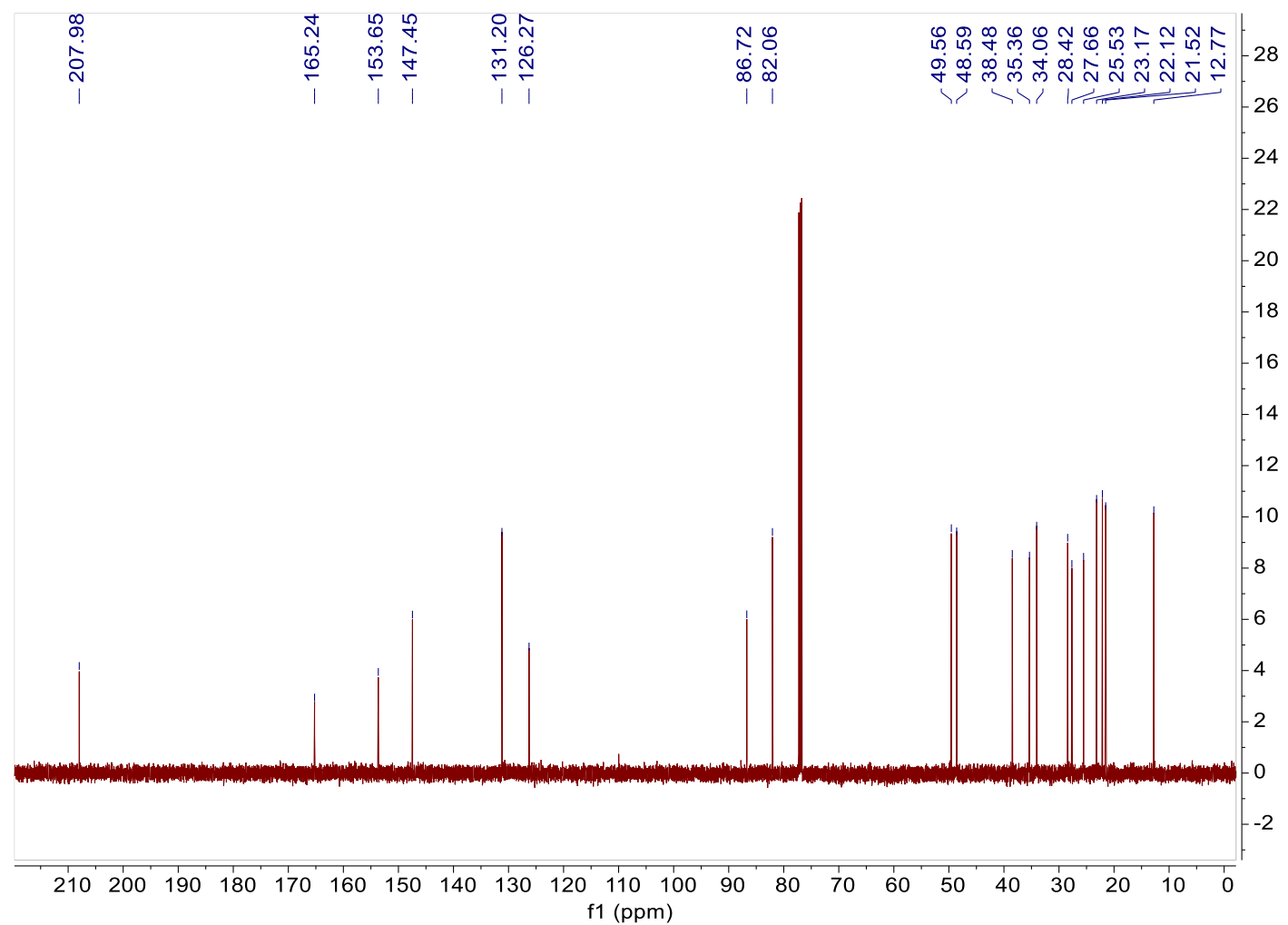

Figure S20. ${ }^{13} \mathrm{C}$ NMR spectrum of compound $\mathbf{2}$ in $\mathrm{CDCl}_{3}$. 


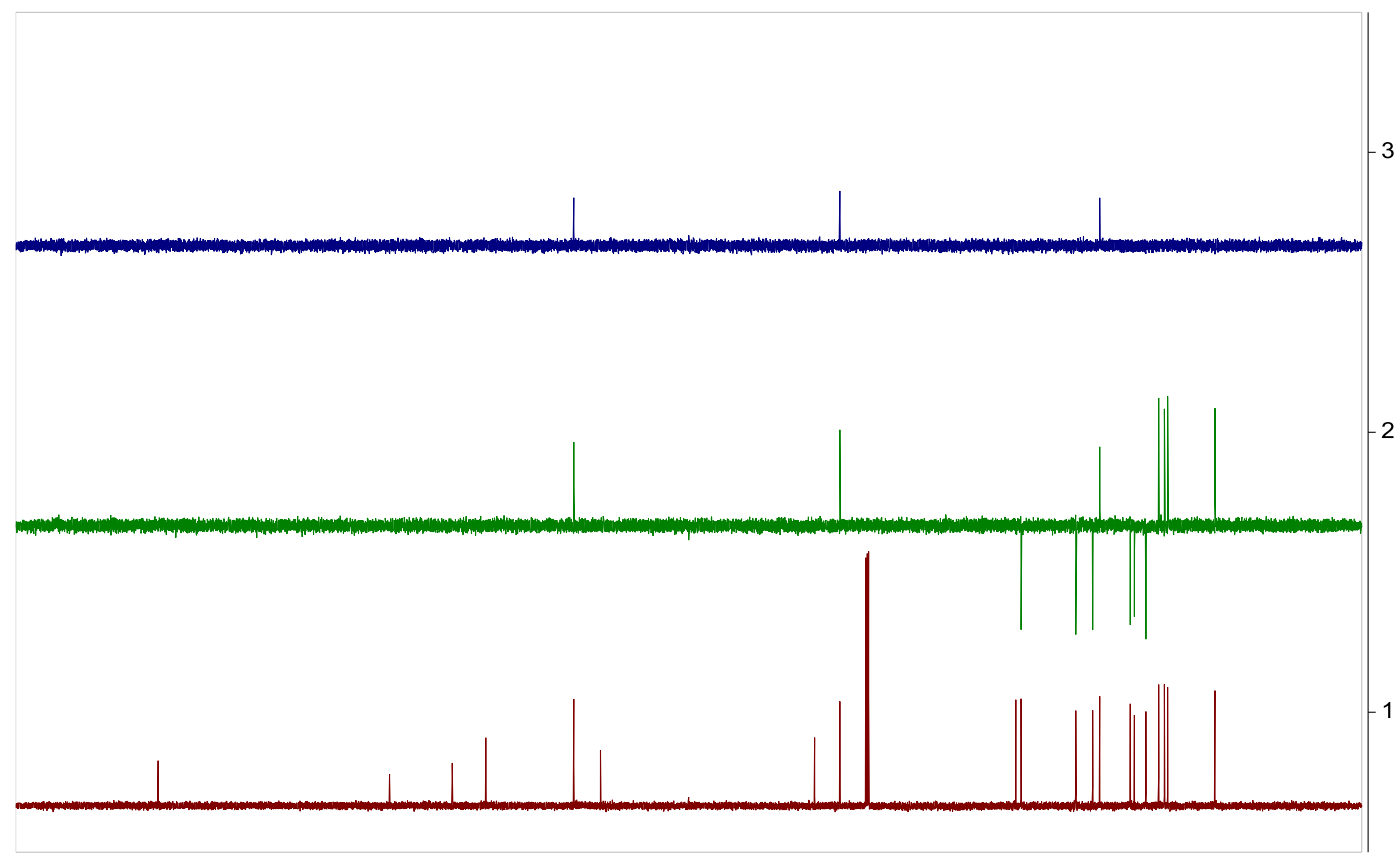

$230 \begin{array}{lllllllllllllllllllllllllll} & 220 & 210 & 200 & 190 & 180 & 170 & 160 & 150 & 140 & 130 & 120 & 110 & 100 & 90 & 80 & 70 & 60 & 50 & 40 & 30 & 20 & 10 & 0 & -10\end{array}$ f1 (ppm)

Figure S21. ${ }^{13} \mathrm{C}$ and DEPT spectrum of compound 2 in $\mathrm{CDCl}_{3}$.

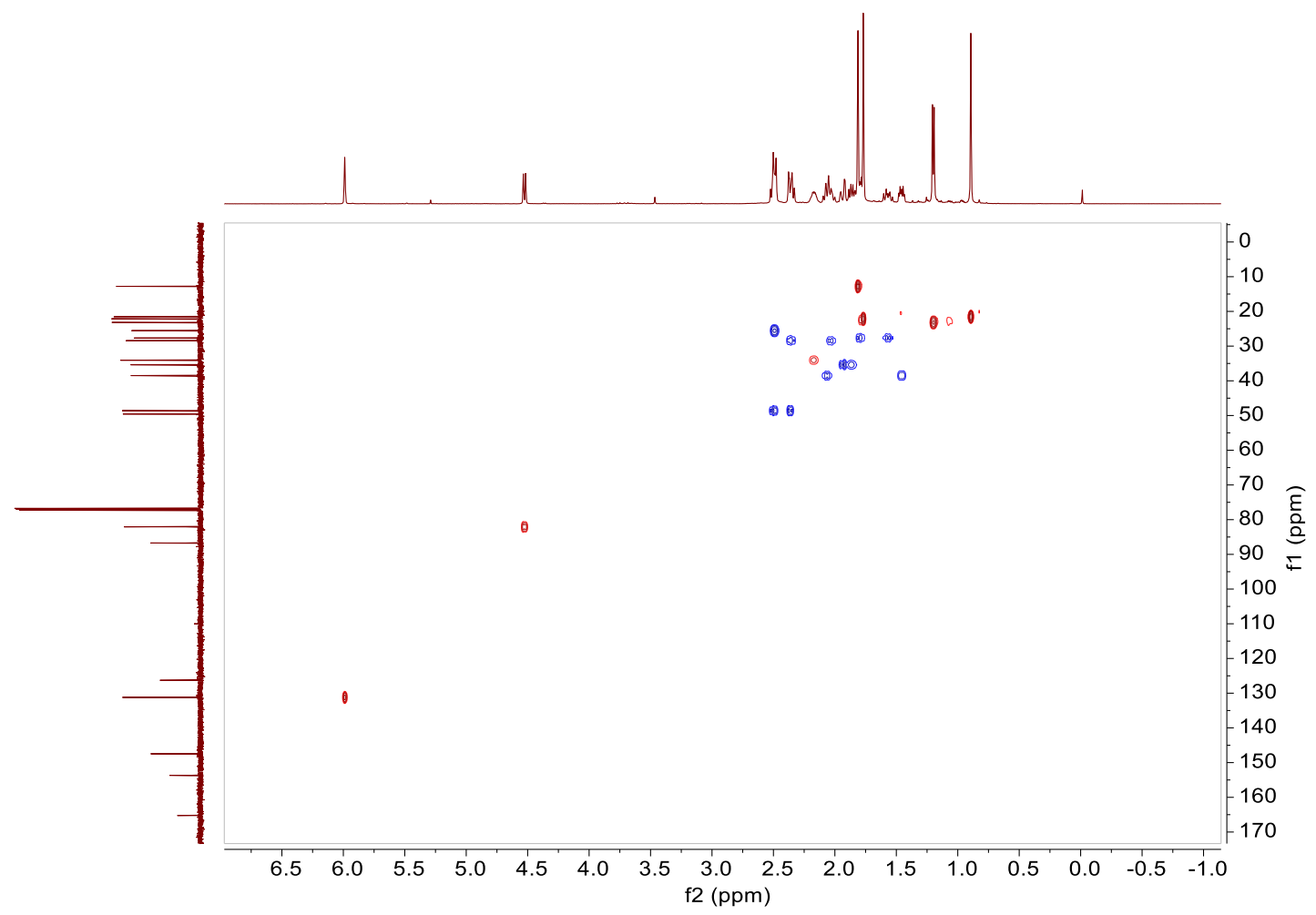

Figure S22. HSQC spectrum of compound 2 in $\mathrm{CDCl}_{3}$. 


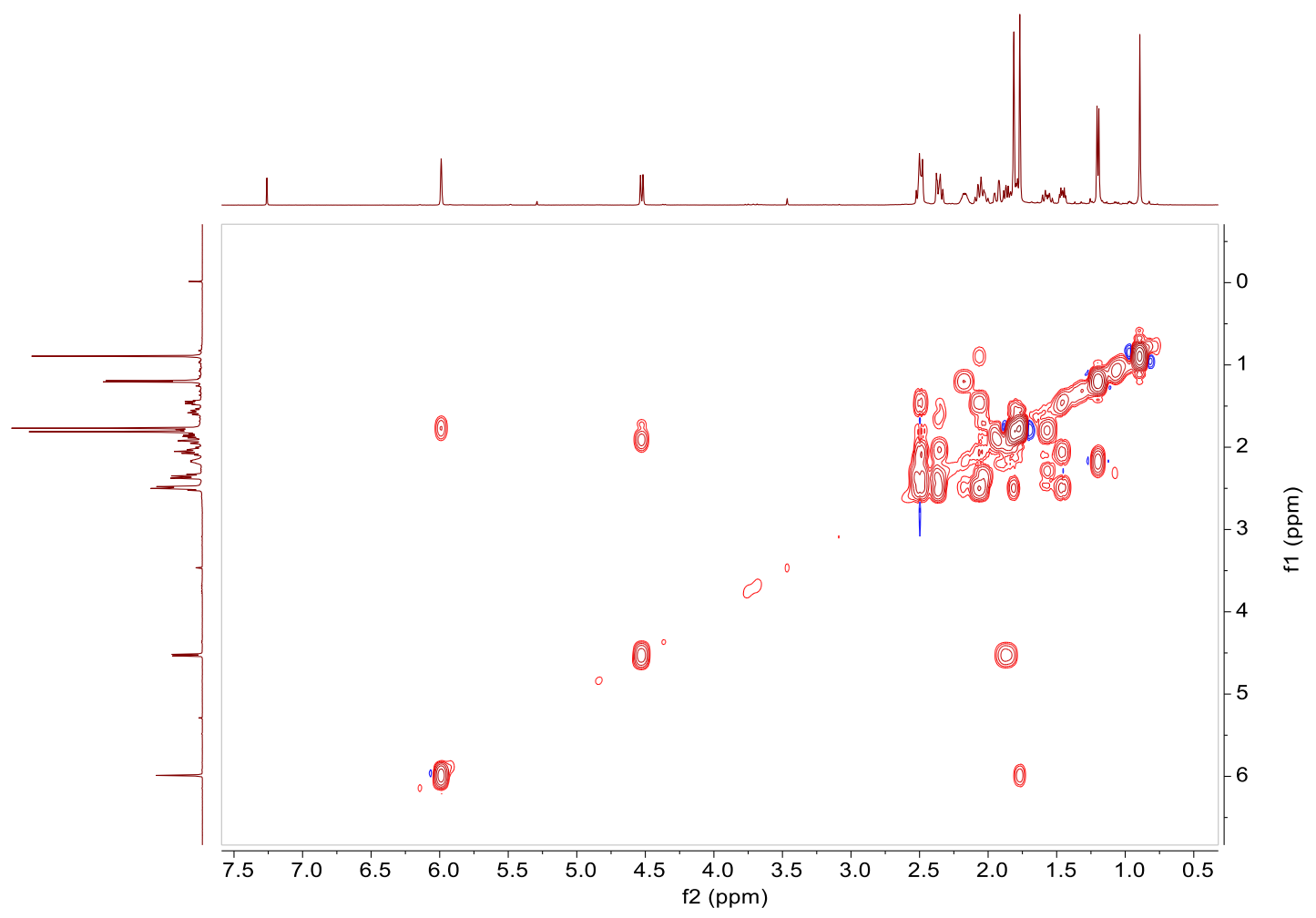

Figure S23. ${ }^{1} \mathrm{H}-{ }^{1} \mathrm{H}-\mathrm{COSY}$ spectrum of compound $\mathbf{2}$ in $\mathrm{CDCl}_{3}$.

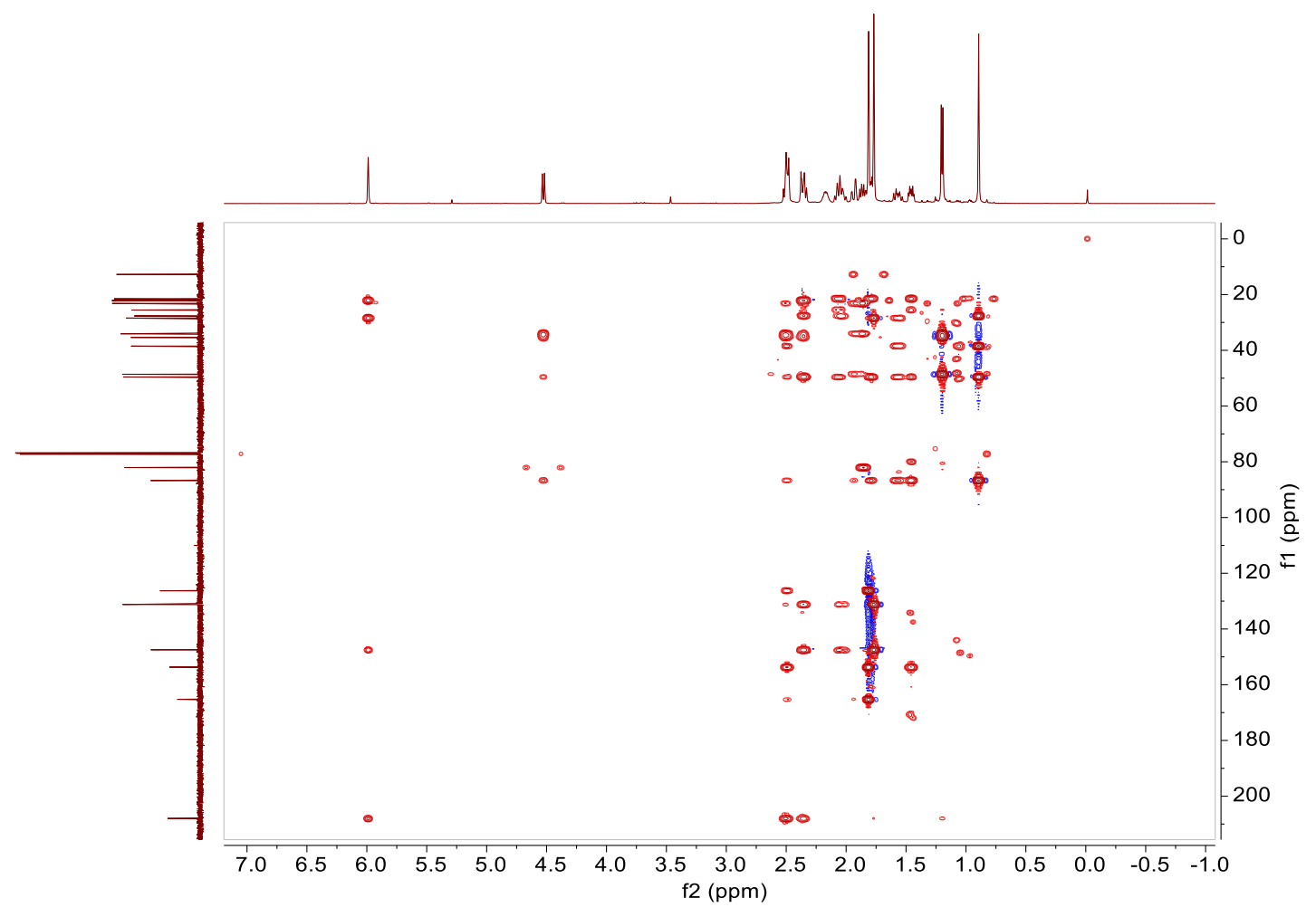

Figure S24. HMBC spectrum of compound 2 in $\mathrm{CDCl}_{3}$. 


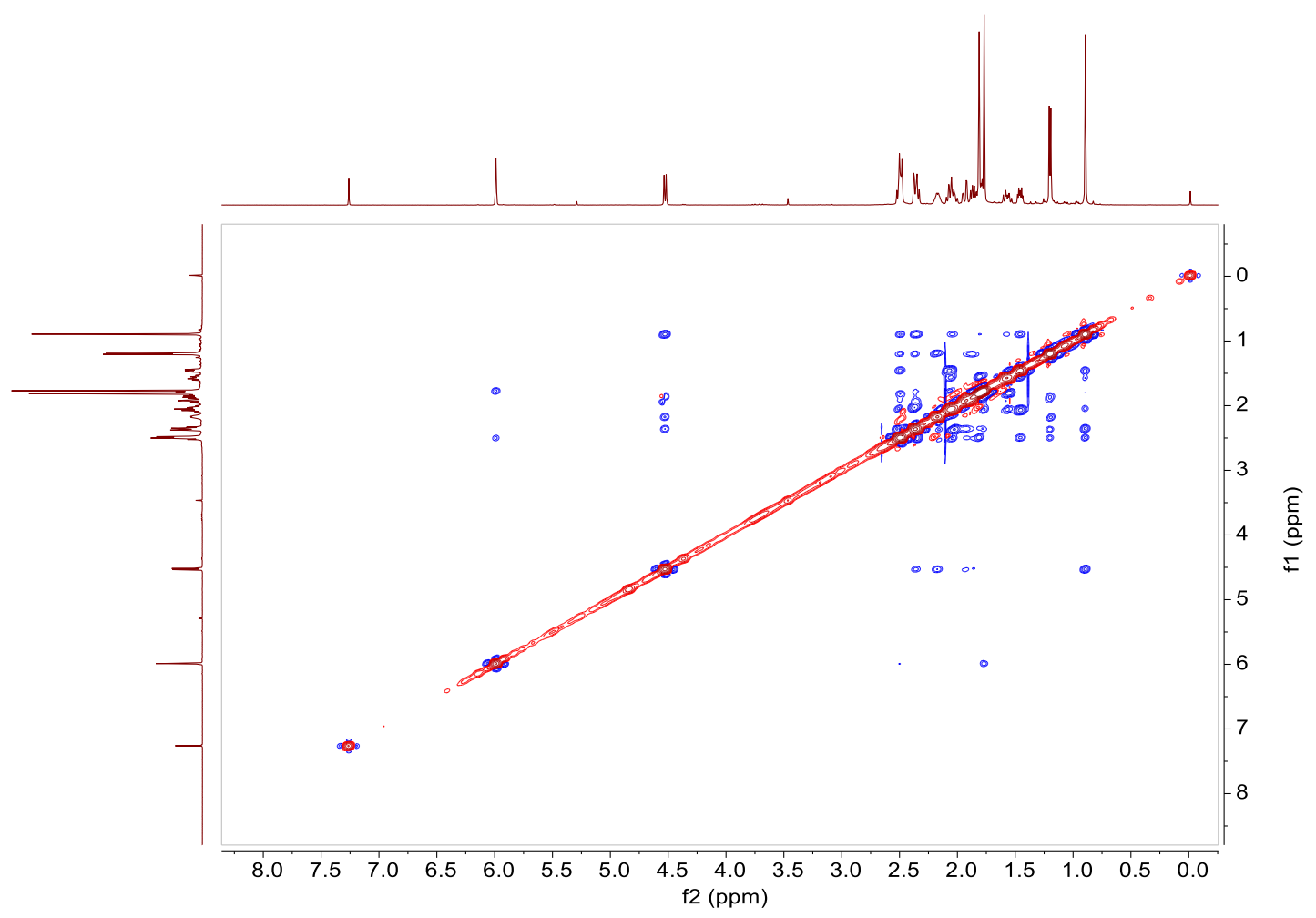

Figure S25. NOESY spectrum of compound 2 in $\mathrm{CDCl}_{3}$.

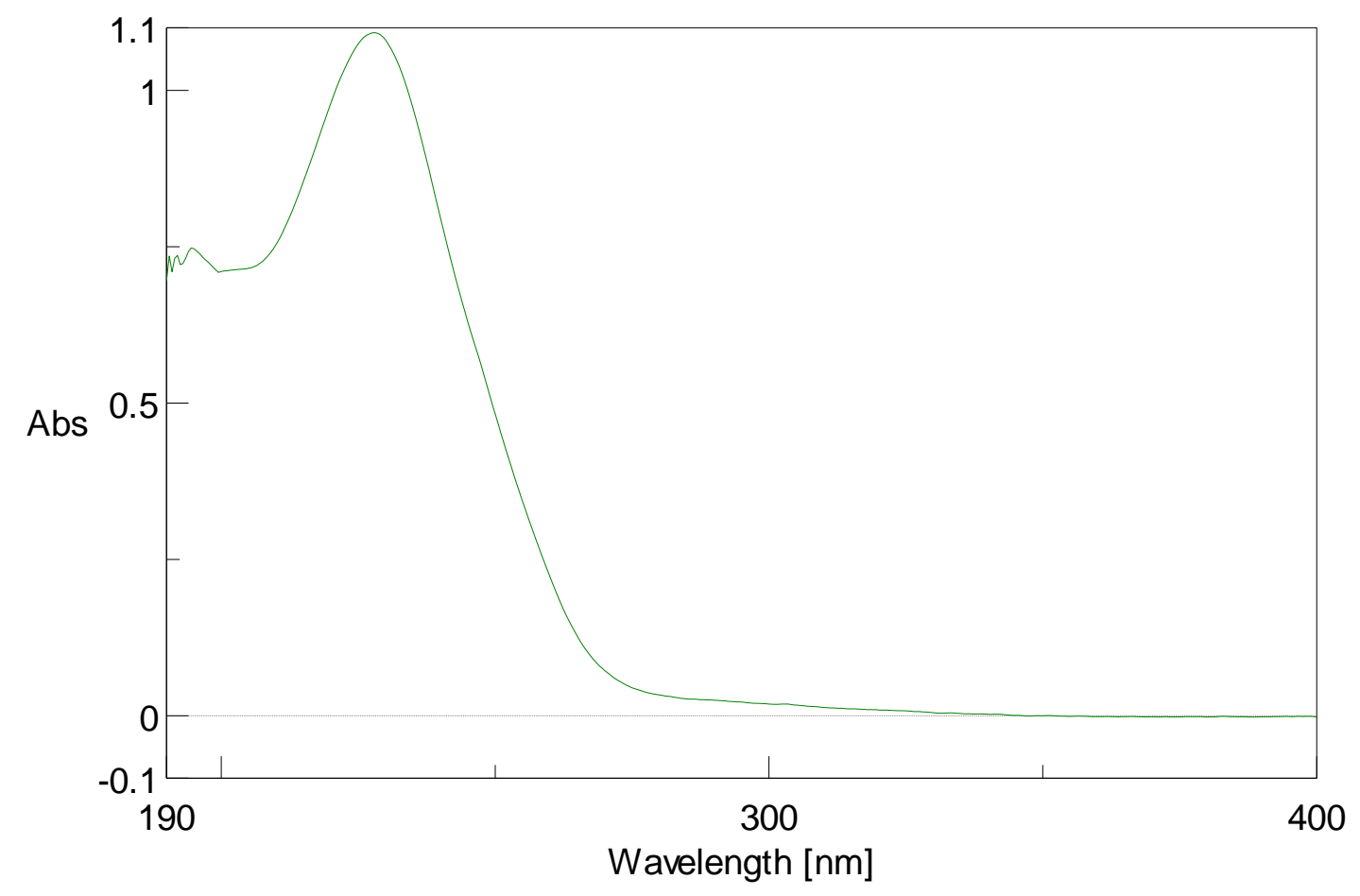

Figure S26. UV spectrum of compound 2 


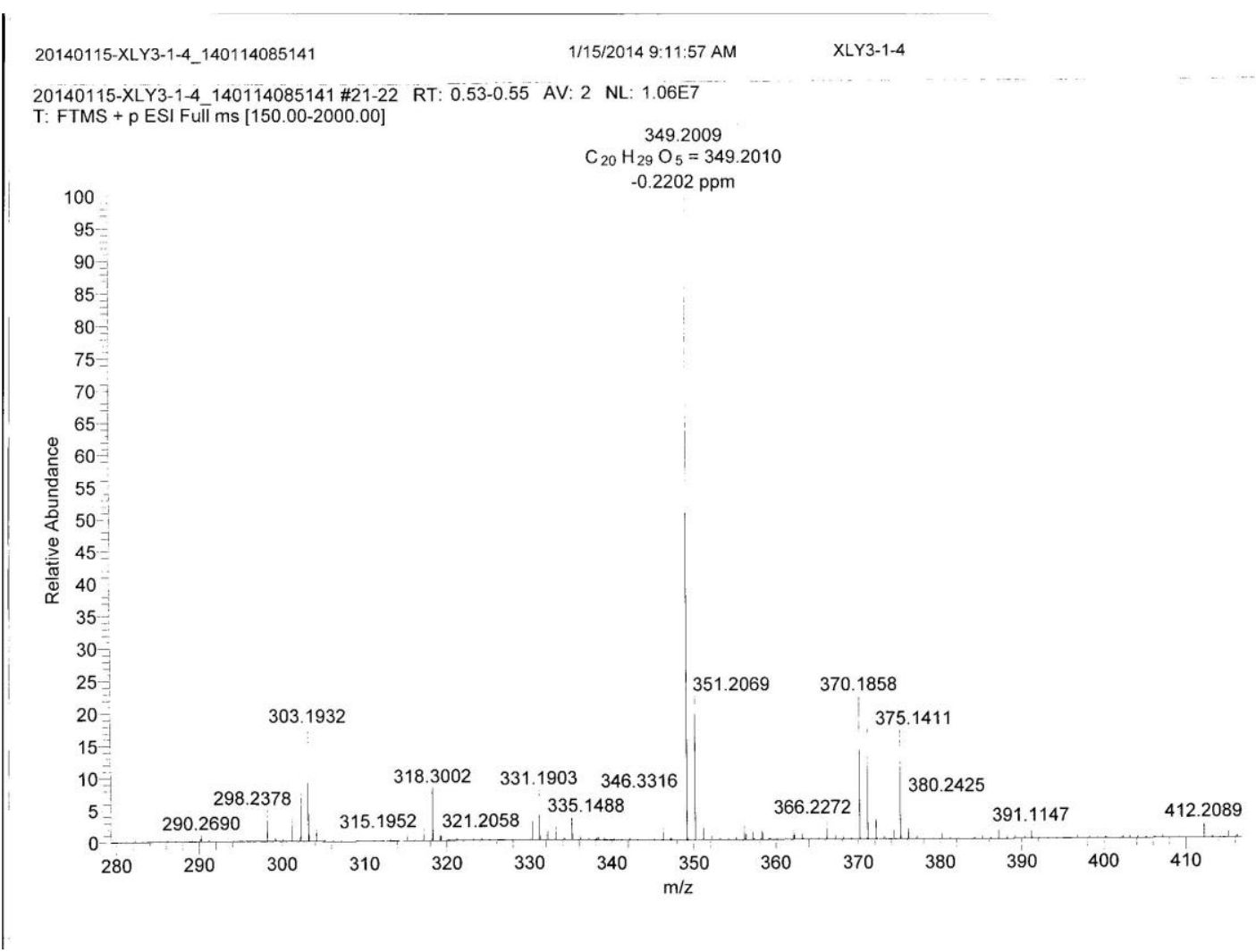

Figure S27. HRESIMS spectrum of compound $\mathbf{3}$

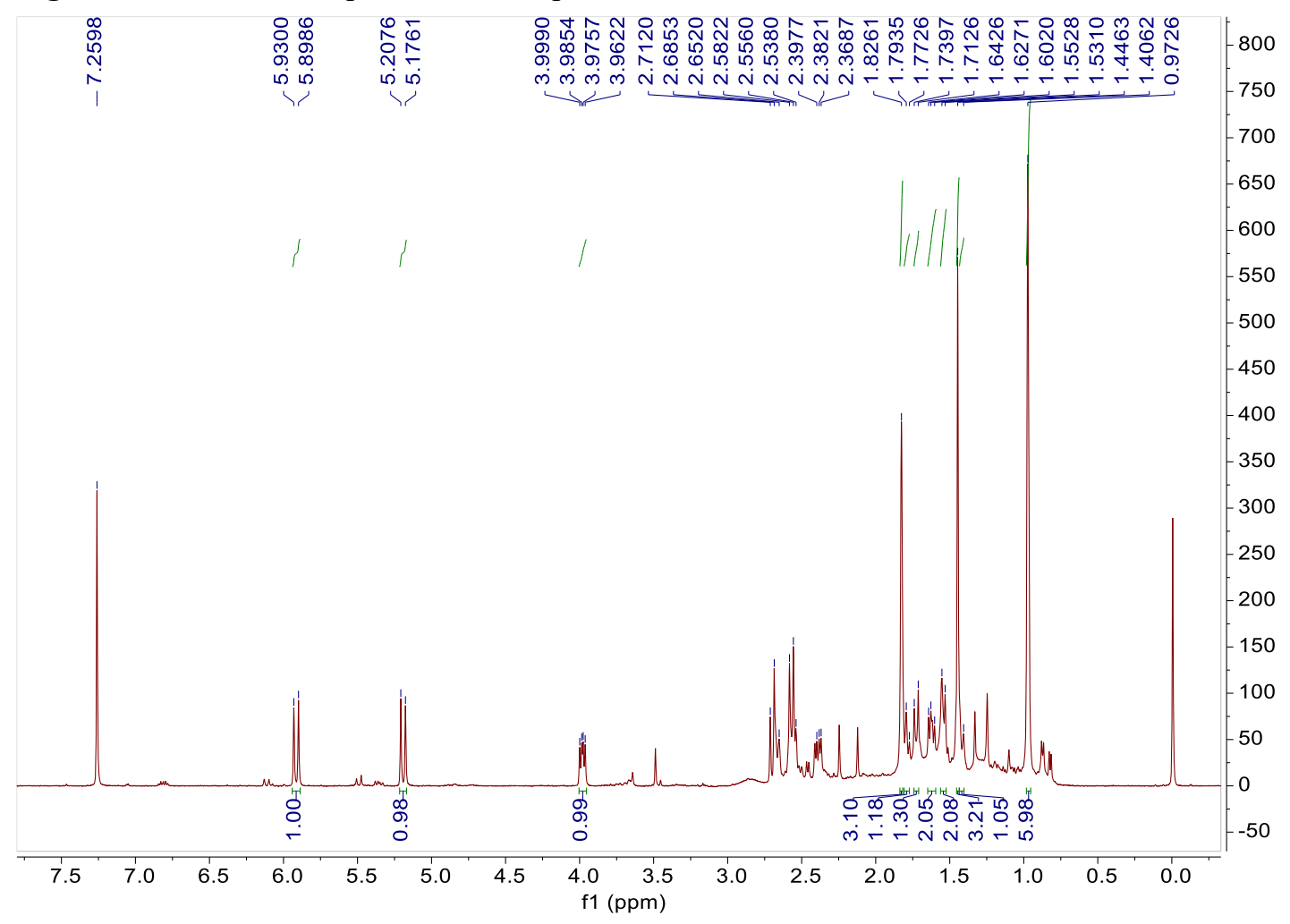

Figure S28. ${ }^{1} \mathrm{H}$ NMR spectrum of compound 3 in $\mathrm{CDCl}_{3}$. 


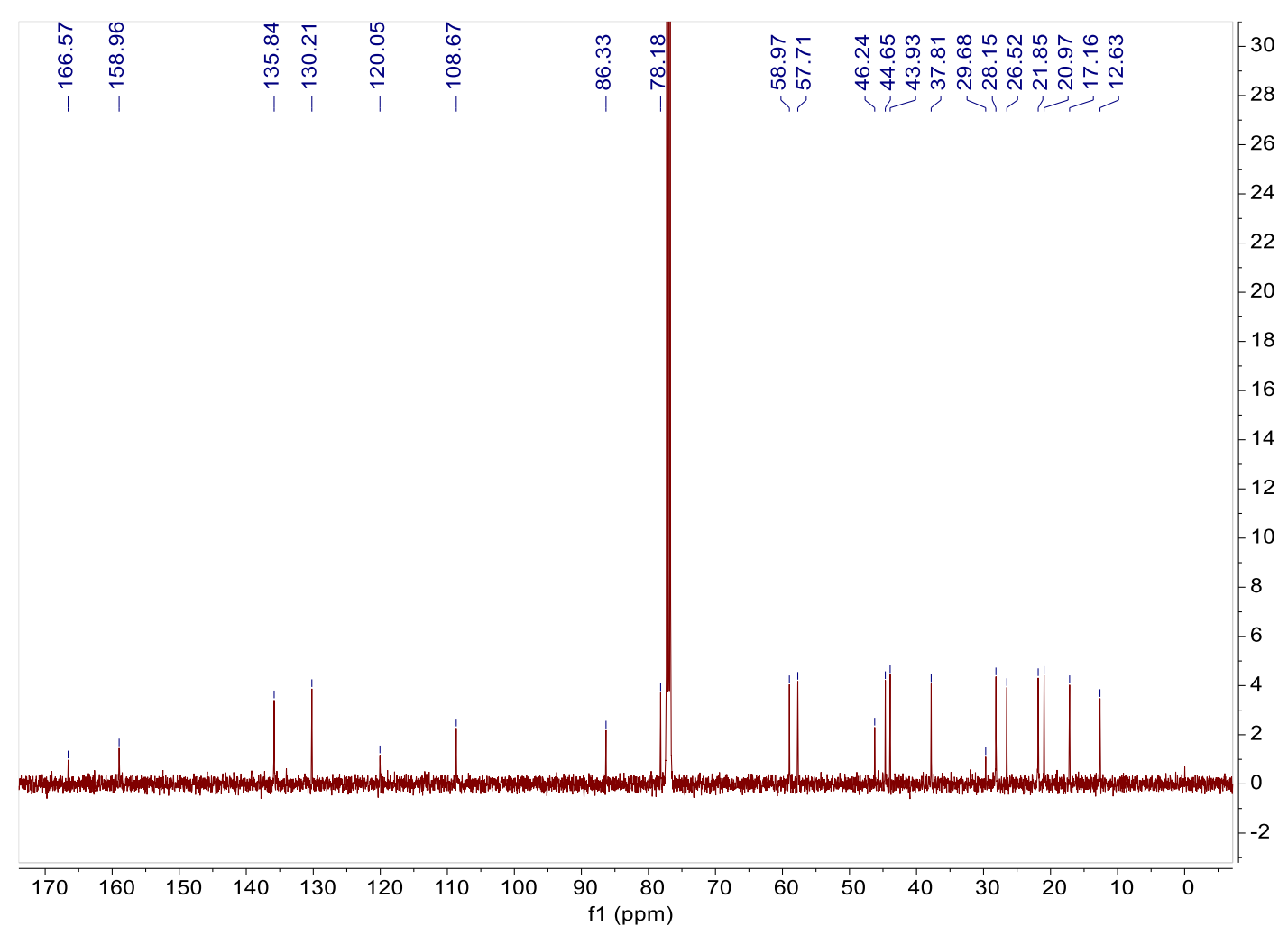

Figure S29. ${ }^{13} \mathrm{C}$ NMR spectrum of compound 3 in $\mathrm{CDCl}_{3}$.

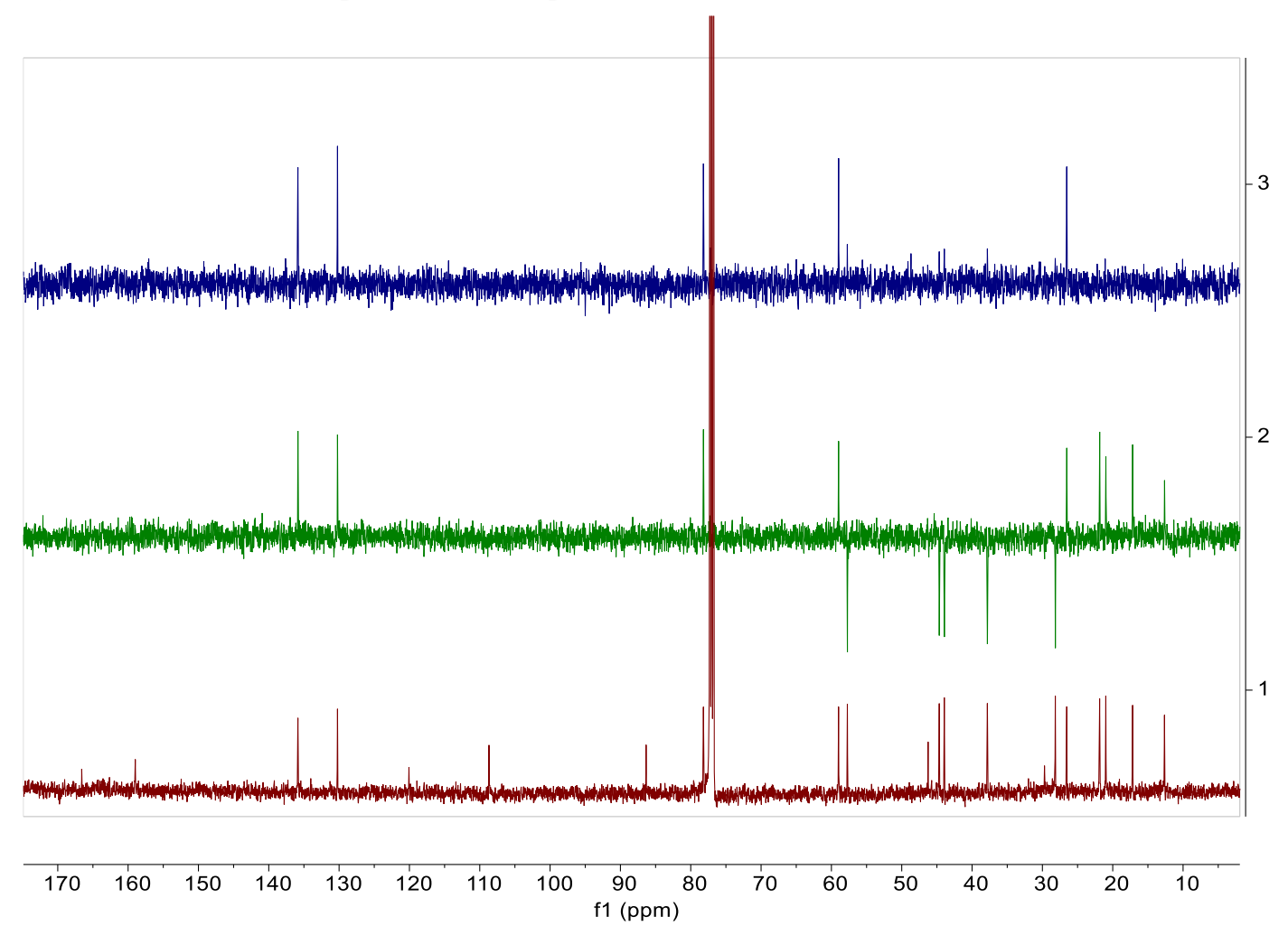

Figure S30. ${ }^{13} \mathrm{C}$ and DEPT spectrum of compound 3 in $\mathrm{CDCl}_{3}$. 


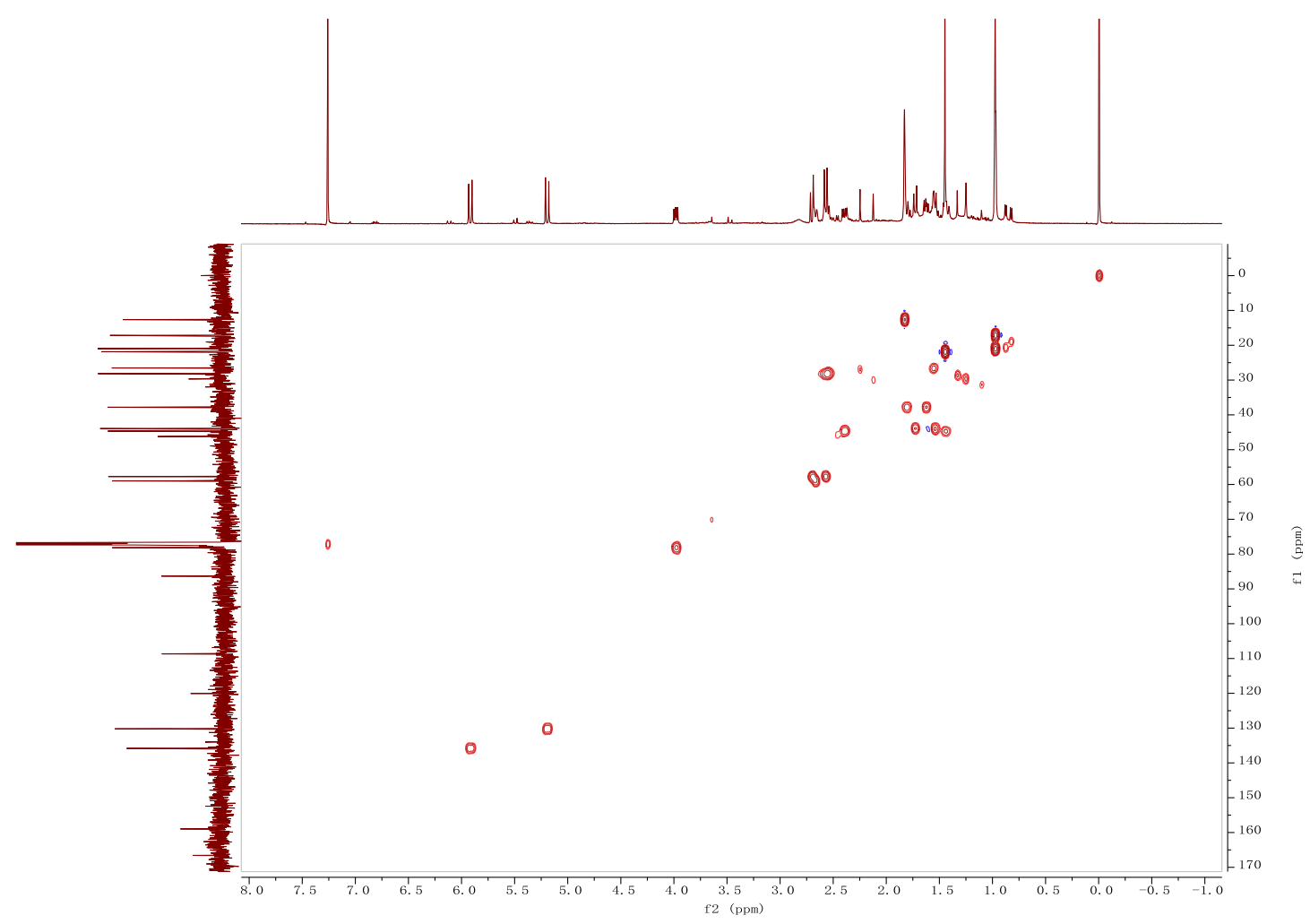

Figure S31. HSQC spectrum of compound 3 in $\mathrm{CDCl}_{3}$.

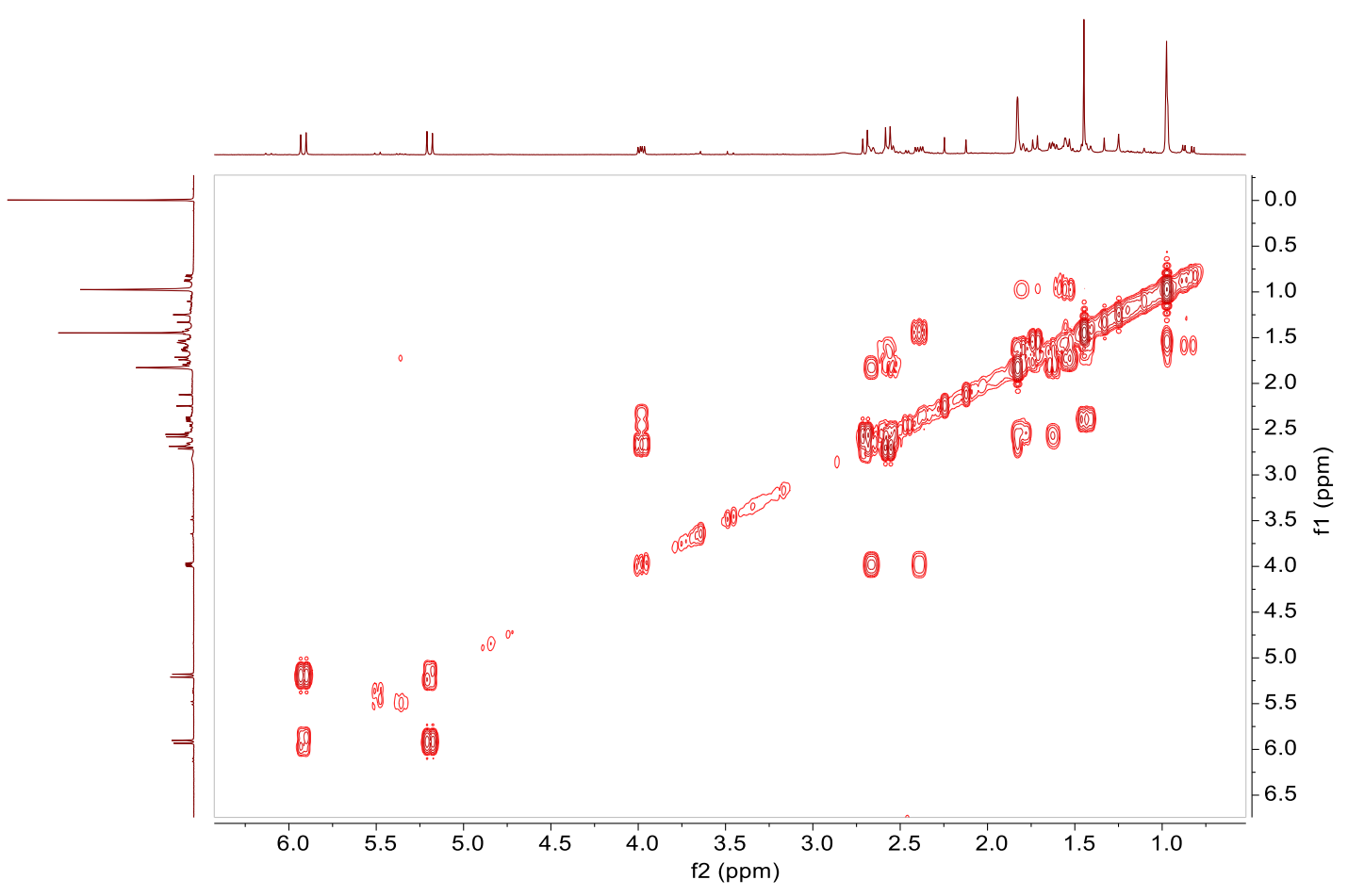

Figure S32. ${ }^{1} \mathrm{H}-{ }^{1} \mathrm{H}-\mathrm{COSY}$ spectrum of compound 3 in $\mathrm{CDCl}_{3}$. 


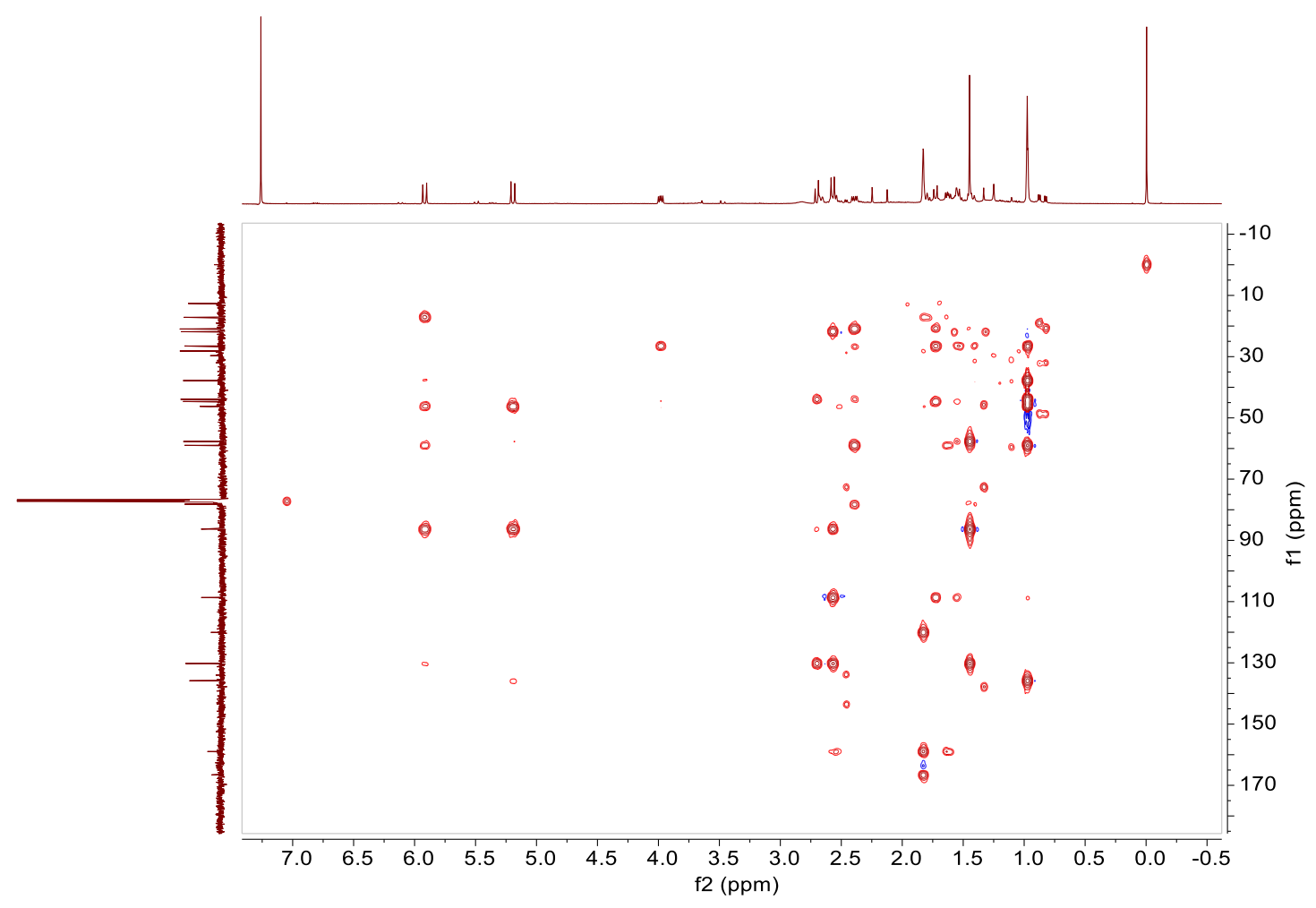

Figure S33. HMBC spectrum of compound $\mathbf{3}$ in $\mathrm{CDCl}_{3}$.

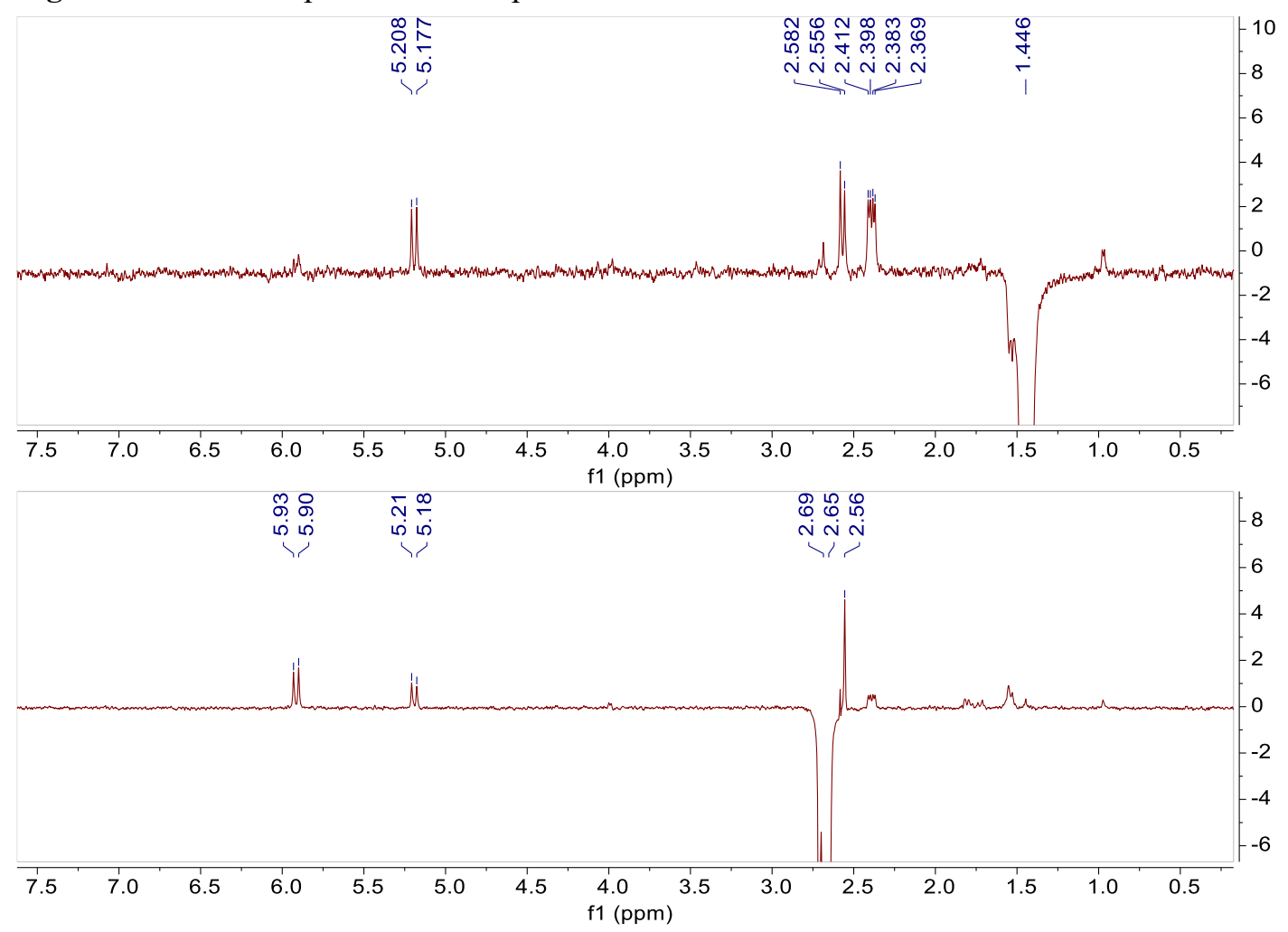

Figure S34. 1D NOE spectrum of compound 3 in $\mathrm{CDCl}_{3}$. 


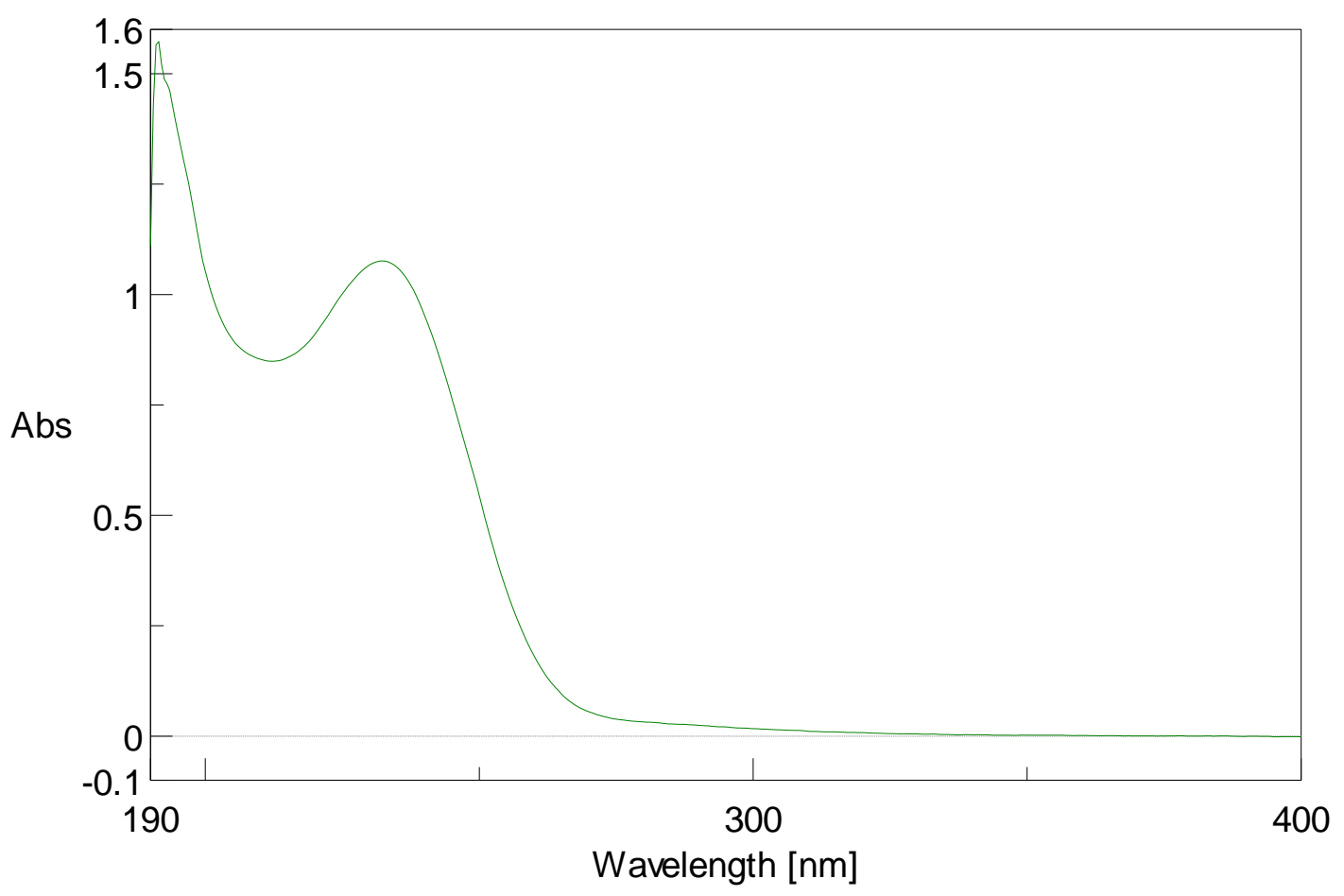

Figure S35. UV spectrum of compound 3.

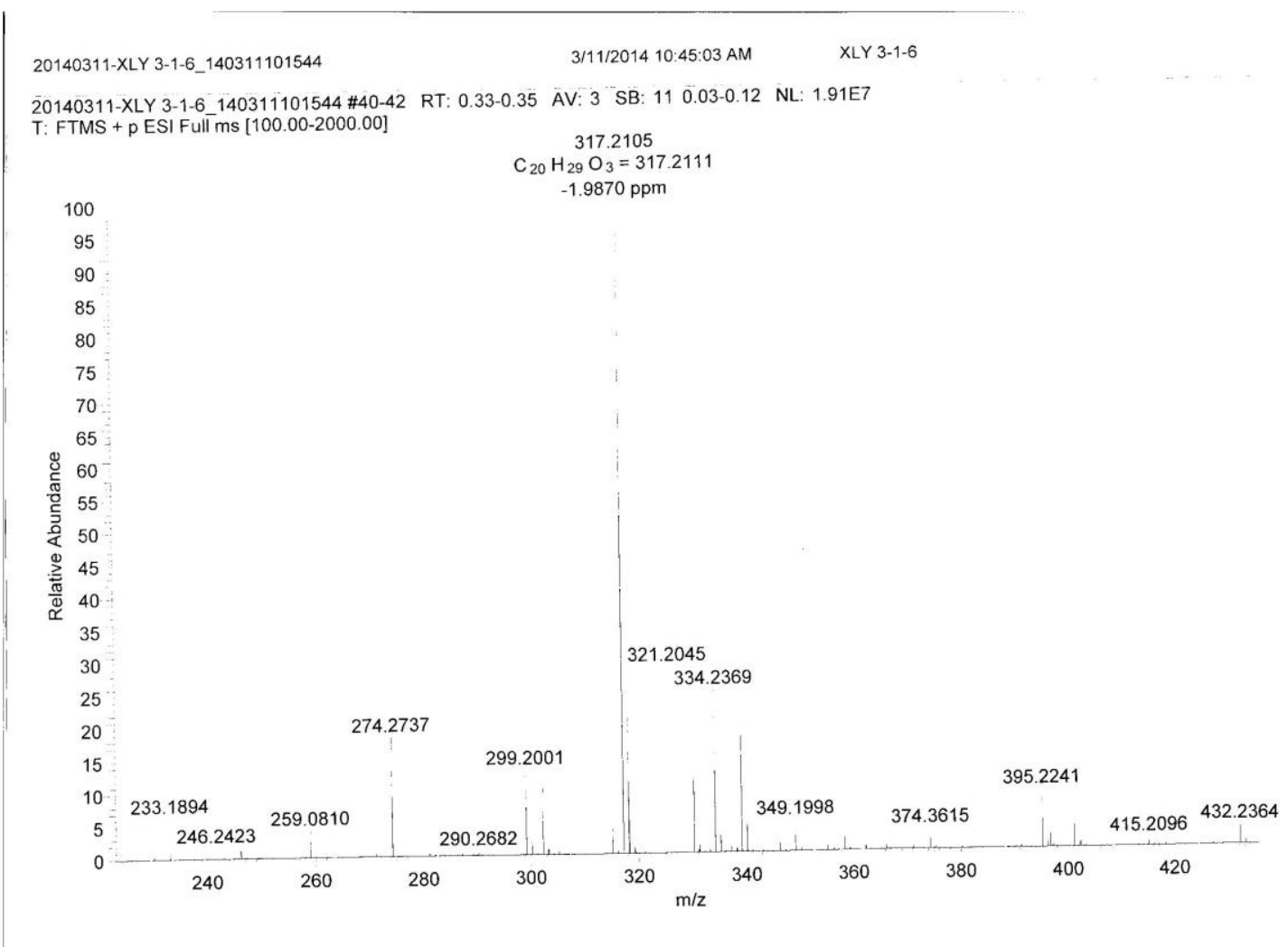

Figure S36. HRESIMS spectrum of compound 4. 


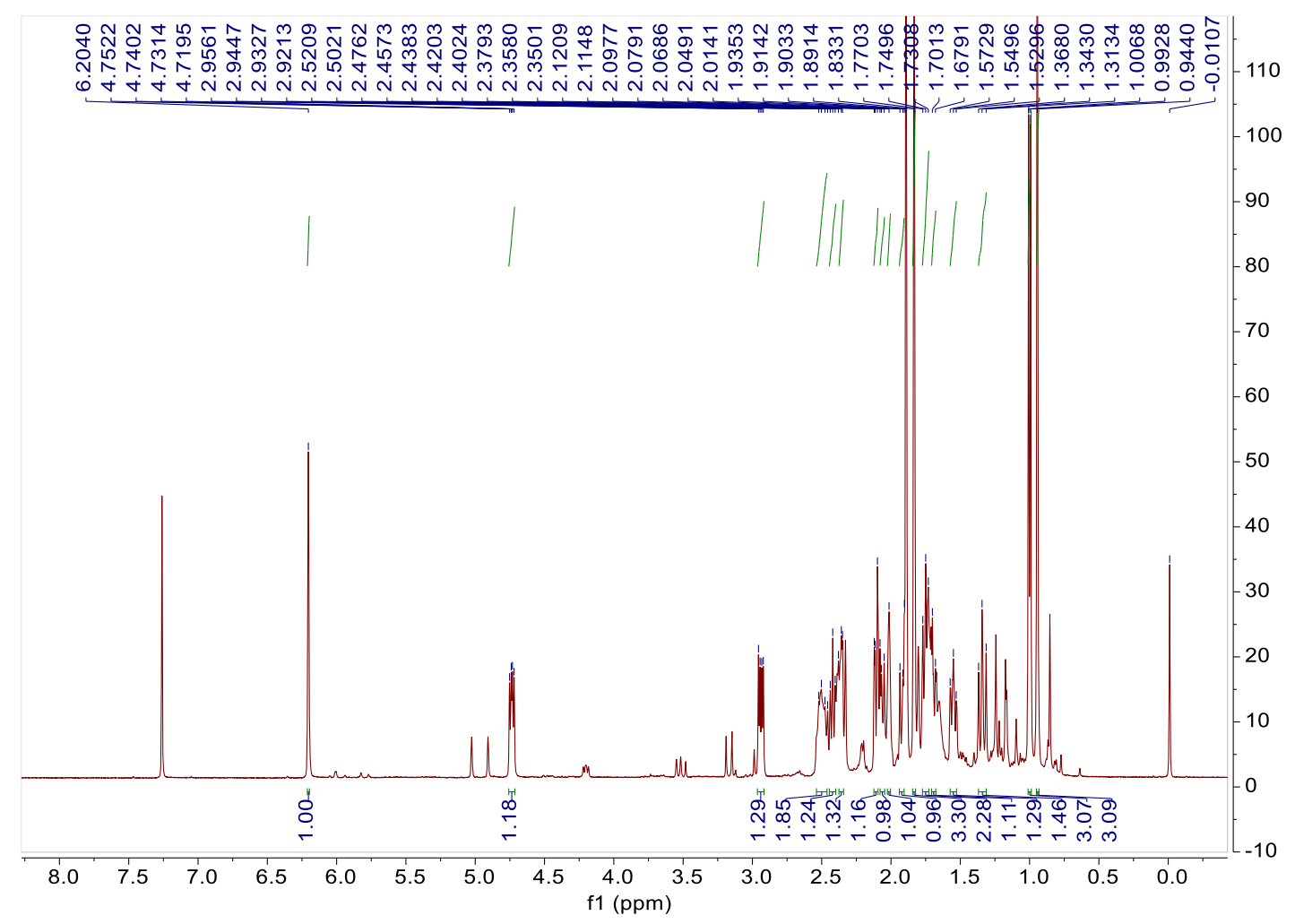

Figure S37. ${ }^{1} \mathrm{H}$ NMR spectrum of compound 4 in $\mathrm{CDCl}_{3}$.

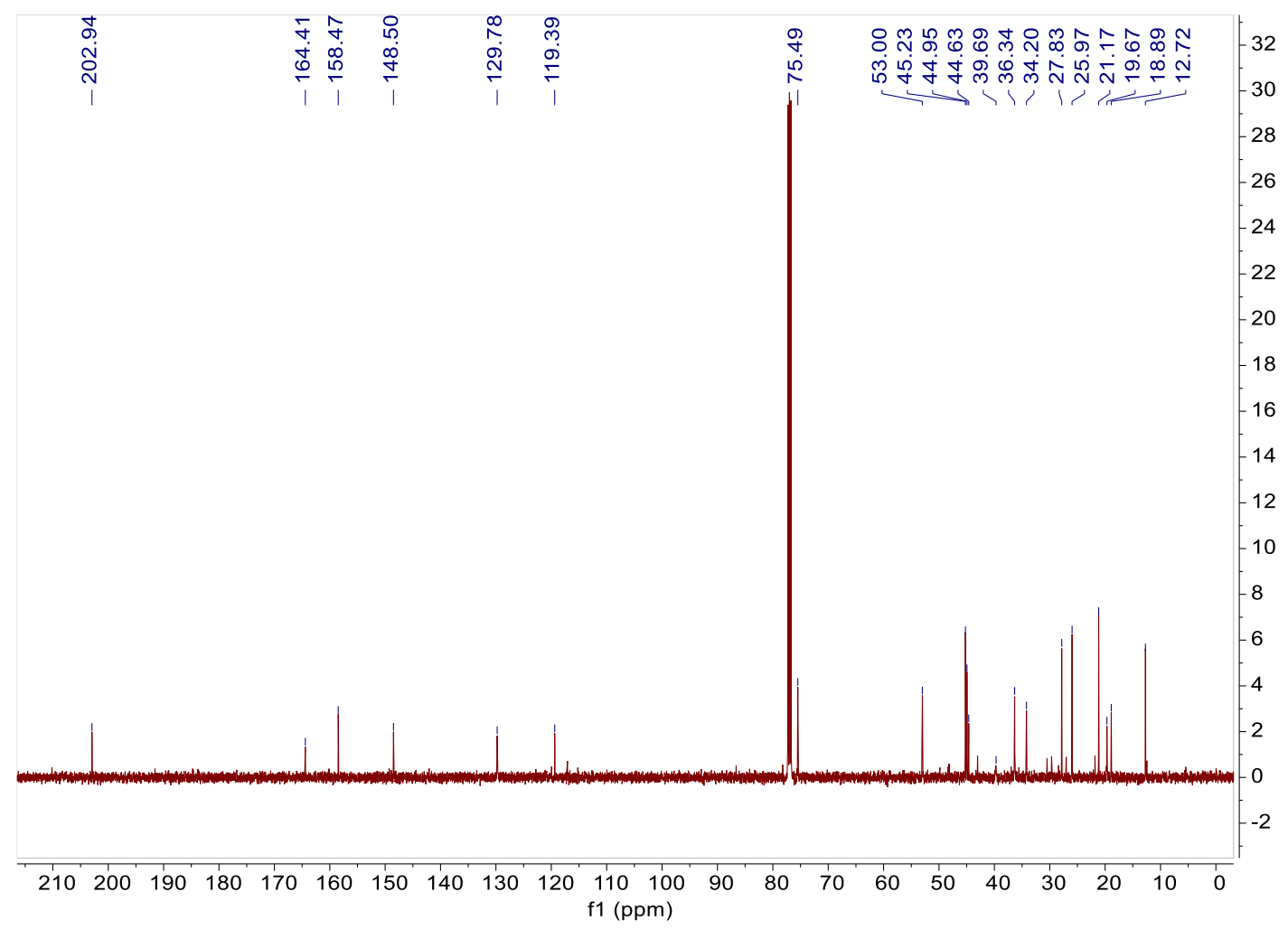

Figure S38. ${ }^{13} \mathrm{C}$ NMR spectrum of compound 4 in $\mathrm{CDCl}_{3}$. 


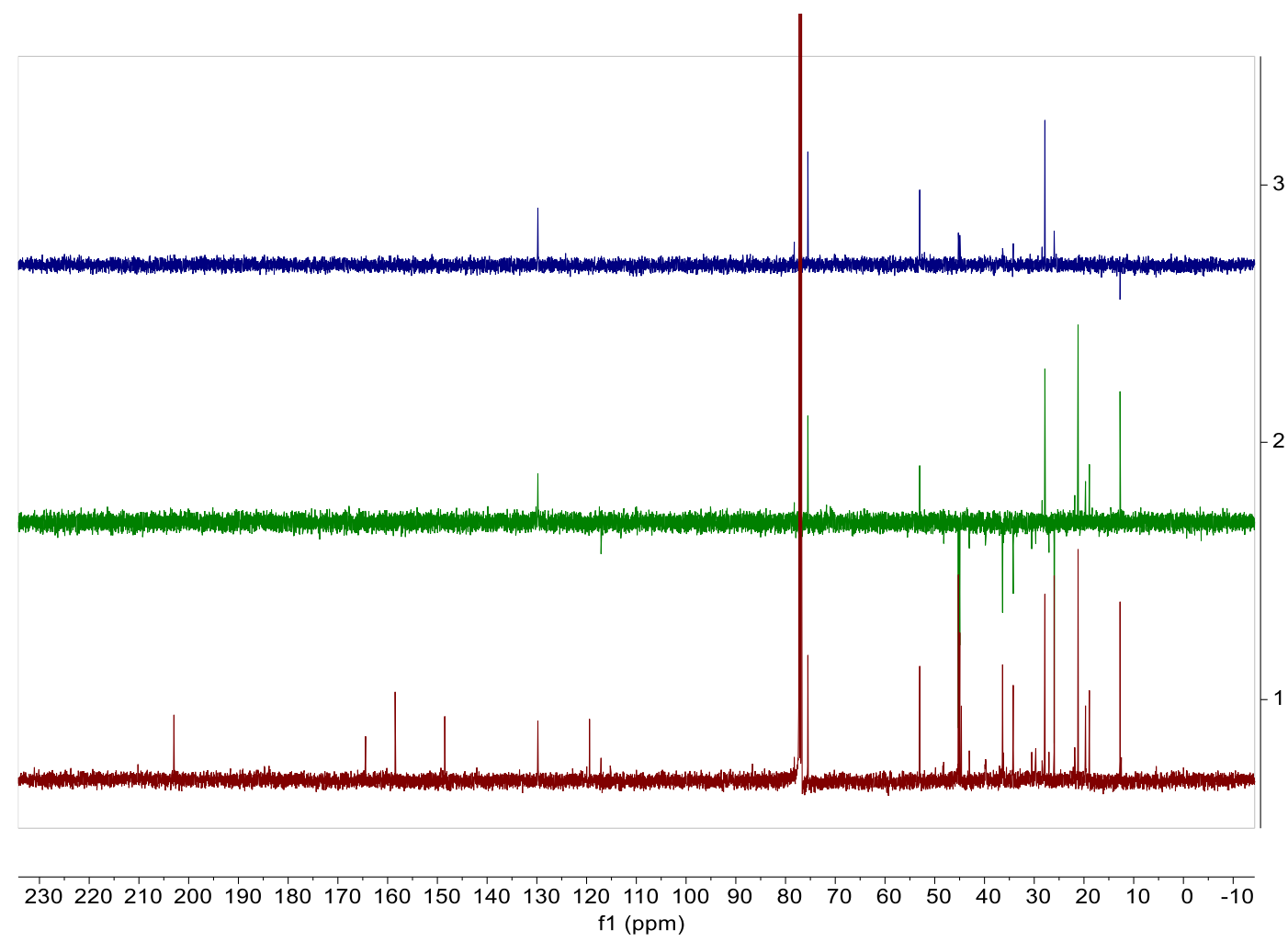

Figure S39. ${ }^{13} \mathrm{C}$ and DEPT spectrum of compound 4 in $\mathrm{CDCl}_{3}$.

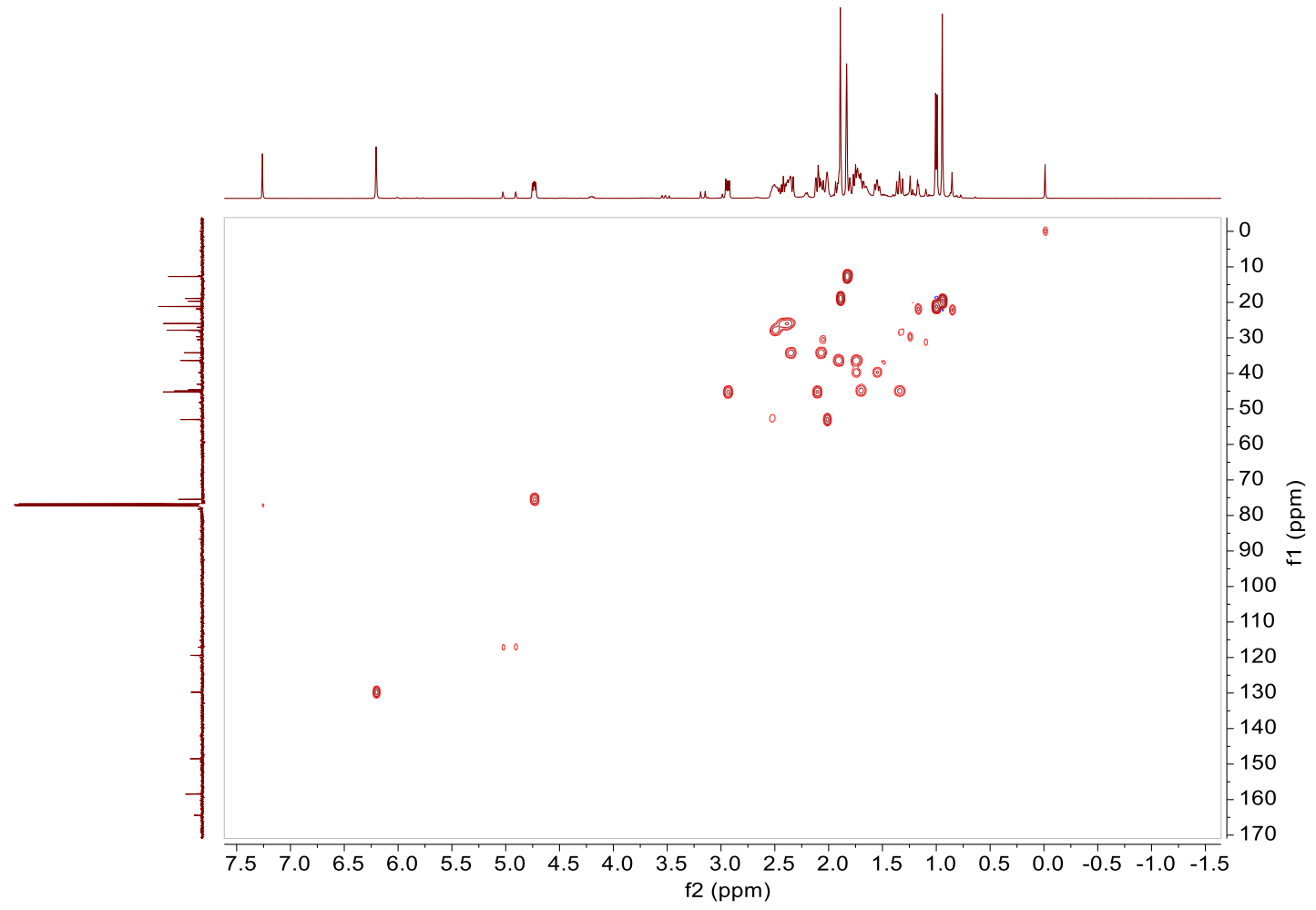

Figure S40. HSQC spectrum of compound 4 in $\mathrm{CDCl}_{3}$. 


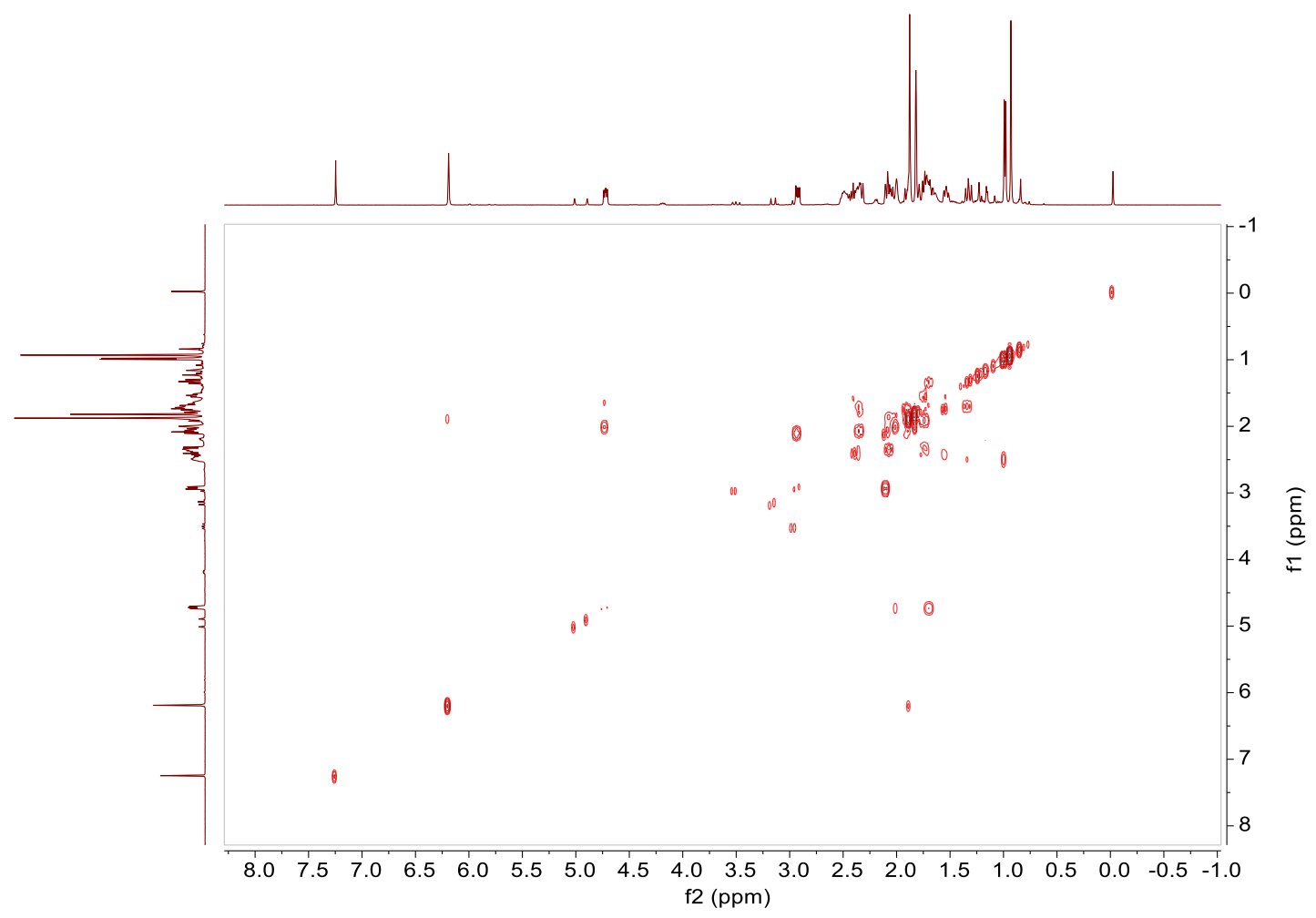

Figure S41. ${ }^{1} \mathrm{H}-{ }^{1} \mathrm{H}-\mathrm{COSY}$ spectrum of compound 4 in $\mathrm{CDCl}_{3}$.

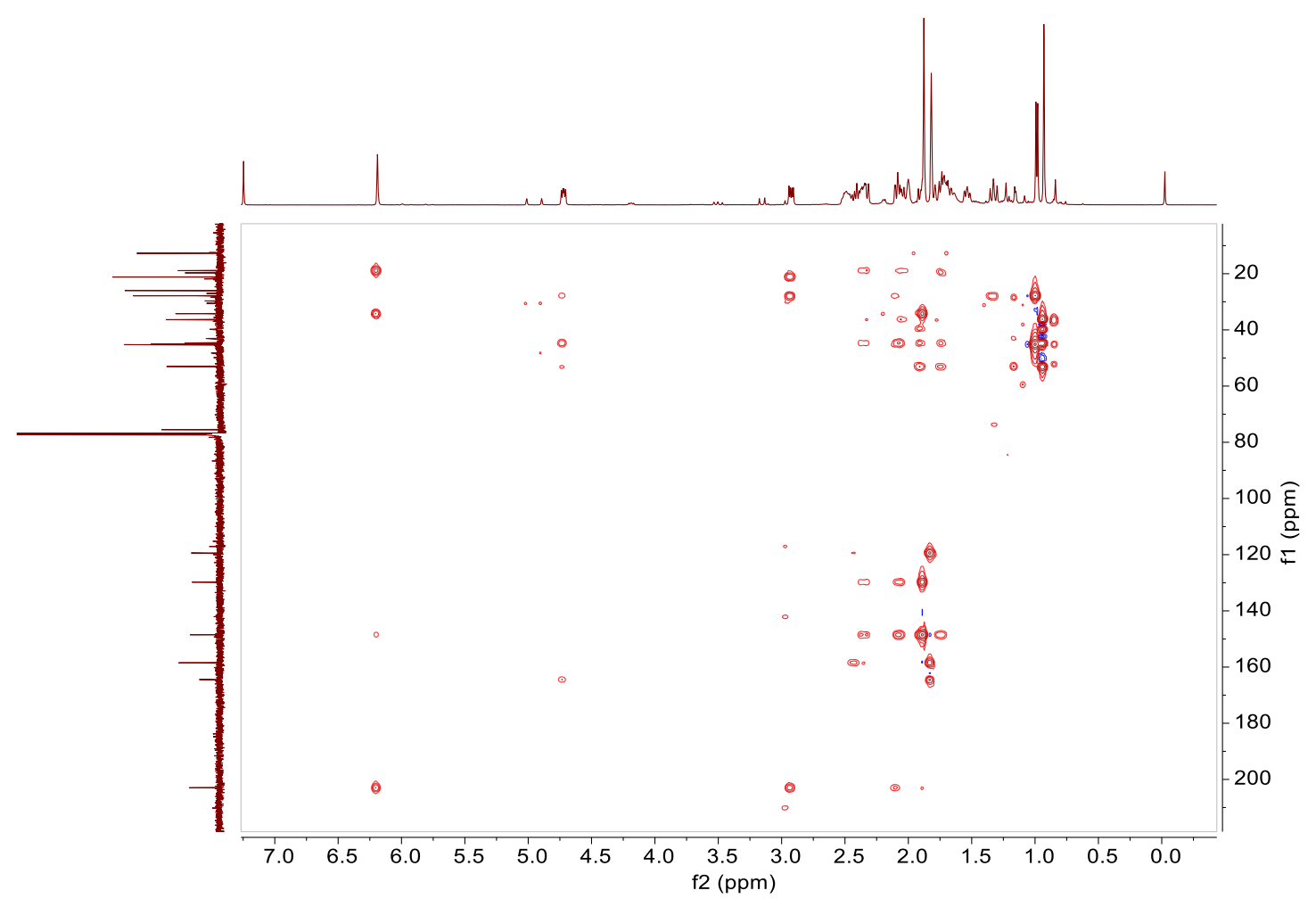

Figure S42. HMBC spectrum of compound 4 in $\mathrm{CDCl}_{3}$. 

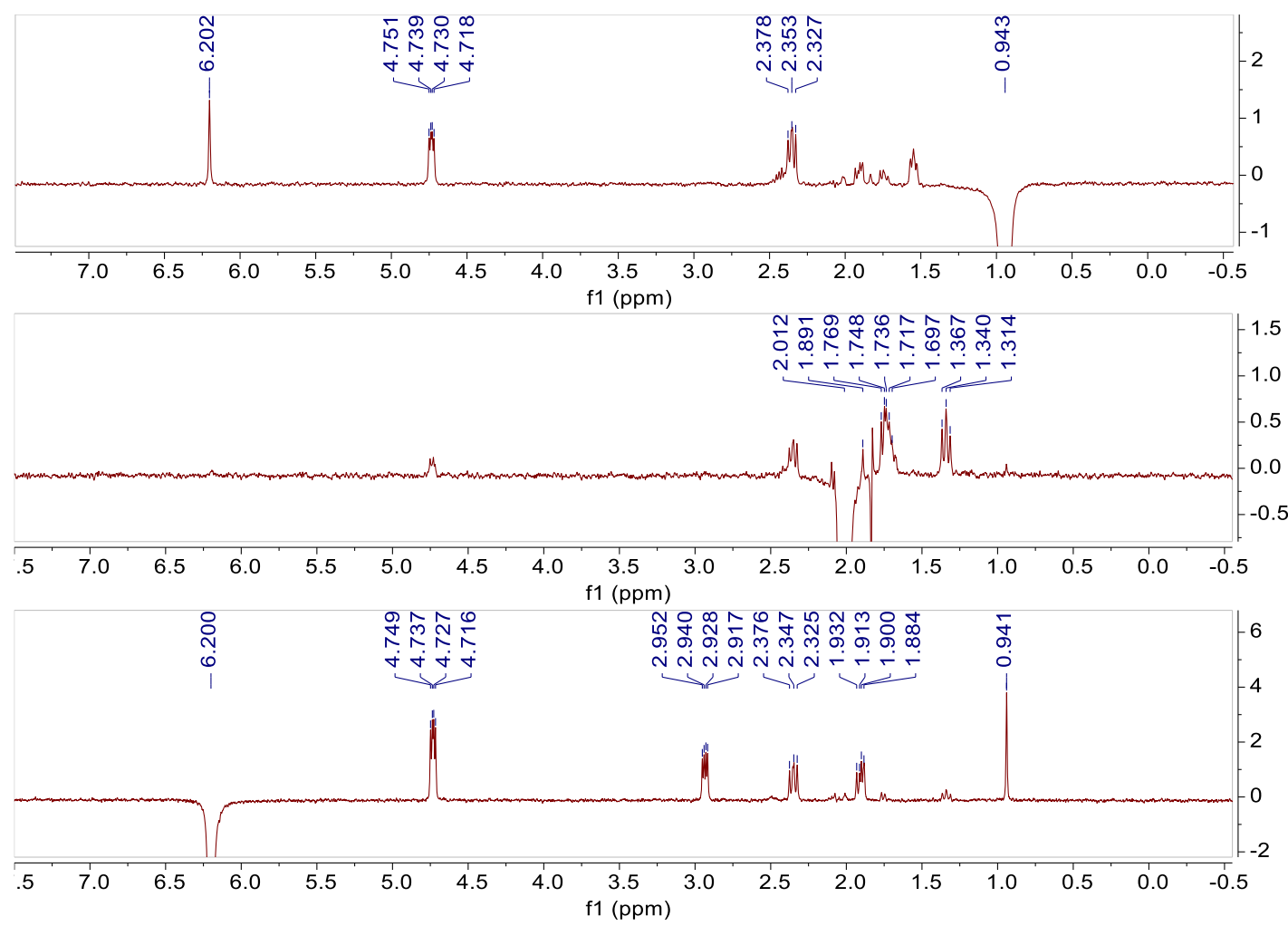

Figure S43. 1D NOE spectrum of compound 4 in $\mathrm{CDCl}_{3}$.

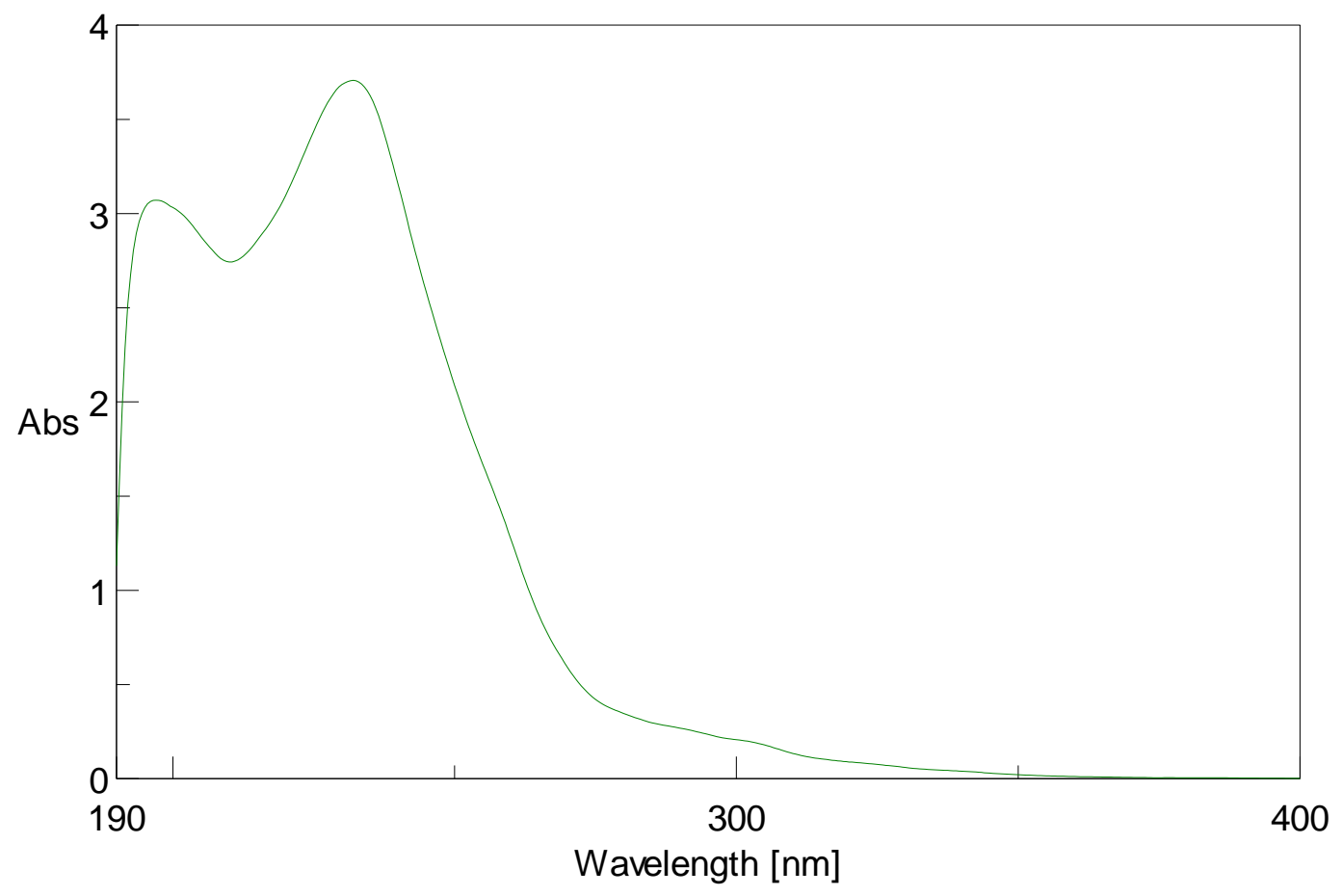

Figure S44. UV spectrum of compound 4. 


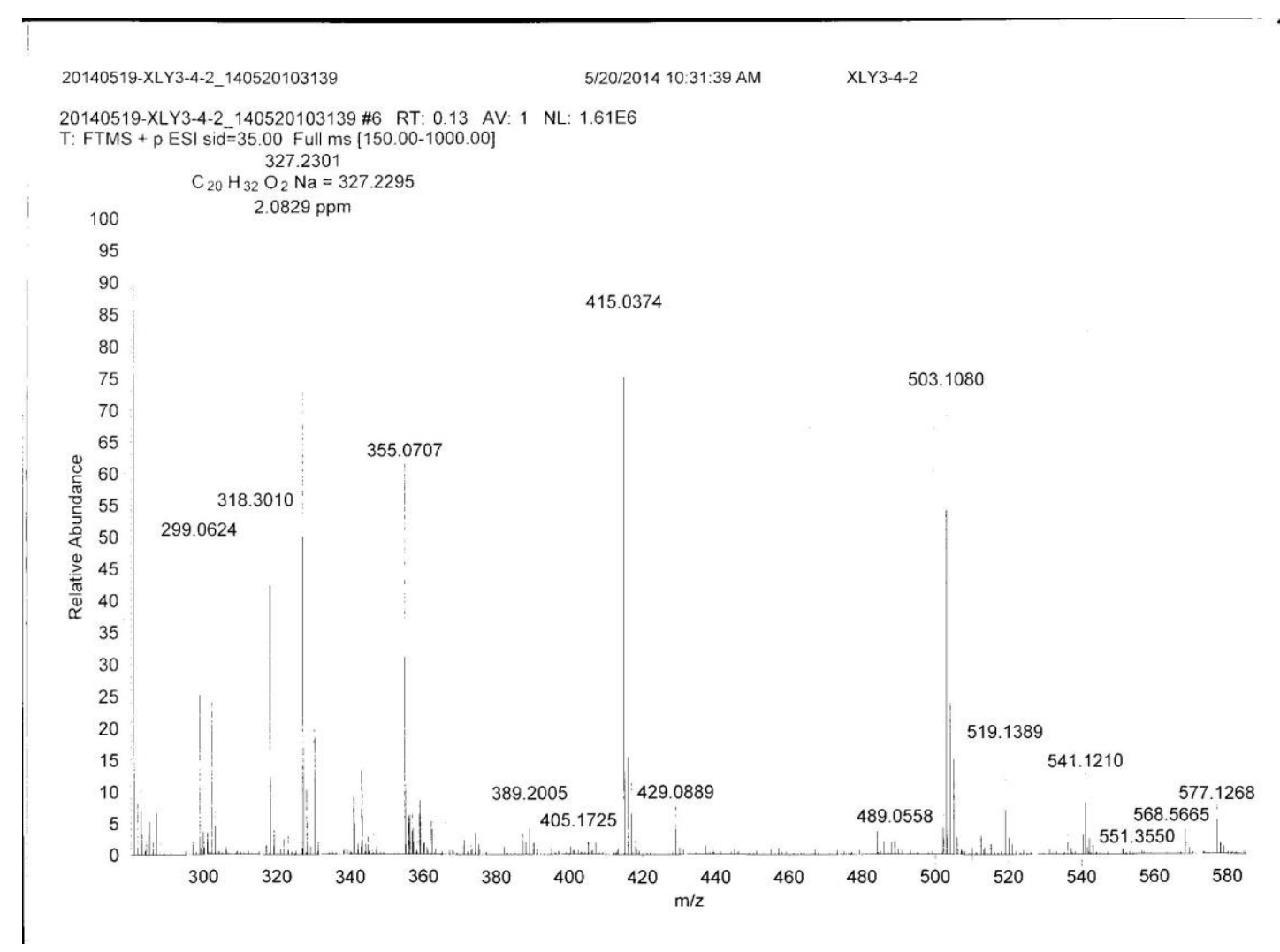

Figure S45. HRESIMS spectrum of compound $\mathbf{5}$.

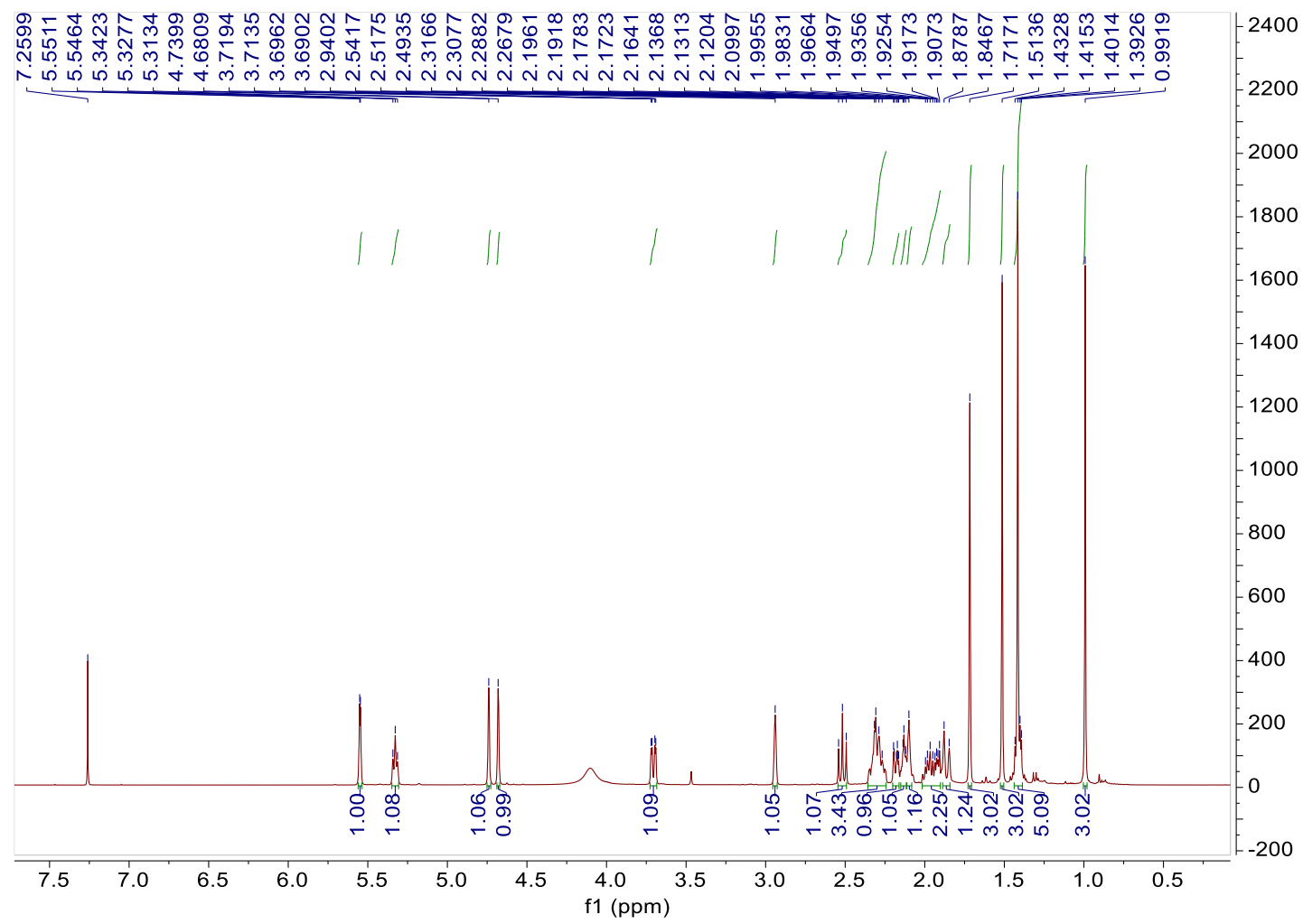

Figure S46. ${ }^{1} \mathrm{H}$ NMR spectrum of compound 5 in $\mathrm{CDCl}_{3}$. 


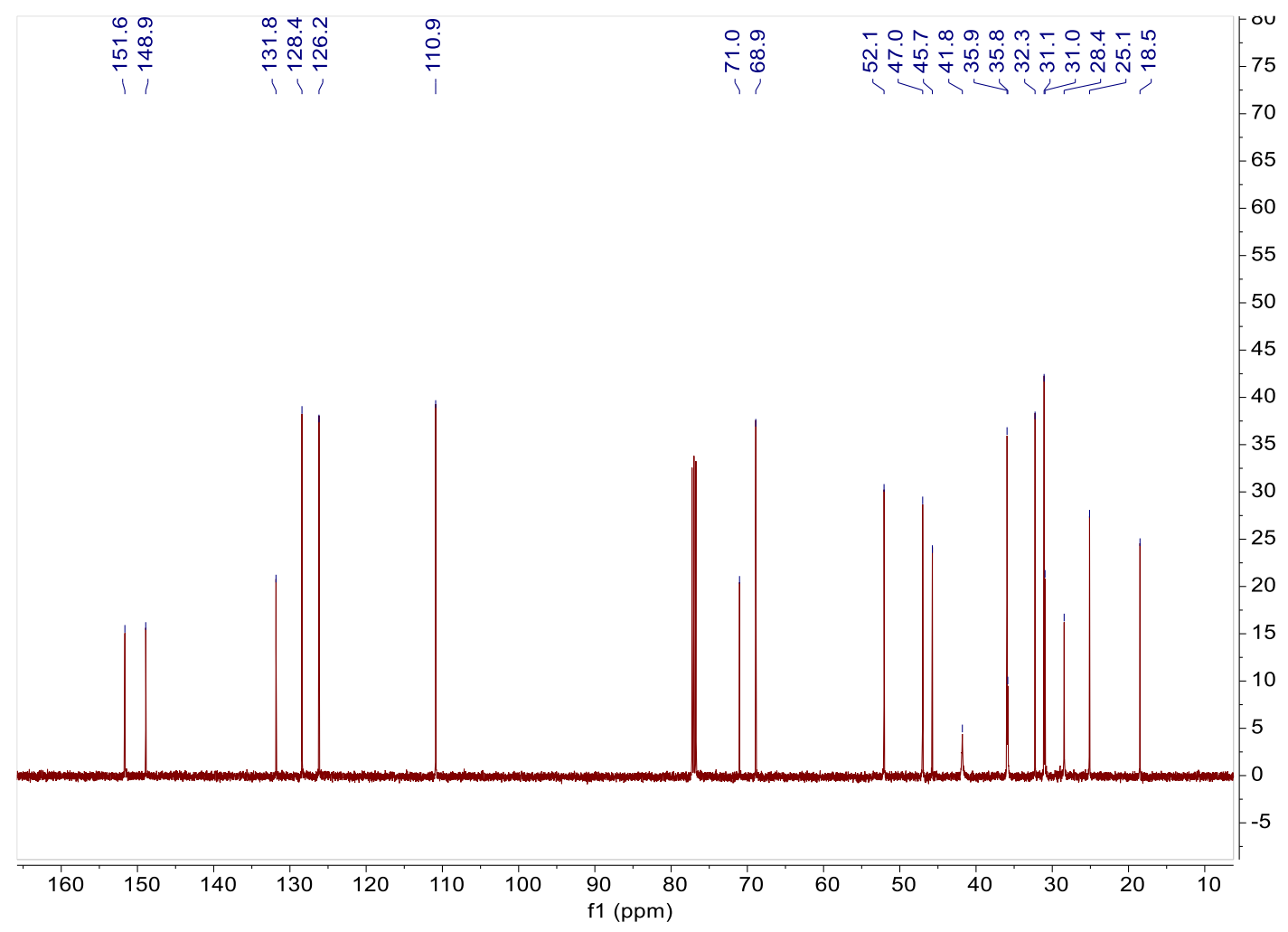

Figure S47. ${ }^{13} \mathrm{C}$ NMR spectrum of compound 5 in $\mathrm{CDCl}_{3}$.

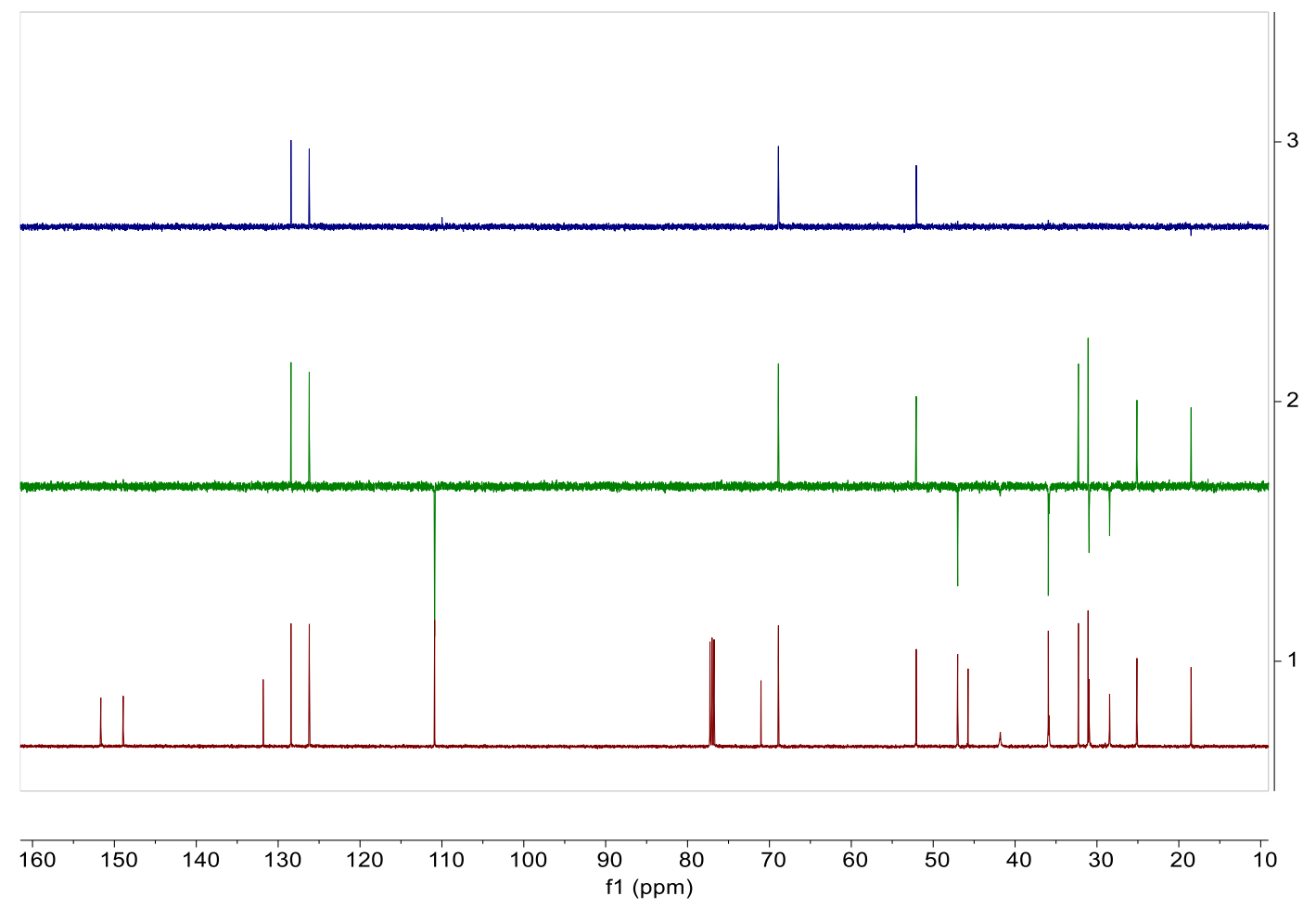

Figure S48. ${ }^{13} \mathrm{C}$ and DEPT spectrum of compound 5 in $\mathrm{CDCl}_{3}$. 


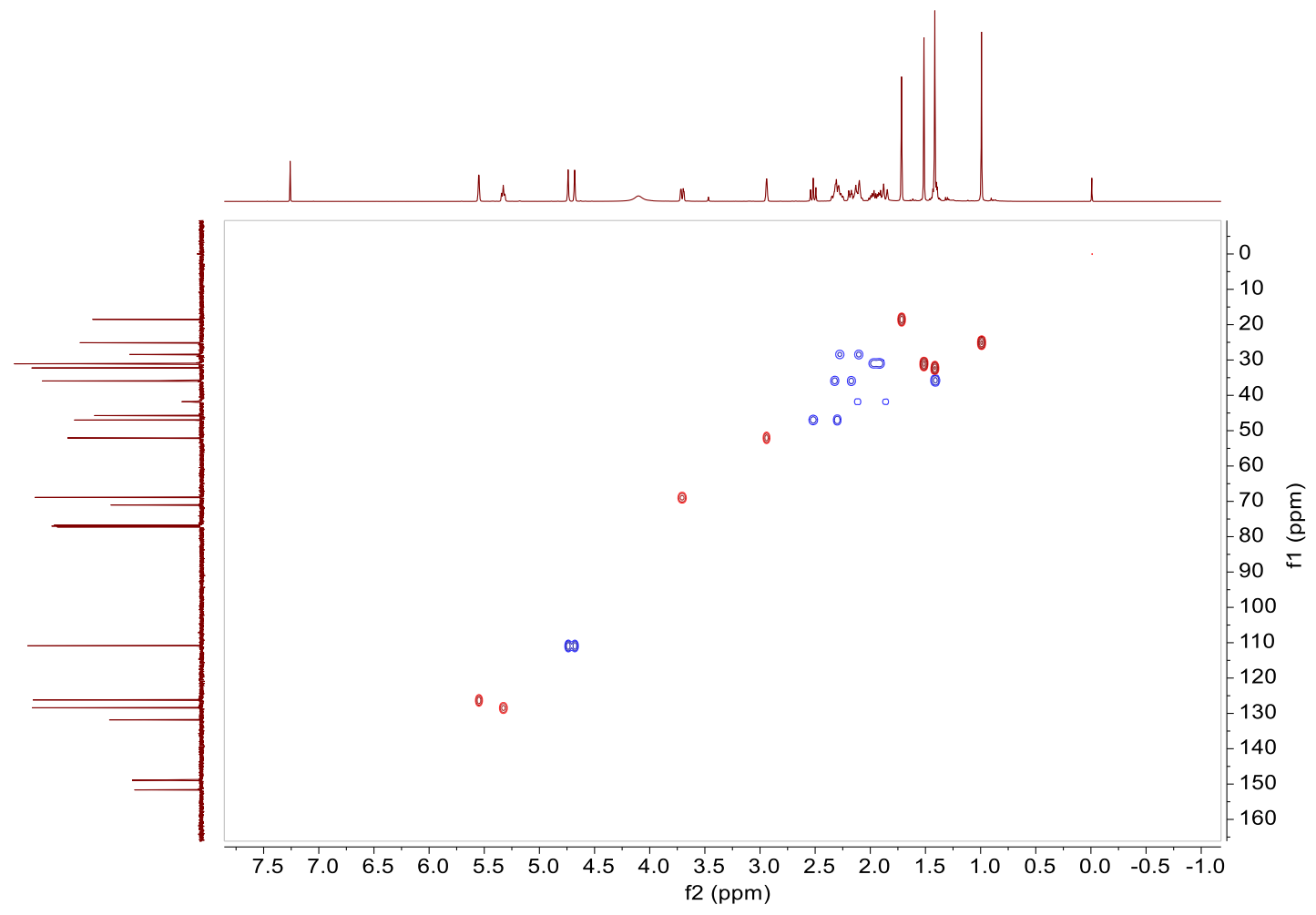

Figure S49. HSQC spectrum of compound 5 in $\mathrm{CDCl}_{3}$.

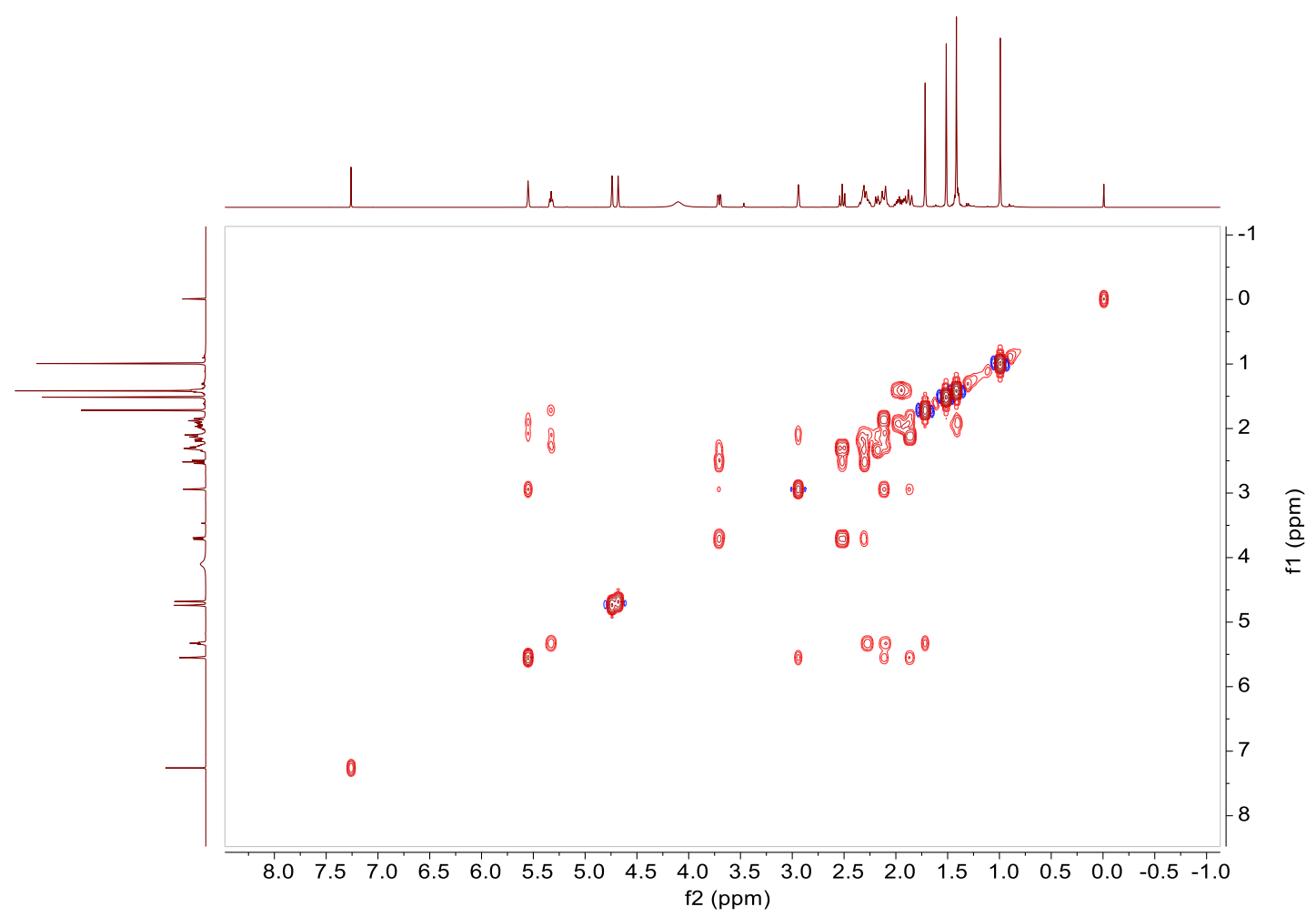

Figure S50. ${ }^{1} \mathrm{H}-{ }^{1} \mathrm{H}-\mathrm{COSY}$ spectrum of compound $\mathbf{5}$ in $\mathrm{CDCl}_{3}$. 


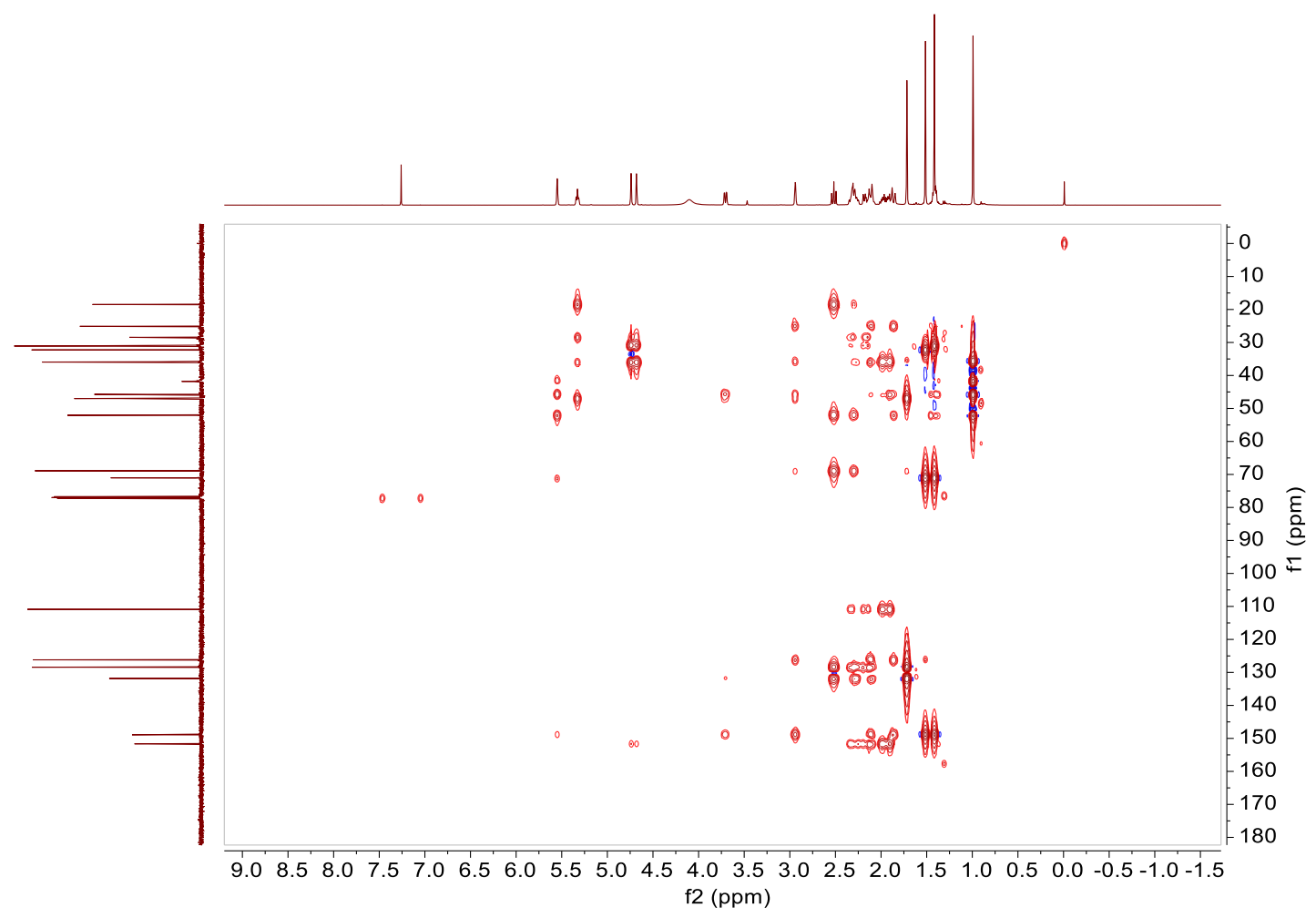

Figure S51. HMBC spectrum of compound 5 in $\mathrm{CDCl}_{3}$.

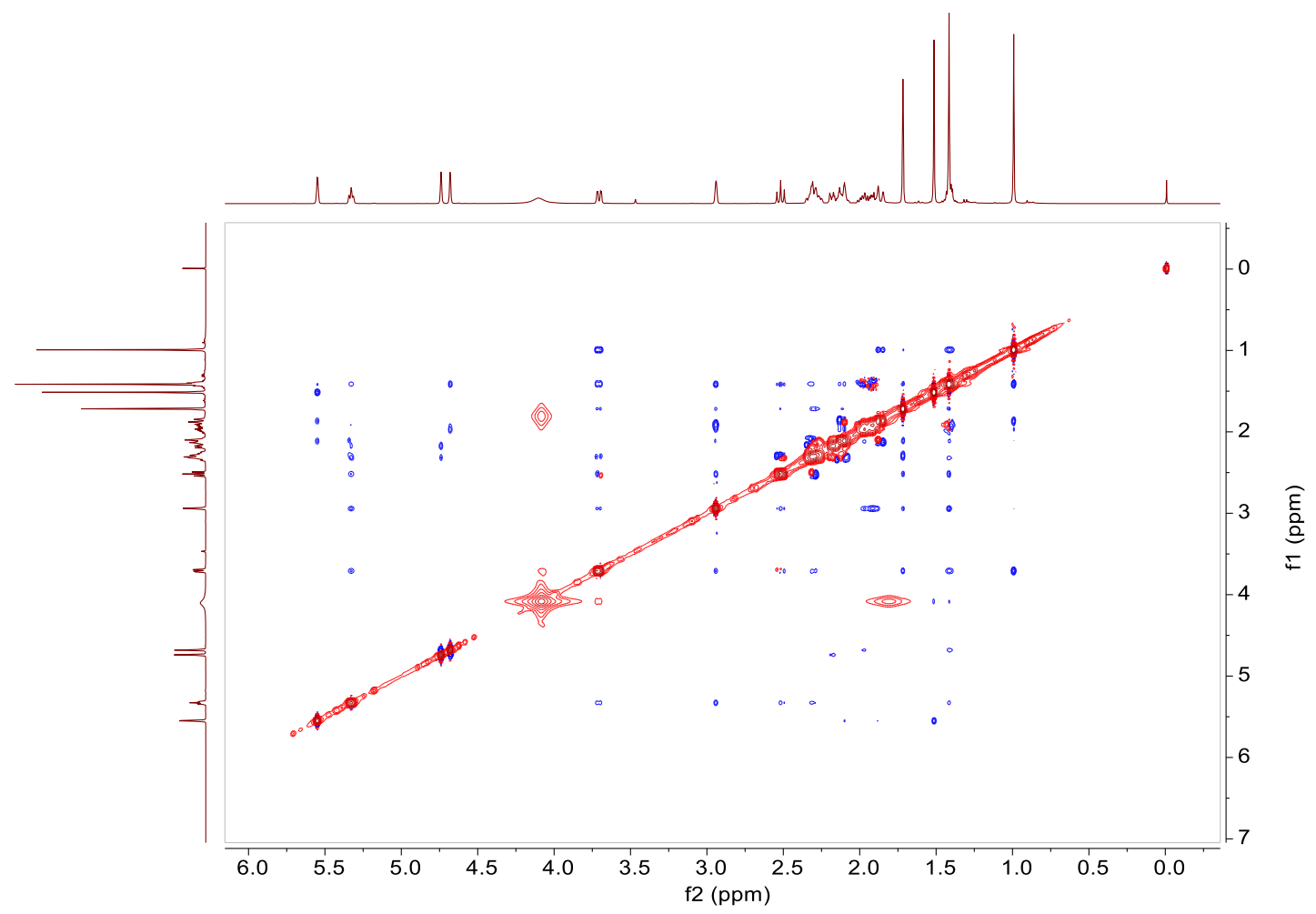

Figure S52. NOESY spectrum of compound 5 in $\mathrm{CDCl}_{3}$. 


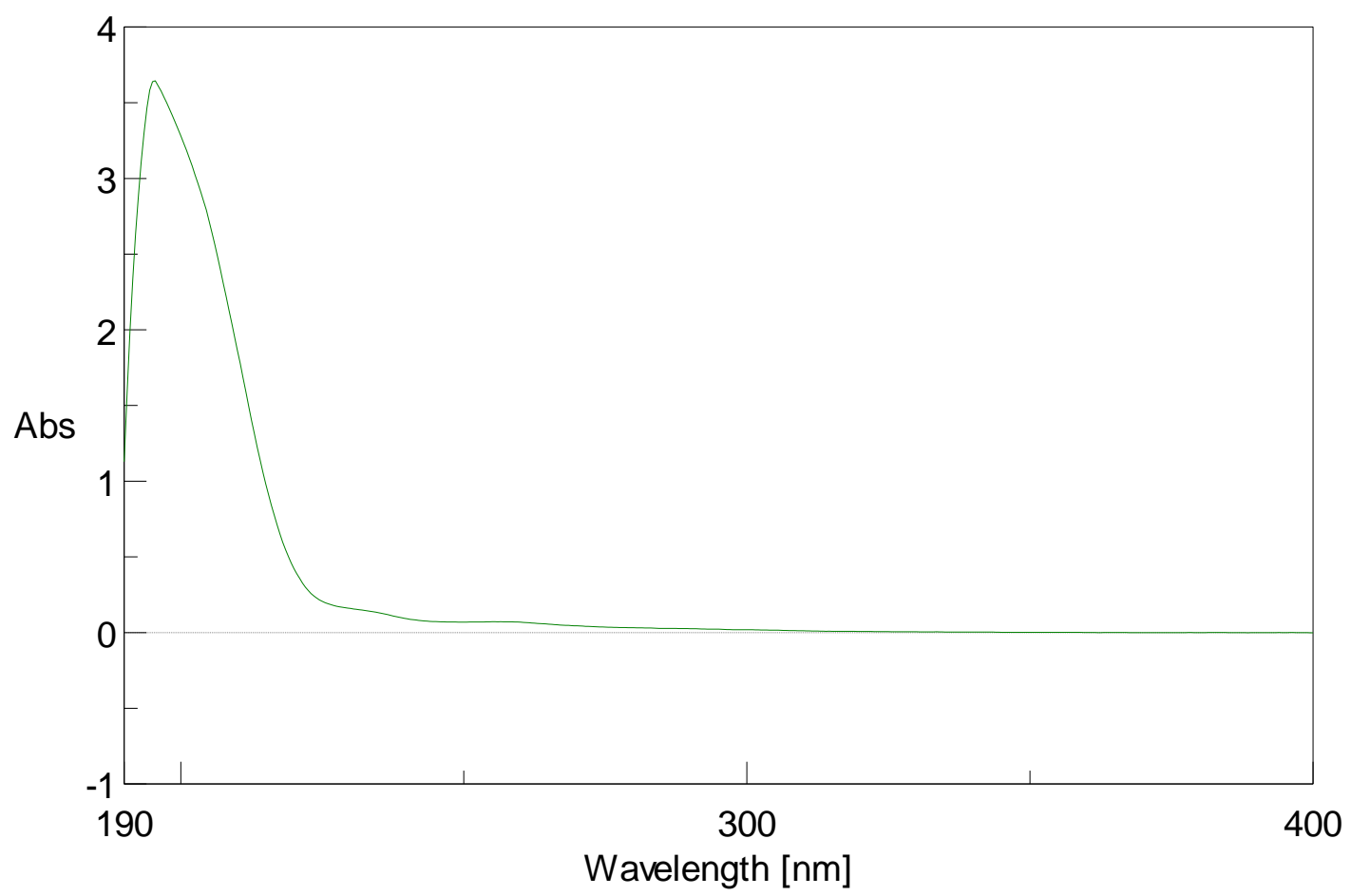

Figure S53. UV spectrum of compound 5.

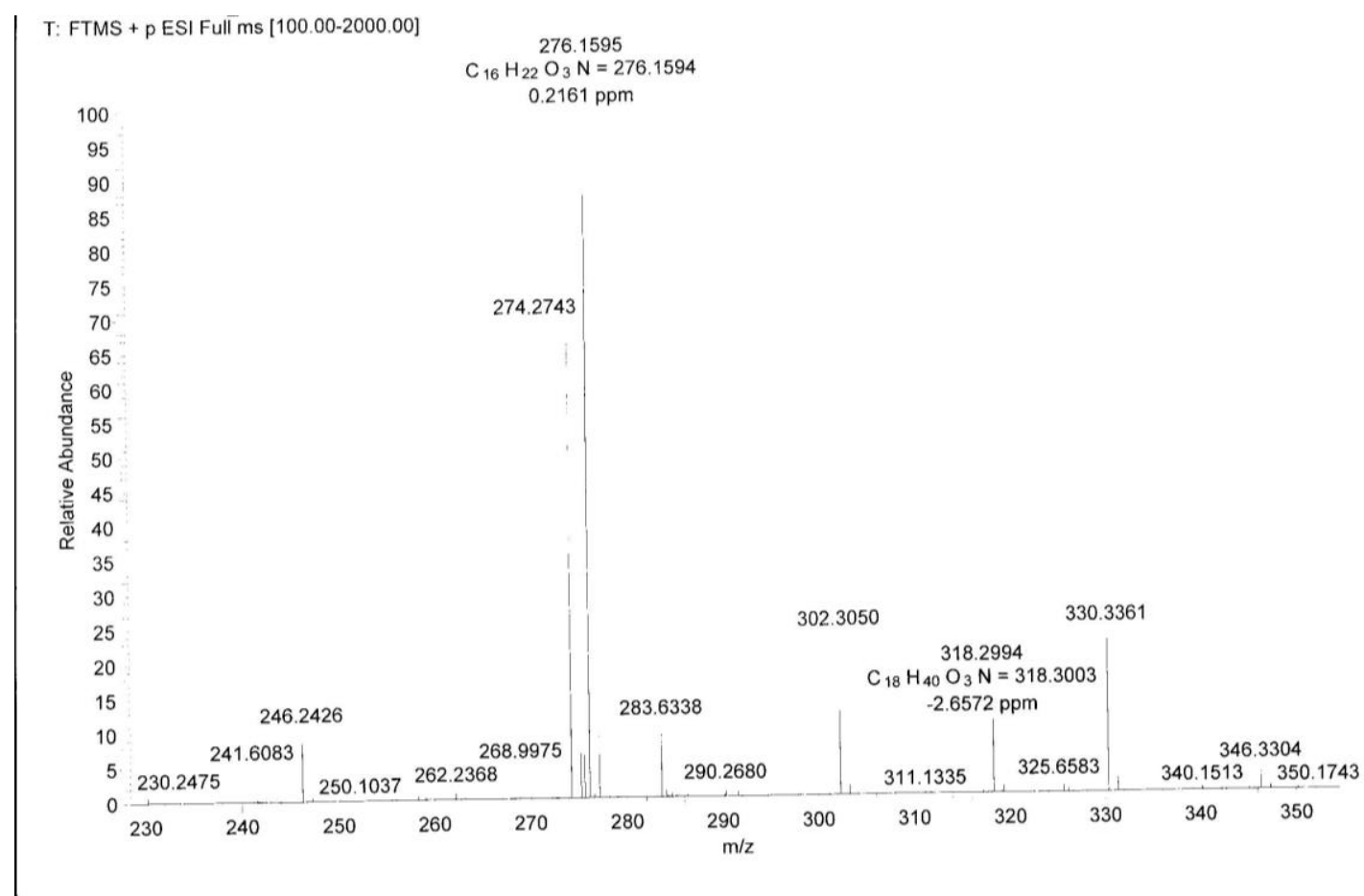

Figure S54. HRESIMS spectrum of compound 7. 


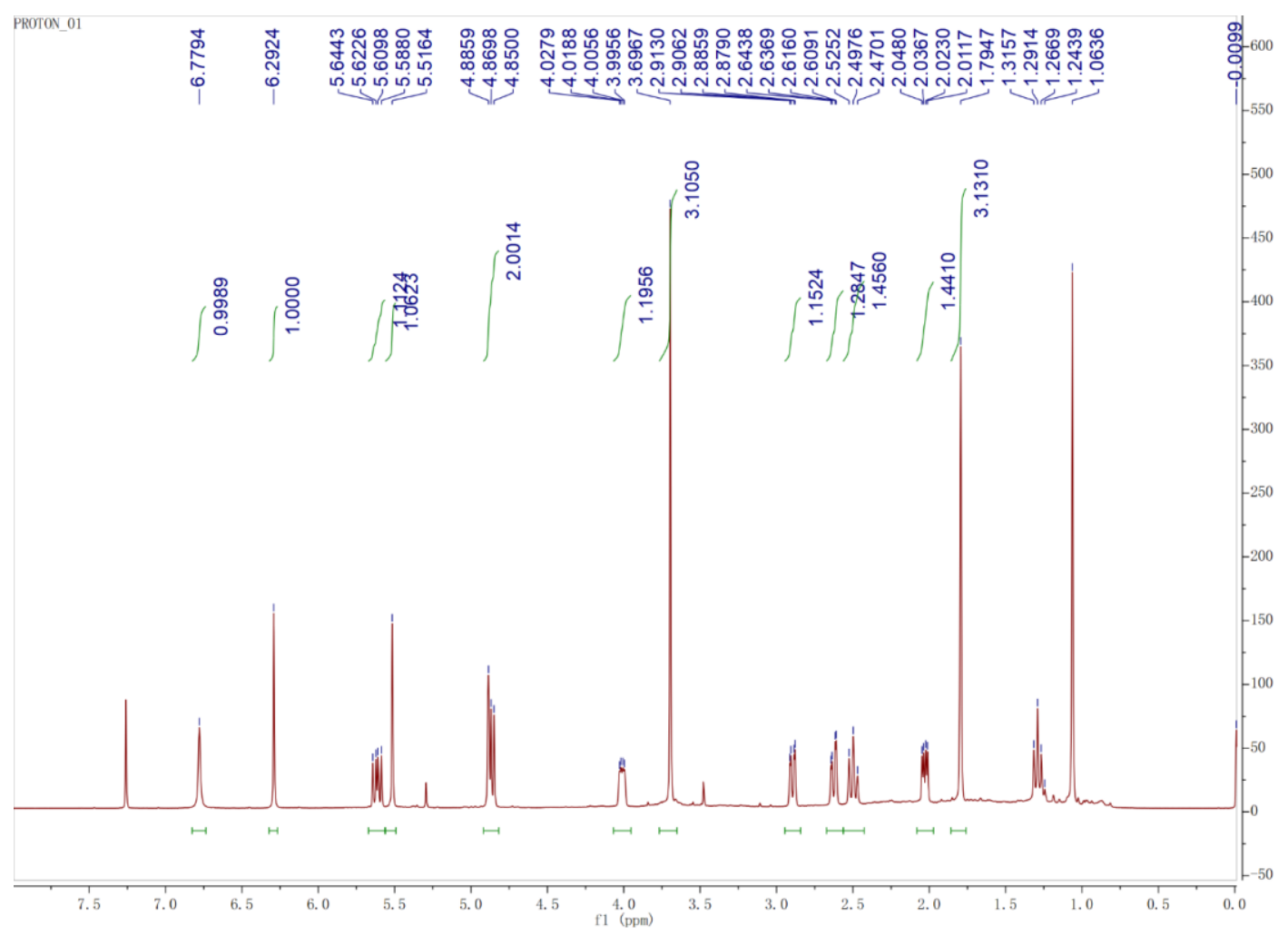

Figure S55. ${ }^{1} \mathrm{H}$ NMR spectrum of compound 7 in $\mathrm{CDCl}_{3}$.
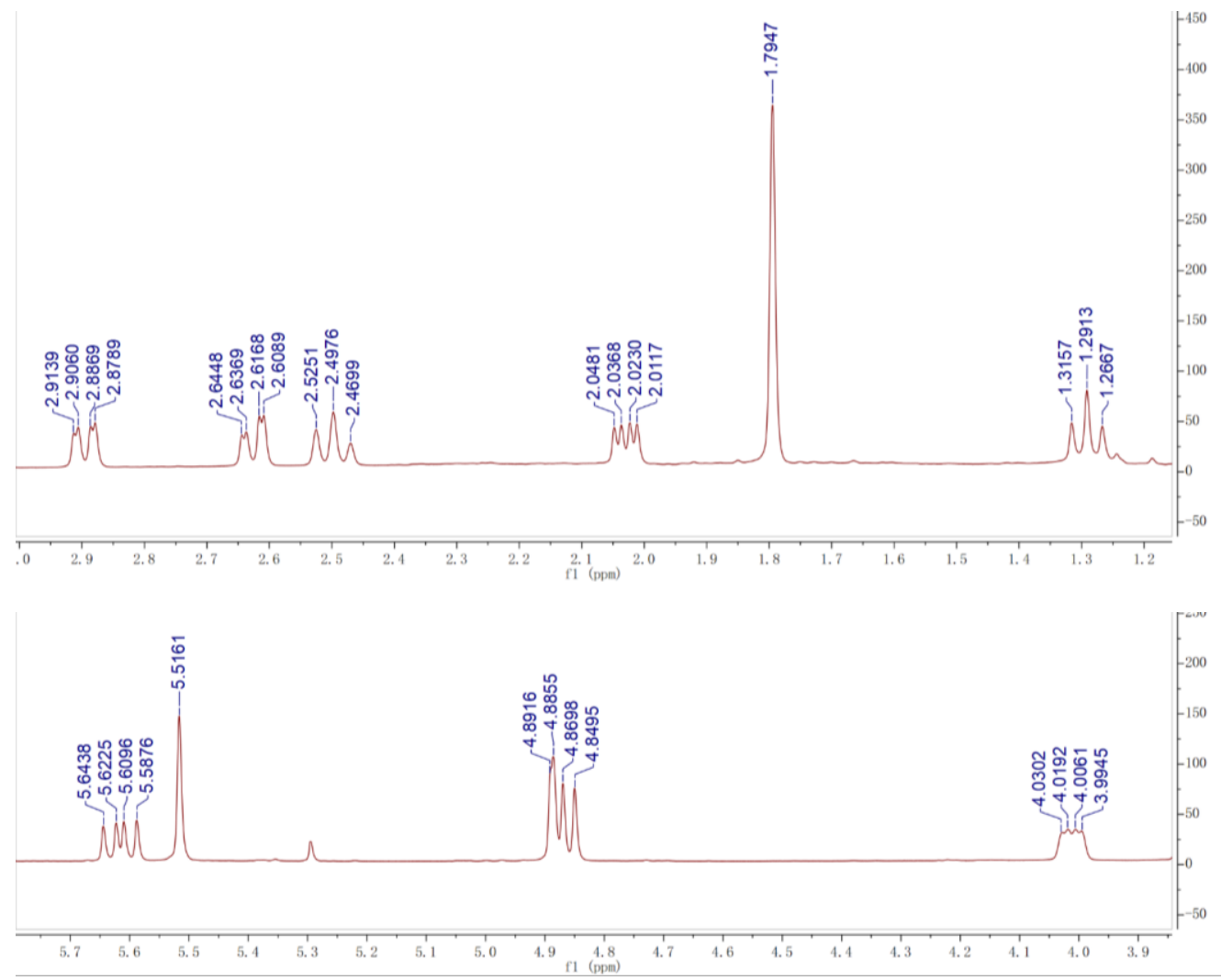

Figure S56. The amplificatory ${ }^{1} \mathrm{H}$ NMR spectrum of compound 7 in $\mathrm{CDCl}_{3}$. 


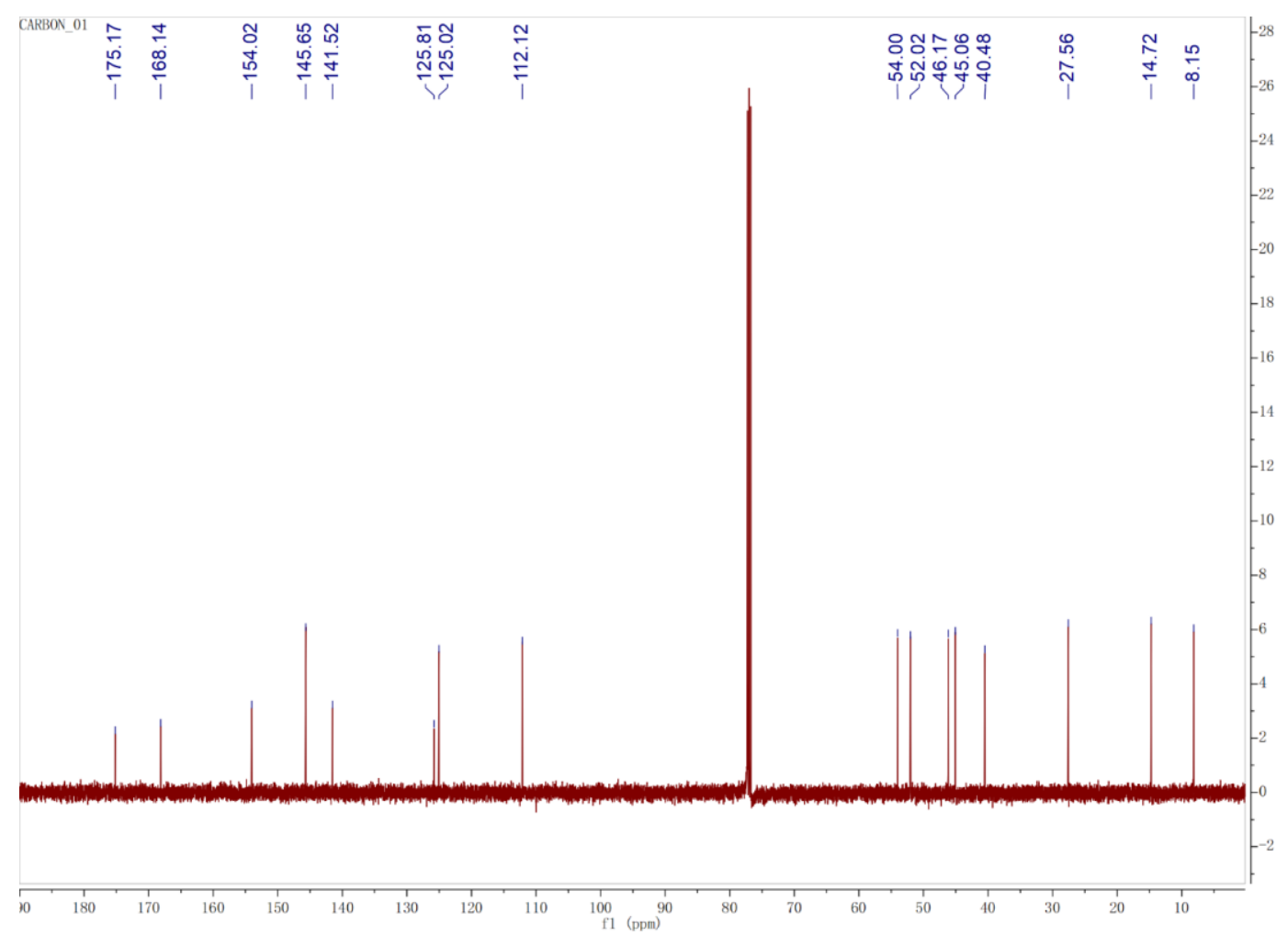

Figure S57. ${ }^{13} \mathrm{C}$ NMR spectrum of compound 7 in $\mathrm{CDCl}_{3}$.

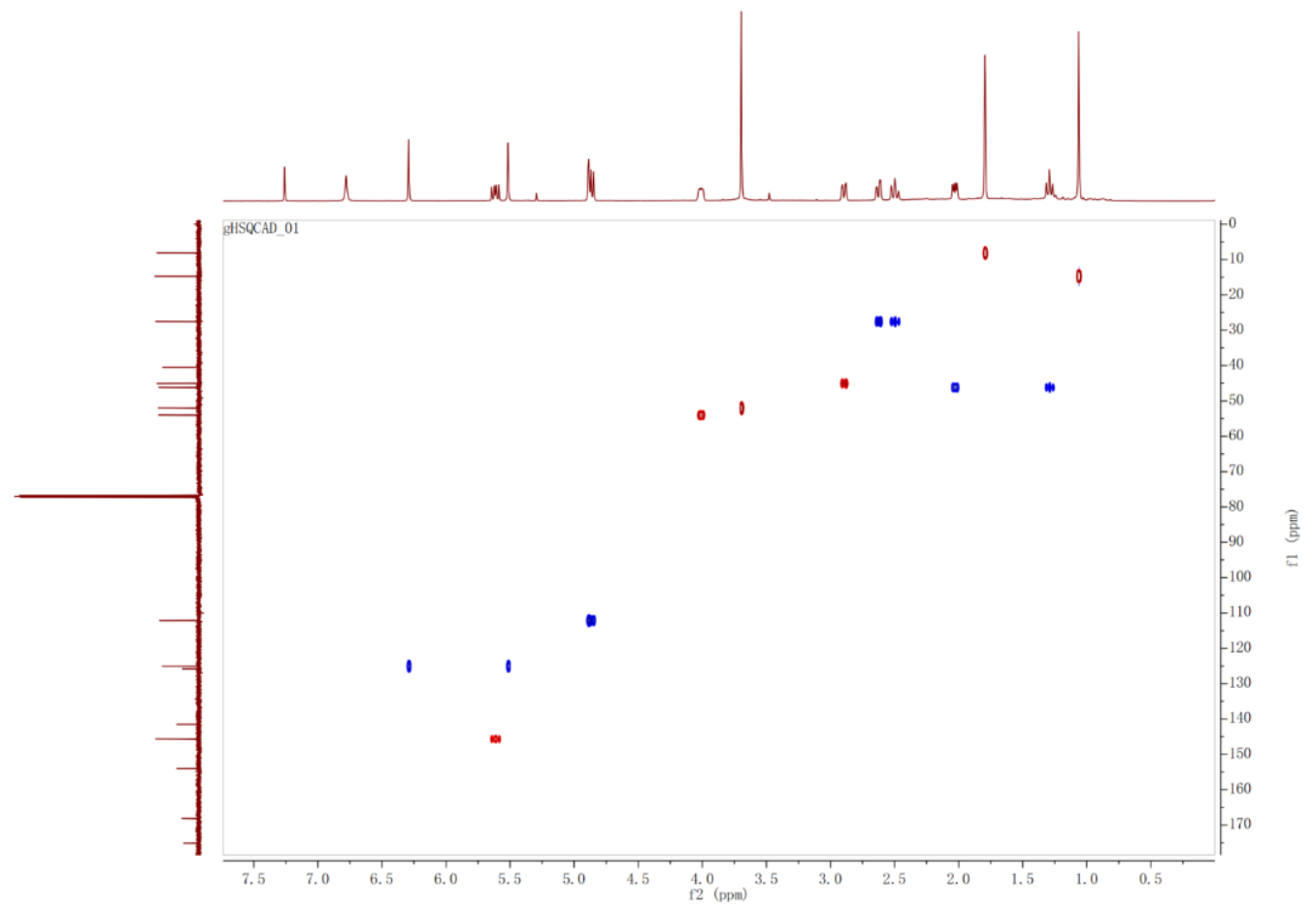

Figure S58. HSQC spectrum of compound 7 in $\mathrm{CDCl}_{3}$. 


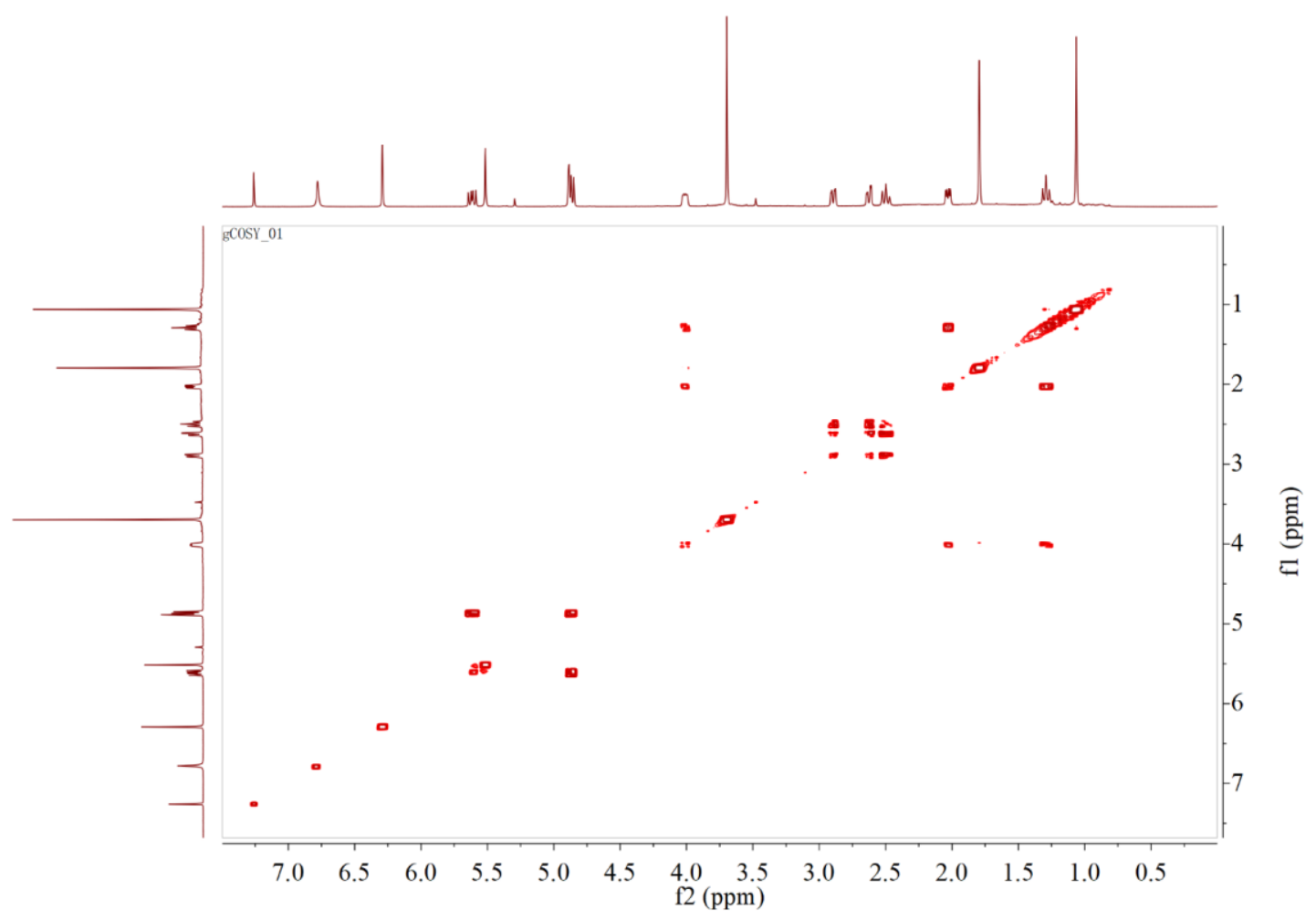

Figure S59. ${ }^{1} \mathrm{H}-{ }^{1} \mathrm{H}$ COSY spectrum of compound 7 in $\mathrm{CDCl} 3$.

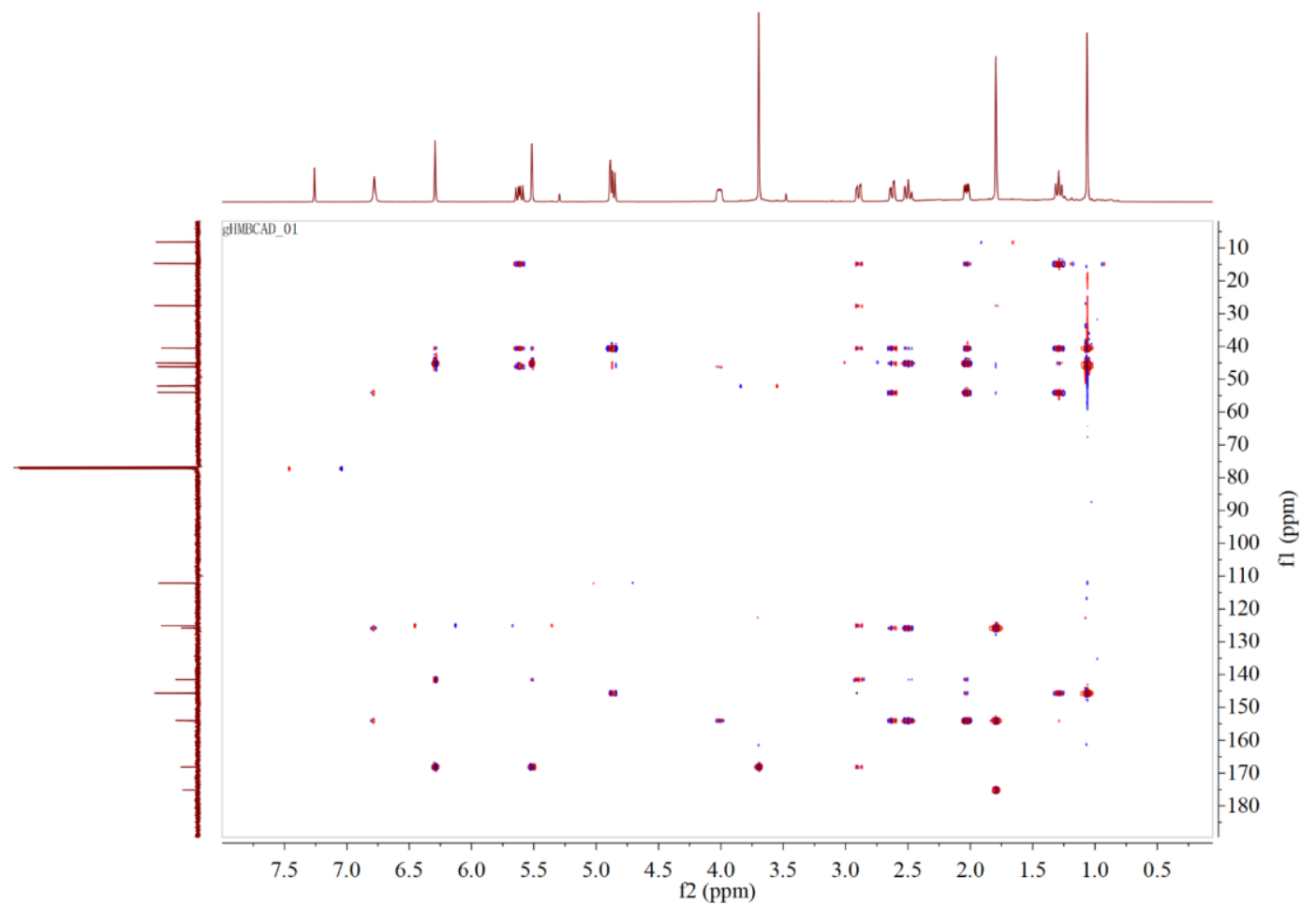

Figure S60. $\mathrm{HMBC}$ spectrum of compound 7 in $\mathrm{CDCl}_{3}$. 


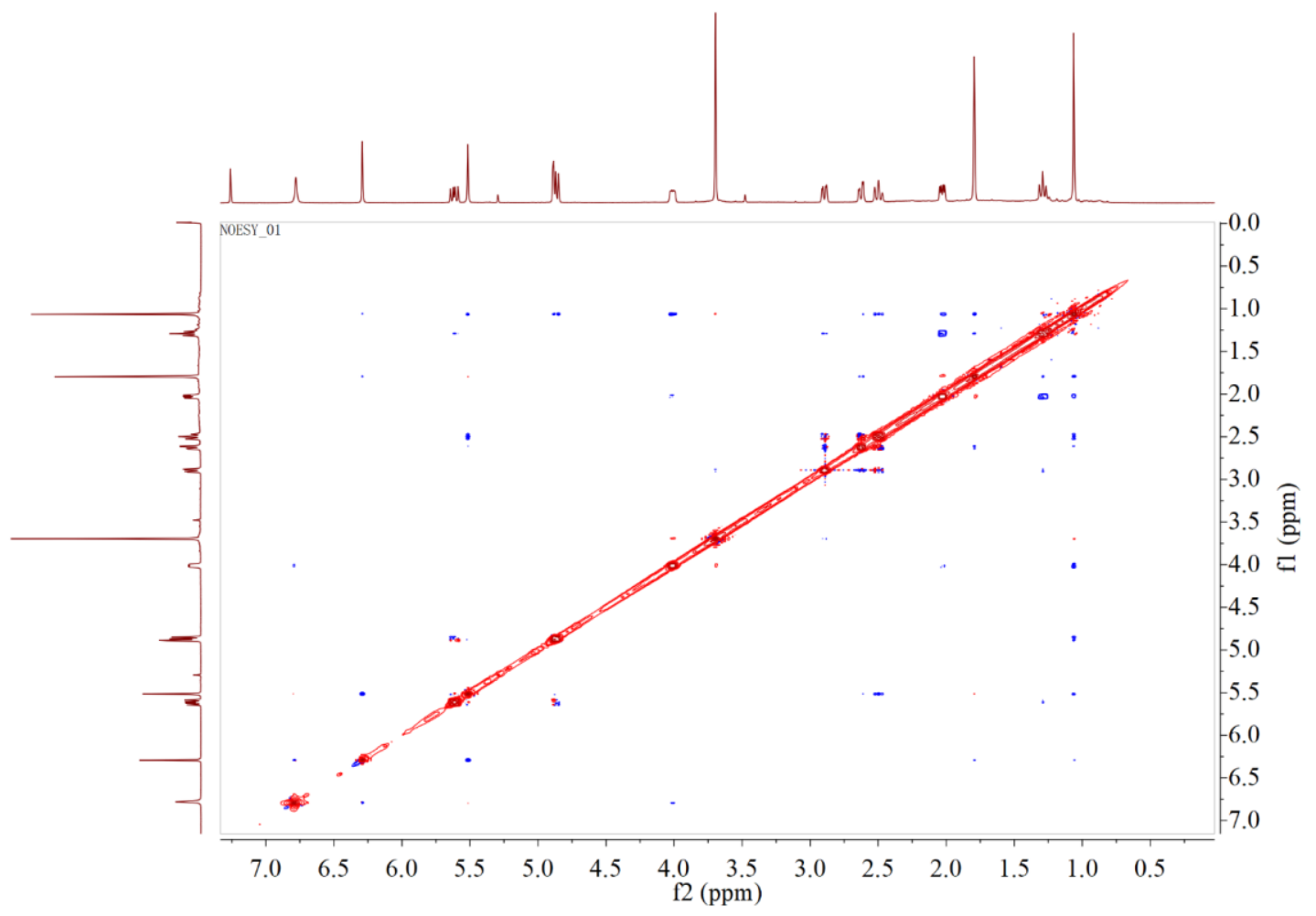

Figure S61. NOESY spectrum of compound 7 in $\mathrm{CDCl}_{3}$.

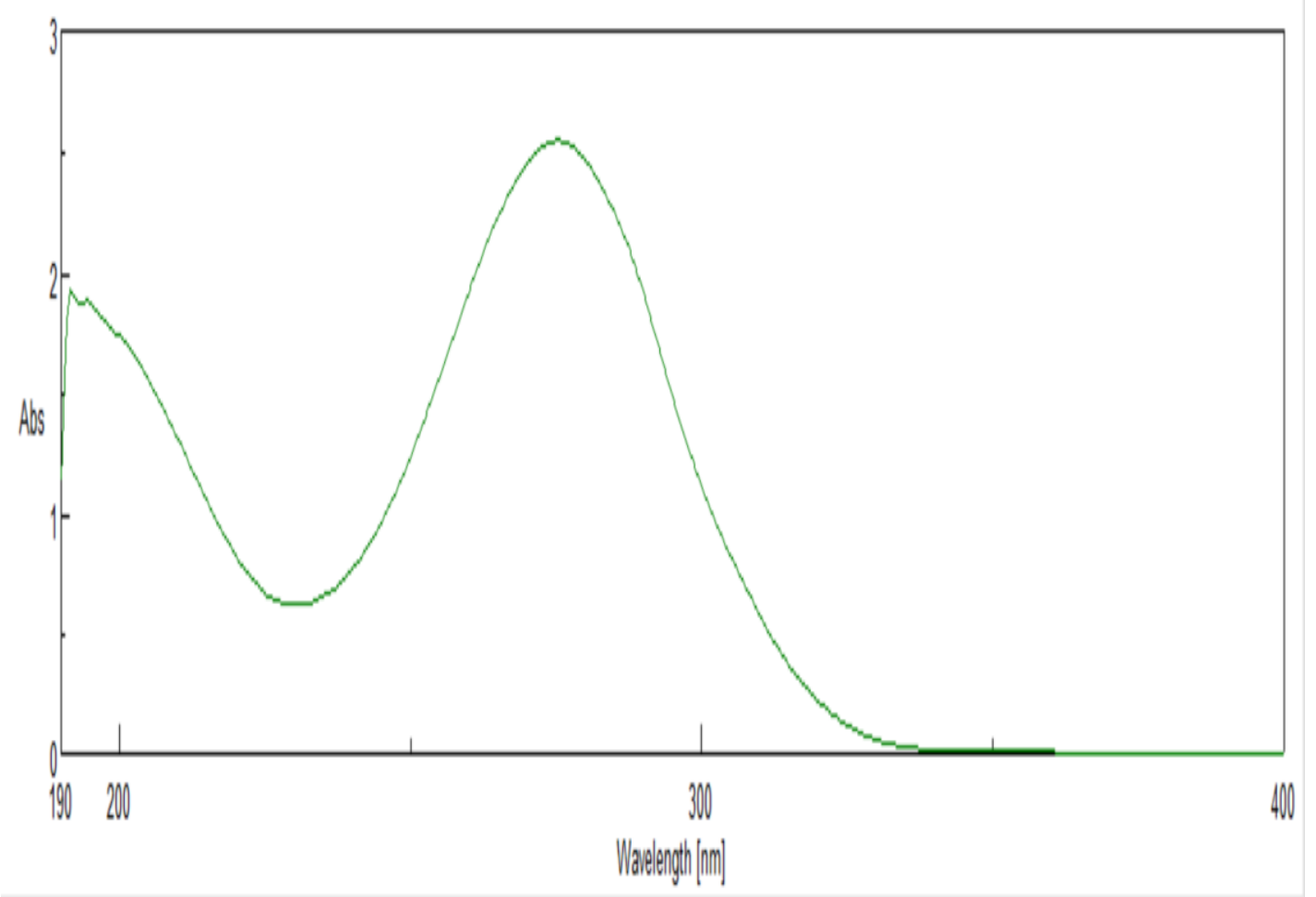

Figure S62. UV spectrum of compound 7. 


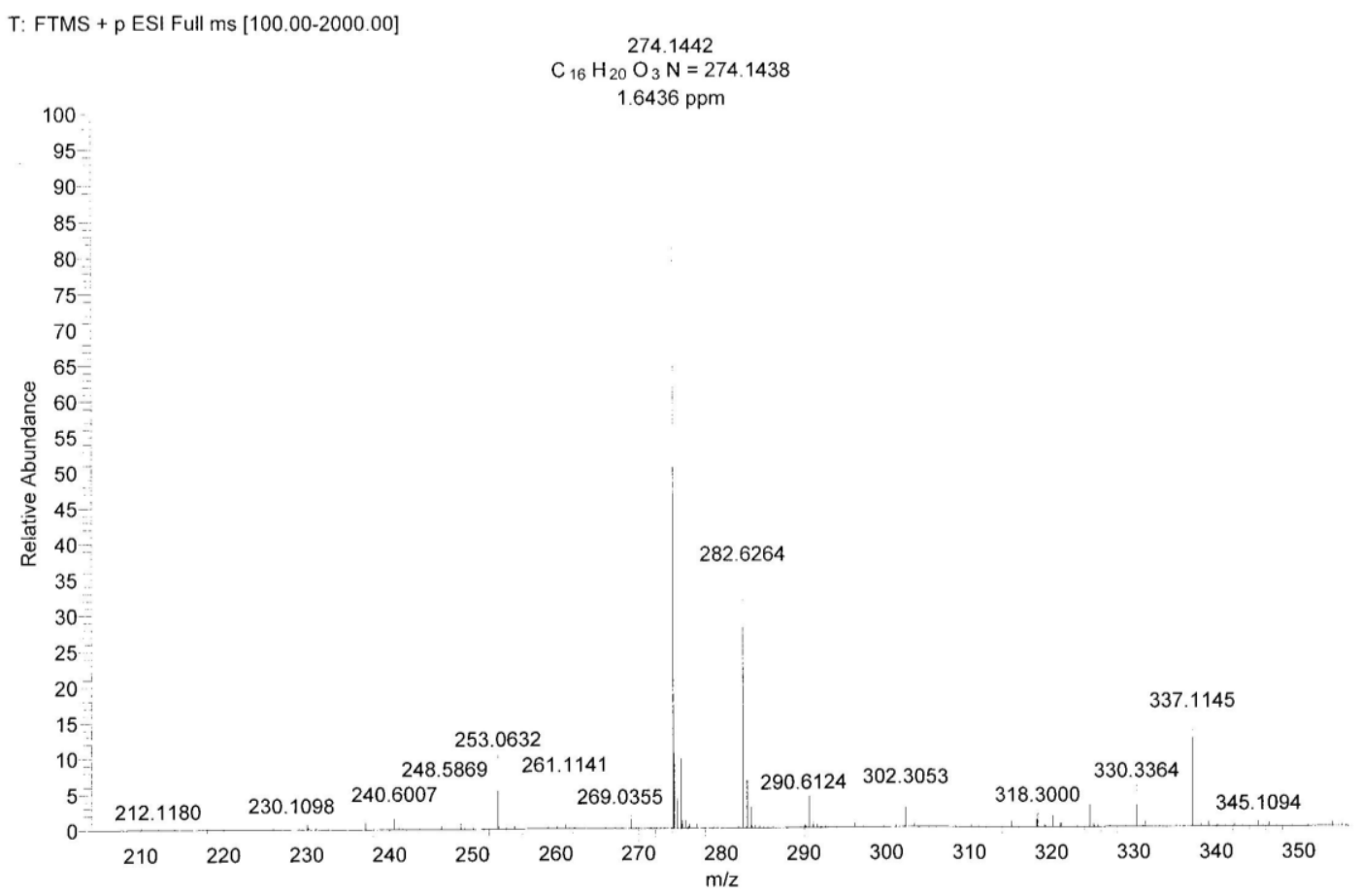

Figure S63. HRESIMS spectrum of compound 8.

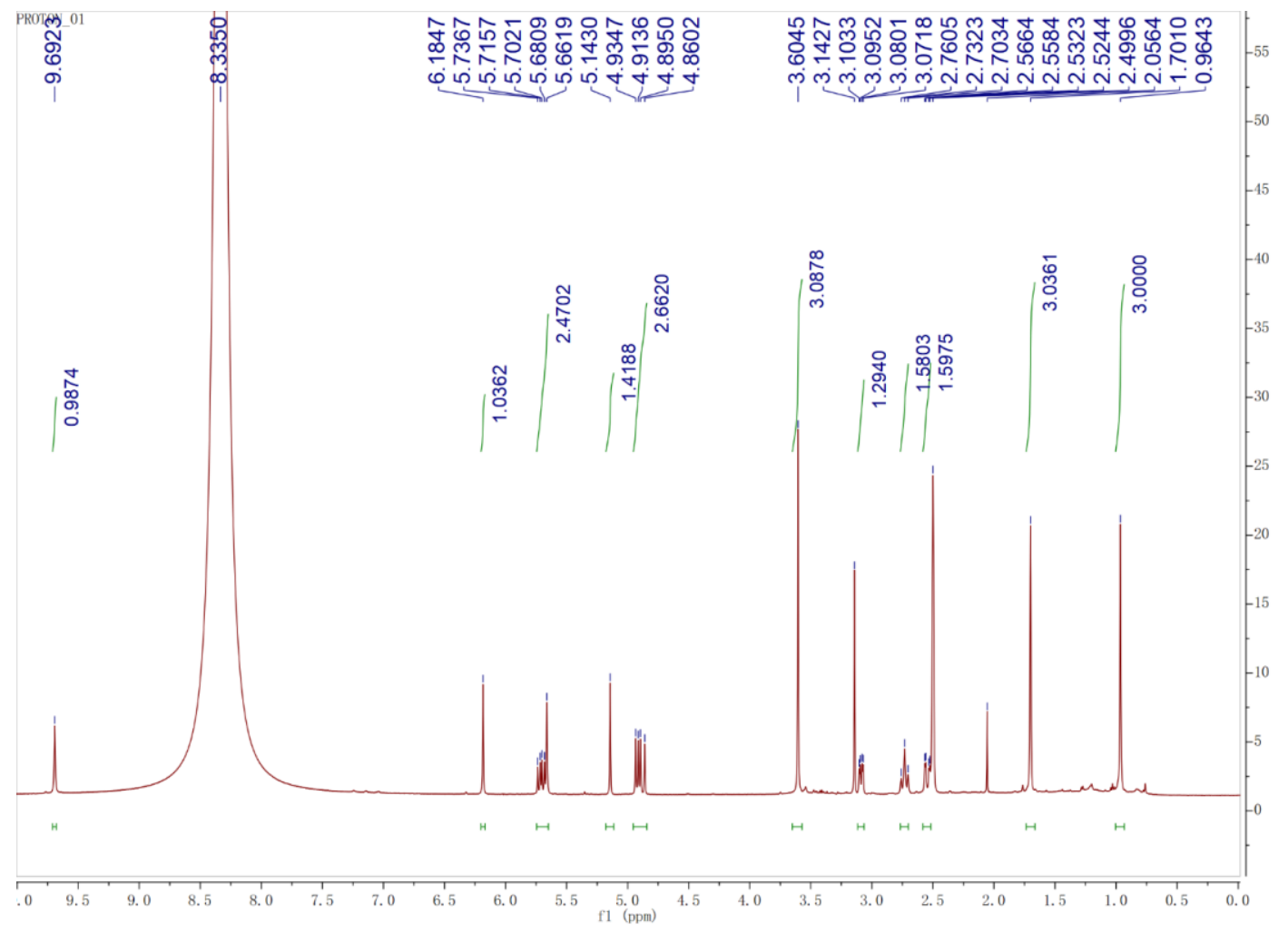

Figure S64. ${ }^{1} \mathrm{H}$ NMR spectrum of compound 8 in DMSO- $d 6$. 


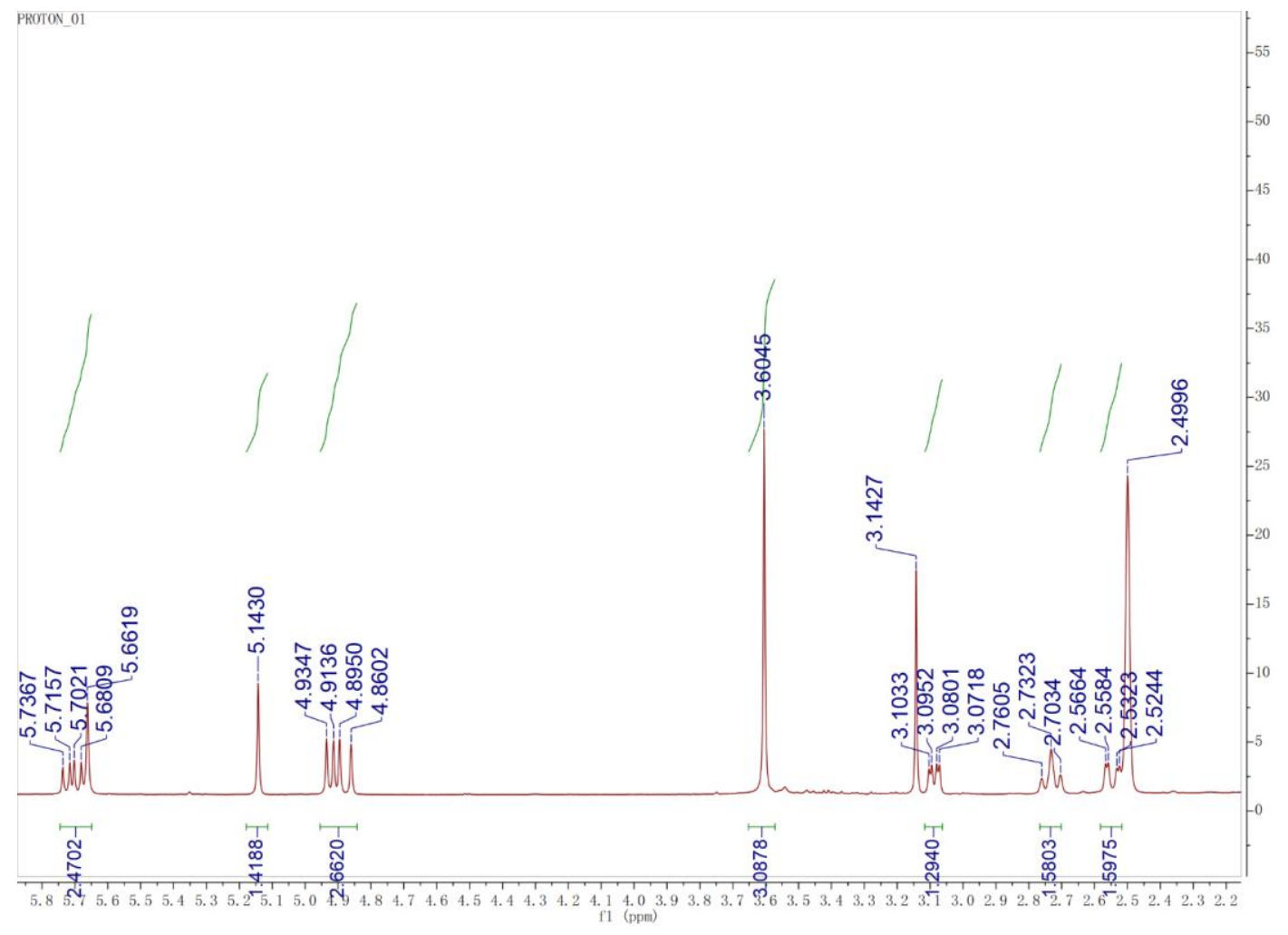

Figure S65. The amplificatory ${ }^{1} \mathrm{H}$ NMR spectrum of compound 8 in DMSO- $d 6$.

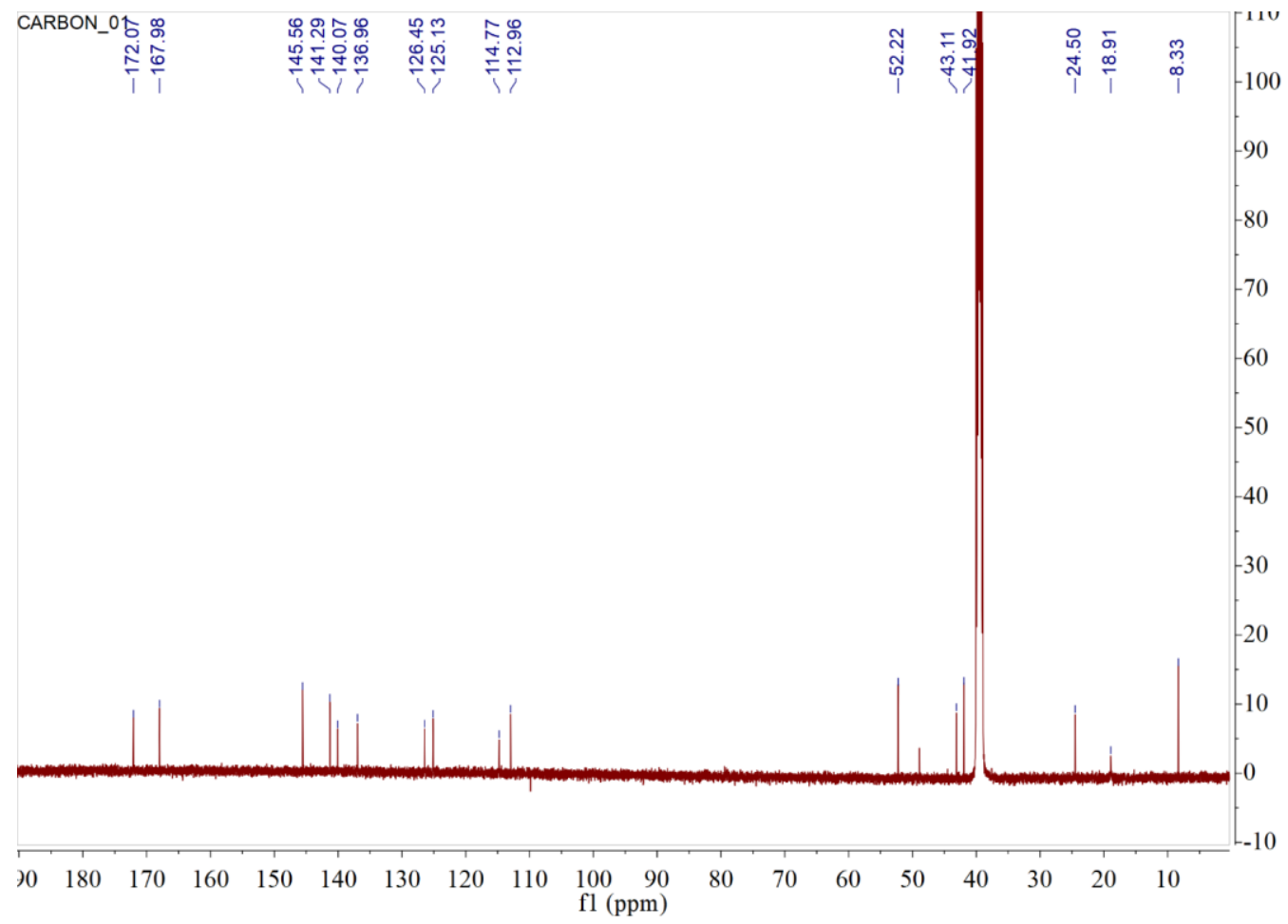

Figure S66. ${ }^{13} \mathrm{C}$ NMR spectrum of compound 8 in DMSO- $d_{6}$. 


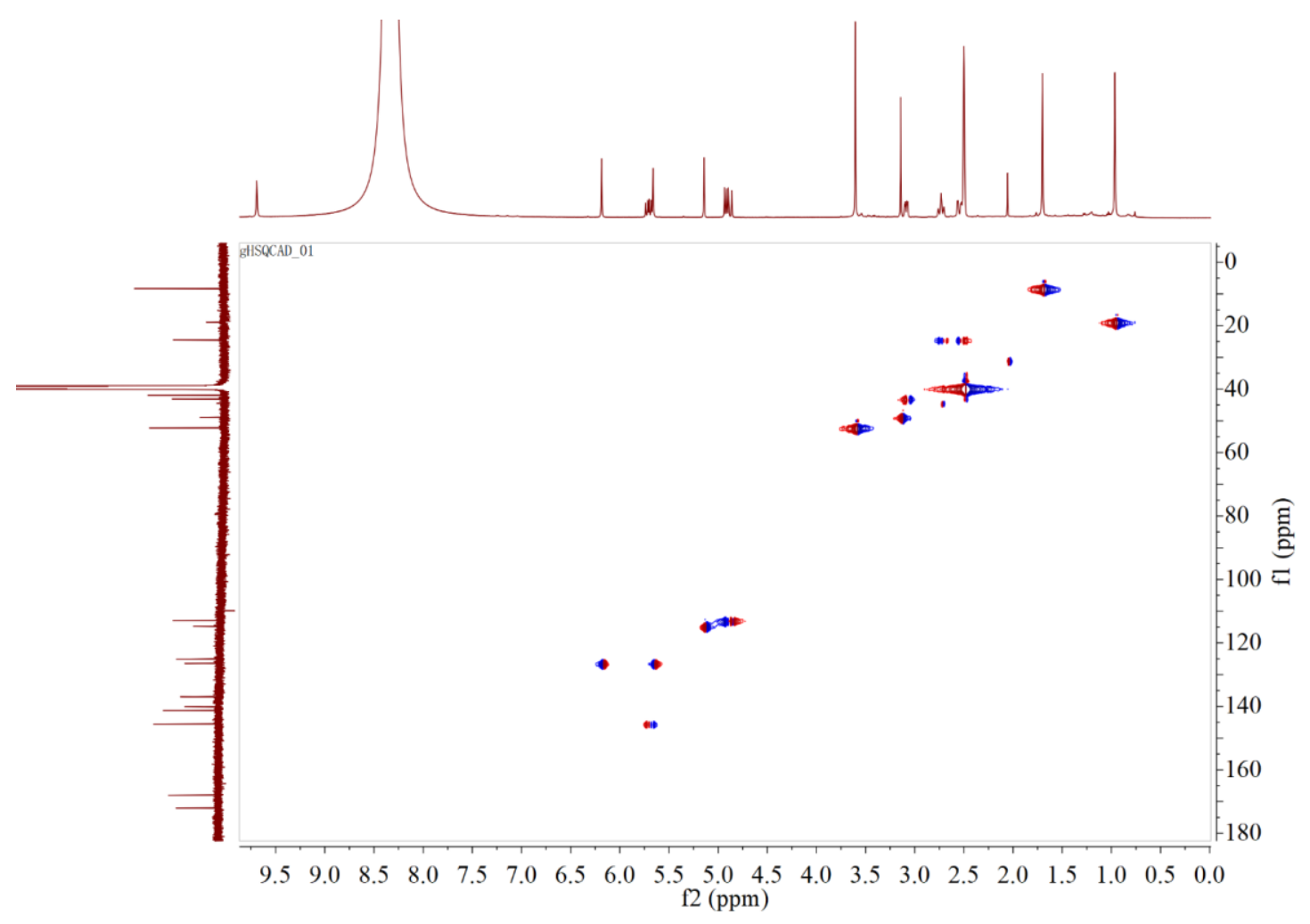

Figure S67. HSQC spectrum of compound 8 in DMSO- $d_{6}$.

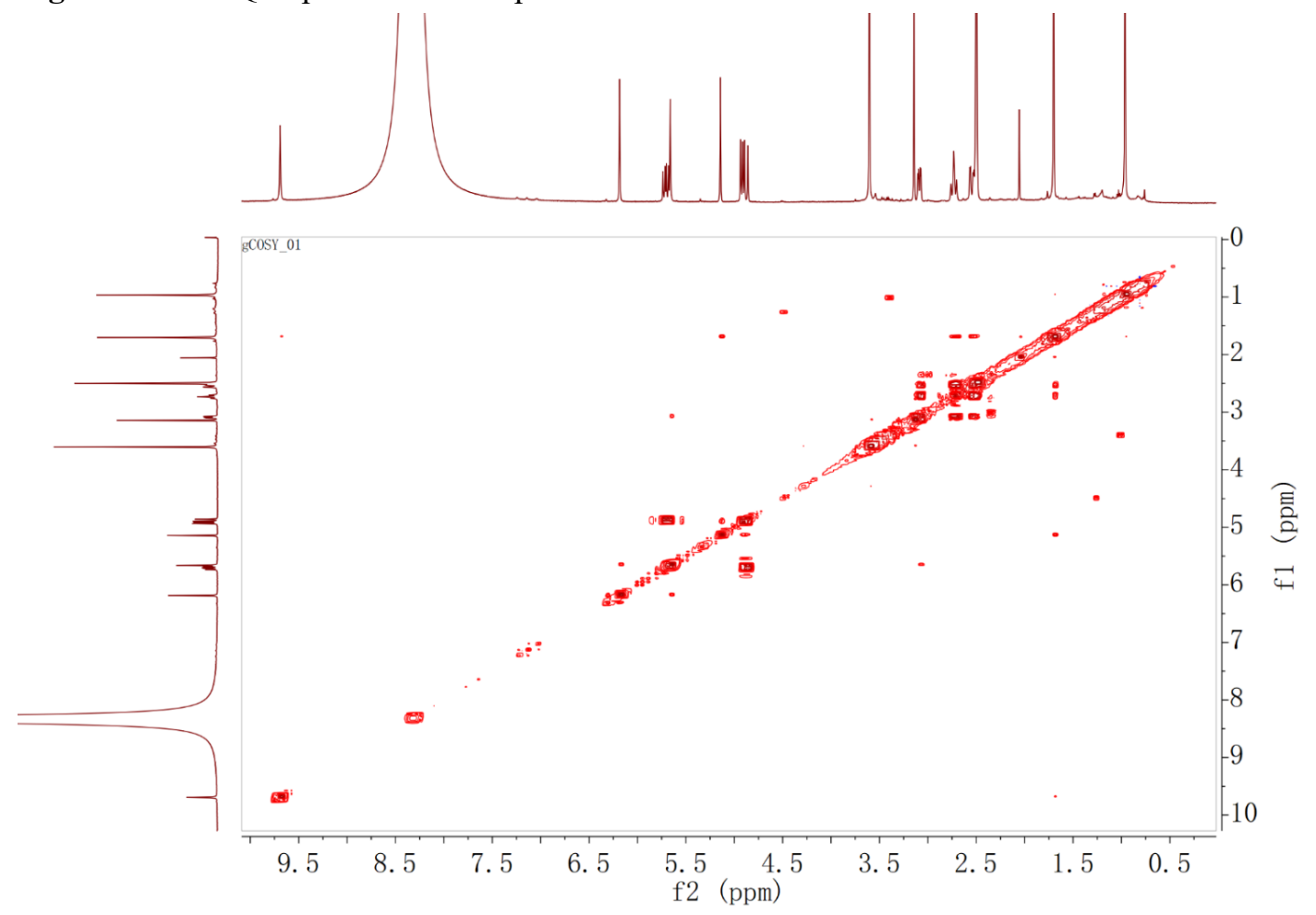

Figure S68. ${ }^{1} \mathrm{H}-{ }^{1} \mathrm{H}-\mathrm{COSY}$ spectrum of compound 8 in DMSO- $d_{6}$. 


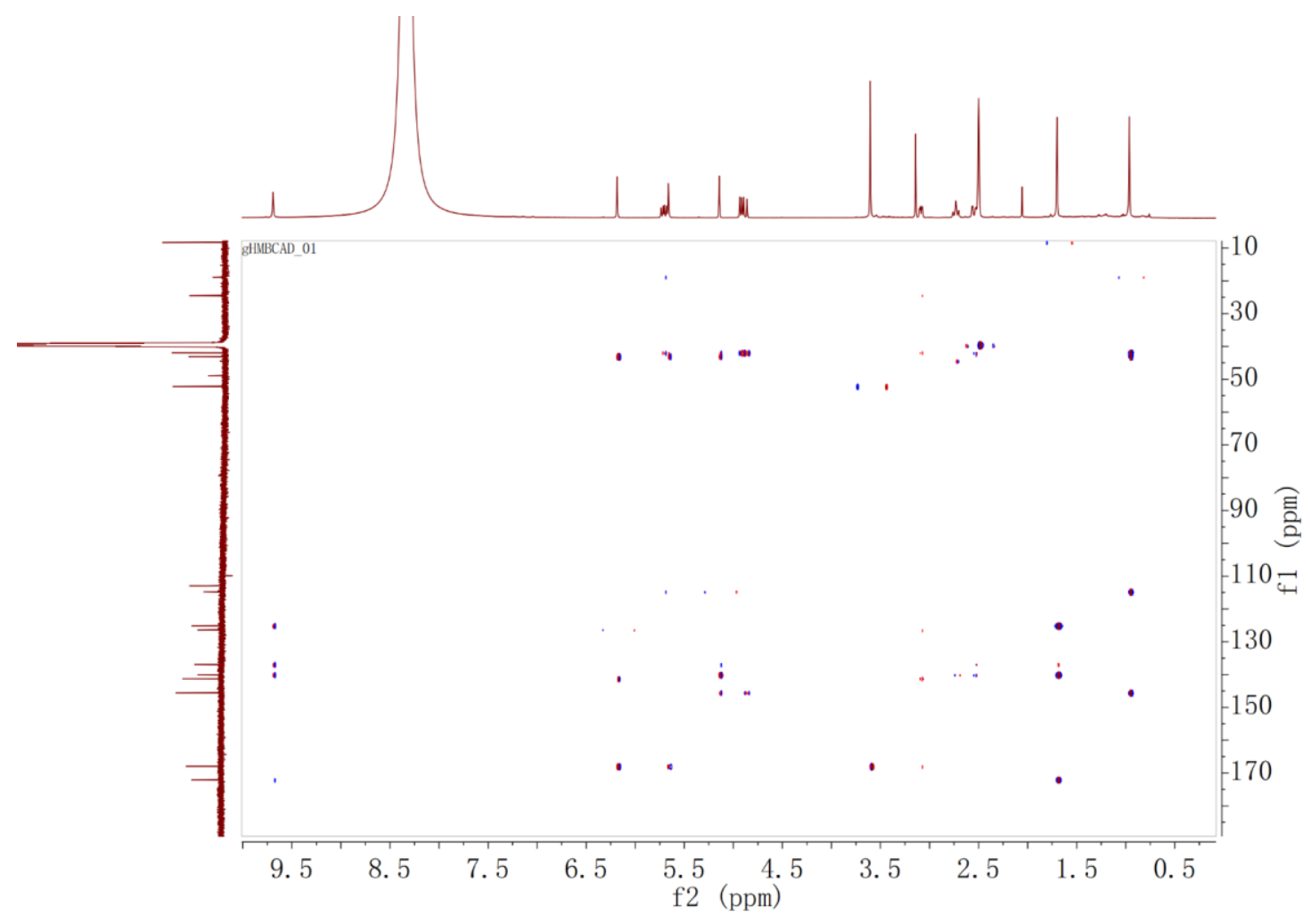

Figure S69. HMBC spectrum of compound 8 in DMSO- $d_{6}$.

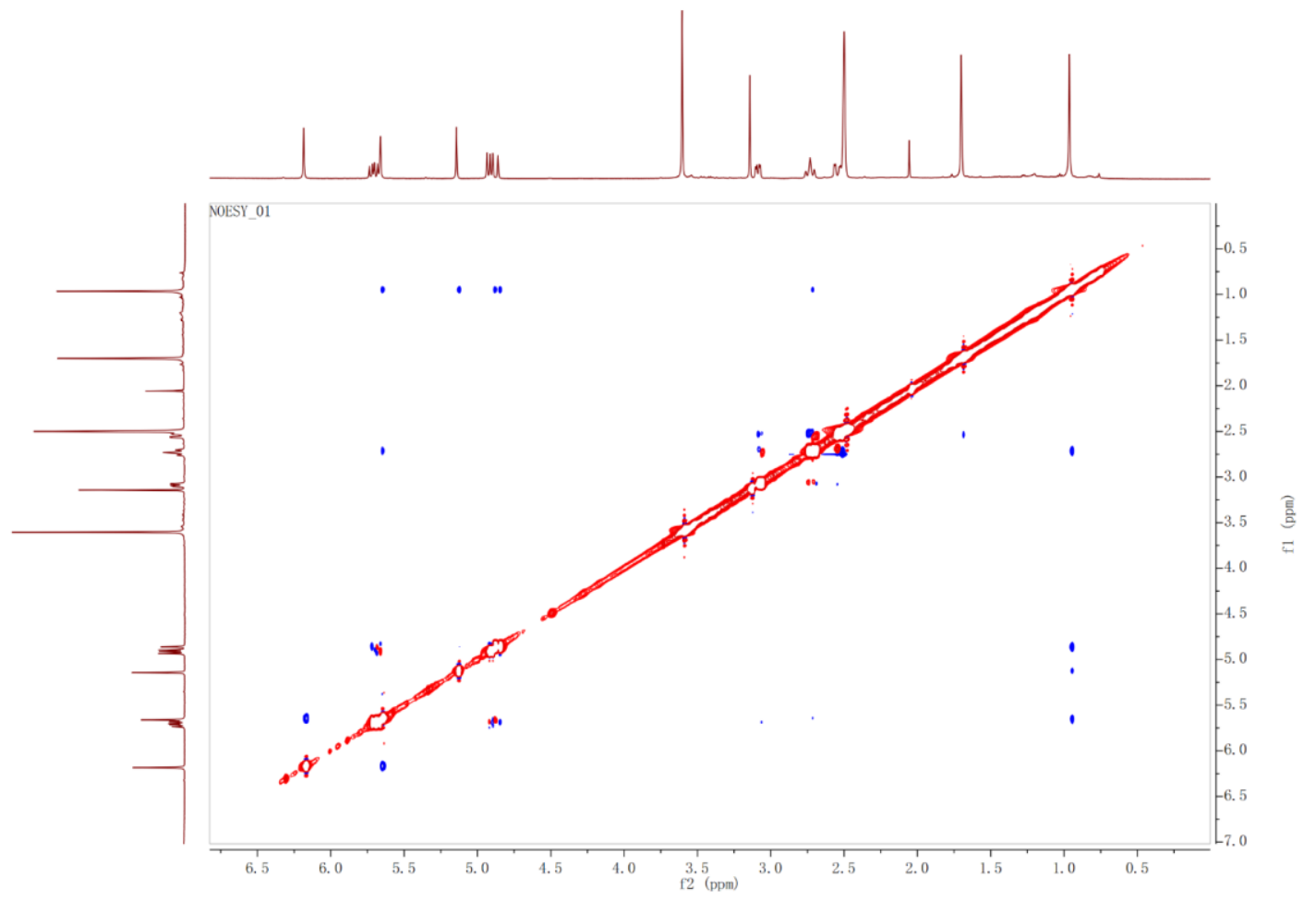

Figure S70. NOESY spectrum of compound 8 in DMSO- $d_{6}$. 


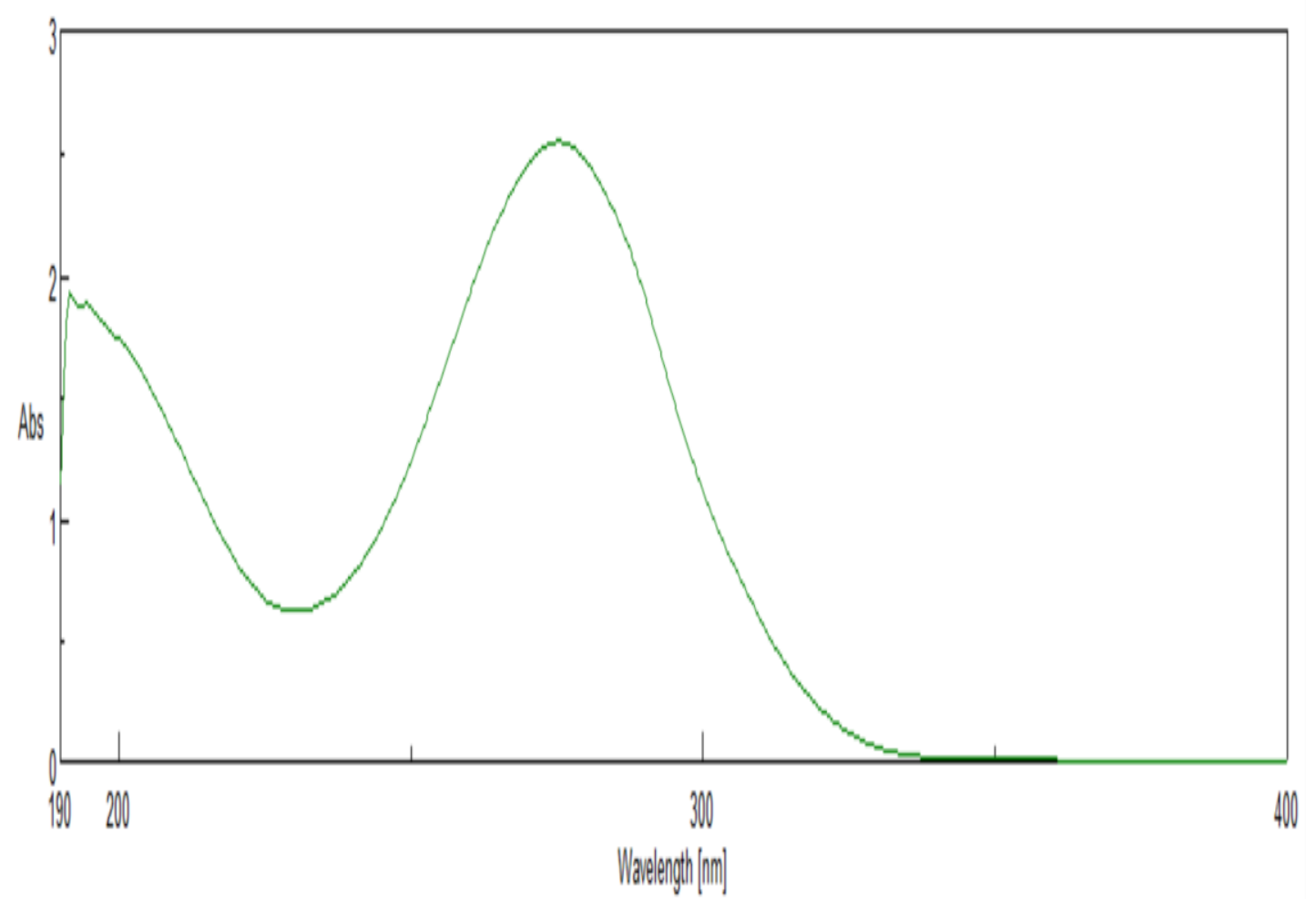

Figure S71. UV spectrum of compound 8.

T: FTMS + p ESI Full ms [100.00-2000.00]

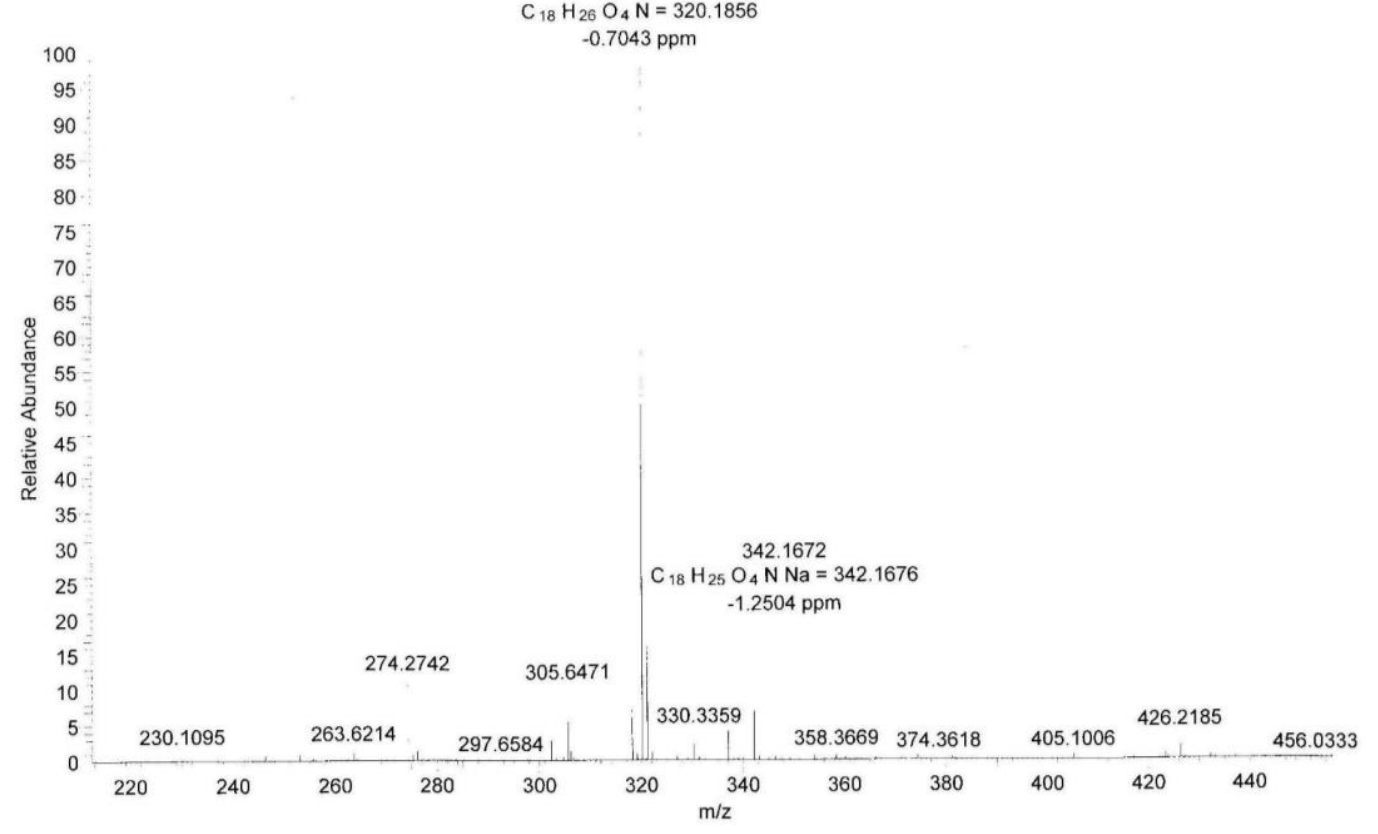

Figure S72. HRESIMS spectrum of compound 9. 


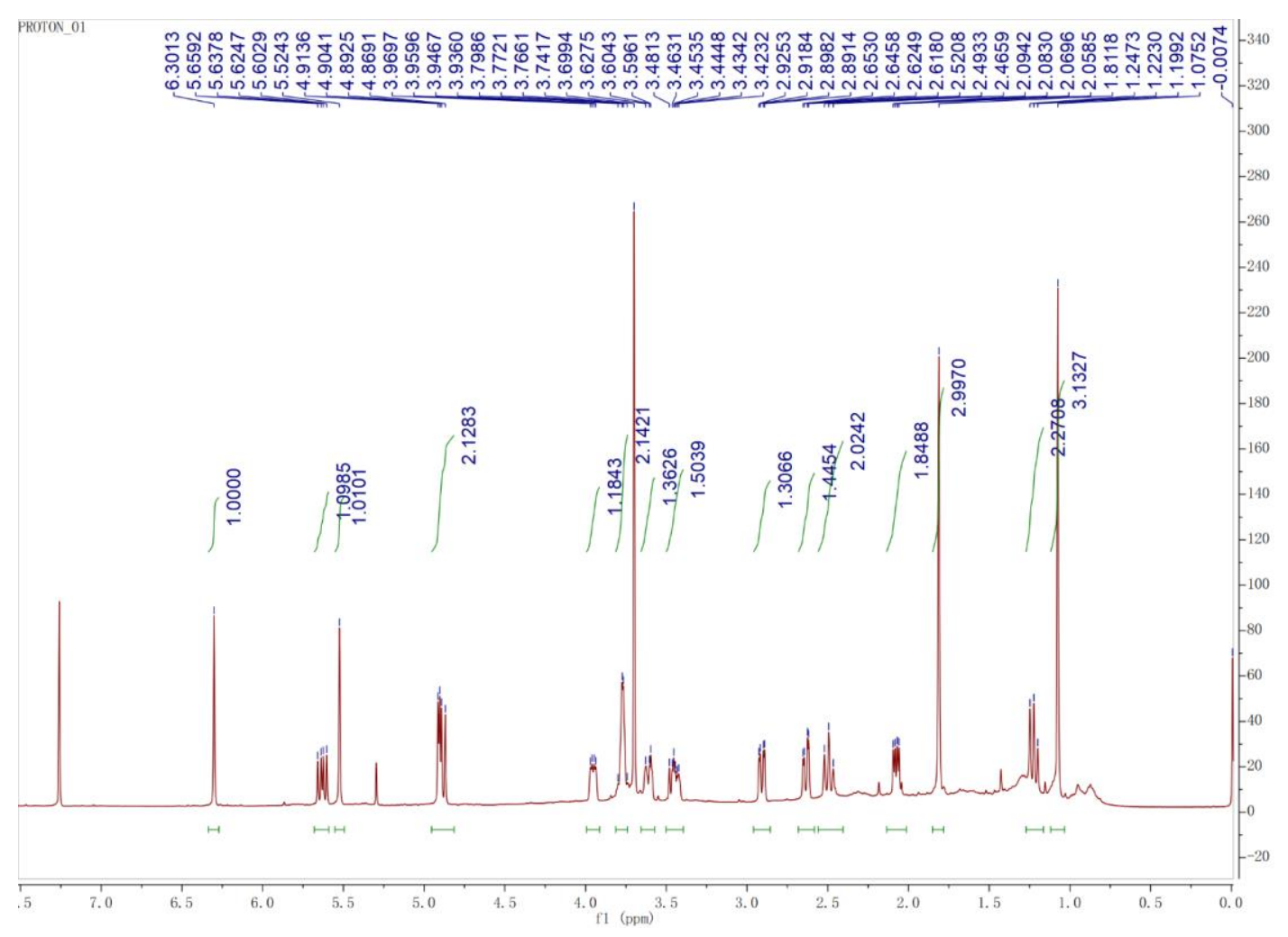

Figure S73. ${ }^{1} \mathrm{H}$ NMR spectrum of compound 9 in $\mathrm{CDCl}_{3}$.

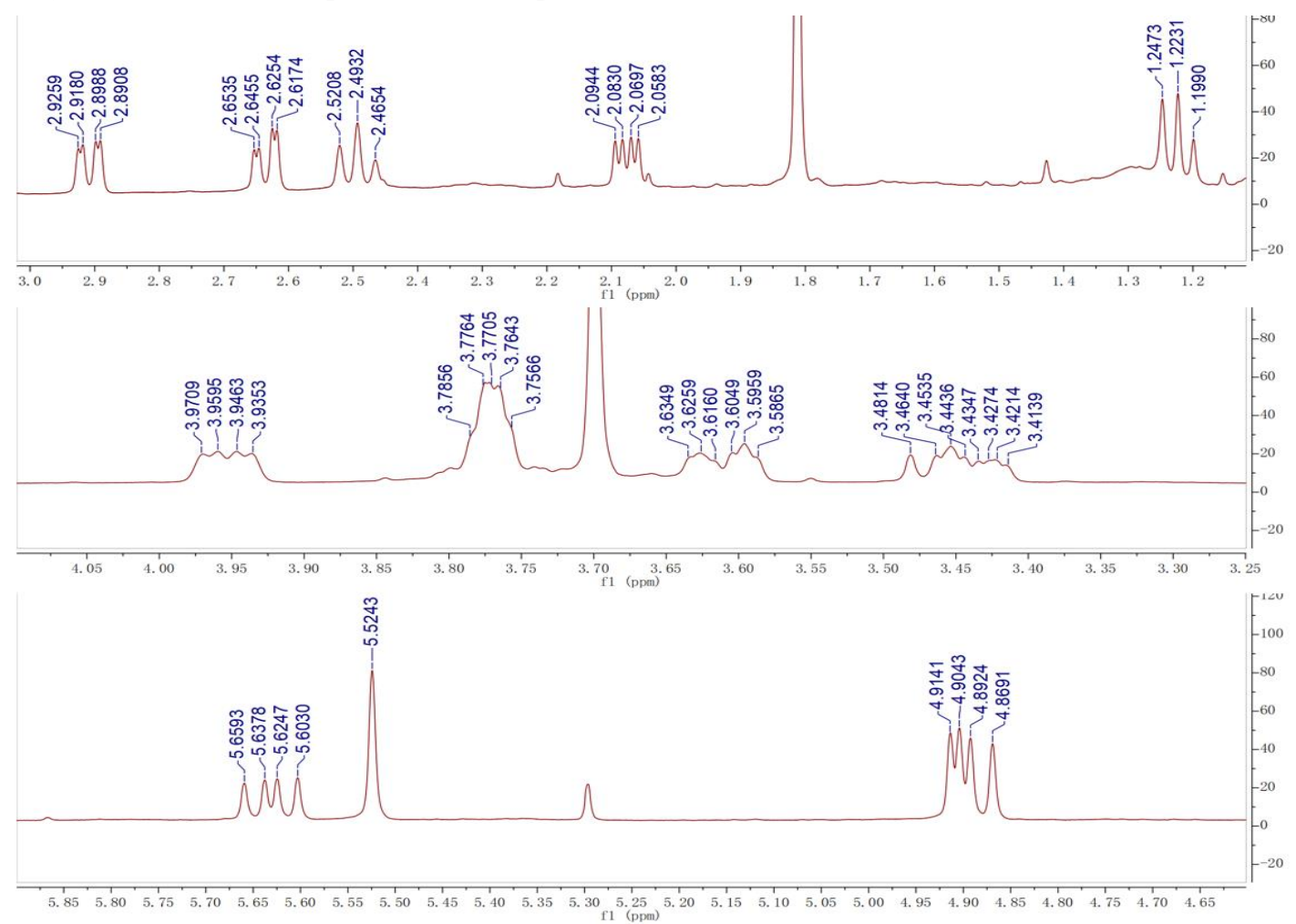

Figure S74. The amplificatory ${ }^{1} \mathrm{H}$ NMR spectrum of compound 9 in $\mathrm{CDCl}_{3}$. 


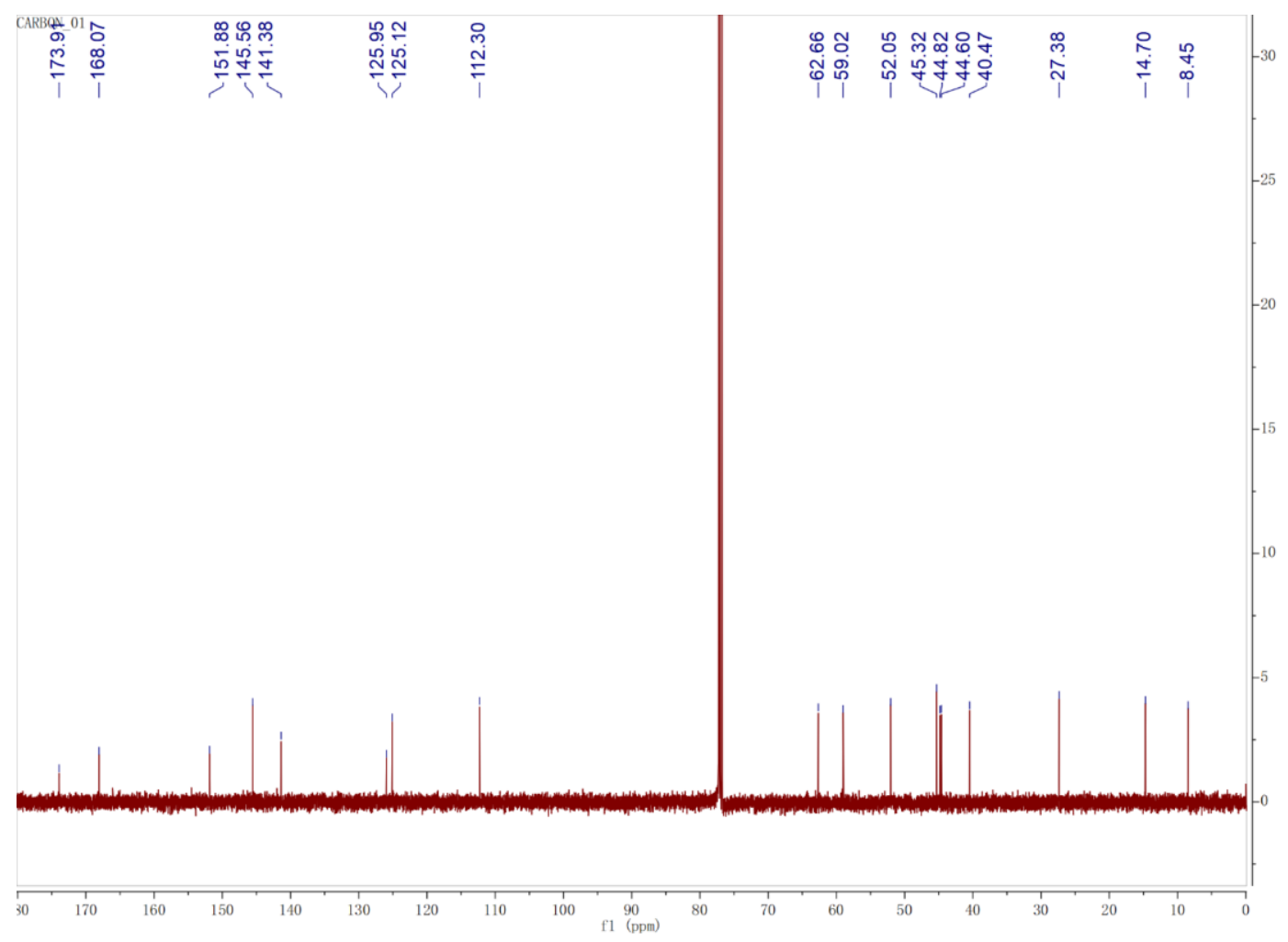

Figure S75. ${ }^{13} \mathrm{C}$ NMR spectrum of compound 9 in $\mathrm{CDCl}_{3}$.

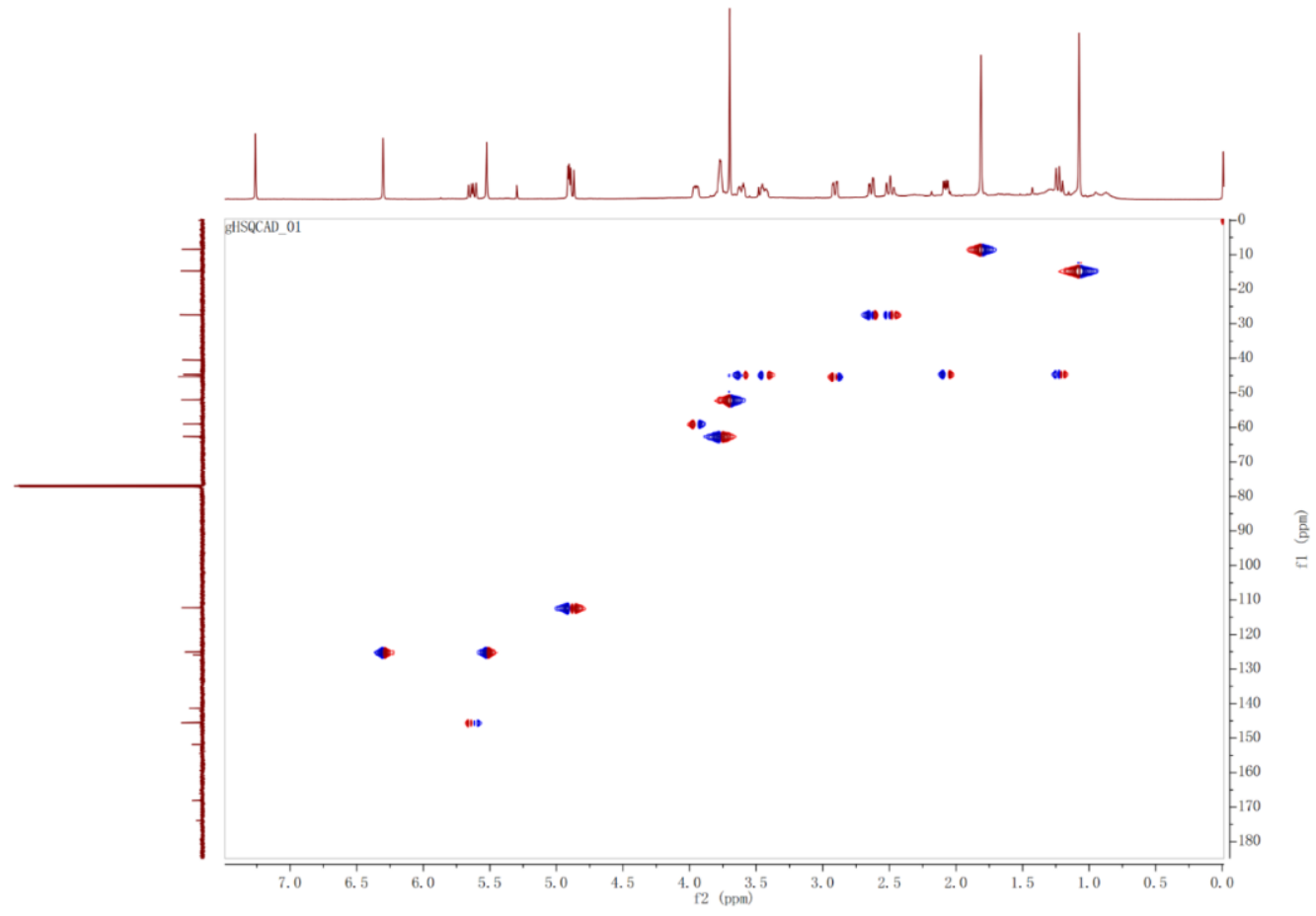

Figure S76. HSQC spectrum of compound 9 in $\mathrm{CDCl}_{3}$. 


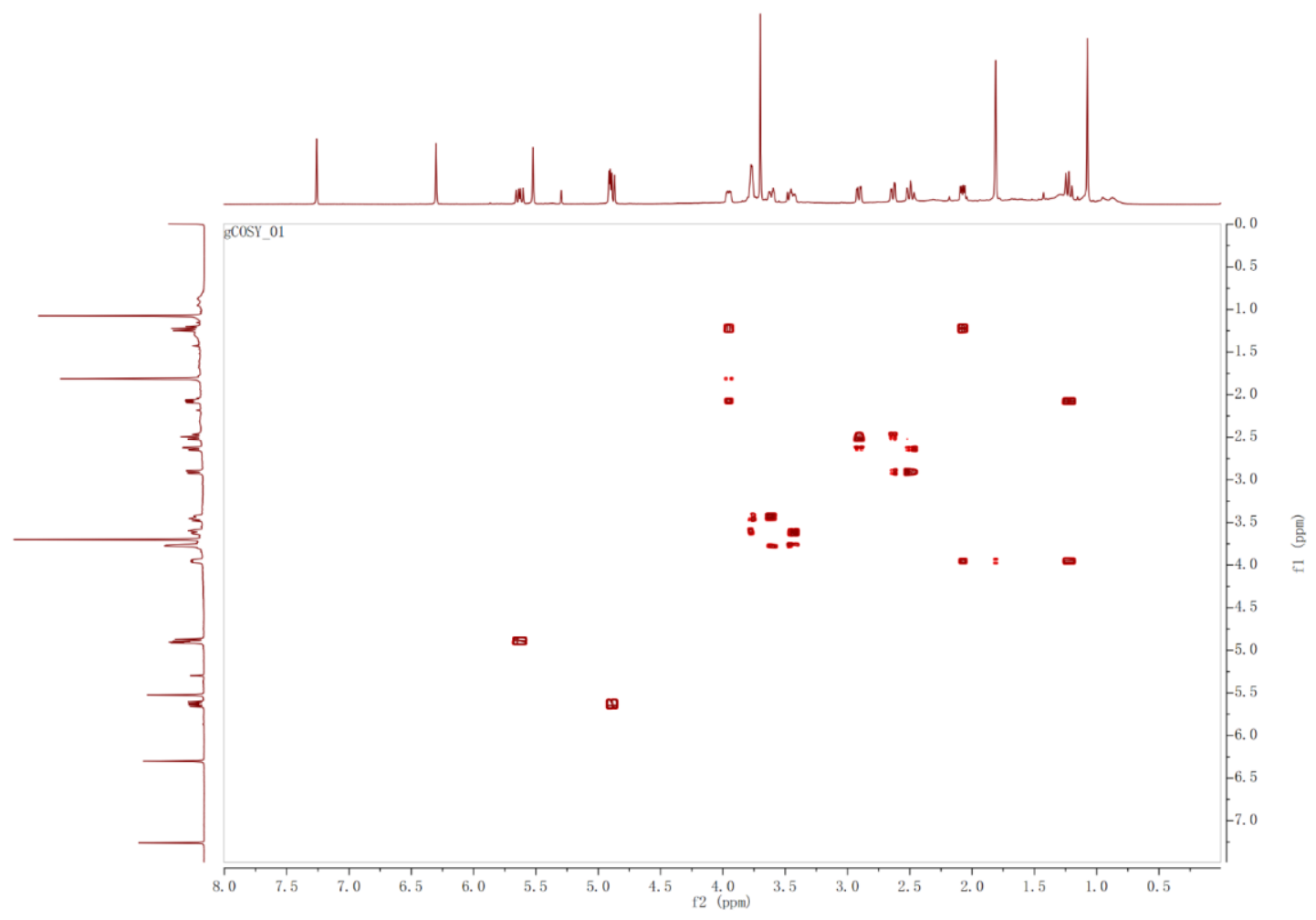

Figure S77. ${ }^{1} \mathrm{H}-{ }^{1} \mathrm{H}-\mathrm{COSY}$ spectrum of compound 9 in $\mathrm{CDCl}_{3}$.

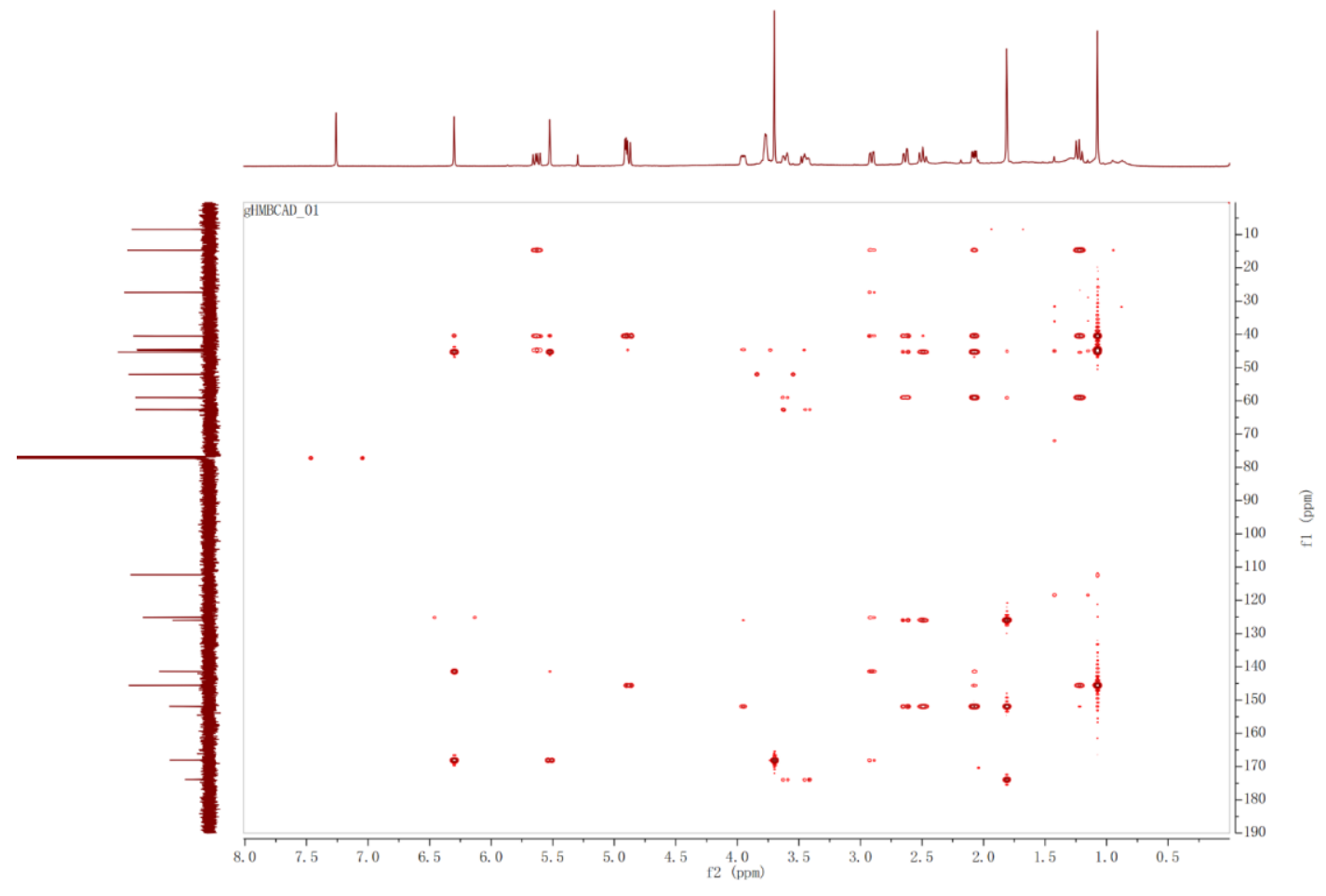

Figure S78. HMBC spectrum of compound 9 in $\mathrm{CDCl}_{3}$. 


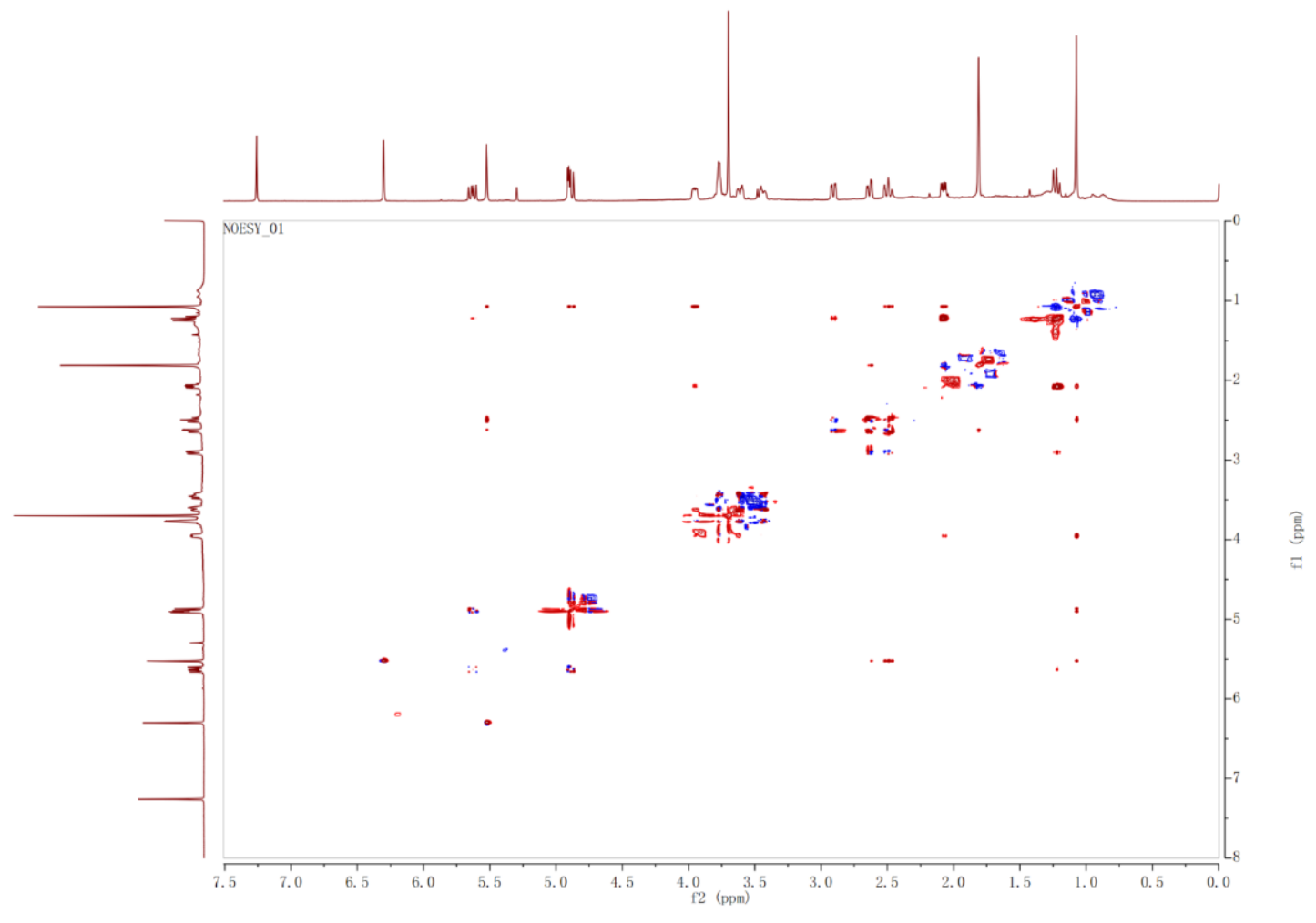

Figure S79. NOESY spectrum of compound 9 in $\mathrm{CDCl}_{3}$.

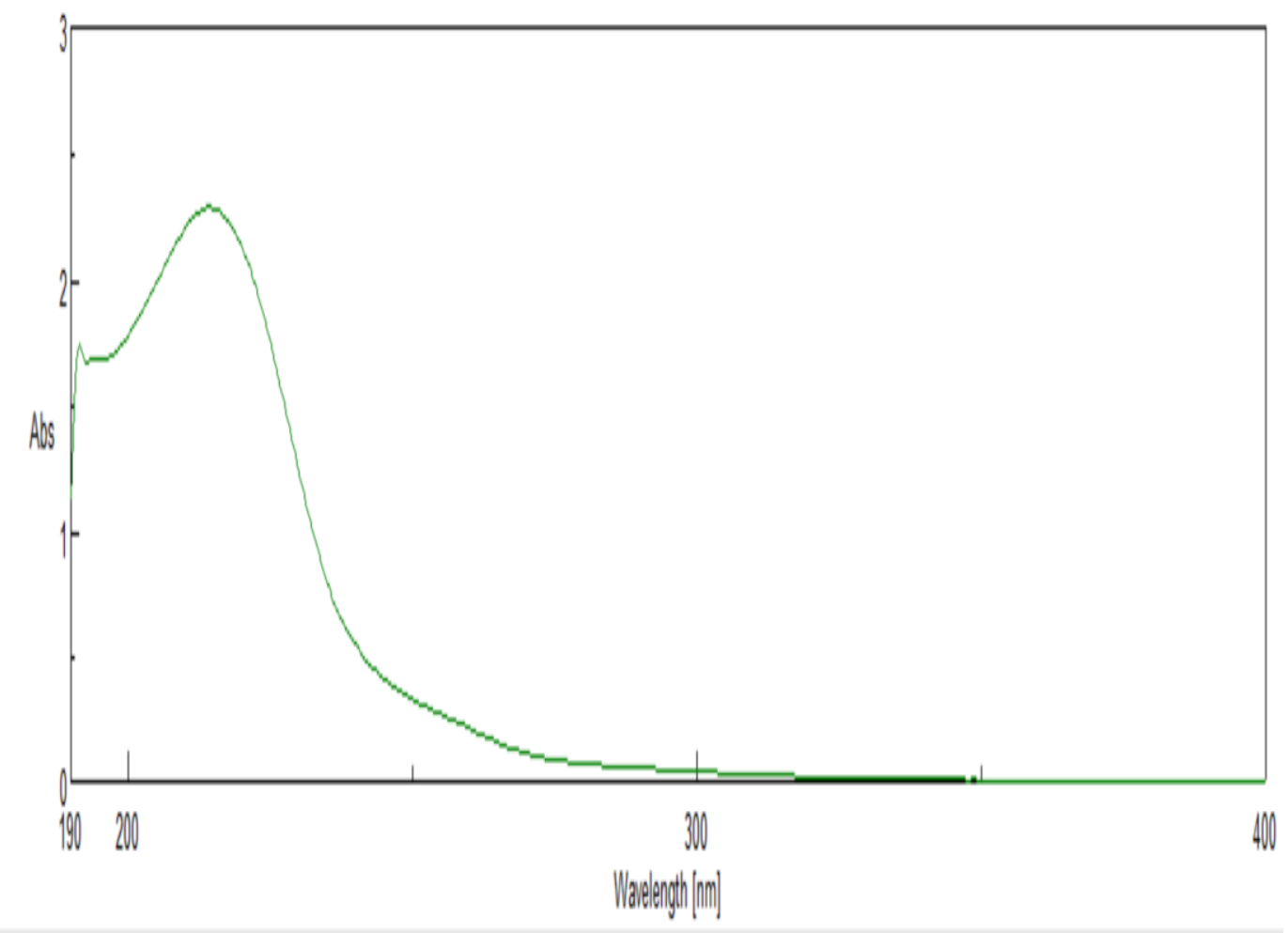

Figure S80. UV spectrum of compound 9. 


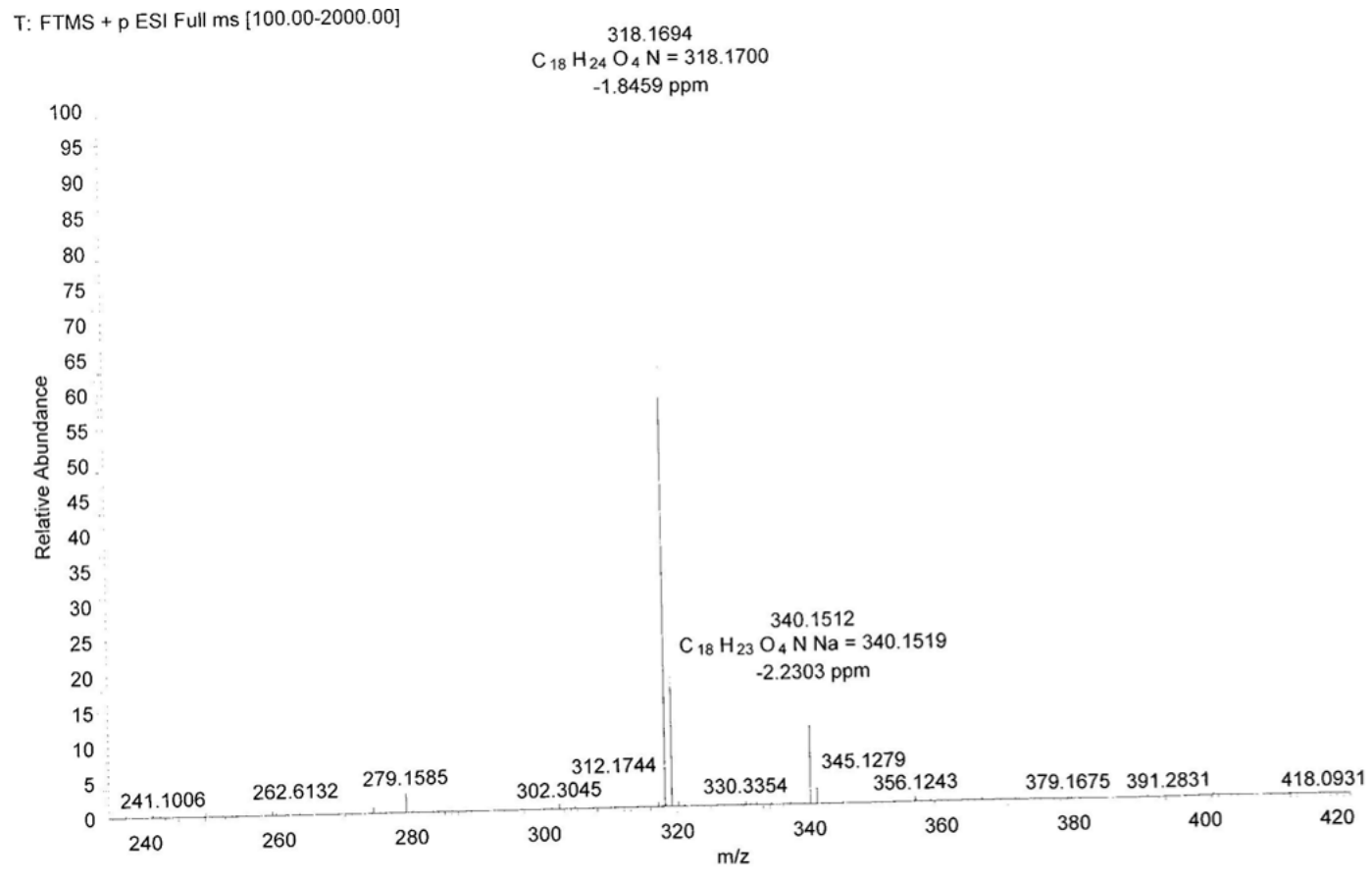

Figure S81. HRESIMS spectrum of compound $\mathbf{1 0}$.

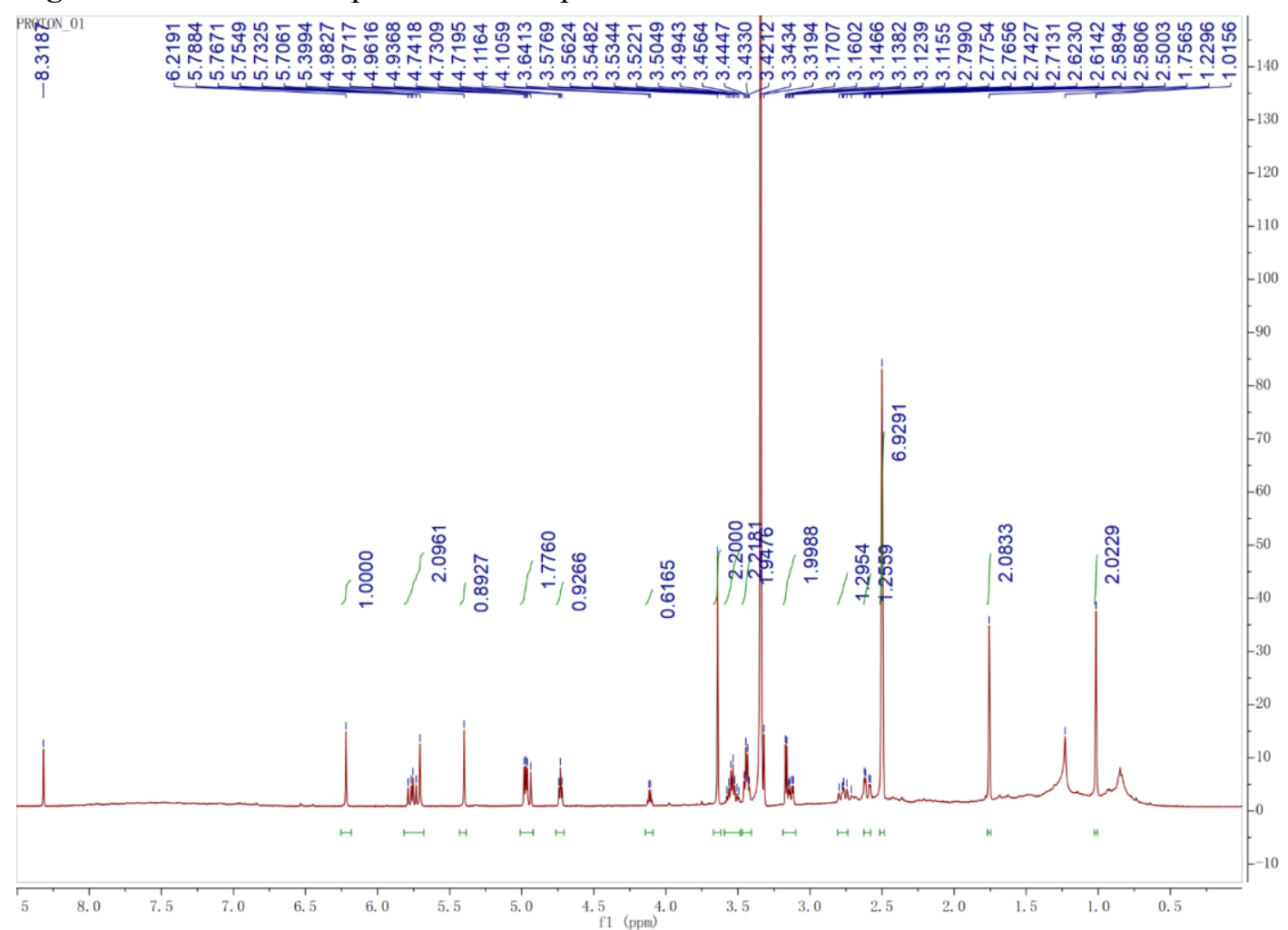

Figure S82. ${ }^{1} \mathrm{H}$ NMR spectrum of compound 10 in DMSO- $d_{6}$. 

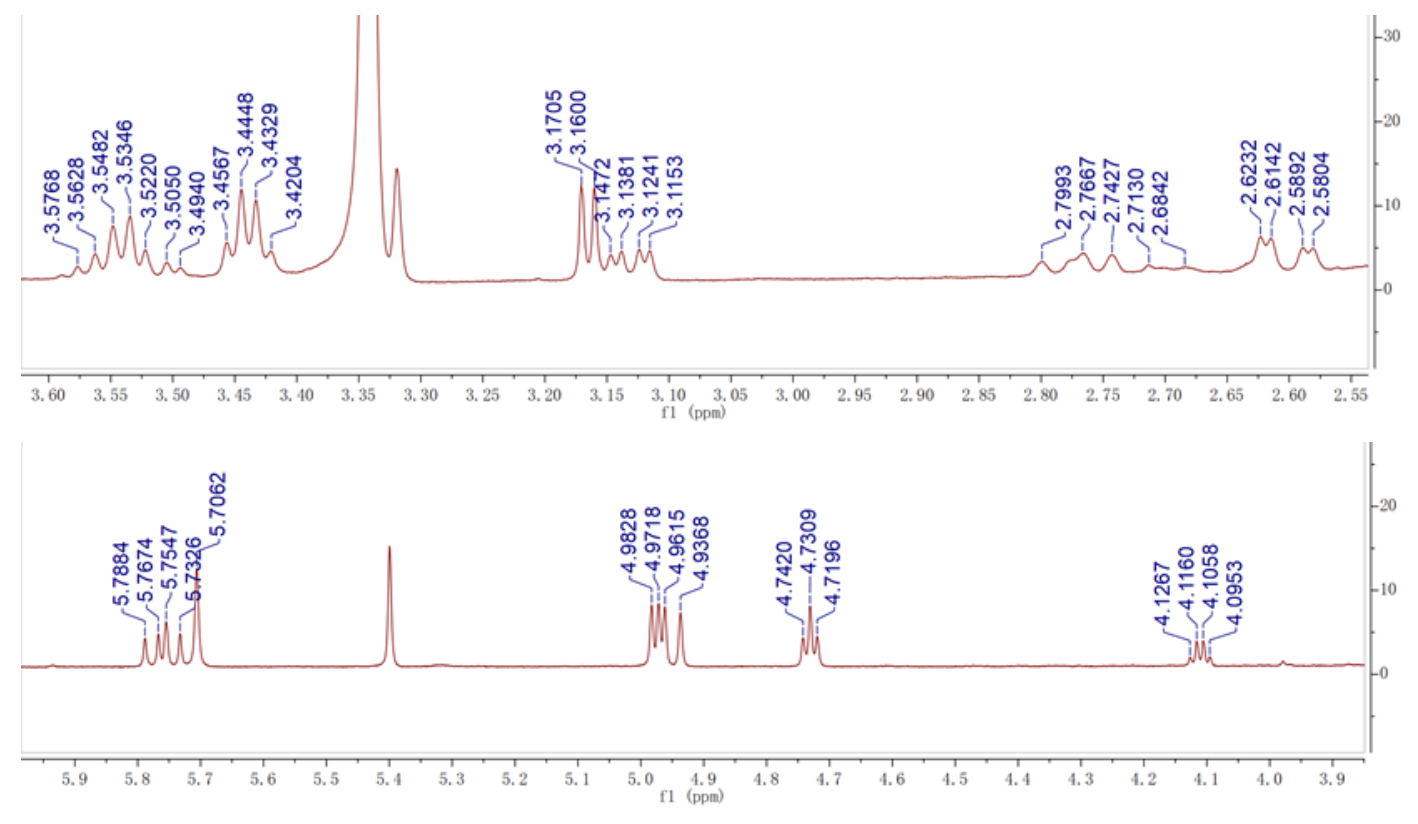

Figure S83. The amplificatory ${ }^{1} \mathrm{H}$ NMR spectrum of compound 10 in DMSO- $d_{6}$.

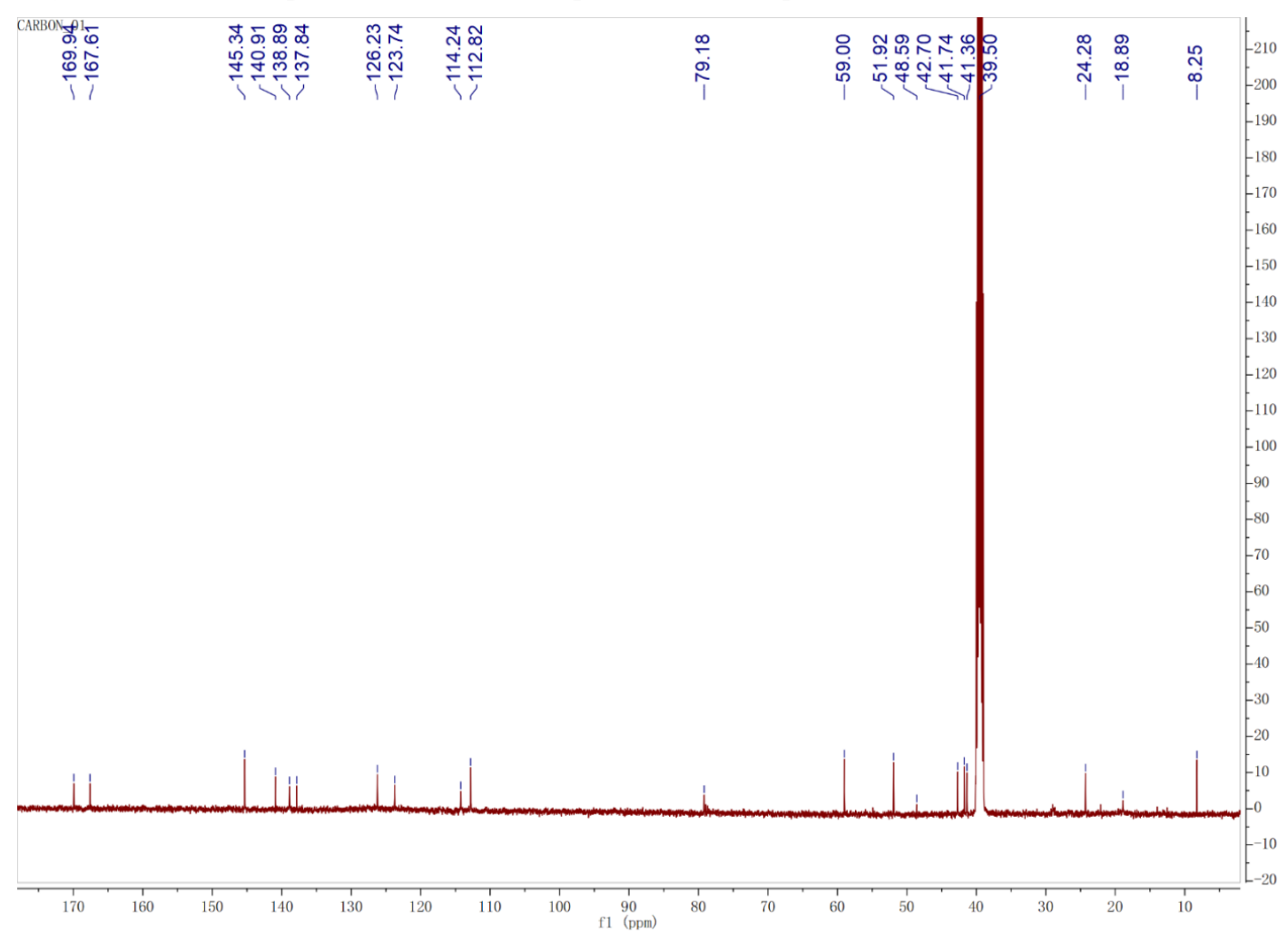

Figure S84. ${ }^{13} \mathrm{C}$ NMR spectrum of compound 10 in DMSO- $d 6$. 


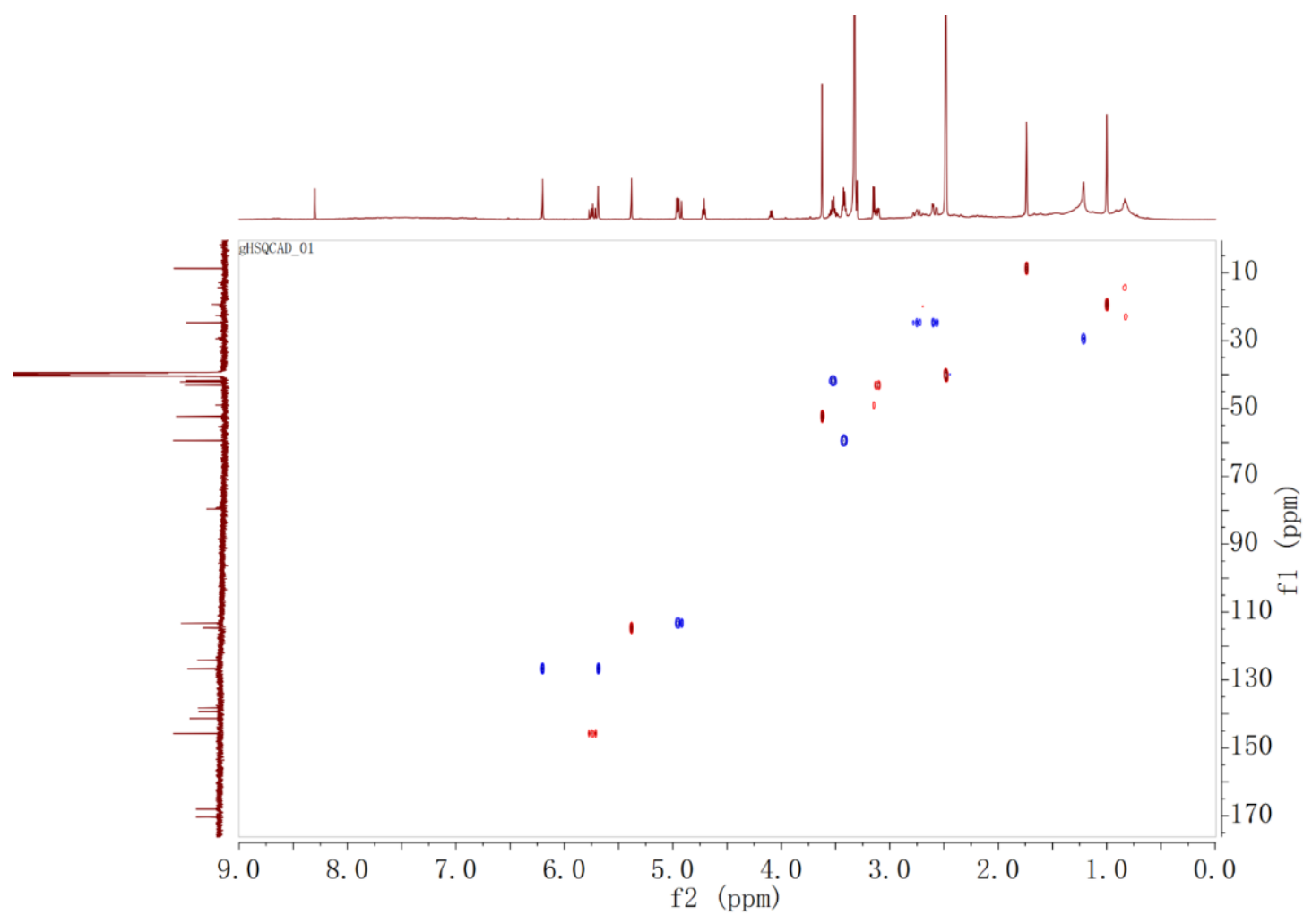

Figure S85. HSQC spectrum of compound 10 in DMSO- $d 6$.

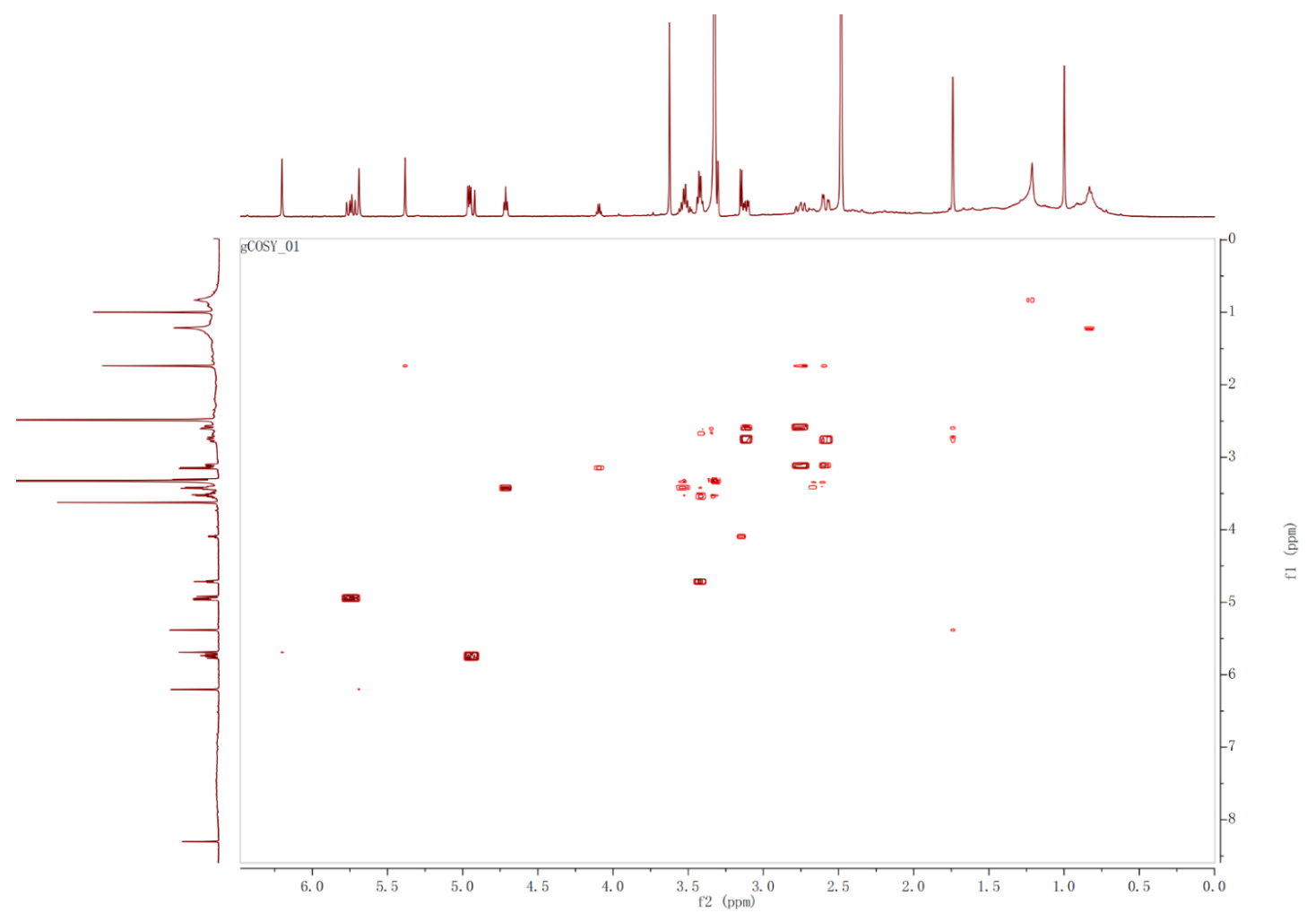

Figure S86. ${ }^{1} \mathrm{H}-{ }^{1} \mathrm{H}-\mathrm{COSY}$ spectrum of compound 10 in DMSO- $d_{6}$. 


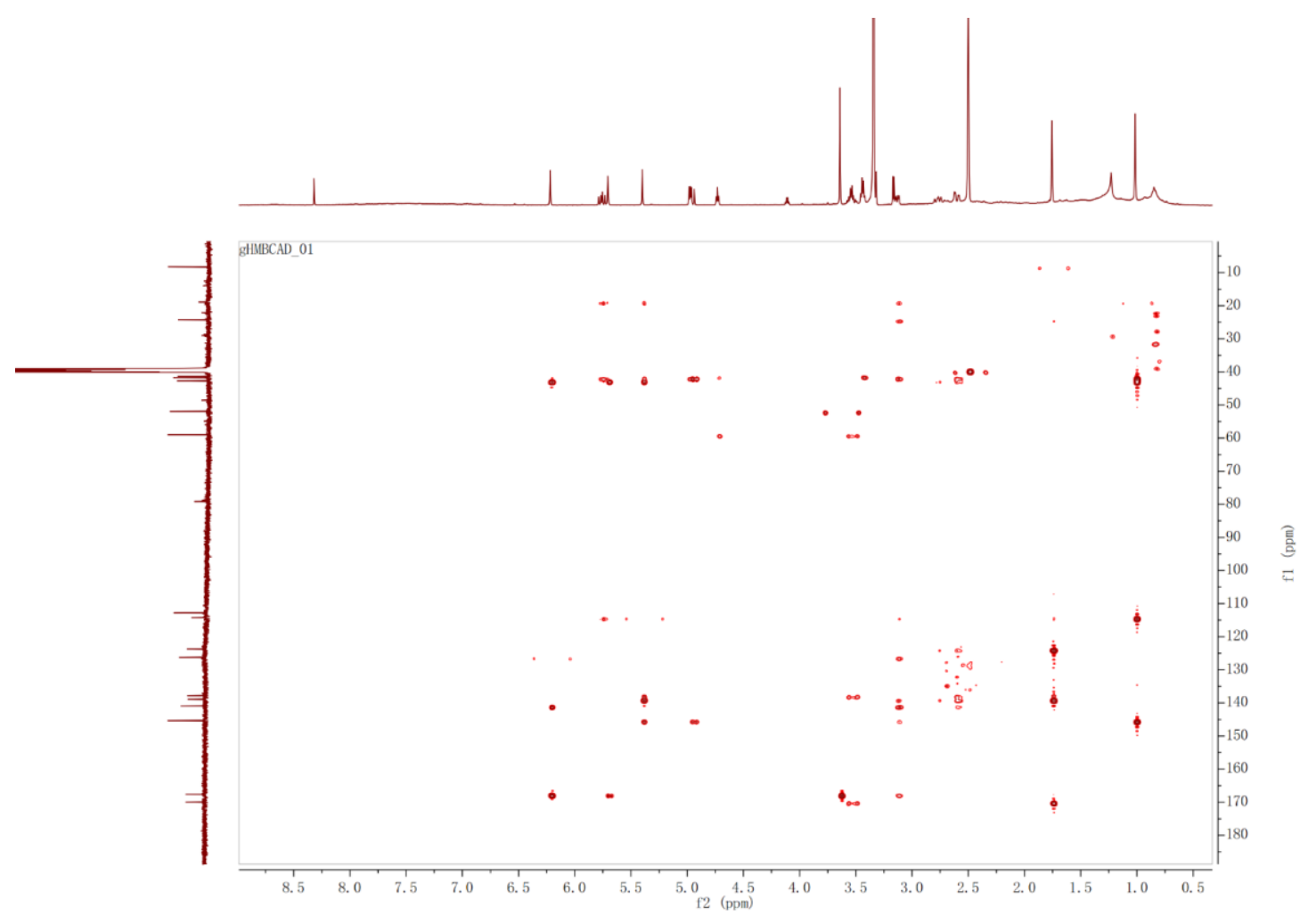

Figure S87. HMBC spectrum of compound 10 in DMSO- $d_{6}$.

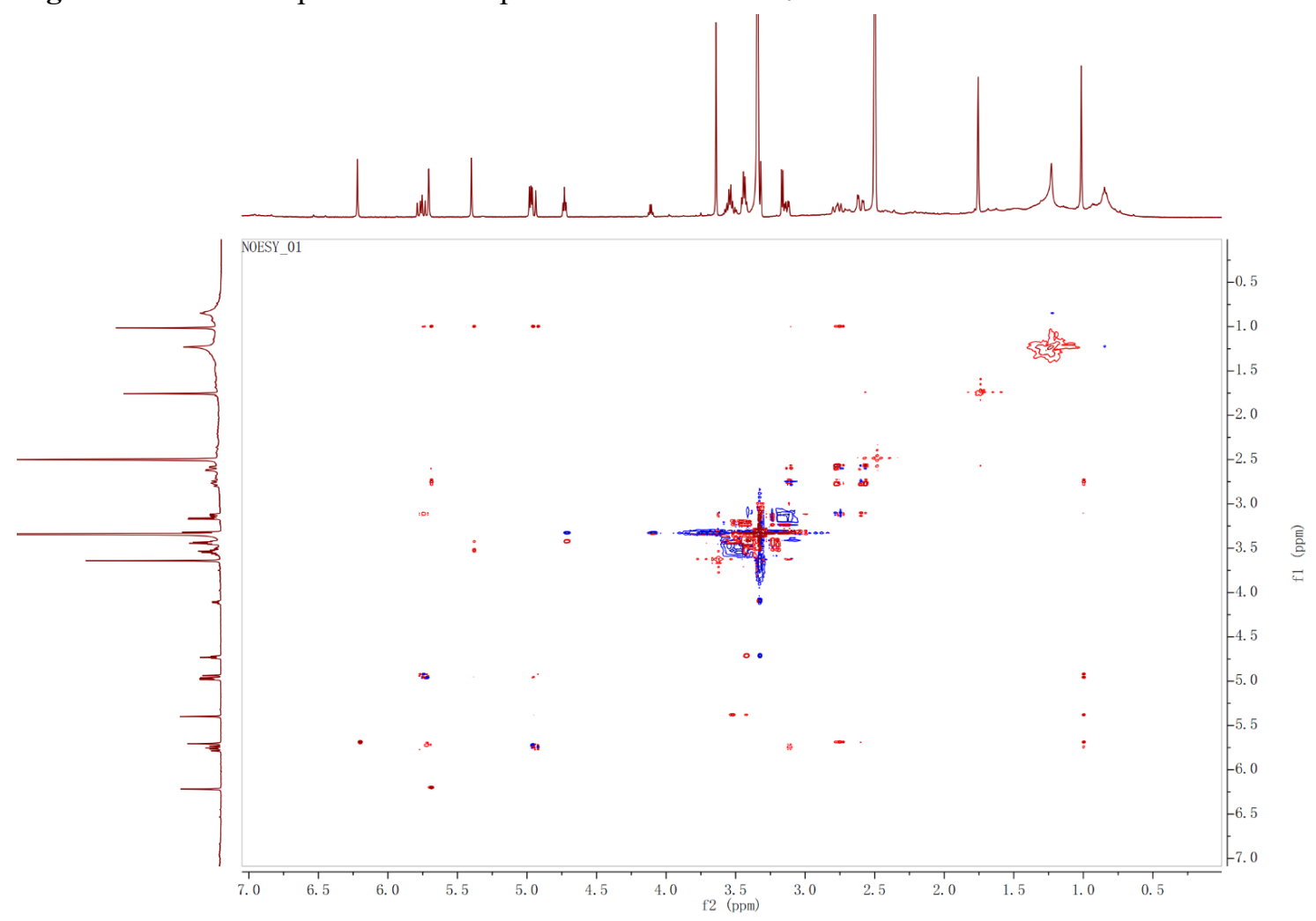

Figure S88. NOESY spectrum of compound 10 in DMSO- $d_{6}$. 


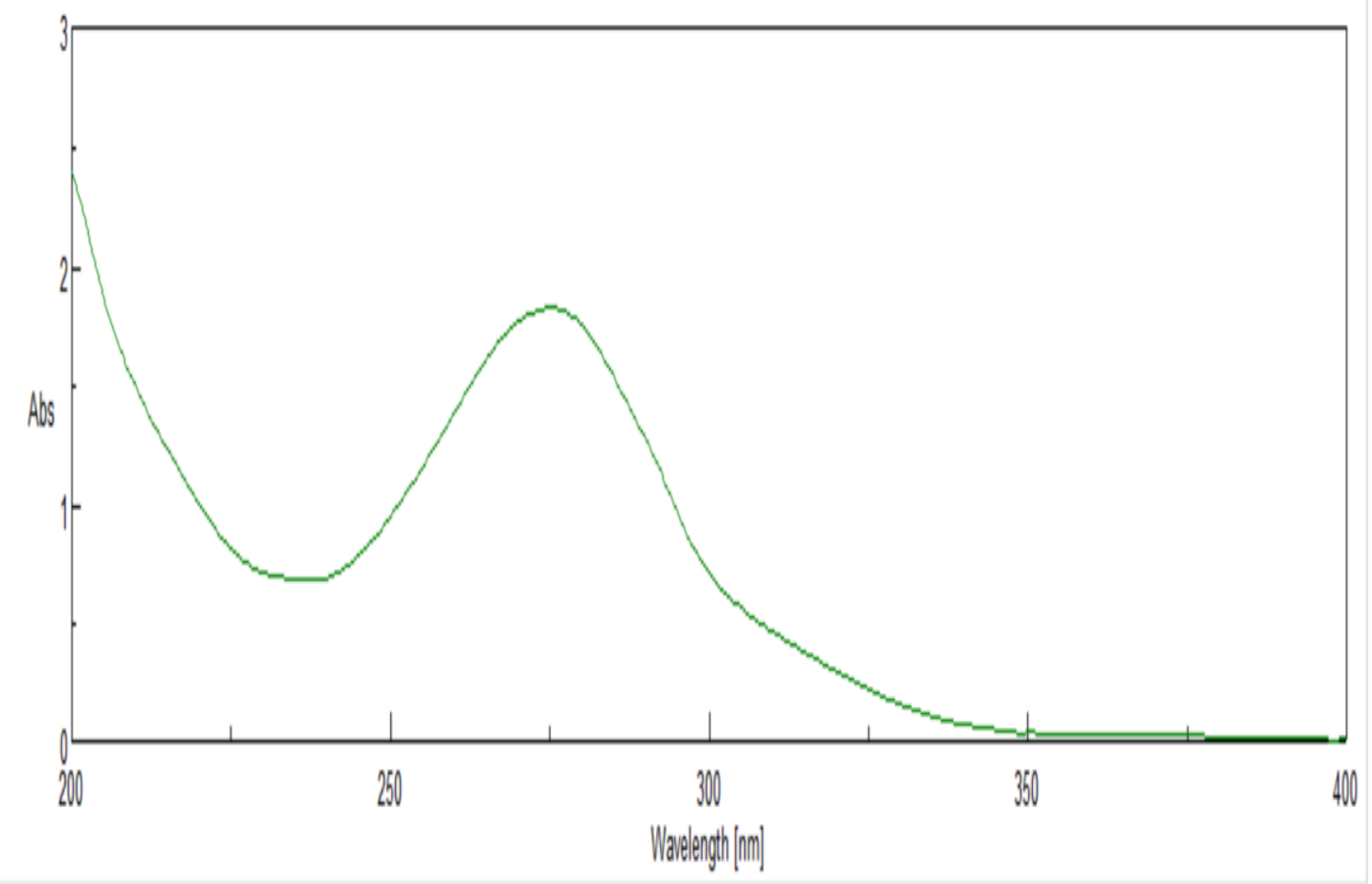

Figure S89. UV spectrum of compound $\mathbf{1 0 .}$

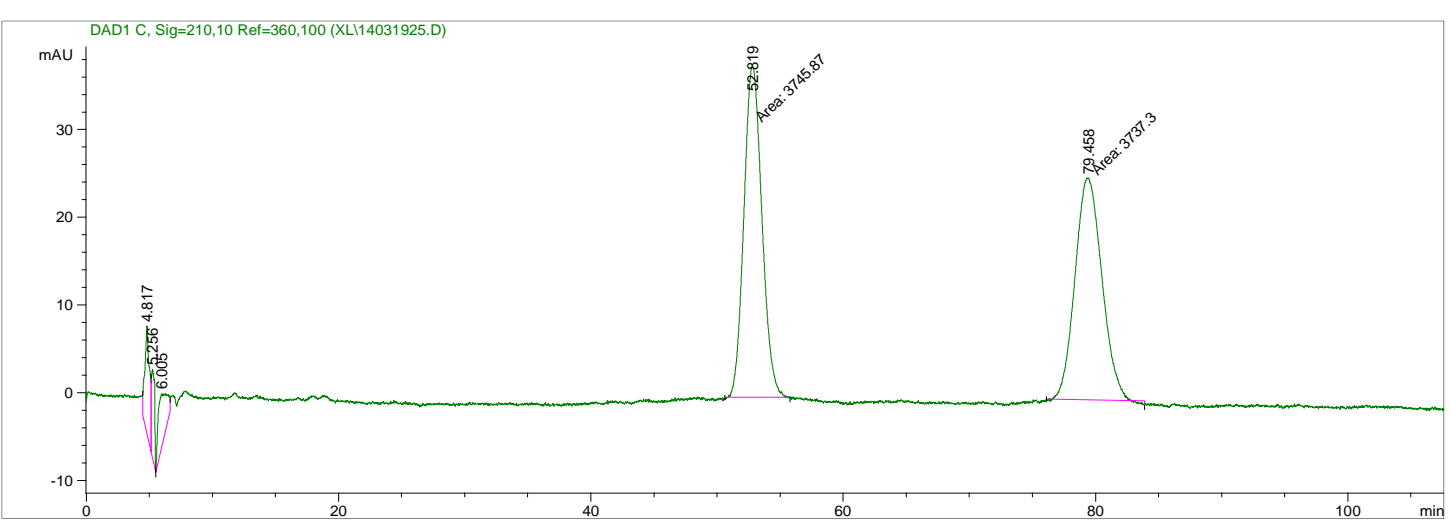

Figure S90. Chiral HPLC chromatogram of compound 7. 